\title{
Who cares? : studying various aspects of involving nurse specialists in primary care for urinary incontinence
}

Citation for published version (APA):

Albers-Heitner, C. P. (2011). Who cares? : studying various aspects of involving nurse specialists in primary care for urinary incontinence. [Doctoral Thesis, Maastricht University]. Datawyse / Universitaire Pers Maastricht. https://doi.org/10.26481/dis.20111201ca

Document status and date:

Published: 01/01/2011

DOI:

10.26481/dis.20111201ca

Document Version:

Publisher's PDF, also known as Version of record

Please check the document version of this publication:

- A submitted manuscript is the version of the article upon submission and before peer-review. There can be important differences between the submitted version and the official published version of record.

People interested in the research are advised to contact the author for the final version of the publication, or visit the DOI to the publisher's website.

- The final author version and the galley proof are versions of the publication after peer review.

- The final published version features the final layout of the paper including the volume, issue and page numbers.

Link to publication

\footnotetext{
General rights rights.

- You may freely distribute the URL identifying the publication in the public portal. please follow below link for the End User Agreement:

www.umlib.nl/taverne-license

Take down policy

If you believe that this document breaches copyright please contact us at:

repository@maastrichtuniversity.nl

providing details and we will investigate your claim.
}

Copyright and moral rights for the publications made accessible in the public portal are retained by the authors and/or other copyright owners and it is a condition of accessing publications that users recognise and abide by the legal requirements associated with these

- Users may download and print one copy of any publication from the public portal for the purpose of private study or research.

- You may not further distribute the material or use it for any profit-making activity or commercial gain

If the publication is distributed under the terms of Article $25 \mathrm{fa}$ of the Dutch Copyright Act, indicated by the "Taverne" license above, 


\section{WHO CARES? \\ Studying various aspects of involving nurse specialists in primary care for urinary incontinence}


The research presented in this thesis was conducted at the Maastricht University Medical Centre ${ }^{+}$, Department of Integrated Healthcare and the School for Public Health and Primary Care: CAPHRI. CAPHRI participates in the Netherlands School of Primary Care Research CaRe, which has been acknowledged since 1995 by the Royal Netherlands Academy of Arts and Sciences (KNAW). CAPHRI was classified as 'excellent' by the external evaluation committee of leading international experts that reviewed CAPHRI in December 2010.

Financial support for printing this thesis was generously provided by

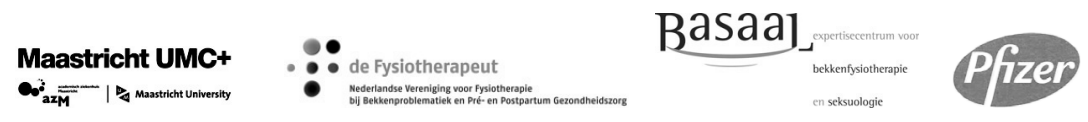

ISBN 9789461590909

(C) Pytha Albers-Heitner, 2011

No part of this thesis may be reproduced, stored in retrieval system or transmitted in any forms by any means, electronic or mechanical, including photocopying, recording, or any information storage and retrieval system without written permission of the copyright owner.

Cover design: $\mathbf{x a}$ xanderalbers.nl

Production: Datawyse | Universitaire Pers Maastricht 


\title{
WHO CARES? \\ Studying various aspects of involving nurse specialists in primary care for urinary incontinence
}

\author{
PROEFSCHRIFT
}
ter verkrijging van de graad van doctor aan de Universiteit Maastricht, op gezag van de Rector Magnificus, Prof. mr. G.P.M.F. Mols volgens het besluit van het College van Decanen, in het openbaar te verdedigen op donderdag 1 december 2011 om 16.00 uur

door

Catharina Pythia Albers-Heitner

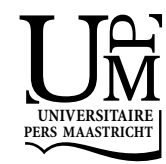




\section{Promotores}

Prof. dr. J.L. Severens (Erasmus Universiteit Rotterdam)

Prof. dr. A.L.M. Lagro-Janssen (Universitair Medisch Centrum St Radboud)

\section{Copromotores}

Dr. L.C.M. Berghmans

Dr. R.A.G. Winkens

\section{Beoordelingscommissie}

Prof. dr. Ph.E.V.A. Van Kerrebroeck (voorzitter)

Dr. M.G.W. Dijkgraaf (Academisch Medisch Centrum Amsterdam)

Prof. dr. J.P.H. Hamers

Prof. dr. J.F.M. Metsemakers

Prof. dr. M.W.G. Nijhuis-Van der Sanden (Universitair Medisch Centrum St Radboud) 
Alleen passie maakt het leven de moeite waard Sándor Márai (Gloed) 1942 
Uit de praktijk:

Een ervaring rijker en een (groot) probleem minder.

Enkele jaren geleden werd het veelvuldig plassen en het immer ongelegen komende urineverlies, langzamerhand een obsessie. De constante paniek die bij mij ontstond als er geen plasgelegenheid in de buurt was, speelde me danig parten. Het bewust wegblijven bij activiteiten werd een steeds vaker voorkomend gebeuren. De diverse (soms vervelende) onderzoeken op de afdeling urologie, gaven geen duidelijk medisch of lichamelijk probleem. In dat opzicht dus een hele geruststelling, maar het probleem was daarmee niet opgelost. Een voorgeschreven bekkenbodemtherapie en een ondersteunende medicatie gaven wel wat verbetering maar 24 á 25 maal per etmaal naar het toilet, verstoorde mijn dagelijkse werkzaamheden, maar ook zeker mijn nachtrust.

Medio december 2005 werd mij via de huisarts gevraagd, deel te nemen de PromoCon studie. Achteraf ben ik bijzonder blij, dat ik toen door loting aan de in incontinentie gespecialiseerd verpleegkundige werd toevertrouwd.

Het traject via huisarts en medisch specialist had ik al grotendeels doorlopen en had voor mij niet het gewenste resultaat opgeleverd. Dus ik was erg benieuwd wat een gespecialiseerd verpleegkundige daar nog aan kon toevoegen. Haar andere aanpak en het nadrukkelijk kijken naar de manier van leven, drinken, eet- en plasgewoontes zorgde uiteindelijk voor de ommekeer. Weliswaar ging het zeker niet zonder slag of stoot, want ik had een aantal gewoontes die je na 45 jaar niet makkelijk ombuigt ...

Maar juist de vasthoudendheid van mijn verpleegkundige gaf mij uiteindelijk de positieve stimulans om vol te houden en binnen één jaar een maximaal resultaat te halen.

Een resultaat dat er mag zijn: van circa 12 á 13 bekers koffie per dag terug naar 5 á 6 kopjes, de juiste plastechnieken aangeleerd, het toiletbezoek terug gebracht naar 6 á 7 keer per etmaal en geen urineverlies meer. En zeker niet onbelangrijk in dit gebeuren: de hoeveelheid drinken nagenoeg gelijk gebleven ten opzichte van voor die tijd. Géén panische angsten meer en een betere nachtrust, waardoor ik overdag energieker ben. Dus al met al "voor mij een groot probleem minder en voor het onderzoeksteam een positieve ervaring rijker." Hieruit blijkt dat het zeker anders kan of liever gezegd zou moeten. Bedankt voor dit geweldige resultaat!

De heer J.S. uit G. 


\section{Content}

$\begin{array}{ll}\text { Abbreviations } & 9\end{array}$

$\begin{array}{lll}\text { Chapter } 1 & \text { General Introduction } & 11\end{array}$

Part I Usual care for patients with urinary incontinence

Chapter $2 \quad$ Adherence to professional guidelines for patients with 31

urinary incontinence by general practitioners: A cross-

sectional study

Journal of Evaluation in Clinical Practice. 2008;14(5):807-

811.

Chapter 3 How do patients with urinary incontinence perceive care

given by their general practitioner? A cross-sectional study

The International Journal of Clinical Practice.

2008;62(3):508-515.

Part II Involvement of nurse specialists in primary care for urinary incontinence

Chapter 4 Study protocol. The effects of involving a nurse

practitioner in primary care for adult patients with urinary incontinence: The PromoCon study (Promoting

Continence)

BMC Health Services Research. 2008(8):84.

Chapter 5 Effectiveness of involving a nurse specialist for patients

with urinary incontinence in primary care: Results of a pragmatic multicentre randomised controlled trial The International Journal of Clinical Practice. 2011;65(6):705-712.

Chapter 6 Cost-effectiveness of involving nurse specialists for adult patients with urinary incontinence in primary care compared to care-as-usual: An economic evaluation alongside a pragmatic randomised controlled trial Neurourology and Urodynamics. (Accepted 08-07-2011). 
Chapter 7 Experiences and attitudes of nurse specialists in primary care regarding their role in care for patients with urinary incontinence

Scandinavian Journal of Caring Sciences. 2011;25(2):303310.

Chapter 8 Consumer satisfaction among patients and their general practitioners about involving nurse specialists in primary care for patients with urinary incontinence

Submitted.

Chapter 9 General Discussion

Summary

Samenvatting

Co-authors \& affiliations

Dankwoord 189

Curriculum Vitae

197

Publications 


\section{Abbreviations}

\begin{tabular}{|c|c|}
\hline ANOVA & Analysis of Variance \\
\hline AUC & Area-under-the-curve \\
\hline $\mathrm{BCl}$ & Bootstrap Confidence Interval \\
\hline $\mathrm{BMI}$ & $\begin{array}{l}\text { Body Mass Index, calculated as weight in kilograms divided by height in } \\
\text { meters squared }\end{array}$ \\
\hline CEA & Cost-Effectiveness Analysis \\
\hline CEAC & Cost-Effectiveness Acceptability Curve \\
\hline $\mathrm{Cl}$ & Confidence Interval \\
\hline CRF & Case Record Form \\
\hline $\mathrm{CQ}$ & Cost Questionnaire \\
\hline EVPI & Expected Value of Perfect Information \\
\hline$E Q-5 D$ & $\begin{array}{l}\text { generic five dimension Health Status EuroQol quality of life question- } \\
\text { naire }\end{array}$ \\
\hline $\mathrm{FI}$ & Fecal Incontinence \\
\hline GP & General Practitioner \\
\hline HRQOL & Health-Related Quality of Life \\
\hline HTA & Health Technology Assessment \\
\hline (i)CE & (incremental) Cost-Effectiveness \\
\hline ICERs & incremental Cost-Effectiveness Ratios \\
\hline ICIQ-UI SF & International Consultation on Incontinence Questionnaire Short Form \\
\hline ISLY & Incontinence Severity weighted Life Year \\
\hline MTA & Medical Technology Assessment \\
\hline MUI & Mixed Urinary Incontinence \\
\hline NICE & National Institute for Health and Clinical Excellence \\
\hline PRODISQ & modular PROductivity and DISease Questionnaire \\
\hline RCT & randomised controlled trial \\
\hline QALY & Quality Adjusted Life Year \\
\hline $\mathrm{QALY}_{\text {societal }}$ & $\begin{array}{l}\text { Quality Adjusted Life Year based on societal preferences for health } \\
\text { outcomes }\end{array}$ \\
\hline$Q A L Y_{\text {patient }}$ & $\begin{array}{l}\text { Quality Adjusted Life Year based on patient's preferences for health } \\
\text { outcomes }\end{array}$ \\
\hline SUI & Stress Urinary Incontinence \\
\hline UI & Urinary Incontinence \\
\hline UUI & Urgency Urinary Incontinence \\
\hline VAS & Visual Analogue Scale \\
\hline VOI analysis & Value Of Information analysis \\
\hline WTP & Willingness To Pay \\
\hline
\end{tabular}



Chapter 1

General Introduction 


\section{General Introduction}

In 1998, the WHO acknowledged the worldwide problem of urinary incontinence (UI). The prevalence of UI is considerable, and it seriously affects the health-related quality of life of adults, predominantly adult women. Incontinence becomes more prevalent with age, it is often complicated by co-morbidities and it is one of the most important reasons for the early admission of elderly people into hospitals or nursing homes. As the world's population ages, important public-health and clinical management implications have to be considered over the next decade to effectively prevent and manage this condition. ${ }^{1}$ Despite the high prevalence estimates and clear guidelines on the initial assessment and treatment of $\mathrm{UI}$ in primary care, it is an area of healthcare that remains unglamorous. ${ }^{1-3}$

Awareness and understanding of $\mathrm{UI}$ among the general public, health professionals and health service planners are poor compared to other non life-threatening conditions, as a result of which many people are currently receiving inadequate treatment. (Editorial comment on Buckley et al) ${ }^{4,5}$ Doubts about the quality, efficiency and organisation of $\mathrm{UI}$ care and the increasing socio-economic burden imposed by UI on the healthcare system, with costs estimated to be higher than for numerous other major medical conditions, mean that it is becoming ever more important to find solutions to improve all aspects of UI care. ${ }^{6-9}$

\section{Background}

$\mathrm{UI}$ is a symptom, not a disease, and is defined as 'the complaint of any involuntary leakage of urine'. ${ }^{10-13}$ The most common types of UI are stress UI (SUI, 'involuntary leakage on effort or exertion, or on sneezing or coughing'), urgency UI (UUI, 'involuntary leakage accompanied by or immediately preceded by urgency', 'a sudden compelling desire to pass urine which is difficult to defer') and mixed UI (MUI, 'involuntary leakage associated with urgency and also with exertion, effort, sneezing or coughing'). ${ }^{11}$

\section{Prevalence, incidence and natural history of urinary incontinence}

UI currently affects $8 \%$ of the world's population (346 million people). By 2018 , an estimated 423 million adults worldwide are predicted to be affected by UI. ${ }^{1} \mathrm{SUI}$ is predicted to be the most common UI type because of its high prevalence in women (167 million). UUI is expected to affect 60 million people (similar for men and women) and MUI 66 million (more women than men), while 131 million individuals will be affected by 'other' types of UI (i.e. UI without symptoms of UUI or SUI), with similar numbers expected for men and women. ${ }^{4,5}$ 
The current prevalence of $\mathrm{UI}$ and the estimated prevalence in 2018 are highest in Europe $\left(9.6 \%\right.$ and $10 \%$ respectively).$^{14}$ In the Netherlands, 1.6 million individuals may currently be affected by UI. Published prevalence figures for UI vary widely, which is explained by differences between studies in terms of the definition of UI, epidemiologic methodology, and demographic characteristics.

$\mathrm{UI}$ is a dynamic health problem. Recent prospective studies have provided data on the incidence of UI and its natural history (progression, regression and resolution). ${ }^{15-18}$ Literature on incidence and remission rates (natural history) of UI is still scarce, particularly among men. The annual incidence of any new UI in women ranges from $3 \%$ to $11 \%$, with the highest incidence occurring during pregnancy, and it increases with age. Rates of complete remission of UI range from $0 \%$ to $13 \%$, with the highest remission rates after pregnancy. ${ }^{18}$ Remission tends to increase with age, though not as consistently as incidence, ${ }^{5}$ but whether remission results from treatments is unclear. The estimated prevalence of $\mathrm{UI}$ in middle-aged and older women in the general population appears to be in the range of $30 \%$ to $60 \%$ (increasing with age). ${ }^{5,19}$ Younger and middle-aged women are more likely to report SUI, with a peak in the fifth decade, whereas older women report more UUI or MUI. ${ }^{19,20}$

The epidemiology of $\mathrm{UI}$ in men has not been investigated to the same extent as in women. It appears that the prevalence of $\mathrm{UI}$ in men is approximately half that in women, with a more steady increase in prevalence with age than in women. The distribution of UI subtypes differs between men and women, due to gender differrences of UI-related pathophysiology. Most studies have found a predominance of UUI in men $(40 \%-80 \%$ of cases), followed by MUI $(10 \%-30 \%)$ and a minority with SUI (<10\%). Most studies found a large proportion of other/unclassified types.

The wide range of published prevalences of $U I$ reflects differences in the scope of the definition of UI (ranging from UI occurring once during the last 12 months to Ul occurring several times per day or week), as well as in methodology (telephone interviews, mailed questionnaires, patient examinations) and in the demographics of the population studied. UI is associated with the Caucasian race, and limited data from twin studies suggest that there is a substantial genetic component to the condition. $^{21-23}$

\section{Burden of urinary incontinence}

\section{Burden on individuals with urinary incontinence}

UI has a profound negative impact on individual well-being and health-related quality of life, exceeding that of many co-morbid diseases (e.g. diabetes, stroke and arthritis in the hands and wrists). ${ }^{24,25}$ The impact of incontinence on individuals varies with age, gender, type of incontinence, individual differences in coping skills and the 
quality of social support. ${ }^{26}$ Although incontinence may affect the social, domestic, occupational and sexual lives and well-being of those affected, regardless of their age, the true emotional and social burdens are not easily measured. ${ }^{27-29}$ Not surprisingly, an increase in the severity of UI symptoms is associated with an increasing impact on health-related quality of life, with the discomfort of UI increasing to various degrees. ${ }^{29-31}$ The UI symptoms disrupt normal activity patterns and require coping strategies that further limit various aspects of normal life. ${ }^{27}$ Individuals may restrict travel and avoid exercise, while time that would otherwise be used for preferred activities is taken up by measures required to cope with UI, such as changing pads or clothing, and making frequent trips to the toilet. ${ }^{32}$ Shame and embarrassment associated with UI may lead to avoiding social contacts and result in isolation. ${ }^{33}$ Furthermore, individuals with UI often have concomitant and treatable symptoms that can be quite troublesome, including UI frequency, urgency, nocturia, pelvic organ prolapse and faecal incontinence. ${ }^{34-40}$ Finally, UI is associated with a $30 \%$ increase in functional decline, and a twofold increased risk of falls, depressive symptoms and nursing home placement. ${ }^{24,41-44} \mathrm{Few}$ objective data are available about the effect of incontinence on sexual, parent-child and sibling relationships. ${ }^{45}$, ${ }^{46}$ Stress may result when these relationships involve caregiving. ${ }^{26}$

\section{Caregiver burden}

Incontinence is associated with greater levels of unpaid informal caregiving by family or friends as well as paid formal care, for example to help with toileting. A major source of stress for caregivers is the physical and mental effort caused by the unpleasantness of dealing with incontinence. Despite the large numbers of informal caregivers, there has been little research examining the impact of incontinence on caregiver burden. The dramatic increase in the numbers of baby boomers who are now faced with caring for their elderly parents will affect the kind of care arrangements they will choose for themselves when they become the elderly. ${ }^{26}$ Efforts to avoid nursing home placement are generating many creative ways to 'age in place'. The need for care, informal or formal, will have to be factored into these efforts. After adjustment for co-morbid illness, socio-economic status and living situation, older individuals who have UI require more informal care than those who are continent. Although women have a higher prevalence of incontinence, men receive more hours of informal caregiving than women, in each incontinence category. ${ }^{26}$ When informal caregivers can no longer cope with the additional burden imposed by incontinence, the likelihood of transfer to a nursing home increases. This is especially true when $\mathrm{UI}$ is accompanied by faecal incontinence. ${ }^{35,36}$ It is difficult to separate the burden of incontinence from the burden of other, co-existing conditions, especially dementia. ${ }^{26}$ 
Costs

The high prevalence of $\mathrm{UI}$ also results in substantial and well-documented economic consequences. In the Netherlands, absorbent pads (reimbursed by insurance companies) have for many years now been the most costly of all healthcare aids ( $€ 157$ million in 2009). ${ }^{47-49}$ Over the years, the numbers of users of absorbent pads increased (with a $14 \%$ increase in 2009 compared to 2008 , to a total of 654,000 users). Dutch UI costs are in line with those elsewhere in the world: annual direct costs of UI in the United States (primarily costs of routine care, including out-ofpocket costs) are estimated to be over $\$ 20$ billion ( $€ 14$ billion), ${ }^{50}$ which is more than the annual costs for breast, cervical, uterine and ovarian cancer combined. ${ }^{9}$ Substantial indirect costs from work loss and inability to do volunteer work have also been reported. ${ }^{51}$ It is often assumed that incontinence pads are necessary and are prescribed in addition to the recommended/necessary treatment. However, given the magnitude of this health problem and the high costs of pads, many $\mathrm{UI}$ patients may get only prescriptions for pads from their general practitioner (GP) or even directly from the GP's assistant. UI patients may not receive proper consultation or diagnostics and treatment, while their need for pad use and their adherence to advice on bladder and/or pelvic floor muscle training may not be sufficiently monitored. ${ }^{52,53}$ This would imply a large number of patients with suboptimal care and unnecessarily high expenditure for incontinence pads. With the increased ageing of the population, the annual socio-economic burden is likely to increase further. $^{18,36,54-59}$

\section{Possible causes of the burden of urinary incontinence}

\section{Risk and prognostic factors}

$\mathrm{UI}$ is associated with a range of risk factors. In some instances, there is a single clear cause, but in most cases, the pathways are much more complex, they involve multiple factors and are still not well understood. There is evidence that some risk factors for UI are modifiable, and that the likelihood of UI developing or worsening can be reduced through preventive measures or treatment. However, the evidence is not strong, is inconsistent, and is insufficiently focused on specific types of incontinence. Below I summarise the most credible general results. Age is strongly associated with an increased risk of UI, both in women and in men. ${ }^{19,30,60}$ Pregnancy and vaginal delivery are relevant risk factors, though they become less important with increasing age. Contrary to what used to be popularly believed, menopause per se does not appear to be a risk factor for UI, and there is conflicting evidence regarding hysterectomy. Statistically significant relationships have been found for race and 
ethnicity (white women have higher rates of incontinence) and for family history in women. ${ }^{21-23,26}$ Diabetes mellitus has been found by most studies to be a risk factor, while oral oestrogen substitution and body mass index are important modifiable risk factors for UI. ${ }^{19,61-64}$ Various studies have reported UI to be associated with depression, cognitive impairment, impaired physical function, restricted mobility and neurological disorders. ${ }^{19,65}$ However, these associations are sometimes weak and the causal relationships are unclear. ${ }^{19}$ Other potential risk factors, including smoking, diet, urinary tract infections, mild UI, constipation, faecal incontinence, genital prolapse, congestive heart failure, use of diuretics and childhood enuresis have been reported to be associated with $\mathrm{UI}$, but have not been established as etiologic risk factors. ${ }^{19,37,38}$ Although $\mathrm{UI}$ is a chronic dynamic condition with a strong tendency to worsen over time, few risk factors for change in incontinence status have been identified. $^{66}$

Risk factors in men have not yet been fully described, but might include the presence of lower urinary tract symptoms (LUTS), urinary tract infections, functional and cognitive impairment, neurological disorders and prostatectomy. ${ }^{19}$

\section{Help-seeking behaviour}

Despite the impact that UI can have on the health-related quality of life, many people never seek help for their incontinence, and fewer than half of incontinent individuals spontaneously report their symptoms during consultations. ${ }^{26,60,67-69} \mathrm{UI}$ thus remains a largely hidden and often unreported problem. Clinical recognition of $U I$ is hampered by multiple factors, including social stigma, perception of the condition and discomfort about disclosing the symptoms, limited knowledge about potential benefits of intervention, the use of non-specific language by patients to des-cribe their concerns and care requirements to care providers, competing demands and time limitations during consultations, poor continuity of care and lack of consistency and clarity when care providers seek to determine continence status. ${ }^{5,53,69-75}$ Factors associated with help-seeking are age, duration and severity of UI symptoms, urgency/mixed type of UI and having spoken to others. ${ }^{19,53,71,73,76-78}$ Men tend to be less proactive in their health seeking behaviour, so gender-specific strategies to address this should be considered. ${ }^{79}$ Findings on gender differences in the frequency of help-seeking behaviour for lower urinary tract symptoms have been conflicting. ${ }^{73,80,81}$

Only a small proportion of UI community-residing people have been treated with surgery, exercise regimens or medication; the major method of actively managing the $\mathrm{Ul}$ population is currently the use of absorbent products. ${ }^{19}$ In addition to seeking help from the formal healthcare system, common responses to symptoms of illness are self-management and self-treatment behaviour and receiving informal care by family and friends, for example in the form of toileting assistance. ${ }^{26}$ 


\section{How to reduce the burden of illness?}

\section{Interventions and existing guidelines}

National and international UI guidelines indicate that conservative management is the main approach to initial care for $\mathrm{UI}$ in both men and women. Conservative management consists of appropriate advice on potentially modifiable lifestyle factors, together with bladder and pelvic floor muscle training, all of which require a change of behaviour. ${ }^{6,56,59,82-84}$ Although some of these recommendations are based on a good and consistent level of evidence, many others are based on insufficient evidence and are essentially hypotheses requiring further testing in high quality studies. Weight loss, ${ }^{64}$ smoking cessation, control of chronic pulmonary disease, timed voiding, bladder training and oral fluid management may reduce the risk of leakage but require continued adherence to the necessary adjustments to maintain longterm response. Pelvic floor muscle training also requires long-term adherence to the home training programme. These interventions have few or no adverse effects and entail low costs, so most patients with mild to moderate complaints prefer to try such less invasive treatments first. Lifestyle advice includes a suitable choice and efficient use of absorption pads, and is part of an integral behavioural strategy to improve quality of life. Just as for most health problems, GPs tend to be the gatekeepers for this (at least in the Netherlands). ${ }^{85-87}$ Despite GP guidelines, effective treatment is currently not being consistently offered. ${ }^{4,60,88,89}$ Patients tend to interpret their GP's lack of response as an admission that no treatment is available. ${ }^{88}$ It has been suggested that many primary healthcare providers lack confidence in managing $\mathrm{UI}$ and that this contributes to insufficient treatment of those seeking help. ${ }^{90}$ Also, there are serious doubts about sufficient dissemination and implementation of UI clinical practice guidelines. ${ }^{6,56,59,89,91,92}$ A committee of the Dutch Health Council concluded that there is insufficient knowledge about typical primary UI care, and that no information is available about adherence to existing standards and guidelines in routine practice. ${ }^{6}$

\section{Role revision: involving nurse specialists}

Since 2002, there has been a growing emphasis on involving nurse specialists in general practice care, with positive findings for chronic disorders. Revision of professional roles is seen as a viable strategy to consider when addressing challenges facing healthcare over the coming decades. ${ }^{93-97}$ Quality of healthcare can be threatened by shortages of medical staff, variations in service access and quality, pressure to contain costs, an ageing population, new technologies and higher demands. The available evidence suggests that non-physician staff working either as substitutes for physicians in specific areas of care or as a supplement to physician care can 
maintain and, for some aspects, even improve the quality of care and the outcomes for patients. Revision of roles appears to be acceptable to patients as well as physicians. Nevertheless, Laurant concluded in her review of role revision ${ }^{98}$ that evidence on role revision in general is as yet too limited to draw firm conclusions, particularly regarding the economic impacts and cost-effectiveness. When we started our research in this area, only one systematic literature review had analysed the effect of UI treatment by nurses on clinical and economic outcomes. ${ }^{99}$ The review included a total of 12 RCTs, varying in terms of population, setting, outcome measurement and control/intervention groups. The authors concluded that there was limited evidence that treatment by nurses resulted in a decrease in UI. Most of the evidence was derived from research with follow-up periods of less than one year. No evidence was found for cost reduction, and no economic evaluations from the societal perspective had been performed. The review suggested that nurses might improve health outcomes and satisfaction among UI patients. Although the evidence was limited and weak, we concluded from the literature data that nurse specialists might be well accepted by patients and GPs, and could represent a feasible and useful option to change the organisation of UI care for effective and efficient UI management and treatment.

Nurse specialists have specific expertise, are used to following protocols, spend more time motivating patients, are familiar with emotional support for patients and their informal caregivers regarding behavioural change, and offer a low threshold service. ${ }^{26,98}$ Another argument for the use of nurse specialists is that the complexity of UI should not be underestimated. UI is not always a straightforward condition, for two reasons. First, as outlined above, there are large variations with regard to how, when and why men and women with UI seek help, and the kind of help they expect, prefer or prioritise. Second, UI is frequently complicated by pelvic floor related dysfunctions (e.g. pelvic organ prolapse, faecal incontinence) and comorbidities, particularly among the elderly. Incorporating sufficiently trained nurse specialists, modelled on nurse specialists for diabetes and chronic pulmonary diseases, may also offer a solution to the current inadequate UI care, including the inappropriate prescribing of incontinence pads. Therefore, nurse specialists may be well equipped, after additional training, and using their specific expertise on UI, to support GPs after the latter have defined the initial problem.

\section{Aims and approach of the present project}

The main objective of the research reported on in this thesis was to study whether the involvement of nurse specialists in primary $\mathrm{UI}$ care is feasible and leads to favourable effects on the outcomes and cost-effectiveness of care for adult UI patients, compared to care-as-usual. We assessed this construct of UI care by follo- 
wing the steps in the model developed by Tugwell, which is frequently used in health technology assessment (HTA). ${ }^{100}$ The most important characteristic of HTA is that it focuses on effectiveness and at least one other aspect of healthcare (for example costs, organisational aspects or quality of care), with the aim of providing input for decision making in policy and practice. ${ }^{101}$ This suggests that several different types of indicators have to be measured to provide meaningful, precise and timely information for various stakeholders about whether to implement innovations in healthcare. These aspects are especially important given the challenges facing healthcare. ${ }^{93-97}$ Furthermore, HTA is an iterative process, and the assessment needs to continue throughout the lifespan of a technology. This iterative process is illustrated by the 'technology assessment iterative loop' (TAIL), which is represented in Figure $1 .{ }^{100}$ We used this seven-step loop approach to guide our choice of quantitative and qualitative research methods in this project to evaluate the various aspects of primary care for UI patients. ${ }^{102,103}$ This general introductory chapter, based on the literature, has described the burden of illness presented by UI (step 1 of TAIL), partially introduced and identified potential causes of the burden of UI (step 2) and discussed potentially feasible and effective interventions to reduce the burden of UI (step 3). The other chapters of this thesis reflect subsequent steps in TAIL: causes of the burden of illness (step 2), effectiveness (step 3), efficiency (step 4) and, partly, the fifth step of synthesis and implementation. TAIL emphasises that the assessment of an intervention is not finished when the intervention is implemented, but that the intervention should be continuously monitored and reassessed. By assessing whether the intervention has reduced the burden of illness, one will eventually arrive back at the first step in the cycle, whereupon the cycle is repeated. 


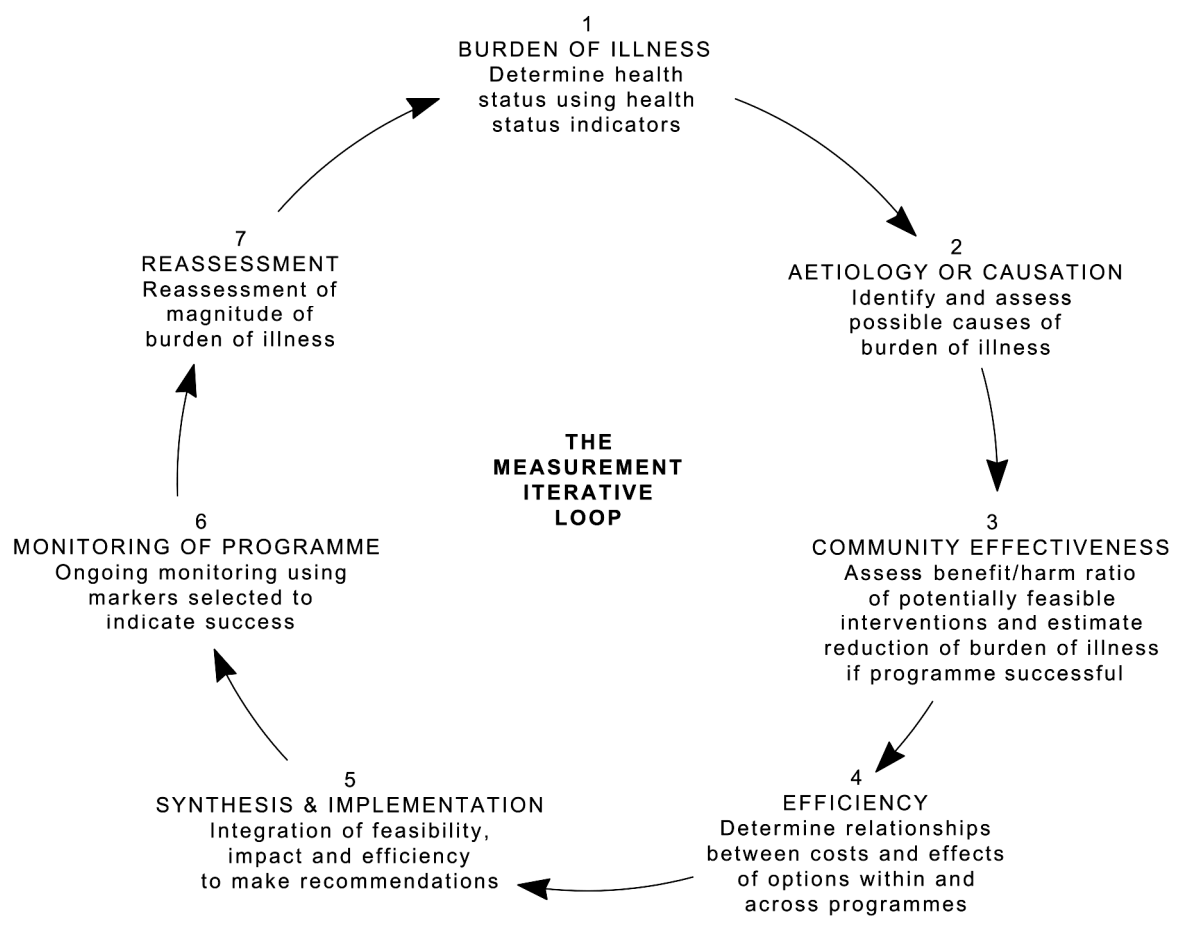

Figure 1 Technology assessment iterative loop (TAIL).

\section{Objectives, methods and research questions of the studies}

Firstly, the paucity of available data on typical UI care for adults ${ }^{6}$ induced us to study current care-as-usual for UI patients in primary care. This was done by means of two cross-sectional postal surveys in two Dutch regions. One survey was held among GPs, to assess the level of adherence or non-adherence of GPs to the recommendations on diagnosis, evaluation and treatment presented in the Guideline on Urinary Incontinence of the Dutch College of General Practitioners (GLUI). ${ }^{2,3}$ The other survey was performed in the same areas and the same period, among adult UI patients who had received a first-time prescription for incontinence pads in the preceding year. Care-as-usual was studied in this specific patient group because it is often assumed that pads are necessary and are prescribed in addition to the recommended/necessary treatment. We aimed to identify important issues for GPs and patients in UI care, to distinguish barriers and facilitators for the improvement of UI care.

The studies thus addressed the following two research questions concerning current UI care-as-usual: 
- To what extent do general practitioners adhere to their Guideline on Urinary Incontinence?

- What are the reasons for general practitioners to adhere or not adhere to components of the Guideline?

- Is adherence related to specific characteristics of the general practitioners or the patients, or to logistical problems?

- What kind of initial management do patients with urinary incontinence receive from their general practitioner and is this management related to patient characteristics?

- Are pad users satisfied with the care offered by general practitioners and is their satisfaction related to patient characteristics and the type of care received?

In line with recommendations by the Dutch Health Council, ${ }^{6}$ we also wanted to study the effectiveness, efficiency and feasibility of involving specially trained home care and hospital-linked nurse specialists to work in general practice, on the basis of clear and accepted work agreements/protocols, to assist in the treatment of adult patients with $\mathrm{UI}$, and to compare this with care-as-usual. ${ }^{2,3}$ To approximate the normal caseload and care-as-usual in general practice as much as possible, ${ }^{104}$ we performed a multicentre pragmatic randomised controlled trial (RCT), called Promoting Continence Study (PromoCon Study). The study addressed the following research question about the effectiveness of the intervention:

- Does the involvement of a nurse specialist in the management of adult patients with urinary incontinence reduce the overall severity of urinary incontinence, including symptoms of frequency and volume and the impact on the healthrelated quality of life, compared to care-as-usual?

Alongside this RCT, we carried out an economic evaluation from a societal perspective, addressing the following research question:

- What is the cost-effectiveness of involving a nurse specialist in the management of adults with urinary incontinence in primary care, compared to care-as-usual provided by general practitioners?

In addition to investigating effectiveness and cost-effectiveness, we tried to understand what causes the effect, especially because we foresaw possible difficulties with the implementation of our intervention, given the 2006 reforms of the Dutch healthcare system. ${ }^{87,97}$ Although role revision from GPs to nurses is increasing, we did not know how the nurses experienced their task and how consumers (i.e. patients and GPs) would perceive and experience the care provided by this new type of healthcare professional. This relates to our third aim, namely examining the feasibility of the intervention. To this end, we performed a qualitative focus group study with the nurse specialists who participated in the trial, to explore how the 
nurses perceived their role in the care for UI patients and how they had performed the intervention. In addition, a postal questionnaire was sent out to the UI patients to assess patient satisfaction in relation to relevant themes in the care for UI patients according to UI guidelines. Finally, a convenience sample of the participating GPs was interviewed in a 10-minute structured interview by telephone, after the study to also explore GPs' experiences with and attitudes towards the intervention by nurse specialists, and their views on the value of this new form of care. This was intended to explain potential effects and to identify facilitators and barriers for wider implementation. The following research question was addressed for the nurse specialists:

- What are the experiences and attitudes of the nurse specialists regarding their role in the care for patients with urinary incontinence?

For the UI patients and the GPs, we addressed the following research question:

- What are the perceptions and experiences of consumers, that is, patients with urinary incontinence and GPs, regarding the care provided by the new type of healthcare professional, the nurse specialist?

\section{Outline of this thesis}

The answers to the first two research questions, about care-as-usual, are presented in Part I, comprising the next two chapters of this thesis.

Chapter 2 presents the findings of the survey among GPs on the level of adherence or non-adherence to the Guideline on Urinary Incontinence of the Dutch College of General Practitioners (GLUI)., ${ }^{2,3}$

Chapter 3 reports the results of the survey among UI patients on the kind of initial management that $U$ I patients had received from their GP during the past 12 months and their satisfaction with the GP care they had received.

Part II of this thesis relates to the PromoCon study, a multicentre pragmatic randomised controlled trial comparing the intervention of the trained nurse specialists with care-as-usual.

Chapter 4 describes the PromoCon study protocol, presenting information on the training and assessment of the nurse specialists prior to the trial, and the nurse intervention protocol.

Chapter 5 reports on the effectiveness of the intervention by nurse specialists on the patient-reported outcome of UI severity, including symptoms of frequency and volume and the impact of UI on health-related quality of life (ICIQ-UI SF), after three months and after one year.

The results of the economic evaluation from the societal perspective alongside the RCT are presented in Chapter 6. This study measured the costs of healthcare (use of incontinence pads, GP consultations), patient and family costs and produc- 
tivity costs, using general (Quality Adjusted Life Year [QALY]) and disease specific (Incontinence Severity weighted Life Year [ISLY]) outcomes, with a 12-month time horizon. Results are presented as Cost-Effectiveness Planes and Cost-Acceptability Curves, supplemented by a Value Of Information (VOI) analysis determining an Expected Value of Perfect Information (EVPI).

Chapter 7 reports on the experiences and attitudes of the nurse specialists from the focus group study.

Chapter $\mathbf{8}$ describes the perceptions and experiences of consumers (i.e. patients and GPs) with the care provided by this new type of healthcare professional.

Finally, our main findings and their implications for practice, policy and further research on the management of UI patients in general practice are discussed in the General Discussion in Chapter 9. 


\section{References}

1. Abrams P. Assessment and treatment of urinary incontinence. Lancet. 2000;355:2153-8.

2. Lagro-Janssen A, Breedveldt-Boer H, Dongen Jv, Lemain T, Steeneken F, Dijkstra R, et al. NHGStandaard Incontinentie voor Urine [Dutch College Guideline on Urinary Incontinence]. Huisarts Wet. 1995;38(2):71-80.

3. Lagro-Janssen ALM, Teunissen D, Breedveldt-Boer HP, Dongen van J, Lemain TJJ, Steeneken F, et al. NHG-Standaard Incontinentie voor Urine M46 Eerste herziening [Dutch College Guideline on Urinary Incontinence. First revision]. Huisarts Wet. 2006;49(2):501-10.

4. Gasquet I, Tcherny-Lessenot S, Gaudebout P, Bosio Le Goux B, Klein P, Haab F. Influence of the Severity of Stress Urinary Incontinence on Quality of Life, Health Care Seeking, and Treatment: A National Cross-Sectional Survey. Eur Urol. 2006;50(4):818-25.

5. Buckley BS, Lapitan MCM. Prevalence of Urinary Incontinence in Men, Women, and Children-Current Evidence: Findings of the Fourth International Consultation on Incontinence. Urology. 2010;76(2):265-70.

6. Health Council of the Netherlands (Gezondheidsraad). Urine Incontinentie [Urinary Incontinence]. Publication no. 2001/12. The Hague 2001. Report No.: ISBN: 90-5549-375-9.

7. Health Insurance Board (CVZ). Urine Incontinentie bij volwassenen [Urinary Incontinence in adults]. Diemen 2003 Contract No.: 2003/147.

8. Health Insurance Board (CVZ). Attentie voor incontinentie [Attention for incontinence]. Amstelveen 2006.

9. Subak LL, Brown JS, Kraus SR, Brubaker L, Lin FM, Richter HE, et al. The "Costs" of Urinary Incontinence for Women. Obstet Gynecol. 2006;107(4):908-16.

10. Abrams P, Blaivas J, Stanton S, Andersen J. Standardization of terminology of lower urinary tract function. The International Continence Society Committee on Standardization of Terminology. Neurourol Urodynam. 1988;7:403-27.

11. Abrams P, Cardozo L, Fall M, Griffits D, Rosier P, Ulmsten U, et al. The standardisation of Terminology of Lower Urinary Tract function: Report from the Standardisation Sub-committee of the International Continence Society. Neurourol Urodynam. 2002;21:167-78.

12. Abrams P, Artibani W, Cardozo L, Dmochowski R, Kerrebroeck PV, Sand P. Reviewing the ICS 2002 Terminology Report: The Ongoing Debate. Neurourol Urodynam. 2006;25:293.

13. Haylen BT, De Ridder D, Freeman RM, Swift SE, Berghmans B, Lee J, et al. An international urogynecological association (IUGA)/international continence society (ICS) joint report on the terminology for female pelvic floor dysfunction. Neurourol Urodynam. 2010;29(1):4-20.

14. Irwin DE, Kopp ZS, Agatep B, Milsom I, Abrams P. Worldwide prevalence estimates of lower urinary tract symptoms, overactive bladder, urinary incontinence and bladder outlet obstruction. BJU Int. 2011;10.1111/j.1464-410X.2010.09993.x.

15. Long RM, Giri SK, Flood HD. Current concepts in female stress urinary incontinence. The Surgeon. 2008;6(6):366-72.

16. Offermans MPW, Du Moulin MFMT, Hamers JPH, Dassen T, Halfens RJG. Prevalence of urinary incontinence and associated risk factors in nursing home residents: A systematic review. Neurourol Urodynam. 2009;28(4):288-94.

17. Botlero R, Davis SR, Urquhart DM, Shortreed S, Bell RJ. Age-specific prevalence of, and factors associated with, different types of urinary incontinence in community-dwelling Australian women assessed with a validated questionnaire. Maturitas. 2009;62(2):134-9.

18. Wennberg A-L, Molander U, Fall M, Edlund C, Peeker R, Milsom I. A Longitudinal Population-based Survey of Urinary Incontinence, Overactive Bladder, and Other Lower Urinary Tract Symptoms in Women. Eur Urol. 2009;55(4):783-91. 
19. Milsom I, Altman D, Lapitan M, Nelson R, Sillén N, Thom D. Committee 1. Epidemiology of Urinary (UI) and Faecal (FI) Incontinence and Pelvic Organ Prolapse (POP). In: Abrams P, Cardozo L, Khoury S, Wein A, editors. Incontinence 4th International Consultation on Incontinence Paris July 5-8, 2008. Plymouth: Health Publication Ltd; 2009. p. 63, 72, 89-92.

20. Minassian V, Stewart W, Wood G. Urinary incontinence in women: variation in prevalence estimates and risk factors. Obstet Gynecol. 2008;111(2, Part 1):324-31.

21. Rohr G, Kragstrup J, Gaist D, Christensen K. Genetic and environmental influences on urinary incontinence: a Danish population-based twin study of middle-aged and elderly women. Acta Obstet Gynecol Scand. 2004;83:978-82.

22. Dietz HP, Hansell NK, Grace ME, Eldridge AM, Clarke B, Martin NG. Bladder neck mobility is a heritable trait. BJOG. 2005;112(3):334-9.

23. Altman D, Forsman $M$, Falconer $C$, Lichtenstein $P$. Genetic influence on stress urinary incontinence and pelvic organ prolapse. Eur Urol. 2008;54:918-23.

24. Holroyd-Leduc JM, Mehta KM, Covinsky KE. Urinary Incontinence and Its Association with Death, Nursing Home Admission, and Functional Decline. J Am Geriatr Soc. 2004;52(5):712-8.

25. Ko Y, Lin S, Salmon J, Bron M. The Impact of Urinary Incontinence on Quality of Life of the Elderly. Am J Manag Care. 2005;11:S103-S11.

26. Landefeld CS, Bowers BJ, Feld AD, Hartmann KE, Hoffman E, Ingber MJ, et al. National Institutes of Health State-of-the-Science Conference Statement: Prevention of Fecal and Urinary Incontinence in Adults. Ann Intern Med. 2008;148(6):449-58.

27. Fultz N, Fisher G, Jenkins K. Does urinary Incontinence Affect Middle-Aged and Older Women's Time Use and Activity Patterns? Obstet Gynecol. 2004;104(6):1327-34.

28. Hampel C, Artibani W, Espuña Pons M, Haab F, Jackson S, Romero J, et al. Understanding the Burden of Stress Urinary Incontinence in Europe: A Qualitative Review of the Literature. Eur Urol. 2004;46(1):15-27.

29. Papanicolaou S, Hunskaar S, Lose G, Sykes D. Assessment of bothersomeness and impact on quality of life of urinary incontinence in women in France, Germany, Spain and the UK. BJU Int. 2005;96:831-8.

30. Hannestad YS, Rortveit G, Sandvik H, Hunskaar S. A community-based epidemiological survey of female urinary incontinence: The Norwegian EPINCONT Study. J Clin Epidemiol. 2000;53(11):1150-7.

31. Melville JL, Miller EA, Fialkow MF, Lentz GM, Miller JL, Fenner DE. Relationship between patient report and physician assessment of urinary incontinence severity. Am J Obstet Gynecol. 2003;189(1):76-80.

32. Diokno A, Burgio K, Fultz N, Kinchen K, Obenchain R, Bump R. Medical and self-care practices reported by women experiencing urinary incontinence. Am J Manag Care. 2004;10(2):69-78.

33. Monz B, Pons M, Hampel C, Hunskaar S, Quail D, Samsioe G, et al. Patient-reported impact of urinary incontinence. Results from treatment seeking women in 14 European countries. Maturitas. 2005;30(52):suppl 2; 24-34.

34. MacLennan AH, Taylor AW, Wilson DH, Wilson D. The prevalence of pelvic floor disorders and their relationship to gender, age, parity and mode of delivery. BJOG-Int J Obstet Gy. 2000;107(12):1460-70.

35. Barry MJ, Link CL, McNaughton-Collins MF, McKinlay JB, for the Boston Area Community Health Investigators. Overlap of different urological symptom complexes in a racially and ethnically diverse, community-based population of men and women. BJU Int. 2008;101(1):45-51.

36. Nygaard I, Barber MD, Burgio KL, Kenton K, Meikle S, Schaffer J, et al. Prevalence of Symptomatic Pelvic Floor Disorders in US Women. JAMA. 2008;300(11):1311-6.

37. Slieker-ten Hove M, Pool-Goudzwaard A, Eijkemans M, Steegers-Theunissen R, Burger C, Vierhout M. The prevalence of pelvic organ prolapse symptoms and signs and their relation with bladder and bowel disorders in a general female population. Int Urogynecol J. 2009;20(9):1037-45.

38. Slieker-ten Hove MCP, Pool-Goudzwaard AL, Eijkemans MJC, Steegers-Theunissen RPM, Burger CW, Vierhout ME. Prevalence of double incontinence, risks and influence on quality of life in a general female population. Neurourol Urodynam. 2010;29(4):545-50. 
39. de Boer T, Slieker-ten Hove M, Burger C, Vierhout M. The prevalence and risk factors of overactive bladder symptoms and its relation to pelvic organ prolapse symptoms in a general female population. Int Urogynecol J. 2011;22(5):569-75.

40. de Boer TA, Slieker-ten Hove MCP, Burger CW, Kluivers KB, Vierhout ME. The prevalence and factors associated with previous surgery for pelvic organ prolapse and/or urinary incontinence in a crosssectional study in The Netherlands. Eur J Obstet Gyn R B. 2011;In Press, Corrected Proof.

41. Thom DH, Haan MN, Van Den Eeden SK. Medically recognized urinary incontinence and risks of hospitalization, nursing home admission and mortality. Age Ageing. 1997;26(5):367-74.

42. Brown J, McGhan W, Chokroverty S. Comorbidities associated with overactive bladder. Am J Manag Care. 2000;6:S574-9.

43. Kron M, Loy S, Sturm E, Nikolaus T, Becker C. Risk Indicators for Falls in Institutionalized Frail Elderly. Am J Epidemiol. 2003;158(7):645-53.

44. Hägglund D, Walker-Engström M-L, Larsson G, Leppert J. Reasons why women with long-term urinary incontinence do not seek professional help: a cross-sectional population-based cohort study. Int Urogynecol J. 2003;14(5):296-304.

45. Coyne K, Sexton C, Irwin D, Kopp Z, Kelleher C, Milsom I. The impact of overactive bladder, incontinence and other lower urinary tract symptoms on quality of life, work productivity, sexuality and emotional well-being in men and women: results from the EPIC study. BJU Int. 2008;101(11):1388-95.

46. Bekker MD, Beck JJH, Putter H, Van Driel MF, Pelger RCM, Weijmar Schultz WC, et al. Sexual Experiences of Men with Incontinent Partners. J Sex Med. 2010;7(5):1877-82.

47. Health Insurance Board (CVZ). GIP-peilingen. Totale kosten 2001-2005, hulpmiddelencategorie A: Verzorgingsmiddelen. 2006.

48. Health Insurance Board (CVZ). GIP-peilingen. Total cost 2002-2006. 2007.

49. Health Insurance Board (CVZ). GIP-peilingen 2009. Ontwikkelingen genees- en hulpmiddelengebruik. 2010 September; $\mathrm{nr} 31$.

50. Wilson L, Brown J, Shin G, Luc K, Subak L. Annual direct cost of urinary incontinence. Obstet Gynecol. 2001;98(398-406).

51. Thom DH, Nygaard IE, Calhoun EA. Urologic diseases in America project: urinary incontinence in women; National trends in hospitalizations, office visits, treatment and economic impact. J Urol. 2005;173(4):1295-301.

52. Teunissen TAM, Bosch van den W, Weel van C, Lagro-Janssen ALM. Urinary incontinence in the elderly: attitudes and experiences of general practitioners. A focus group study. Scand J Prim Health Care. 2006;24(1):56-61.

53. Shaw C, Gupta RD, Wlilliams KS, Assassa RP, McGrother C. A survey of help-seeking and treatment provision in women with stress urinary incontinence. BJU Int. 2006;97(4):752-7.

54. Teunissen TAM, Bosch van den WJHM, Hoogen van den HJM, Lagro-Janssen ALM. Prevalence of urinary, fecal and double incontinence in the elderly living at home. Int Urogynecol J 2004;15(1):10-3.

55. DeLancey J. The hidden epidemic of pelvic floor dysfunction: Achievable goals for improved prevention and treatment. Am J Obstet Gynecol. 2005;192(5):1488-95.

56. Norton P, Brubaker L. Urinary incontinence in women. Lancet. 2006;367(9504):57-67.

57. Viktrup L, Lose G. Incidence and Remission of Lower Urinary Tract Symptoms During 12 Years After the First Delivery: A Cohort Study. J Urol. 2008;180(3):992-7.

58. Bodenheimer T, Chen E, Bennett D. Confronting The Growing Burden Of Chronic Disease: Can The U.S. Health Care Workforce Do The Job? Health Aff. 2009;28(1):64-74.

59. Abrams P, Andersson KE, Birder L, Brubaker L, Cardozo L, Chapple C, et al. Fourth international consultation on incontinence recommendations of the international scientific committee: Evaluation and treatment of urinary incontinence, pelvic organ prolapse, and fecal incontinence. Neurourol Urodynam. 2010;29(1):213-40.

60. Hunskaar S, Burgio K, Diokno A, Herzog A, Hjalmas K, Lapitan M. The prevalence of urinary incontinence in women in four European countries. BJU Int. 2004;93(3):324-30. 
61. Rortveit G, Hannestad YS, Daltveit AK, Hunskaar S. Age- and type-dependent effects of parity on urinary incontinence: the Norwegian EPINCONT study. Obstet Gynecol. 2001;98(6):1004-10.

62. Lifford KL, Curhan GC, Hu FB, Barbieri RL, Grodstein F. Type 2 Diabetes Mellitus and Risk of Developing Urinary Incontinence. J Am Geriatr Soc. 2005;53(11):1851-7.

63. Press JZ, Klein MC, Kaczorowski J, Liston RM, Von Dadelszen P. Does Cesarean Section Reduce Postpartum Urinary Incontinence? A Systematic Review. Birth. 2007;34(3):228-37.

64. Subak LL, Wing R, West DS, Franklin F, Vittinghoff E, Creasman JM, et al. Weight loss to treat urinary incontinence in overweight and obese women. N Engl J Med. 2009;360(5):481-90.

65. Melville JL, Fan M-Y, Rau H, Nygaard IE, Katon WJ. Major depression and urinary incontinence in women: temporal associations in an epidemiologic sample. Am J Obstet \& Gynecol. 2009;201: 490.e1-7.

66. Thom DH, Brown JS, Schembri M, Ragins Al, Subak LL, Eeden SKvd. Incidence of and Risk Factors for Change in Urinary Incontinence Status in a Prospective Cohort of Middle-Aged and Older Women: The Reproductive Risk of Incontinence Study in Kaiser. J Urol. 2010;184(41):394-401.

67. Hannestad YS, Rortveit G, Hunskaar S. Help-seeking and associated factors in female urinary incontinence. Scan J Prim Health Care. 2002;20(20):102-7.

68. Hunskaar S, Burgio K, Diokno A, Herzog A, Hjalmas K, Lapitan M. Epidemiology and natural history of urinary incontinence in women. Urology. 2003;62(4 Suppl 1):16-23.

69. Koch L. Help-Seeking Behaviors of Women with Urinary Incontinence: An Integrative Literature Review. J Midwifery Women Health. 2006;51(6):e39-e44.

70. Peake S, Manderson L, Potts H. 'Part and parcel of being a woman': female urinary incontinence and constructions of control. Med Anthropol Q. 1999;13:267-85.

71. Shaw C, Tansey R, Jackson C, Hyde C, Allan R. Barriers to help seeking in people with urinary symptoms. Fam Pract. 2001;18(1):48-52.

72. Wolters R, Wensing M, Van Weel C, Van Der Wilt GJ, Grol RPTM. Lower urinary tract symptoms: social influence is more important than symptoms in seeking medical care. BJU Int. 2002;90(7):655-61.

73. Teunissen D, Lagro-Janssen T. Urinary incontinence in community dwelling elderly: are there sex differences in help-seeking behaviour? Scand J Prim Health Care. 2004;22(4):209 - 16.

74. Hägglund D, Wadensten B. Fear of humiliation inhibits women's care-seeking behaviour for longterm urinary incontinence. Scand J Caring Sci. 2007;21(3):305-12.

75. Melville JL, Wagner LE, Fan M-Y, Katon WJ, Newton KM. Women's Perceptions about the Etiology of Urinary Incontinence. J Womens Health. 2008;17(7):1093-8.

76. Teunissen TAM, Weel van C, Lagro-Janssen ALM. Urinary incontinence in older people living in the community examining helpseeking behaviour. Br J Gen Pract. 2005;55:776-82.

77. O'Donnell M, Lose G, Sykes D, Voss S, Hunskaar S. Help-Seeking Behaviour and Associated Factors among Women with Urinary Incontinence in France, Germany, Spain and the United Kingdom. Eur Urol. 2005;47(3):385-92.

78. Irwin D, Milsom I, Kopp Z, Abrams P. Symptom bother and health care-seeking behavior among individuals with overactive bladder. Eur Urol. 2008;53:1029-39.

79. Harris M, McKenzie S. Mens's health: what's a GP to do? Med J Aust. 2006;185(8):440-4.

80. Diokno A, Estanol M, Ibrahim I, Balasubramaniam M. Prevalence of urinary incontinence in community dwelling men: a cross sectional nationwide epidemiological survey. Int Urol Nephrol. 2007;39(1):129-36.

81. Teunissen TAM, Lagro-Janssen ALM. Sex differences in the use of absorbent (incontinence) pads in independently living elderly people: do men receive less care? Int J Clin Pract. 2009;63(6):869-73.

82. Teunissen $T$, Jonge de A, Weel van C, Lagro-Janssen ALM. Treating urinary incontinence in the elderly-conservative measures that work: A systematic review. Fam Pract. 2004;53(1):25-30, 2.

83. Statline database.2010. Last update: 10-12-2009; numbers of 1-1-2009; [database on the Internet] [cited 02-02-2010]. Available from: http://statline.cbs.nl/StatWeb/publication/. 
84. Sherburn M, Bird M, Carey M, Bø K, Galea MP. Incontinence improves in older women after intensive pelvic floor muscle training: An assessor-blinded randomized controlled trial. Neurourol Urodynam. 2011;30(3):317-24.

85. Gerwen van M, Schellevis F, Lagro-Janssen T. Management of urinary incontinence in general practice: data from the Second Dutch National Survey. J Eval Clin Pract. 2009;15: 341-5.

86. Buckley B, Lapitan M. Prevalence of urinary and faecal incontinence and nocturnal enuresis and attitudes to treatment and help-seeking amongst a community-based representative sample of adults in the United Kingdom. Int J Clin Pract. 2009;63(4):568-73.

87. Schäfer W, Kroneman M, Boerma W, van den Berg MJ, Westert GP, Devillé W, et al. The Netherlands: Health system review. Health Systems in Transition. 2010;12(1):1-229.

88. Shaw C. A review of the psychosocial predictors of helpseeking behavior on quality of life in people with urinary incontinence. J Clin Nurs. 2001;10:15-24.

89. Kirby M. Managing stress urinary incontinence - a primary care issue. Int J Clin Pract. 2006;60(2):184-9.

90. Sandvik H, Hunskaar S, Eriksen BC. Management of urinary incontinence in women in general practice: actions taken at the first consultation. Scand J Prim Health Care. 1990;8(1):3-8.

91. Teunissen D, Bosch van den W, Weel van C, Lagro-Janssen ALM. Urinary Incontinence in older people: When and why do general practitioners not follow the guidelines recommendations. Thesis. $\mathrm{Ni}$ jmegen: UMCN; 2006.

92. Shaw C, Atwell C, Wood F, Brittain K, Williams K. A qualitative study of the assessment and treatment of incontinence in primary care. Fam Pract. 2007;24(5):461-7.

93. The Dutch Council for Public Health and Health Care (RVZ). Taakherschikking in de gezondheidszorg. Zoetermeer 2002.

94. Laurant M, Reeves D, Hermens R, Braspenning J, Grol R, Sibbald B. Substitution of doctors by nurses in primary care. Cochrane Database of Systematic Reviews 2004, Issue 4. Art. No.: CD001271. DOI: 10.1002/14651858.CD001271.pub2.

95. Health Council of the Netherlands (Gezondheidsraad). Role redefinition in health care. The Hague: Health Council of the Netherlands, 2008. Report No.: publication no. 2008/25.

96. Kao H, Landefeld C. Improving Primary Care for Older Patients. Arch Intern Med. 2010;170(19):1772-3.

97. NIVEL, the Netherlands Institute for Health Services Research, (The Netherlands), The Technische Universität Berlin, (Germany), The National Board of Health - Danish Centre for Health Technology Assessment, (Denmark), The Academic Medical Centre - University of Amsterdam, (The Netherlands), The London School of Hygiene \& Tropical Medicine, (United Kingdom). Health services research: helping tackle Europe's health care challenges 2011.

98. Laurant M, Harmsen M, Faber M, Wollersheim H, Sibbald B, Grol R. Revision of professional roles and quality improvement: a review of the evidence. London: The Health Foundation. 2010 Contract No.: 6_suppl.

99. Du Moulin MFMT, Hamers JPH, Paulus A, Berendsen C, Halfens R. The role of the nurse in community continence care: a systematic review. Int J Nurs Stud. 2005 5;42(4):479-92.

100.Tugwell P, Bennett KJ, Sackett D, L, Haynes RB. The measurement iterative loop: A framework for the critical appraisal of need, benefits and costs of health interventions. J Chron Dis. 1985;38(4):339-51.

101.Banta D. The development of health technology assessment. Health Policy. 2003;63(2):121-32.

102.Craig P, Dieppe P, Macintyre S, Michie S, Nazareth I, Petticrew M. Developing and evaluating complex interventions: the new Medical Research Council guidance. BMJ. 2008;337(1):a1655-.

103.Lewin S, Glenton C, Oxman AD. Use of qualitative methods alongside randomised controlled trials of complex healthcare interventions: methodological study. BMJ. 2009;339(1):b3496-.

104.Zwarenstein M, Treweek S, Gagnier JJ, Altman DG, Tunis S, Haynes B, et al. Improving the reporting of pragmatic trials: an extension of the CONSORT statement. BMJ. 2008;11(337):a2390. 
Part I

Usual care for patients with urinary incontinence 



\title{
Chapter 2
}

\section{Adherence to professional guidelines for patients with urinary incontinence by general practitioners: \\ A cross-sectional study}

\author{
Pytha Albers-Heitner \\ Bary Berghmans \\ Fred Nieman \\ Toine Lagro-Janssen \\ Ron Winkens
}

Journal of Evaluation in Clinical Practice. 2008;14(5):807-811. 


\section{Abstract}

Background Urinary incontinence (UI) is a common problem, affecting quality of life and leading to high costs. There is much doubt about the use of clinical practice guidelines on UI in primary care.

Objective To assess adherence levels and reasons for (non-)adherence to the Guideline on UI of the Dutch College of General Practitioners.

\section{Methods}

Design, setting and participants. A postal survey among Dutch general practitioners (GPs).

Main outcome measure. Adherence of GPs to the Guideline.

Results We analysed 264 questionnaires. Almost all GPs adhered to the guideline when diagnosing the type of UI. A bladder diary is not often used (35\%). Adherence to therapeutic procedures was only high for mild/moderate stress UI: most GPs (82.6\%) used adequate advice on bladder retraining and pelvic floor muscle training. One out of four GPs agreed that adhering to the guideline is difficult, mainly due to lack of time, staff, diagnostic tools, competencies to provide this care and low motivation of patients.

Conclusions Dutch GPs follow the UI guideline only partially: compliance with diagnostic advices is fairly good; compliance with treatment advices is low. Further research should focus on solutions how to support GPs to tackle major barriers to facilitate the adherence to UI guidelines (substitution of tasks to specialised nurses, reducing the threshold for referral and concentrating expertise in integrated continence care services). 


\section{Introduction}

Urinary incontinence (UI) is a widespread medical problem with a great impact on quality of life and causes high annual costs. However, its prevalence is underreported and under-diagnosed, a fact often not recognised by healthcare providers. Prevalence of 'any UI' in young adult women is $20-30 \%$, increasing to $30-40 \%$ in middle-aged women and to $30-50 \%$ in the elderly. $\mathrm{UI}$ is at least twice as prevalent in women as in men. ${ }^{1,2}$

There are worldwide evidence-based clinical practice guidelines for initial management, assessment and treatment for $\mathrm{UI}$ with lifestyle advices, bladder retraining (BRT) and pelvic floor muscle training (PFMT) for all health professionals dealing with UI. ${ }^{1,3-6}$ All these guidelines contain the same messages and management steps. When patients with UI seek professional help, in many European countries, such as the Netherlands, they will first present to the general practitioner (GP). ${ }^{2,7,8}$ Almost half $(432,100)$ of UI patients in the Netherlands consulting a GP get incontinence pads on prescription to a total amount of costs of roughly 112 million euros each year ( $€ 258$ per patient per year). Pads are the most costly of all healthcare aids. In 2003, after test strips for diabetes and stoma materials, costs for pads showed the highest increase. ${ }^{9,10}$ Because of ageing of the population, a further increase of both incidence and prevalence and costs of $\mathrm{UI}$ is to be expected. ${ }^{3}$ Given the magnitude of this health problem and the high costs, many healthcare experts in this field express their serious doubts about the adequate adherence to dissemination and implementation of UI clinical practice guidelines. ${ }^{11}$ To be able to improve quality of care (non-)adherence of GPs with guidelines on $\mathrm{UI}$ in patient care has to be studied, as well as satisfaction of patients with the quality of this care received from their GP. $^{12,13}$ There are hardly any relevant data on these matters available in the Netherlands nor elsewhere. ${ }^{4,14}$ Therefore we conducted a cross-sectional study (postal survey) in the Netherlands among GPs to assess the level of (non-)adherence of GPs to the Guideline on Urinary Incontinence of the Dutch College of General Practitioners (GLUI). The main study questions were: 'To what extent do GPs adhere to the GLUI? What are reasons for (non-)adherence to components of the GLUI? Is adherence related to specific GP characteristics, patient characteristics or logistic problems?'

\section{Methods}

The primary outcome in the survey is the adherence of GPs to the GLUI. The expected adherence was unknown beforehand. Therefore, to assure a reasonably small standard error on the adherence to be observed and to allow for subgroup analyses on the outcome variable, a number of $300 \mathrm{GPs}$ was initially assumed to be 
needed in data analysis. In earlier surveys GP response to questionnaires was 30$40 \%$, so the number of GPs minimally amounted to $300 \times(1 / 0.35)=857$. Four hundred ninety nine GPs were randomly selected from an alphabetical list of 895 GPs within the south-east of the Netherlands (low urbanisation rate). Another 450 GPs within the west of the Netherlands (high urbanisation rate) were selected from an address list of a regional health insurance company. Representativeness of the sample for region, working hours, gender and years of experience of GPs were cross-checked with published data of Dutch GPs in a National Primary Care survey (NS2). ${ }^{15}$

We used an adapted 'questionnaire on obstructions and needs' ${ }^{16}$ First, a pilot version with 15 respondents was conducted. After modifications, the questionnaire was used in the study. GPs were questioned on their diagnostics and proposed therapy on UI. Moreover, GPs were asked for their view on the GLUI, such as their knowledge, attitudes, perceived problems and needs towards the GLUI, and the care for patients with UI. In the final step, questions on background characteristics of the GPs (gender, age, experience) were added to the list.

A postal questionnaire was sent to 949 GPs between January 2005 and February 2006. To ensure maximum participation the questionnaire was designed for completion within 10 minutes. For 'questions on obstructions and needs', a five-point Likert scale was used with items having answering categories ranging from complete disagreement (score 1 ) to complete agreement (score 5). Definitions of UI conformed to the standards recommended by the International Continence Society $(\text { ICS })^{17}$ except where specially noted.

Means and standard deviations are reported for variables, frequencies and percentages for categorical data. Kolmogorov-Smirnov tests were used to investigate normality of distributions in variables. Bivariate analysis was performed in crosstabulations (with $\chi_{\mathrm{L}}{ }^{2}$ statistics) or by correlations ( $r$, Pearson correlation or $\rho$, Spearman rank correlation coefficient). Independent groups Student's t-tests were performed on normally distributed variables and Mann-Whitney tests on nonnormally distributed variables. Multivariate combinations of categorical data belonging to the same question were analysed by higher-order cross-tabulations to find patterns in response. Multivariate analysis of groups of intended clusters of variables was carried out by factor analysis (principal component analysis) using listwise deletion of missing cases. Cronbach's alpha was used to measure internal consistency of the intended scale(s) found as factors. Final scales were formed by summation over items empirically belonging to the scale divided by the number of nonmissing items. A P-value $<0.05$ was considered statistically significant. 


\section{Results}

Three hundred and sixteen (33\%) questionnaires were returned, of which $52(6 \%)$ returned blank, 264 (28\%) questionnaires could be used for analysis. $158(60 \%)$ male and 54 (21\%) female GPs had completed the questionnaire, 52 (20\%) gender unknown. The sample of 264 GPs was similar to the Dutch GP population as studied in the NS2 on gender, years of working experience and working hours per week. ${ }^{16}$

\section{History taking}

To assess the type of UI almost all 261 (99\%) GPs reported that they probed the patient on ' $\mathrm{UI}$ during abdominal pressure rise', 'urgency to urinate' (98\%) and on 'pain during voiding' (86\%). The remaining issues in history taking are less used. Only $14 \%$ of the GPs considers the role of mobility and/or dexterity in relation to UI (see Table 1).

Table 1 Items reported by GPs during history taking compared to items recommended in the Guideline on Urinary Incontinence of the Dutch College of General Practitioners in percentages and numbers.

\begin{tabular}{ll}
\hline & $\%(\mathrm{n})$ \\
\hline UI with abdominal pressure rise (SUI) & $99(261)$ \\
Urgency in combination with UI (UUI) & $98(258)$ \\
Pain during voiding & $86(228)$ \\
Frequency UI & $78(207)$ \\
Pad use & $71(187)$ \\
Impact UI & $61(162)$ \\
Changed voiding pattern & $58(153)$ \\
Vaginal dryness & $58(152)$ \\
Surgery or diseases related to UI & $57(151)$ \\
Use of medication (diuretics, anticholinergics like antipsychotics or antidepressives) & $52(137)$ \\
Amount of urine loss & $44(117)$ \\
Continuous UI & $42(112)$ \\
Role of impaired mobility or dexterity & $14(38)$ \\
\hline
\end{tabular}

GPs, general practitioners; UI, urinary incontinence; SUI, stress urinary incontinence; UUI, urgency urinary incontinence.

Additional testing (physical examination, urine analysis and bladder diary)

After history taking GPs are advised to examine the lower abdomen and to perform in women a vaginal bimanual palpation and/or a rectal digital palpation, also in men, amongst others for pelvic floor assessment. 
One hundred and sixty-five (65\%) GPs said they always performed a vaginal bimanual palpation and 80 (31\%) GPs only if needed in case of prolapse problems (27), indication of SUI (16), unclear history (11), vaginal atrophy/menopausal problems (4) or unknown (22). Almost half of the GPs (120; 46\%) stated they never performed a rectal palpation. Two hundred and twenty (87\%) GPs reported they always performed a physical examination of the lower abdomen or only after history taking when they suspected residual urine (11), in case of abdominal complaints (12) or in case of an uncertain history (14). Of all GPs, 179 (70\%) always perform urine analysis although the GLUI considers urine analysis not always necessary in case of clear stress urinary incontinence (SUI). One hundred and seventy-one GPs (66\%) asked on no occasion patients to make a bladder diary although advised in the GLUI. ${ }^{5}$

\section{Evaluation of UI}

Almost all GPs (262; 99\%) stated they came to a sufficient reliable preliminary diagnosis in $70 \%$ (median; min. 20, max. $90 \%$ ) of their patients as a result of their diagnostic procedures.

\section{Use of therapeutic procedures}

For mild SUI, one out of five GPs preferred to wait and see $(46 ; 19 \%)$ and half of the responding GPs $(133 ; 55 \%)$ gave oral and/or written advice on BRT and/or PFMT themselves as their first choice therapy (Table 2). The more severe the SUI, the more GPs referred to the physiotherapist (PT) or specialist and the more they prescribed medication. As a second choice for moderate SUI, 106 GPs (45\%) prescribed drugs, $31 \%$ of which were antimuscarinic agents, drugs not indicated for SUI. In case of urgency urinary incontinence (UUI) and mixed urinary incontinence (MUI), GPs did not use oral and/or written advice on BRT and/or PFMT but referred to a specialist and prescribed pads and medication.

Looking at the used 'conservative measures' (instructions on BRT/PFMT), we found that the number of GPs who reported to do so, either as a first or a second choice, was in patients with slight to moderate SUI 83\% (218), severe SUI 50\% (133), UUI 22\% (57) and MUI 15\% (40).

\section{Perceived problems to adhere to the Guideline}

From 145 (56\%) GPs who (strongly) agreed or neither agreed nor disagreed with the item that working according to the GLUI was a problem, the following reasons were identified: lack of staff ( $n=62 ; 43 \%$ ), lack of time for such care ( $n=56 ; 39 \%)$, lack of diagnostic tools ( $n=42 ; 29 \%$ ) and of competencies to give this care ( $n=39 ; 29 \%$ ) and the fact that patients often do not know the advantages of this care $(n=41$; 
28\%). Because of relatively high correlations between the items belonging to 'problems experienced by the GLUI', we performed a factor analysis to test the hypothesis that they could refer to the same problem encountered with the GLUI. One main factor was found (Cronbach's $\alpha$ 0.711) and items were summarised into one uni-dimensional scale 'problems working in accordance with the GLUI' ( $n=113$ ) with a mean score of $3.10(S D=0.77)$. In further analysis, it turned out that especially older, male GPs $(R=-0.38 ; p<0.001$ and $R=-0.55 ; p<0.001)$, who worked more hours per week $(R=0.24 ; p<0.001)$ and who saw more UI patients $(R=0.17$; $p=0.013)$ did experience more 'problems working in accordance with the GLUI'.

Table 2 Self-reported Top-3 frequencies of first and second treatment choices of general practitioners in SUI (mild, moderate, severe), UUI and MUI.

\begin{tabular}{|c|c|c|c|c|}
\hline$N=264$ & First choice & $\mathrm{n}(\%)$ & Second choice & $\mathrm{n}(\%)$ \\
\hline SUI & GP & $133(55)$ & $1=2$ & $120(51)$ \\
\hline \multirow[t]{3}{*}{ mild } & GPO & 46 (19) & PT & $51(22)$ \\
\hline & PT & $36(15)$ & GP & $21(9)$ \\
\hline & & & Spec & $12(5)$ \\
\hline Total & $\mathrm{n}$ & $241(91 ; 100 \%)$ & $\mathrm{n}$ & $231(87.5 ; 100 \%)$ \\
\hline SUI & PT & $72(31)$ & $1=2$ & $64(27)$ \\
\hline \multirow[t]{3}{*}{ moderate } & Spec & $59(26)$ & Med & $106(45)(O=3 ; A=31.3 ; D=10.6)$ \\
\hline & GP & $35(15)$ & GP & $27(11)$ \\
\hline & & & PT & $13(6)$ \\
\hline Total & $\mathrm{n}$ & $230(87.1 ; 100 \%)$ & $\mathrm{n}$ & $236(89.4 ; 100 \%)$ \\
\hline SUI & PT & $59(26)$ & $1=2$ & $38(19)$ \\
\hline \multirow[t]{3}{*}{ severe } & Spec & $48(22)$ & PT & $31(15)$ \\
\hline & Med & $45(20)(O=1.8 ; A=13 ; D=5.4)$ & Pads & $29(14)$ \\
\hline & $\cdots$ & & Spec & $22(11)$ \\
\hline Total & $\mathrm{n}$ & $224(84.4 ; 100 \%)$ & $\mathrm{n}$ & $205(77.7 ; 100 \%)$ \\
\hline \multirow[t]{4}{*}{ UUI } & Spec & $45(22)$ & $1=2$ & $77(38)$ \\
\hline & Pads & $42(21)$ & Pads & $27(13)$ \\
\hline & Med & $35(17)(O=3.4 ; A=7.9 ; D=5.9)$ & Spec & $25(12)$ \\
\hline & & & PT=Med & $20(10)(O=2 ; A=4.5 ; D=3.4)$ \\
\hline Total & $\mathrm{n}$ & $204(77.3 ; 100 \%)$ & $\mathrm{n}$ & $204(77.3 ; 100 \%)$ \\
\hline \multirow[t]{4}{*}{ MUI } & Med & $82(43)(O=3.7 ; A=29.6 ; D=10.1)$ & $1=2$ & $88(50)$ \\
\hline & Spec & $40(21)$ & Med & $27(16)(\mathrm{O}=2.2 ; \mathrm{A}=8.4 ; \mathrm{D}=4.5)$ \\
\hline & Pads & $24(13)$ & Spec & $24(14)$ \\
\hline & & & PT & $16(9)$ \\
\hline Total & $\mathrm{n}$ & $189(72 ; 100 \%)$ & $\mathrm{n}$ & $178(67 ; 100 \%)$ \\
\hline
\end{tabular}

$\mathrm{UI}$, urinary incontinence; SUI, stress urinary incontinence; UUI, urgency urinary incontinence; 1=2: identical first and second choice; GP: GP gave self oral/written advice on bladder retraining and/or pelvic floor muscle training; GPO: advice GP: wait and see; PT: referral physiotherapist diagnostics +/- treatment; SPEC: referral specialist diagnostics +/- treatment; MED: medication; O: estrogens; A: antimuscarinic agents (flavoxaat, oxybutynine, tolterodine; D: different medication); PADs: incontinence pads. 


\section{Discussion}

Our results reveal a partial adherence to the guidelines on $\mathrm{UI}$, thus leaving clear room for improvement.

Before drawing any conclusion on the basis of our findings, the following needs to be considered. The response rate of $28 \%$ is similar to that of other studies amongst GPs in the Netherlands. ${ }^{16}$ It is likely that the non-responders have less knowledge and interest in $\mathrm{UI}$, which is also reported in other studies on $\mathrm{UI}$ in general practice. ${ }^{18,19}$ Consequently, adherence in the total population of GPs may even be lower than adherence reported in this paper.

Almost all GPs adhered to the GLUI with respect to diagnosing the type of UI. However, compliance with the issues on impaired mobility and dexterity was very low. Most GPs probably already know about impaired mobility and dexterity of their patients, but it is not clear whether GPs are aware of the possible relation with UI. There was high compliance with vaginal bimanual digital palpation in women, abdominal check and urine analysis (> 87\%). However, rectal digital palpation was performed much less often. We can only compare these findings on adherence with guidelines with a recent Dutch study on elderly with UI. ${ }^{20}$ In that study adherence to the same diagnostic procedures appeared to be higher. This difference might be explained by the fact that in the other study the participating GPs received a training on the GLUI prior to the study and GPs had to register their actual performance in daily practice. ${ }^{21}$ of all GPs, $66 \%$ never asked their patients to make a bladder diary. The update of the GLUI 2006 stimulates the use a bladder diary. ${ }^{11,19,22}$

Regarding therapeutic procedures it is remarkable that, while for mild SUI GPS seem to follow the guideline adequately, they all of a sudden turn to something irrational as first choice therapy for moderate and severe SUI, UUI and MUI namely the prescription of drugs or referral to the specialist. We could not ask for reasons for this shift, but here we should try to find ways to improve the adherence to the GLUI. One in four GPs agreed that working according to the guideline is a problem. The most important reasons we found for not working in accordance with recommendations of the GLUI (lack of time, staff, diagnostic tools and competencies and low motivation of patients) are in line with studies on patients with $\mathrm{UI}$ in general practice. ${ }^{18,20,23}$ The obstacles mentioned are found in many countries and indicate a potential need for local initiatives for implementation of guidelines to improve quality of care for UI patients both in primary and secondary care. ${ }^{11}$

Considering that adequate UI management by the GP is time consuming, especially in case of more complex problems like severe SUI, UUI and MUI, or concurrent co-morbidities, further research should focus on solutions how to support GPs. One of the solutions could be a substitution of tasks on treatment of UI patients in primary care by nurses specially trained in line with the success of other specialised nurses. ${ }^{24}$ First experiments with this kind of intervention for UI patients seem be to 
be promising, but more research is needed. ${ }^{25}$ It is clear from research that $50 \%$ of people with UI do not seek medical help. ${ }^{26}$ Availability and accessibility of an intervention by specialised nurses might also reduce the threshold for referral.

Moreover, many countries are setting up integrated continence care services to improve the process of referral between primary and secondary care and to offer opportunities to stay up to date with important new developments in the management of bladder problems for all allied healthcare professionals. ${ }^{11,27}$

\section{Conclusions}

Altogether, we conclude that Dutch GPs follow their GLUI only partially: compliance with diagnostic advices is fairly good; compliance with treatment advices is low. Considering that adequate management of UI by GPs is time consuming, especially in case of more complex problems such as severe SUI, UUI and MUI, or concurrent co-morbidities, further research should focus on solutions to tackle major barriers and to introduce facilitators.

\section{Acknowledgements}

This work was supported in part by an unrestricted Grant of Pfizer.

We thank GPs for participating in the survey and Wendy Engering and Huub Verheyden for data entry. 


\section{Practice Recommendations/Take Home message}

There is room for improvement of implementation of guidelines on UI:

- Bladder retraining and pelvic floor muscle training are the first choice therapies in stress-, urgency and mixed UI;

- A bladder diary can help to clarify the type of UI;

- Medications are not first choice treatment for urinary incontinence.

Lack of time and staff of general practitioners seem to be the major barriers to adhere to clinical practice guidelines on urinary incontinence in primary care.

Further research should focus on solutions how to support general practitioners:

- Substitution of tasks to specialised nurses;

- Reducing the barriers for referral;

- Concentrating expertise in integrated continence care services. 


\section{References}

1. Abrams P, Cardozo L, Khoury S, Wein A. Incontinence. $3^{\text {rd }}$ International Consultation on Incontinence. June 26-29, 2004. Monaco: Health Publication Ltd.

2. Norton P, Brubaker L. Urinary incontinence in women. Lancet 2006;367(9504):57-67.

3. Health Council of the Netherlands (Gezondheidsraad). Urine Incontinentie [Urinary Incontinence]. Publication no. 2001/12. The Hague: Health Council of the Netherlands; 2001. Report No.: ISBN: 905549-375-9.

4. Health Insurance Board (CVZ). Urine Incontinentie bij volwassenen [Urinary Incontinence in adults]. Diemen 2003 Contract No.: 2003/147.

5. Lagro-Janssen A, Breedveldt-Boer H, Dongen Jv, Lemain T, Steeneken F, Dijkstra R, et al. Dutch College Guideline on Urinary Incontinence. Huisarts Wet 1995;38(2):71-80.

6. Thüroff J, Abrams P, Andersson K, Artibani W, Chartier-Kastler E, Hampel C, van Kerrebroeck Ph. Guidelines on Urinary Incontinence. European Association of Urology; 2006. Available from: http://www.uroweb.org/fileadmin/user_upload/Guidelines/16\%20Urinary\%20Incontlnence.pdf (accessed 18 April 2008).

7. Monz B HC, Porkess S, Wagg A, Pons ME, Samsioe G, Eliasson T, Chartier-Kastler E, Sykes D, Papanicolaou S. A description of health care provision and access to treatment for women with urinary incontinence in Europe: a five-country comparison. Maturitas 2005;52(Supp 2):S3-12.

8. O'Donnell M, Viktrup L, Hunskaar S. The role of general practitioners in the initial management of women with urinary incontinence in France, Germany, Spain and the UK. Eur J Gen Pract 2007;13(1): 20-6.

9. Health Insurance Board (CVZ). GIP-peilingen. Total cost 2001-2005, hulpmiddelencategorie A: Verzorgingsmiddelen. 2006. Available from: http://www.gipdatabank.nl/index.asp?scherm=lijstTabelsoorten \&infoType=h (accessed 18 April 2008).

10. National Institute for Public Health and the Environment (RIVM). Cost of Illness in the Netherlands. Bilthoven: RIVM; 2006.

11. Kirby M, Artibani W, Cardozo L, Chapple C, Diaz DC, De Ridder D, et al. Overactive bladder: the importance of new guidance. Int J Clin Pract 2006;60(10):1263-1271.

12. Coulter A. Can patients assess the quality of health care? BMJ 2006;333(7557):1-2.

13. Lanier $D$, Roland $M$, Burstin $H$, Knottnerus J. Doctor performance and public accountability. Lancet. 2003;25(362):1404-8.

14. Teunissen D. Urinary incontinence in the elderly in general practice. Thesis. Nijmegen: Radboud University Nijmegen Medical Centre, the Netherlands; 2006.

15. Braspenning J, Schellevis F, Grol R. Second National study on diseases in general practice. Quality of family doctors care. Nijmegen/Utrecht: Center for Quality of Care Research (WOK); 2004.

16. Schellevis F, Westert G, Bakker D de, Groenewegen P. Second National Study in general practice: studyquestion and methods. Nivel; 2004. Report No.: ISBN 90-6905-648-8.

17. Abrams P, Cardozo L, Fall M, Griffits D, Rosier P, Ulmsten U, van Kerrebroeck Ph, Victor A, Wein A. The standardisation of Terminology of Lower Urinary Tract function: Report from the Standardisation Sub-committee of the International Continence Society. Neurourol Urodynam 2002;21:167-178.

18. Lose G, Jacobsen A, Madsen H, Thorsen P, Tibaek S, Johansen B. General practitioners' knowledge of and attitude to assessment and treatment of women with urinary incontinence. A questionnaire among general pratitioners in Denmark. Ugeskr Laeger 2001;163(38):5183-8.

19. Viktrup L, Moller L. The handling of urinary incontinence in Danish general practices after distribution of guidelines and voiding diary reimbursement: an observational study. BMC family practice 2004;5(13).

20. Teunissen D, Bosch van den W, Weel van C, Lagro-Janssen ALM. Urinary incontinence in the elderly: attitudes and experiences of general practitioners. A focus group study. Scand J Prim Health Care 2006;24(1):56-61. 
21. Teunissen D, Bosch van den W, Weel van C, Lagro-Janssen ALM. Urinary Incontinence in older people: When and why do general practitioners not follow the guidelines recommendations. Thesis. Nijmegen: UMCN; 2006.

22. Lagro-Janssen A, Teunissen D, Breedveldt-Boer H, Dongen J v, Lemain T, Steeneken F, Dijkstra R, Wiersma T. Dutch College Guideline on Urinary Incontinence M46. First revision. Huisarts Wet 2006;49(2):501-10.

23. McFall S. Evaluation and treatment of urinary incontinence: report of a physician survey. Arch Fam Med 1997;6:114-119.

24. Vrijhoef $\mathrm{H}$. Is it justifiable to treat chronic patients by nurse specialists? Thesis. Maastricht, The Netherlands: Maastricht University; 2002.

25. Du Moulin MFMT, Hamers JPH, Paulus A, Berendsen C, Halfens R. The role of the nurse in community continence care: a systematic review. Int J Nurs Stud 2005;42(4):479-492.

26. Scottish Intercollegiate Guidelines Network. Guideline 79. Management of urinary incontinence in primary care. A National Guideline. 2004.

27. Kirby M. Managing stress urinary incontinence - a primary care issue. Int J Clin Pract 2006;60(2):184189. 


\title{
Chapter 3
}

\section{How do patients with urinary incontinence perceive care given by their general practitioner?}

A cross-sectional study

\author{
Pytha Albers-Heitner \\ Bary Berghmans \\ Fred Nieman \\ Toine Lagro-Janssen \\ Ron Winkens
}

The International Journal of Clinical Practice. 2008;62(3):508-515. 


\begin{abstract}
Background Urinary incontinence (UI) is a widespread problem, affecting quality of life and leading to high costs, which are mainly caused by incontinence pads. It seems that, despite guidelines, many UI patients get pads from their general practitioner (GP) without adequate diagnostics or treatment.
\end{abstract}

Objectives To assess what further treatment UI patients receive from their GP next to pads and to measure patient satisfaction regarding the current care.

Methods A postal survey among UI patients who started using pads in the past 12 months.

Main outcome Satisfaction with current UI care.

Results We analysed 208 questionnaires. 29.3\% of pad users had no direct contact with their GP for a pad prescription. Of $147 \mathrm{UI}$ patients who visited their GP $50.3 \%$ only got pads. $25 \%$ of patients was not satisfied with information on UI received from their GP, especially younger patients, women, less healthy patients and patients with urgency UI.

Conclusions Dutch UI patients using pads experience suboptimal GP care and management. Factors identified through patient feedback should be used to improve quality of care for UI in primary care. 


\section{Introduction}

Urinary incontinence (UI) is a widespread medical problem with a great impact on quality of life and with high annual costs, which are mainly caused by the use of incontinence pads. However, the prevalence of $\mathrm{UI}$ is under-reported and underdiagnosed, a fact which is often not recognised by healthcare providers. Generally, prevalence of 'any $\mathrm{UI}^{\prime}$ in young adult women amounts to $20-30 \%$, increasing up to $30-40 \%$ in middle-aged females and up to $30-50 \%$ in the elderly. UI prevalence in men, although not investigated to the same extent as for females, appears much lower but increases with age. ${ }^{1-3}$ There are worldwide evidence-based clinical practice guidelines for initial management, assessment and treatment for UI with lifestyle advices, bladder training (BT) and pelvic floor muscle training (PFMT) for all health professionals dealing with UI. In addition to this, medical therapy and other physical therapy adjuncts and devices belong to initial management. ${ }^{1-3}$ By and large all these guideline patterns in primary or in specialised care contain the same messages and initial management steps. When UI patients are seeking professional help, in many European countries, such as the United Kingdom and the Netherlands, they will first present to the general practitioner (GP). ${ }^{3,4}$ Over one million people in the Netherlands suffer from UI. Earlier studies have revealed that one out four of people with UI $(250,000)$ do not seek medical help. From the remaining $750,000 \mathrm{UI}$ patients who do seek medical help $79 \%(590,000)$ get incontinence pads on prescription to a total amount in costs of roughly 160 million euros each year ( $€ 270$ per patient per year). In the Netherlands a first-time prescription by a physician is needed to ensure that pads are paid for by the insurance companies. By their sheer amounts pads are the most expensive of all healthcare aids. Due to an ageing population a further increase of both prevalence and costs of $\mathrm{UI}$ in the near future is to be expected. ${ }^{1}$ It is often hypothesised that pads are necessary and are prescribed next to the recommended/necessary treatment. However, given the magnitude of this health problem and the high costs for incontinence pads, the impression rises that many UI patients get incontinence pads from their GP without adequate diagnostics and treatment. ${ }^{5,6}$ This would imply a very great number of patients with suboptimal care and unnecessary high costs of incontinence pads. Consequently many healthcare experts in this field express their serious doubts about the quality of care for UI. ${ }^{1-3}$

To improve efficacy and acceptability of care it is important to listen carefully to the patients opinion. ${ }^{7}$ Patient feedback surveys are increasingly seen as a key component in monitoring and improving quality of care. ${ }^{8}$ However, there is a limited number of studies evaluating improvement in care in this specific population, hence, availability of data is sparse. ${ }^{9,10}$ Therefore, we conducted a cross-sectional sample survey in the Netherlands among adult UI patients, who recently received incontinence pads. First, we assessed what kind of initial management pad users 
have got from their GP during the past 12 months. Furthermore, we assessed satisfaction of patients with GP care. The main research questions are: "What kind of initial management do UI patients get from their GP and is this management related to patient characteristics? Are pad users satisfied with GP care and is satisfaction related to patient characteristics and type of care received?"

\section{Methods}

Our primary outcome measure is patient satisfaction with care for $\mathrm{UI}$ received from their GP. To avoid recall bias we focused on measuring satisfaction during the 12 months preceding the survey. We assumed that a number of 150-200 UI patients using pads was necessary to accurately and representatively measure the degree of satisfaction with care received. A non-response of $75 \%$ was considered possible, so we contacted approximately 800 pad users to participate in the study. The study was held in 2 Dutch regions. In one region ( $A$, low urbanisation rate) patients were detected from pharmacists data and a private company providing pads. In the other region ( $B$, high urbanisation rate) names and addresses from a regional health insurance company were used. Patients had to be adult and capable to fill in forms. Only patients who had pads prescribed within 12 months were included. Questionnaires were distributed either immediately at delivery of the pads or by post. Representativeness of the sample of UI patients was cross-checked with data from the European Prospective Urinary Incontinence Research (PURE) study. ${ }^{11}$ No questionnaire in the literature was found to answer our specific questions on the satisfaction with care received for UI. Therefore, we developed a pilot questionnaire loosely based on the 'European Project on Patient Evaluation of General Practice Care' (EUROPEP), a standardised and validated instrument to evaluate specific aspects of GP care. $^{12}$

Perceptions of UI care are measured by questions on actual experiences of clinical care (indicators based on Guideline on Urinary Incontinence of the Dutch College of General Practitioners) (GLUI). ${ }^{13}$ Frequency, amount, impact, severity and type of $\mathrm{UI}$ and perceived cause of symptoms of $\mathrm{UI}$ are measured using the International Consultation on Incontinence Questionnaire Short Form (ICIQ-UI SF). The ICIQ-UI SF has been tested and is recommended for use in both clinical and epidemiological studies. ${ }^{14}$ Additionally, patient characteristics were assessed (age, gender and perceived general health with five response options from [very] well to [very] bad). ${ }^{15}$ After a pilot with 15 respondents a revised questionnaire is used in this study. For measuring 'patient satisfaction' statements are used with response measured by a five-point Likert scale with items having answering categories ranging from 'complete disagreement' (score 1) to 'complete agreement' (score 5). Severity of UI is measured using weighted scores on three questions from the ICIQ-UI SF (self- 
reported UI frequency, perceived UI quantity and UI interference with everyday life in the past four weeks) with an overall score theoretically ranging from 0 to 21 . Scores from three items of the self-diagnostic instrument are combined to classify the perceived type of UI as stress (SUI), urgency (UUI) or mixed UI (MUI): 'leaks before you can get to the toilet', 'leaks when you cough or sneeze' and 'leaks when you are physically active/exercising'.

Definitions of UI are conform International Continence Society (ICS) standards. ${ }^{16}$

\section{Statistics}

Means and standard deviations are reported for univariate distributions of variates, frequencies and percentages for categorical data. Kolmogorov-Smirnov tests were used to investigate normality of distributions. Bivariate analysis was performed in cross-tabulations either with $\chi_{\mathrm{L}}{ }^{2}$ statistics or by correlations $(r$, Pearson correlation or $\rho$, Spearman rank correlation coefficient). Independent groups Student's t-tests were performed on normally distributed variates and Mann-Whitney $(M-W)$ tests on non-normally distributed variables. Multivariate distributions of categorical data or items belonging to a domain were analysed by higher-order cross-tabulations to find patterns in response. Logistic regression analysis was done to analyse which patient characteristics were associated with care received. Multivariate analysis of (sets of) items supposed to belong to common domains was performed by principal component analysis using listwise deletion of missing cases. Oblique rotation of factors was used and factor loadings had to be minimally 0.60 or -0.60 . Cronbach's alpha was used to measure the internal consistency of intended scale(s) found as factors. Final scales were formed by summing the scores of the 5-point Likert items belonging to each factor divided by the number of validly scored items. This way the resulting scale scores would also lie within the 1 (fully agree) to 5 (fully disagree) range. Missing data in the numerator of the scale construction were set on zero and patients had to have four out of six valid (non-zero) scores on the items belonging to the scale in the denominator to obtain a valid total scale score. An explorative multivariate (dummy) regression analysis was performed for each of satisfaction scales to analyse if (clinical) patient characteristics such as gender, age, type of UI and severity of UI, did have a statistically significant relationship with them. Type of care given by the GP as reported by patients was also used as a potential predictor in both satisfaction scale models. For this purpose the reported type of care is categorised into four classes: 'only pads', 'advice next to pads', 'referral next to pads and/or advice' and 'medication/pessary next to pads and/or advice'. A p-value of $<0.05$ was considered as statistically significant.

All data were analysed with SPSS-pc, version 12 and 15 (SPSS Inc., Chicago, IL, USA). 


\section{Results}

Between January 2005 and February 2006 questionnaires were handed out to 869 Ul patients using pads on prescription. In region A, 444 (51\%) patients were recruited and 425 (49\%) patients in region B. In total 285 (33\%) questionnaires were returned, 77 (9\%) were blank, thus 208 (24\%) could be further analysed. Major reasons for non-response were 'no/too little UI ( $n=37$ ) or not interested/no time' $(n=14)$.

\section{Patient characteristics}

Respondents were mainly women and they were significantly younger than men ( $p=0.047$ ) (Table 1$)$. Most (86\%) respondents reported having UI at least daily and more than half reported to have moderate to large amounts of UI. The mean ICIQUI SF severity score was 12.6 (SD 3.9). Of the 208 patients, $40 \%$ reported that they presumably suffer from SUI, $14 \%$ from UUI, while the scores from $30 \%$ suggest MUI. Fifteen percent of the patients could not be classified because of lacking responses. More than half of respondents said they had a moderate to (very) poor general health. There is a significant relationship between a poor general health and a higher UI severity sum score (Pearson $r=0.256 ; p=0.001$ ).

Of the 208 patients, $70.7 \%$ remembered to have visited their GP during the past 12 months.

\section{Initial management by the GP}

Half of the patients who consulted their GP reported that they only got pads prescribed (Table 2). Only 16\% percent said they got information, explanation or instructions on making a bladder diary, BT or PFMT. $25 \%$ Reported to be referred to the physiotherapist and/or to the medical specialist. Referral is much higher in patients with a high severity sum score $\left[\chi_{L}^{2}: 7.05\right.$ ( $\left.p=0.008\right)$, OR $=1.169,95 \% \mathrm{Cl} 1.036$ 1.318]. Of all patients $11 \%(n=16)(S U I, 6$; UUI, 4; MUI, 5; other UI, 1$)$ got a prescription for medication of whom $6 \%(n=9)$ as a single treatment. 
Table 1 Demographic and clinical characteristics of the patients in the survey.

\begin{tabular}{|c|c|c|c|}
\hline Patient characteristics & Overall & Females & Males \\
\hline \multirow[t]{2}{*}{ Gender, n (\%) } & $208(100)$ & $183(90.1)$ & $20(9.9)$ \\
\hline & & Missing 5 (2) & \\
\hline \multirow[t]{2}{*}{ Age, mean \pm SD } & $63.6(13.1)$ & $62.9(13.0)$ & $68.8(13.7)^{*}$ \\
\hline & missing 16 (7.7) & missing 19 (9.1) & \\
\hline \multicolumn{4}{|l|}{ UI, frequency, n (\%) } \\
\hline$\leq 1 / w k$ & $12(5.8)$ & $10(4.9)$ & $2(1.0)$ \\
\hline $2 / 3 / w k$ & $18(8.7)$ & $15(7.4)$ & $3(1.5)$ \\
\hline \multirow[t]{2}{*}{$>7 / w k$} & $178(85.6)$ & $158(77.8)$ & $15(7.4)$ \\
\hline & & missing 5 (2.4) & \\
\hline \multicolumn{4}{|l|}{ UI, amount, n (\%) } \\
\hline small & $86(42.0)$ & $77(38.3)$ & $8(4)$ \\
\hline moderate & $97(47.3)$ & $87(43.3)$ & $7(3.5)$ \\
\hline \multirow[t]{2}{*}{ large } & $22(10.7)$ & $17(8.5)$ & $5(2.5)$ \\
\hline & missing 3 (1.4) & missing 7 (3.4) & \\
\hline UI, impact on daily life, & $5.6(2.7)$ & $5.5(2.7)$ & $6.6(2.9)$ \\
\hline mean \pm SD (scale $0-10)$ & missing 28 (13.5) & missing 31 (14.9) & \\
\hline \multirow{2}{*}{$\begin{array}{l}\text { ICIQ-UI SF† severity sum score, } \\
\text { mean } \pm \text { SD (score } 0-21)\end{array}$} & $12.6(3.9)$ & $12.5(3.8)$ & $13.8(4.9)$ \\
\hline & missing 30 (14.4) & missing 33 (15.9) & \\
\hline \multicolumn{4}{|l|}{ Ul type $^{+}, \mathrm{n}(\%)$} \\
\hline SUI & $84(40.4)$ & 75 (36.9) & $5(2.5)$ \\
\hline UUI & 29 (13.9) & $25(12.3)$ & $4(2)$ \\
\hline MUI & $63(30.3)$ & $63(31)$ & 0 \\
\hline \multirow[t]{2}{*}{ Other/unknown } & $32(15.4)$ & $20(9.9)$ & $11(5.4)$ \\
\hline & & missing 5 (2.4) & \\
\hline \multicolumn{4}{|l|}{ General health, n (\%) } \\
\hline (very) good & 81 (39.5) & $72(36)$ & $7(3.5)$ \\
\hline moderate & $89(43.4)$ & $79(39.5)$ & $8(4)$ \\
\hline \multirow[t]{2}{*}{ (very) bad } & 35 (17.1) & $29(14.5)$ & $5(2.5)$ \\
\hline & missing 3 (1.4) & missing 8 (3.8) & \\
\hline \multirow[t]{2}{*}{ Visited the GP in the past 12 months, $\mathrm{n}(\%)$} & $147(70.7)$ & $130(90.3)$ & $14(9.7)$ \\
\hline & & missing 3 (2) & \\
\hline
\end{tabular}

UI, urinary incontinence; ICIQ-UI SF, International Consultation on Incontinence Questionnaire Short Form; SUI, stress urinary incontinence; UUI, urgency urinary incontinence; MUI, mixed urinary incontinence; GP, general practitioner. Because of rounding off the (valid) \% differs from $100 \%$ * ${ }^{\text {Student's t- }}$ test, $\mathrm{p}=0.047 .{ }^{+}$Conform ICIQ-UI Short Form. ${ }^{14}$ 
Table 2 Received type of care from GP as reported by UI pad users who visited their GP at least once during the past year.

\begin{tabular}{lcc}
\hline Received type of care reported by UI patients & Freq & $\%$ \\
& 147 & 100 \\
\hline Only pads & 740.3 \\
Pads plus advice on BT or PFMT from GP (verbally, during vaginal examination only & 23 & 15.6 \\
and/or by means of a bladder diary) & 16 \\
Pads plus referral to physiotherapist (some combined with advice/information from & 10.9 \\
GP) & 13 \\
$\begin{array}{l}\text { Pads plus referral to medical specialist (some combined with advice/information from } \\
\text { GP) }\end{array}$ & 8.8 \\
Pads plus referral both to PT and to medical specialist (some combined with ad- & 7 & 4.8 \\
vice/information from GP) & 10 \\
Pads plus only medication (9) and pessary (1) & 6.8 \\
Pads plus other (unknown) & 4.7 \\
\hline
\end{tabular}

GP, general practitioner; BT, Bladder Training; PFMT, Pelvic Floor Muscle Training; PT, physiotherapist. Because of rounding off the (valid) \% differs from $100 \%$.

\section{Patient satisfaction}

In principal component analysis on items measuring satisfaction with the amount and quality of GP care two factors were found (Table 3). The first can be named 'personal attention received with regard to $\mathrm{UI}^{\prime}$, the second can be called 'information and advice received with regard to UI'. Reliability of both intended scales was sufficient (Cronbach's $\alpha=0.96$ and $\alpha=0.97$, respectively), and no items had to be removed to enhance reliability even further. Overall scale mean for the evaluation of 'personal attention' is 4.31 (SD 1.03), so, the percentage of patients agreeing with these positively stated items is approximately $100 \% * 4.31 / 5.00=86 \%$. Overall scale mean for the evaluation of 'advice and information' appears to be somewhat lower (3.69), so, the percentage of patients agreeing with these positively stated items is approximately $100 \% * 3.69 / 5.00=74 \%$. 
Table 3 Satisfaction of patients with 'personal attention' and 'information and advice' on UI given by the GP during consultation on found correlations ( $n=147)$.

\begin{tabular}{|c|c|c|c|c|}
\hline $\begin{array}{l}\text { Personal attention: } \\
\text { "Regarding UI: }\end{array}$ & $\begin{array}{l}\text { Response } \\
\mathrm{n}(\%)\end{array}$ & $\begin{array}{l}\text { Scale mean (SD) } \\
1=\text { do not agree at all } \\
5=\text { completely agree }\end{array}$ & \multicolumn{2}{|c|}{$\begin{array}{l}\text { Factor loadings in } \\
\text { Obliquely rotated } \\
\text { Principal Component } \\
\text { Analysis }\end{array}$} \\
\hline ...GP offered help quickly" & $110(75)$ & $4.27(1.6)$ & 0.980 & 0.163 \\
\hline ...GP offered good help" & $98(67)$ & $4.26(1.2)$ & 0.915 & 0.027 \\
\hline ...GP was interested in my case" & $114(78)$ & $4.33(1.1)$ & 0.896 & 0.064 \\
\hline ...GP listened well" & $114(78)$ & $4.35(1.1)$ & 0.862 & 0.070 \\
\hline ...GP had enough time for me" & $126(86)$ & $4.21(1.2)$ & 0.796 & 0.178 \\
\hline $\begin{array}{l}\text {...GP advised me so I felt well enough to } \\
\text { do my regular daily activities" }\end{array}$ & $98(67)$ & $4.11(1.3)$ & 0.770 & 0.155 \\
\hline Overall scale attention & $\begin{array}{l}115(78.2) \\
\text { missing } 32\end{array}$ & $\begin{array}{l}4.31(1.03) \\
1.7)\end{array}$ & Cronba & $=0.96$ \\
\hline \multicolumn{5}{|l|}{$\begin{array}{l}\text { Advice and information: } \\
\text { "Regarding UI: }\end{array}$} \\
\hline $\begin{array}{l}\text {...GP explained the purpose of tests and } \\
\text { treatment" }\end{array}$ & $94(64)$ & $3.41(1.5)$ & 0.145 & 1.029 \\
\hline $\begin{array}{l}\text {...GP helped me to see the importance of } \\
\text { following his/her advice" }\end{array}$ & $94(64)$ & $3.66(1.4)$ & 0.014 & 0.948 \\
\hline $\begin{array}{l}\text {...GP prepared me for what to expect from } \\
\text { treatment or referral" }\end{array}$ & $63(43)$ & $3.51(1.4)$ & 0.013 & 0.965 \\
\hline ...GP told me what I wanted to know on UI & $97(66)$ & $3.79(1.4)$ & 0.072 & 0.871 \\
\hline $\begin{array}{l}\text {...GP did involve me in decisions about } \\
\text { medical care" }\end{array}$ & $96(65)$ & $3.89(1.4)$ & 0.208 & 0.709 \\
\hline $\begin{array}{l}\text {...GP offered suggestions for prevention } \\
\text { (e.g. advice on BT, PFMT and lifestyle)" }\end{array}$ & $96(65)$ & $3.75(1.4)$ & 0.272 & 0.631 \\
\hline Overall scale information & $\begin{array}{l}92(62.6) \\
\text { missing } 55\end{array}$ & $\begin{array}{l}3.69(1.27) \\
37.4)\end{array}$ & Cronba & $\alpha=0.97$ \\
\hline
\end{tabular}

UI, urinary incontinence; GP, general practitioner; BT, Bladder Training; PFMT, Pelvic Floor Muscle Training.

Using the scale 'evaluation of the personal attention' the GP has given to the UI problem as a dependent variable the final (dummy) regression model includes four statistically significant predictors: age, general health perception, type of $\mathrm{UI}$ and the type of care received (Table 4). Younger people, the self-assessed less healthy people and patients with UUI are less satisfied with the attention they received from the GP ( $p=0.016, p=0.041$ and $p=0.007$ respectively). Patients who have been referred are more satisfied with the attention from the GP $(p=0.013)$. Predictors 
tend to strengthen each other within the regression model, e.g. having UUI compared to SUI is significantly correlated with higher age $(r=0.32, p<0.001)$, and UUI patients seem referred more often than SUI patients $(r=0.16, p=0.040)$.

Using the scale 'evaluation of the information and advice' the GP has given concerning the UI problem as a dependent variable the final (dummy) regression model includes five statistically significant predictors: patient gender and age, general health perception, type of $\mathrm{UI}$ and the type of care received (Table 5). Women, younger people and the less healthy people are less satisfied with the advice and information they received (respectively $p=0.023, p=0.006$ and $p=0.049$ ). Patients who have been referred and patients with SUI are more satisfied about advice and information from the GP ( $p=0.001$ and 0.003 respectively).

Table 4 Final model (dummy)regression analysis results using the patient's 'evaluation of the personal attention' as a 'dependent' variable. Variance explained .220 ; listwise deletion of missing cases $(n=87)$.

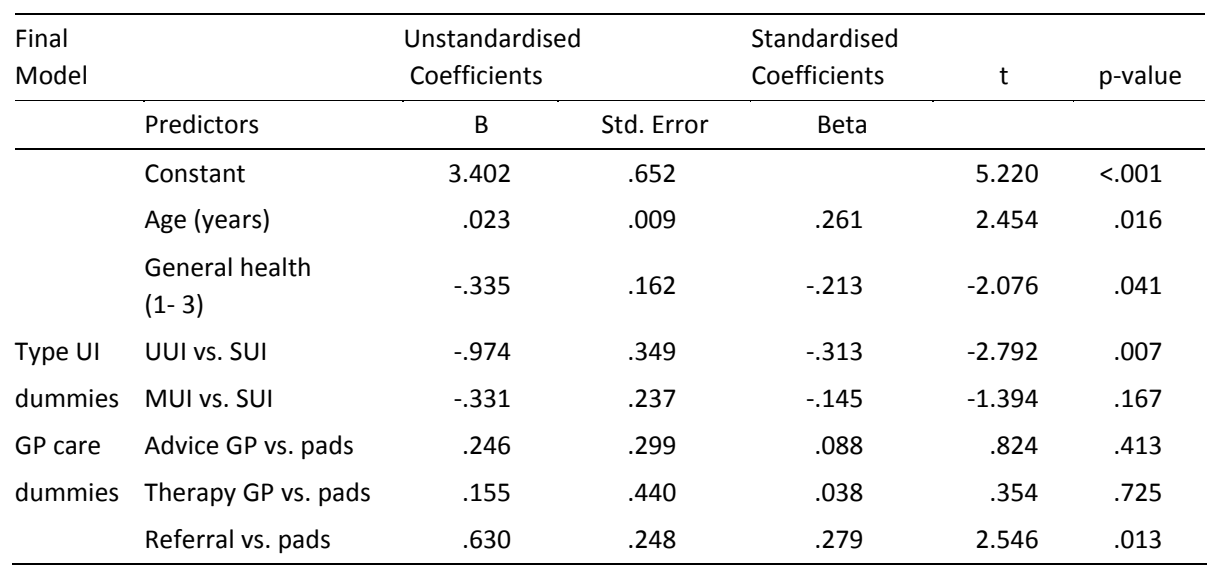

UI, urinary incontinence; SUI, stress urinary incontinence; UUI, urgency urinary incontinence; MUI, mixed urinary incontinence; GP, general practitioner; PT, physiotherapist

Scale values of 'personal attention' lie between $1=$ low evaluation and $5=$ high evaluation. The three 'GP care' dummy variables (having 0-1 scores) contrast GP advice, - therapy ánd referral to other caregivers (PT, specialist) with only pads (zero-score control group); the two 'type of UI' dummy variables (0-1) contrast 'UUI' and 'MUI' with 'SUI' (zero-score control group). General health evaluation is a trichotomy with categories 1 = (very) good, 2 = moderate, 3 = (very) bad. A positive coefficient indicates a higher evaluation of the 'personal attention', a negative a lower one. 
Table 5 Final model (dummy)regression analysis results using the patient's evaluation of the 'information and advice' as a 'dependent' variable. Variance explained .376; listwise deletion of missing cases $(n=70)$.

\begin{tabular}{|c|c|c|c|c|c|c|}
\hline \multirow[t]{2}{*}{$\begin{array}{l}\text { Final } \\
\text { Model }\end{array}$} & \multirow[b]{2}{*}{ Predictors } & \multicolumn{2}{|c|}{$\begin{array}{l}\text { Unstandardised } \\
\text { Coefficients }\end{array}$} & \multirow{2}{*}{$\begin{array}{c}\begin{array}{l}\text { Standardised } \\
\text { Coefficients }\end{array} \\
\text { Beta }\end{array}$} & \multirow[t]{2}{*}{$\mathrm{t}$} & \multirow[t]{2}{*}{$p$-value } \\
\hline & & B & Std. Error & & & \\
\hline & Constant & 2.017 & .799 & & 2.525 & .014 \\
\hline & Age(years) & .032 & .011 & .314 & 2.865 & .006 \\
\hline & Gender(ઠ̋vs.우) & 1.297 & .554 & .261 & 2.341 & .023 \\
\hline & General health (1-3) & -.407 & .202 & -.212 & -2.012 & .049 \\
\hline \multirow[t]{3}{*}{ GP care } & Advice GP vs. pads & .671 & .359 & .203 & 1.867 & .067 \\
\hline & Therapy GP vs. pads & .365 & .675 & .058 & .541 & .591 \\
\hline & Referral vs. pads & 1.145 & .330 & .397 & 3.473 & .001 \\
\hline \multirow[t]{2}{*}{ Type UI } & UUI vs. SUI & -1.473 & .479 & -.384 & -3.075 & .003 \\
\hline & MUI vs. SUI & -.102 & .301 & -.037 & -.340 & .735 \\
\hline
\end{tabular}

Legends and for dummies description, see Table 4.

Scale values of 'information and advice' lie between $1=$ low evaluation and $5=$ high evaluation. Gender is categorised as $\sigma^{\top}=2$ and 우 $=1$. A positive coefficient indicates a higher evaluation of the 'information and advice', a negative a lower one.

\section{Discussion}

\section{Main results}

To our knowledge, this is the first study, where UI patients, who recently got their first prescription for pads, have reported in detail on their experiences with GP care. First, our study reveals that one out of three UI patients had admittedly no direct contact with their GP for their pad prescription. This obviously indicates that pads are prescribed through a practice nurse/GP assistant as a delegated task. It also surmises that, once pads are prescribed, effects of advices on treatments like BT and/or PFMT and pad use are not monitored by the GP over time as advised in guidelines. $^{13}$

Second, over half of the UI patients who visited their GP said they only got pads. It seems obvious that these patients either received no adequate diagnostics and treatment aimed on cure or prevention as recommended as initial management in guidelines or they could not remember any attempts of this from their GP. ${ }^{1-3}$ These findings confirm the self-reported results on GP management in the same parts of the Netherlands at the same period in a separate study. ${ }^{6}$ Our study also supports the impression that the compliance of GPs with the diagnostic advices might be doubtful. The other half of UI patients either got 'treatment' by their GP or were referred to the PT or the medical specialist. This level of 'any conservative treatment besides pads' is higher than that found in the PURE study within Germany, 
Spain or UK/Ireland (respectively $32 \%, 20 \%$ and $45 \%) .{ }^{17}$ Our referral rate $(25 \%)$ and the prescription of medications for UI (11\%) is higher than Dutch data of new patients with $\mathrm{UI}$ in primary care of $12 \%$ and $9.8 \%$ respectively. [Unpublished data from the Second Dutch National Survey 28-08-2007] This might be explained by the fact that we only selected pad users who might have more severe and complex UI. Prescription of medication is very low compared to data of the PURE study from Germany, Spain and UK/Ireland (41\%, 25\% and 39\%). Medication, in addition to lifestyle advices, BT and PFMT, especially in patients with UUI and MUI for the UUI component, can be used to optimise treatment effect. Nevertheless, Dutch GPs appear to be reluctant in the prescription of medication, which is in agreement with guidelines.

Third and finally, we found that one out of four UI patients is not satisfied with the information and advice on UI received from their GP, particularly in younger patients, women, self-proclaimed less healthy patients and patients with UUI. The satisfaction rate on information and advice is low compared to primary care studies where in general $80-90 \%$ of patients is satisfied with issues on information received during consultation. ${ }^{18}$ One explanation might be that half of the respondents only got pads from their GP and in general being given more information tends to correlate with higher satisfaction levels.

\section{Strengths and limitations of the study}

Before drawing any conclusions on the basis of our findings the following needs to be considered.

The 183 women in our sample appear to be similar on age and urbanisation with the 778 Dutch women in the PURE study. ${ }^{11}$

Although our data on distribution for type of UI may be somewhat biased, as we only could classify 163 women because of missing scores on items needed to classify patients, distribution is rather consistent with those from epidemiological literature. ${ }^{2,3}$ Patients in our study reported more frequent and more severe UI than the population in the PURE study. This seems logical as the PURE study included all UI patients in primary care and not only pad users. ${ }^{11}$ We were especially interested in patients who were still using pads or were prescribed pads and generally this would indicate a group that is still incontinent and likely did not receive adequate therapy. This is an inherent bias of the study design.

Increase of prevalence of $\mathrm{UI}$ in men over 80 might explain the few male respondents in our study. ${ }^{1}$

Most studies have a large fraction of other/unclassified types $\mathrm{UI}$ in men. ${ }^{2}$

One of the potential limitations of this study is that it relies on self-reported retrospective data instead of medical records. Consequently, there is a chance of recall bias where patients report inaccurate or incomplete information on care provided 
by the GP during the past 12 months prior to this study. Next to this, we did not ask patients for reasons why they did or did not get their therapies in this study. It is possible that patients refused any prospect of a referral or a prescription for medication and only wanted a pad. In two Dutch studies GPs mention that patient motivation played a role in working according to guidelines on UI.,

Moreover, the data might be biased by missing respondents, whose reasons not to participate are mostly various. ${ }^{8}$ As the study is cross-sectional, we cannot test for causal relationships, but we can only examine statistical associations.

Finally, we analysed 208 questionnaires which means a crude response rate of $24 \%$. This seems low compared to response rates for patients' surveys with a mean response rate of $60 \%$ (SD 21\%). ${ }^{19}$ One explanation might be that besides an explanatory letter with standard information which accompanied the questionnaire, no reminders or any incentives were used. Nonetheless, the respondents showed to be representative (age, gender, urbanisation, type and severity of UI) for Dutch patients in primary care who got pads prescribed. This way the possibility that results from respondents differ in a meaningful way from non-respondents, thus biasing results, seems not plausible. ${ }^{19}$

\section{Practical consequences}

Our findings confirm the impression that too many $\mathrm{UI}$ patients only receive pads without adequate diagnostics and treatment by their GP as recommended in clinical practice guidelines on UI. ${ }^{13}$ A large number of $\mathrm{UI}$ patients may receive suboptimal care and because of life-long use of pads this will lead to often unnecessary high costs of incontinence pads since no cure or improvement is aimed for. Our findings confirm results of reviews on the use of $\mathrm{UI}$ guidelines in several European countries. There is a potential need for local initiatives that take into account patient perceptions to implement guidelines and to improve quality of care for UI patients. ${ }^{6,8,20,21}$ In line with other chronic diseases, adequate UI management by the GP requires more time than GPs have available for patient care overall. This is especially true in case of more complex problems such as severe SUI, UUI and MUI, or concurrent comorbidities. Therefore, further research should focus on solutions how to support GPs to meet recommended standards for quality of care for UI. One of the solutions might be multidisciplinary guidelines as developed by a team of Dutch GPs and physiotherapists e.g. "National Multidisciplinary Agreement" (LESA). ${ }^{22}$ Next to this, educating the physicians, with the inclusion of guidelines in the medical syllabus, at an early stage in their training, may help raise awareness of valuable tools and could also make a worthwhile impact. ${ }^{21}$ Another solution might be alternative methods of service delivery, such as a substitution of tasks on treatment of UI patients in primary care by continence nurses, specially trained in line with the success of other specialised nurses. First experiments with this kind of intervention for UI patients 
seem be to be promising, but more research is needed. ${ }^{23}$ Earlier studies have revealed that one out four of people with $\mathrm{UI}$ do not seek medical help. ${ }^{13}$ Availability and accessibility of an intervention by specialised continence nurses might also lower the threshold for patients to seek help.

Moreover, many countries are setting up integrated continence care services to improve the process of referral between primary and secondary care and to offer opportunities to stay up to date with important new developments in the management of bladder problems for all allied healthcare professionals. ${ }^{20,21,24}$

\section{Conclusion}

Dutch UI patients using pads on prescription experience suboptimal GP care and management. Considering that adequate GP UI management is time consuming, especially in case of more complex problems such as severe SUI, UUI and MUI, or concurrent co-morbidities, further research should focus on effective primary care interventions to support GPs and on solutions to tackle major barriers and to introduce facilitators. Factors identified through patient feedback should be used to improve quality of care for UI in primary care.

\section{Acknowledgements}

This work was supported in part by an unrestricted Grant of Pfizer. The authors thank pharmacists, MediReva V.O.F., Maastricht and Azivo, The Hague, for support and distributing the questionnaires. We thank Wendy Engering and Huub Verheijden for data entry and patients for participating in the survey. 


\section{What is already known about this topic?}

- Urinary incontinence (UI) is a widespread problem with great impact on quality of life and with high annual costs for patients, health insurance companies and society in general;

- Worldwide evidence-based clinical practice guidelines recommend lifestyle advices, bladder training and pelvic floor muscle training as the first step in initial management of stress-, urgency and mixed UI;

- Many healthcare experts in this field express their serious doubts about the quality of care for UI.

\section{What does this article add?}

- Dutch UI patients using pads (care) on prescription ${ }^{1}$ experience suboptimal general practitioner (GP) management (cure and care);

- Patients with UI want more information and education about their incontinence than currently provided;

- This article provides necessary patient feedback for improvement of GP management of UI.

\footnotetext{
${ }^{1}$ In the Netherlands a first-time physicians prescription is needed to ensure that pads are paid for by the insurance companies
} 


\section{References}

1. Teunissen TAM, Bosch van den WJHM, Hoogen van den HJM, Lagro-Janssen ALM. Prevalence of urinary, fecal and double incontinence in the elderly living at home. Int Urogynecol J. 2004;15(1):10-3.

2. Abrams P, Cardozo L, Khoury S, Wein A. Incontinence. 3rd International Consultation. Monaco: Health Publication Ltd. 2005.

3. Norton P, Brubaker L. Urinary incontinence in women. Lancet. 2006;367:57-67.

4. O'Donnell M, Viktrup L, Hunskaar S. The role of general practitioners in the initial management of women with urinary incontinence in France, Germany, Spain and the UK. Eur J Gen Pract. 2007;13(1):20-6.

5. Teunissen TAM, Bosch van den W, Weel van C, Lagro-Janssen ALM. Urinary incontinence in the elderly: attitudes and experiences of general practitioners. A focus group study. Scand J Prim Health Care. 2006;24(1):56-61.

6. Albers-Heitner P, Berghmans B, Nieman F et al. Adherence to professional guidelines for patients with urinary incontinence by family doctors. A cross-sectional study. J Eval Clin Pract. 2008;14(5):807-11.

7. Hu T-W, Wagner TH. Health-related consequences of overactive bladder: an economic perspective. BJU Int. 2005;96:43-5.

8. Coulter A. Can patients assess the quality of health care? BMJ. 2006;333:1-2.

9. Monz B, Pons M, Hampel C et al. Patient-reported impact of urinary incontinence-Results from treatment seeking women in 14 European countries. Maturitas. 2005;30(52):suppl 2: 24-34.

10. Teunissen TAM, Weel van C, Lagro-Janssen ALM. Urinary incontinence in older people living in the community examining helpseeking behaviour. Br J Gen Pract. 2005;55:776-82.

11. Sykes D, Castro R, Pons ME et al. Characteristics of female outpatients with urinary incontinence participating in a 6-month observational study in 14 European countries. Maturitas.2005; 30(52):suppl2:13-23.

12. Wensing M, Mainz J, Grol R. Standardised instrument for patient evaluations of general practice care in Europe. Eur J Gen Pract. 2000;6:82-7.

13. Lagro-Janssen ALM, Teunissen D, Breedveldt-Boer HP et al. NHG-Standaard Incontinentie voor Urine M46 Eerste herziening (Dutch College Guideline on Urinary Incontinence. First revision). Nederlands Tijdschrift voor Urologie. 2006;49(2):501-10.

14. Avery K, Donovan J, Peters T et al. ICl-Q: a brief and robust measure for evaluating the symptoms and impact of urinary incontinence. Neurourol Urodynam. 2004;23(4):322-30.

15. Burstrom B, Fredlund P. Self rated health: Is it as good a predictor of subsequent mortality among adults in lower as well as in higher social classes? J Epidemiol Community Health. 2001;55(11):836-40.

16. Abrams P, Cardozo L, Fall M et al. The standardisation of Terminology of Lower Urinary Tract function: Report from the Standardisation Sub-committee of the International Continence Society. Neurourol Urodynam. 2002;21:167-78.

17. Papanicolaou S, Pons ME, Hampel C et al. Medical resource utilisation and cost of care for women seeking treatment for urinary incontinence in an outpatient setting. Examples from three countries participating in the PURE study. Maturitas. 2005;30(52):suppl 2:35-47.

18. Grol R, Wensing M, Mainz J et al. Patients in Europe evaluate general practice care: an international comparison. Br J Gen Pract. 2000;50(460):882-7.

19. Asch D, Jedrziewski M, Christakis N. Response rates to mail surveys published in medical journals. J Clin Epidemiol. 1997;50(10):1129-36.

20. Kirby M. Managing stress urinary incontinence - a primary care issue. Int J Clin Pract. 2006;60(2):184-9.

21. Kirby M, Artibani W, Cardozo L et al. Overactive bladder: the importance of new guidance. Int J Clin Pract. 2006;60(10):1263-71.

22. Faber E, Custers JWH, Berghmans LCM et al. Landelijke Eerstelijns Samenwerkings Afspraak Incontinentie voor Urine [National First Line Multidisciplinary Agreement] 'LESA'. Huisarts Wet. 2007;50(5): S9-12. 
23. Du Moulin MFMT, Hamers JPH, Paulus A et al. The role of the nurse in community continence care: a systematic review. Int J Nurs Stud. 2005 2005/5;42(4):479-92.

24. Monz B, Hampel C, Porkess S et al. A description of health care provision and access to treatment for women with urinary incontinence in Europe: a five-country comparison. Maturitas. 2005; 16(52):suppl 2:3-12. 



\section{Part II}

Involvement of nurse specialists in primary care for urinary incontinence 



\section{Chapter 4}

\section{The effects of involving a nurse practitioner in primary care for adult patients with urinary}

\section{incontinence:}

The PromoCon study (Promoting Continence)

Study protocol ISRCTN62722772

Pytha Albers-Heitner

Bary Berghmans

Manuela Joore

Toine Lagro-Janssen

Johan Severens

Fred Nieman

Ron Winkens

BMC Health Services Research. 2008(8):84. 


\begin{abstract}
Background Urinary incontinence (UI) affects approximately $5 \%(800,000)$ of the Dutch population. Guidelines recommend pelvic floor muscle/bladder training for most patients. Unfortunately, general practitioners (GPs) use this training only incidentally, but prescribe incontinence pads. Over $50 \%$ of patients get such pads, costing $€ 160$ million each year. Due to ageing of the population a further increase of expenses is expected. Several national reports recommend to involve nurse specialists to support general practitioners and improve patient care. The main objective of our study is to investigate the effectiveness and cost-effectiveness of involving nurse specialists in primary care for UI. This paper describes the study protocol.

Methods/Study design In a pragmatic prospective multicentre two-armed randomised controlled trial in the Netherlands the availability and involvement for the GPs of a nurse specialist will be compared with care-as-usual. All consecutive patients consulting their GP within one year for UI and patients already diagnosed with UI are eligible. Included patients will be followed for 12 months.

Primary outcome is severity of UI (measured with the International Consultation on Incontinence Questionnaire Short Form [ICIQ-UI SF]). Based on ICIQ-UI SF outcome data the number of patients needed to include is 350 . For the economic evaluation quality of life and costs will be measured alongside the clinical trial. For the longer term extrapolation of the economic evaluation a Markov modelling approach will be used.
\end{abstract}

Discussion / Conclusion This is, to our knowledge, the first trial on care for patients with $\mathrm{UI}$ in primary care that includes a full economic evaluation and costeffectiveness modelling exercise from the societal perspective. If this intervention proves to be effective and cost-effective, implementation of this intervention is considered and anticipated. 


\section{Background}

\section{Problem definition}

\section{The disease}

Approximately $5 \%(800,000$ people) of the Dutch population suffer from urinary incontinence (UI). ${ }^{1}$ This concerns predominantly women, and prevalence increases with age. ${ }^{2}$ Among patients aged over 75 , the prevalence rises up to $30 \%$. UI is infamous for its impact on general well-being and social activities. ${ }^{3,4}$ If not treated, $\mathrm{UI}$ is a chronic, not self-limiting disorder with a strong tendency to worsen over time. It is one of the most important reasons for institutionalisation of elderly people in nursing homes.

\section{The healthcare problem}

In the Netherlands, annually $€ 160$ million is spent on incontinence pads. ${ }^{1}$ Considering the ageing of the population a further increase in these expenditures is expected. ${ }^{5-7}$ Guidelines indicate that for most patients with UI pelvic floor muscle and/or bladder training is the best non-invasive treatment to solve the problem, rather than just compensate the urine loss. ${ }^{2,5,6,8,9}$ To date, despite guidelines, training is used only incidentally by general practitioners (GPs), probably because it is too time consuming. ${ }^{5,6,8,10-12}$ Most GPs choose an easier, but non-curative and ultimately far more expensive, alternative: prescribing incontinence pads. More than $50 \%$ of patients get incontinence pads, especially the elderly. ${ }^{1,12}$

\section{Usual care}

For the vast majority of health problems in the Dutch population, the GP is the initial person to contact and is also the gatekeeper for specialist care. This also applies for UI. Most patients ( $\pm 96 \%$ ) with UI are kept within primary care. ${ }^{9,13}$ Only few patients (total $<5 \%$ ) are referred to a specialist (in most cases a urologist or gynaecologist) or a physiotherapist. ${ }^{5,13}$ National guidelines on UI are available for primary care. ${ }^{9,14}$ These guidelines are followed only incidentally, irrespective of the type of guideline or clinical problem involved. ${ }^{10-12,15} \mathrm{~A}$ major reason why the guidelines are not used, may be because consultations take more time when following guidelines. $^{5,16}$

\section{Motivation and relevance for the chosen intervention}

In recent years, there is a growing emphasis in the Netherlands to involve nurse specialists in general practice with positive findings for chronic disorders. $5,6,17,18$ Nurse specialists have specific expertise, and are capable of spending more time on motivating patients. Incorporating nurse specialists may offer a solution to the inadequate care for UI with inappropriate prescribing of incontinence pads. After 
special training, and using their specific expertise on UI, they are well equipped to support the GP after the initial problem definition by the GP. Their acceptance by patients and GPs, feasibility and usefulness in management, and effectiveness in treating UI have been reported. ${ }^{8,19-23}$ However, to our knowledge, studies with a full economic analysis and cost-effectiveness modelling exercise from the societal perspective of involving nurse specialists for UI are not available.

\section{Objective}

The main objective is to study whether the availability and involvement of a nurse specialist in a new role as a substitute for the GP in the management of UI in general practice leads to more efficient care for adult UI patients. We envision that the effects of the intervention will be such that care will improve, hence more patients can be treated effectively and therefore will use less incontinence pads, which leads to a reduction in costs.

The following research questions were formulated:

1. Does the availability and the involvement of a nurse specialist as a substitute for the GP for adult persons with UI lead to a reduction in the severity of UI compared to care-as-usual by the GP?

2. Does the involvement of such a nurse specialist lead to a better quality of life of the patients?

3. Is the involvement of such a nurse specialist cost-effective compared to care-asusual by the GP?

4 Does the involvement of a nurse specialist on UI lead to a better satisfaction of patients, GPs and other healthcare providers?

5. To what extent do GPs use the nurse specialist?

6. What are reasons for the GPs for (not) using the nurse specialist? Does the availability of the nurse specialist change the perception of GPs about the treatment via the nurse specialist?

\section{Methods / Study design}

This pragmatic prospective multicentre two-armed randomised controlled trial is now conducted among patients with $\mathrm{UI}$ in general practices in four regions in the Netherlands. Initially, the trial was planned to be conducted in at least two regions (Maastricht and Nijmegen). During the preparation of the trial expert key persons on $\mathrm{UI}$ in the regions of Helmond and The Hague expressed their willingness to participate as well (see Figure 1). The study protocol is approved by the Medical Ethics Committees of all the involved medical centres and hospitals. Patients will be randomised into an experimental group with availability of care by the nurse specialist 
or a control group receiving the usual care from their GP. Included patients will be followed during 12 months.

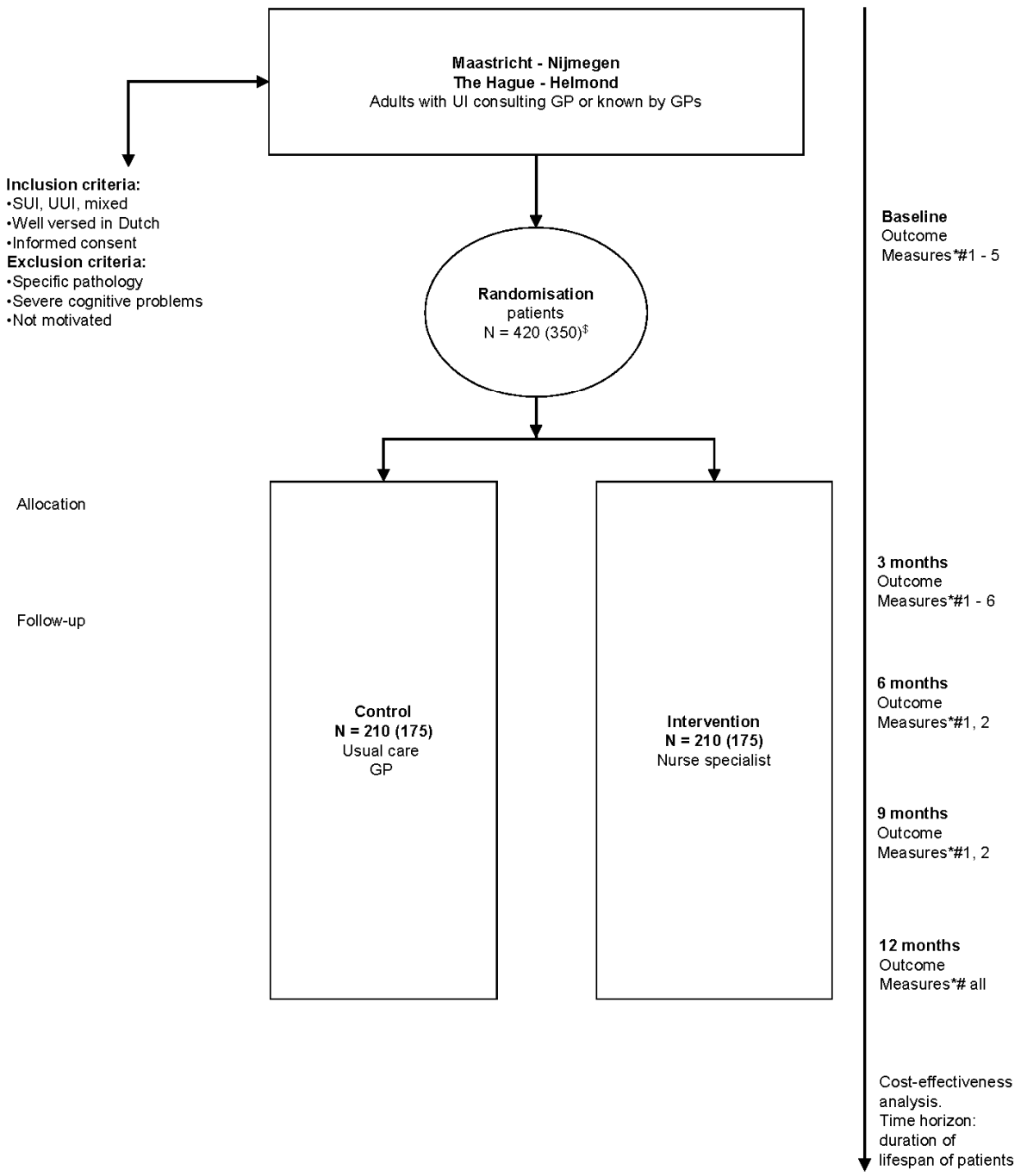

Figure 1 Study design.

* Primary outcome measure: severity urinary incontinence (ICIQ-UI SF).

\# Secundary outcome measures: 1 . healthcare and productivity, time, travel costs 2 . generic healthrelated QOL: EuroQol (EQ-5D) 3. incontinence specific QOL: IIQ-7 4. quantification of symptoms: bladder diary 5. satisfaction of patients 6 . perceptions general practitioners (GPs) and nurse specialists. $\$$ Needed number with and without drop-out/loss-to-follow up.

The randomisation process is concealed from those responsible for recruiting patients using central telephone randomisation. Because only a small number of pa- 
tients is to be expected per GP, patients are randomised using a separate randomisation list for each participating region.

Blinding patients and healthcare providers is not possible. Personnel collecting outcome data are blinded to the patients treatment allocation. A patient in the control group cannot be referred by the GP to use the route via the nurse specialist, to avoid contamination.

\section{Study population}

\section{Inclusion criteria}

All consecutive patients consulting their GP for symptoms and signs of stress, urgency and mixed UI (according to the guidelines of the Dutch College of General Practitioners on $\mathrm{UI}^{9}$ ) for a period of one year and $\mathrm{UI}$ patients diagnosed as such by the GPs in the past are eligible for the study.,24,25 Patients who consult their GP for $\mathrm{UI}$ are actively recruited by the GP to participate in the study. Known UI patients are selected by the GP on eligibility and will be invited by the GP to participate in the study. In case of doubt by the GP on eligibility the patient will be invited for a consultation on UI by the GP. Regularly and repeatedly, GPs will be stimulated to include patients for the study. Information, reminders and newsletters are part of the strategy to enhance the GPs awareness of the availability of the nurse specialist and its potential benefits for this specific healthcare.

\section{Exclusion criteria}

Excluded are patients below 18, women with prolapse degree III or more, patients with signs of reflex- or overflow incontinence, tumours in the abdomen, severe neurological diseases associated with incontinence (multiple sclerosis, CVA, diabetes, cauda equina syndrome), actual urinary tract infection, haematuria without urinary tract infection, men below 65 with unexplained incontinence, patients with failure after operation or failure of conservative therapy during the past half year (or longer, provided there is no relapse which causes dissatisfaction with the present situation), severe cognitive problems, not well versed in Dutch, refusing to participate/cooperate, patients for whom the GP considers the management via the nurse specialist as impossible/undesired and UI patients in care and nursing homes.

\section{Informed consent}

After written informed consent all patients included have baseline data collection, urologic, obstetric and gynaecologic history in women and urologic history in men. All measurements in this study are performed by postal questionnaires managed by an independent research assistant. 


\section{Intervention(s)}

The intervention is designed as close as possible to treatment options in clinical practice, including 'cascades' of patient management choices (Figure 2). When the patient is allocated to the intervention group the GP refers the patient to the nurse specialist according to a care protocol. All participating GPs are personally informed about the demarcation and definition of job responsibilities and competence profile of the nurse specialist towards GPs and other healthcare professionals involved. ${ }^{26,27}$ This information is also available to the GP as written information.

\section{Training and competencies of the nurse specialist [Additional file 1]}

The training programme for the nurse specialists how to support the GP, by taking care of the diagnostic and therapeutic management of patients with the most prevalent types of $\mathrm{UI}$, is gradually developed during the preparation period of the study. All nurse specialists will have to prove their competencies in an individual assessment.

\section{Intervention nurse specialist [Additional files 2 and 3]}

The main goal of the intervention of the nurse specialist is to provide a tailored, patient specific diagnostic and treatment plan, thereby preventing or reducing the use of incontinence pads. Based on guidelines and protocols the nurse specialist takes over tasks from the GP related to diagnostics, intervention and monitoring of patients with the most prevalent types of UI (stress, urgency and mixed UI). ${ }^{28-31}$ The GP keeps final responsibility. Furthermore, the nurse specialist supports patient motivation, compliance and adherence both on the short and the long term by monitoring patients over time in a systematic way. This ensures that patients will accept, understand, are willing and able to adhere to advices on lifestyle, bladderand pelvic floor muscle training according to a health education model. ${ }^{22}$ Another task of the nurse specialist is to give adequate information and advice about (when still necessary) the choice and the use of non-curative means like incontinence pads. She/he will always report to the GP and acts as the contact person between the other healthcare providers. In case of unclear pathology, a complex health problem or failure of treatment the nurse specialist can advise a referral to a specialist or specialised physiotherapist. In all cases, the decision for referral is to be made by the GP. Altogether this means that the nurse specialist will report to the GP on each patient after first problem assessment and at the end of the intervention period. A regular meeting between nurse specialist and GP to discuss patients will be organised when needed. 

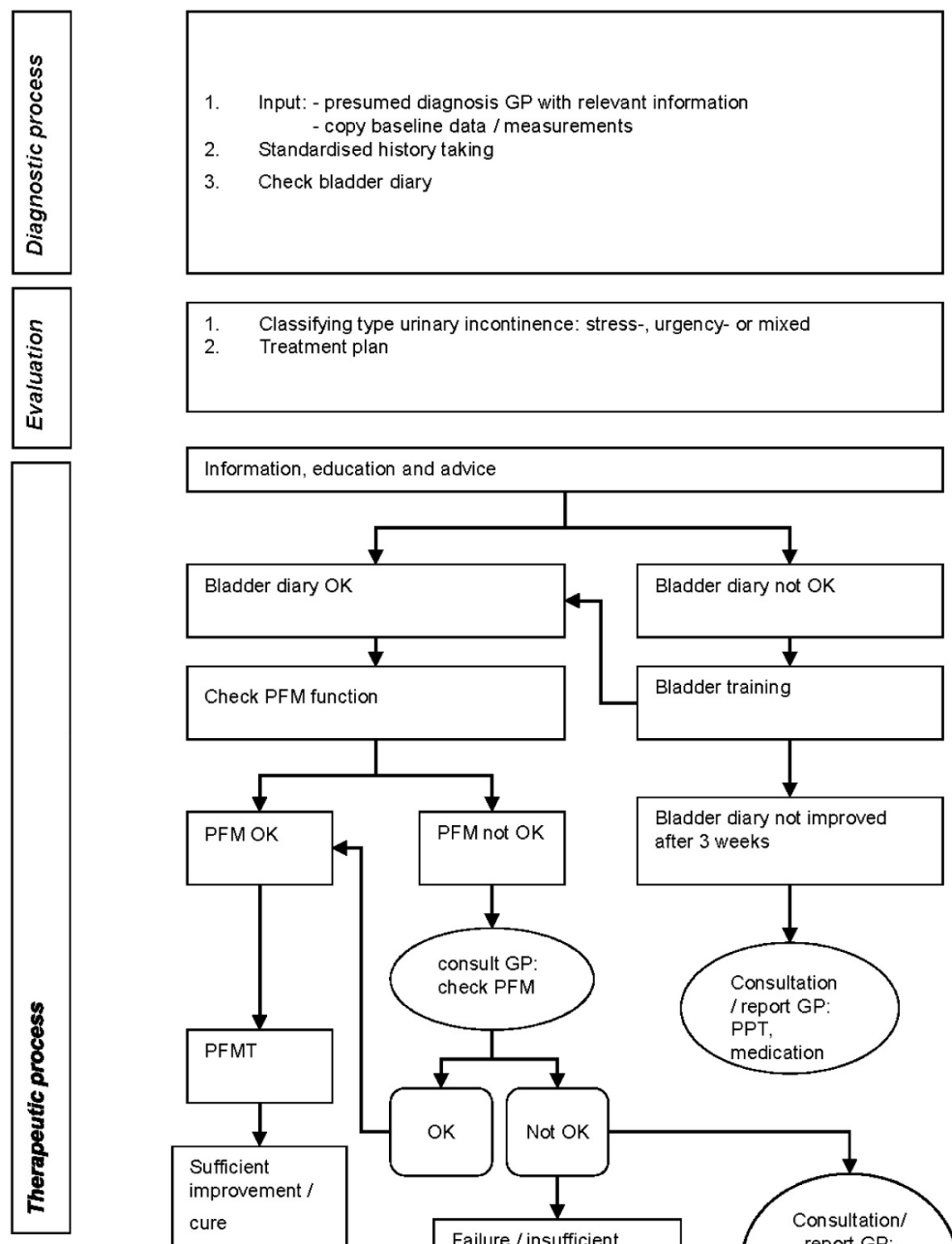

Visit 1

60 minutes

1. Classifying type urinary incontinence: stress-, urgency- or mixed 2. Treatment plan

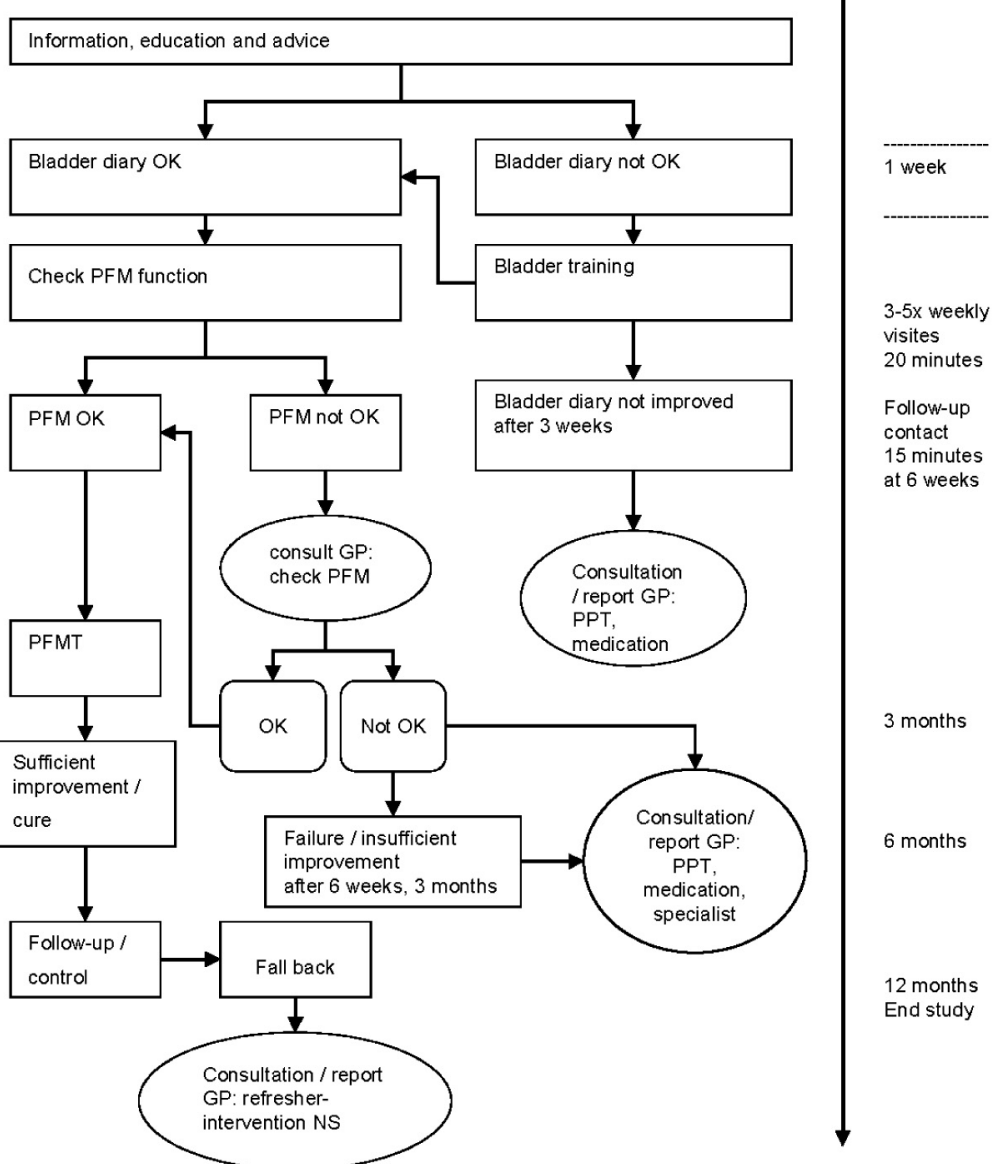

Figure 2 Flow chart intervention nurse specialist for patients with urinary incontinence.

PFM(T), Pelvic Floor Muscle (Training); GP, general practitioner; PPT, pelvic physiotherapist; NS, nurse specialist. The used terminology is according to the definitions as recommended by the International Continence Society. 


\section{Outcome measurements and data collection}

The primary outcome measure is the severity of involuntary loss of urine. This is measured by the self-completed condition-specific International Consultation on Incontinence Questionnaire Short Form (ICIQ-UI SF) which measures frequency, volume and impact on daily life of involuntary urine loss. ${ }^{32,33}$ The outcome is a sum score of the first two weighted items and the Visual Analogue Scale (VAS) score of impact on daily life. The questionnaire underwent extensive psychometric testing and is rated by the International Consultation on Incontinence (ICI) as Grade A, meaning highly recommendable. ${ }^{3}$

\section{Secondary outcome measures}

Generic quality of life is measured with the self-completed standardised EuroQol health-related quality of life questionnaire (EQ-5D). ${ }^{34}$ It provides a simple descriptive profile, a single index value for health status and a VAS scale (0-100). Health states defined by the five-dimensional descriptive system can be converted into a weighted health state index by applying scores from EQ-5D "value sets" elicited from general population samples.

Healthcare costs (the use of diagnostics, treatment and incontinence pads) and non-medical costs (productivity costs, time costs and travel costs) are collected using both registration systems and retrospective cost questionnaires. Because of recent literature and own experiences in similar trials the use of these questionnaires was favoured above cost diaries. ${ }^{35-37}$

Condition-specific quality of life is measured with the self-completed Incontinence Impact Questionnaire (IIQ). For practical reasons we used the short form (seven items) of this validated questionnaire that measures impact of urinary loss on five domains: 'mobility', 'physical functioning', 'social functioning', 'emotional health' and 'embarrassment'. ${ }^{38,39}$

Symptoms relevant for UI (the degree of pad usage, times of micturition, incontinence episodes, the degree of urgency, complications, complaints) is measured

with a self-completed bladder diary during three consecutive days. ${ }^{40-43}$ Considering the restricted diagnostic value of lower urinary tract symptoms (LUTS) for a final diagnosis (grade D) and to minimise complexity and to maximise compliance (Gordon 2001$)^{3}$ to registration of the bladder diary this registration will be restricted to a micturition time chart with the most relevant LUTS which give an impression of type, severity and impact of UI. ${ }^{3}$

Patients' satisfaction with provided care for UI by the GP and/or the nurse specialist was originally planned to be measured with the for UI adjusted QUOTE selfcompleted questionnaire. This showed to be not suitable to answer our research question. For this reason we developed a new questionnaire. For the development of the questionnaire topics relevant to either the route via the nurse specialist or 
the usual care of the GP were identified in interviews with UI patients and experts in the field participating in the study. We developed a set of items to enable us to measure and test multi-items scales. The items are related to relevant themes with respect to care for UI patients according to standards on UI.

Evaluation of the availability and involvement of the nurse specialist by GPs will be measured by interviews and questionnaires after the study in a sample of the participating GPs. Data will be collected by a trained research assistant.

Evaluation by all participating nurse specialists on role participation will be measured by a semi-structured interview and questionnaires before, during and after the study.

Data collection of all outcome measures will be done at three and 12 months. For the cost-effectiveness study extra follow-up data collection of the primary outcome measure, costs and quality of life will take place at six and nine months. In case of non-response, telephone reminders will take place two to three weeks after sending the questionnaires. Adverse events/effects will be monitored for the duration of the study.

\section{Sample size calculation and feasibility of recruitment}

Based on a mean score on the ICIQ-UI SF ${ }^{44}$ of 7.18 (SD 6.64), and an expected improvement of 2 on the outcome scale from 0 to 21 (which gives a delta value of $2 / 6.64=0.301$ ), a power of $80 \%$ and a significance level of 0.05 , and given the twosided H1-hypothesis, that the new professional improves the effect, the needed number of patients per arm is 175 patients. We expect a drop-out rate during the trial of $20 \%$ and therefore we set our target at (rounded off) two times $210=420$ patients. As shown in previous Dutch studies on UI, GP practices are able to include five to 10 patients per year. Therefore, we need 50 to 88 GPs to participate. Recruitment of the needed numbers of patients seems highly feasible since the power calculation was based on the regions of Maastricht and Nijmegen alone (total number of inhabitants: 500,000; total number of GP practices: approximately 240 GPs).

\section{Data analysis and presentation / synthesis}

Data collection, processing and analyses will be done with SPSS/PC, version 12 and 15. Variables will be analysed with the parametric Student's t-test or the nonparametric U-test of Mann-Whitney (Wilcoxon ranksumtest) and the Wilcoxon-test for two unpaired samples. To compare the effects between different groups repeated measures AN(C)OVA and linear regression models will be used. Analyses will be done according to the intention-to-treat-principle. In case of missing values, noncompliance, loss to-follow-up, drop-outs and protocol deviations also per-protocol 
analyses will be done for the economic evaluation only. To compare GPs using the nurse specialist and GPs who do not, per-protocol analyses will be performed.

\section{Economic evaluation}

A cost-effectiveness analysis will be performed from the societal perspective. In this analysis the incremental health effects and the incremental costs of involving a nurse specialist in primary care for adult patients with UI, as compared to usual care, will be weighted. The major potential health effects of involving a nurse specialist are likely to result from an increase in the utilisation of available therapies with proven effectiveness for UI, such as bladder training and/or pelvic floor muscle training. The EuroQol will be used to calculate Quality Adjusted Life Years (QALYs) following the algorithm developed by Dolan et al. ${ }^{45}$ Healthcare costs, productivity costs and patient and family costs will be included in the analysis. Healthcare costs (the use of diagnostics, treatment and incontinence pads) and productivity costs, time costs and travel costs will be collected using both registration systems and retrospective cost questionnaires. The cost questionnaire will be filled in by the patients at baseline, three, six, nine and 12 months. Cost calculation will be based on real prices or on unit prices from the Dutch Guideline for Cost Calculation. ${ }^{46}$ For the nurse specialist a cost price calculation will be performed. Costs associated with productivity loss will be calculated using the friction costs method. ${ }^{46}$ In case of household, or other unpaid activities, shadow prices will be used. When clinical relevant effects of decreasing the impact of UI on daily life, and thus the use of incontinence pads, will be found during the one year of the study, a Markov type health state transition model will be used to calculate the long-term costeffectiveness of the intervention [Additional file 4].

\section{Discussion}

In this paper we describe the study protocol of, to our knowledge, the first randomised controlled trial on care for UI patients in general practice that includes a full economic evaluation and cost-effectiveness modelling exercise from the societal perspective. Next to this, despite the growing emphasis in the Netherlands on involving nurse specialists in general practice, specifically for UI this is still unfamiliar and the delegation of tasks to a nurse specialist can be considered as a new approach. $^{17,47}$ 
(Potential) strengths of the study protocol

\section{The study design}

The choice for a pragmatic design ensures that the intervention is as close as possible to treatment options in clinical practice (including 'cascades' of patient management choices). This makes implementation in the future easier. A care protocol with the preferred route via the nurse specialist is used. GPs will be well informed and stimulated to follow this care protocol. Despite this, there is a chance that GPs will not optimally use the nurse specialist because of perceptions of GPs or unfamiliarity with this new professional. For this reason perceptions such as willingness to use the nurse specialist, expectations, first experiences and reasons/promoting or hampering factors for GPs to use or not to use this route will be measured and actively monitored.

\section{Recruitment strategy}

A particular strength is the recruitment strategy. Both new and already diagnosed UI patients are eligible. By recruiting already diagnosed UI patients we are likely to also include the majority of UI patients who mainly use pads and did not receive adequate conservative treatment before. In addition, this approach will be helpful to include the required number of patients in the available time period.

\section{Randomisation approach}

A third strength of our study protocol is the randomisation approach, in which allocation is concealed and done by an external independent person. Randomisation is done on patient level. An alternative would be that GPs would be randomised instead of patients. As GPs in the control group probably will have difficulties to include enough patients, this alternative seemed unfavourable.

\section{Competencies of the nurse specialists}

Another strength of our study protocol is that all nurses are trained and assessed in a uniform way to assure that the intervention will be carried out conform the intervention protocol in all four regions. To be able to get insight into their actual performance, all nurses will register their actions during all patient visits. This registration will be used to search for factors related to the intervention process that might influence the effectiveness of the intervention. It may also show an individual learning curve, as this intervention is new for the nurses.

\section{Outcome measurements}

Diagnosing the type of UI is primarily done by the GP. Since we really want to mimic routine practice as close as possible we did not choose for objective measurements 
to confirm the diagnosis of the type of UI by urodynamic testing. Neither did we choose for a pad test to measure the severity of UI. On the one hand because we felt that a pad test would be too much of a burden especially for the control group only receiving usual care. On the other hand because a pad test may act as a feedback which might influence the usual care too much. Furthermore, in the analyses by taking the baseline scores into account the information bias resulting from subjective self-reports will be reduced. Moreover, the self-reported data on healthcare use and costs will be validated by data from external sources like insurance companies (patients will give their informed consent).

\section{(Potential) limitations of the study protocol}

\section{Participant selection}

Selection of participants may limit generalisation of the results of this study as selective non-response of patients as well as selective drop-out and selective refusal of GPs are possible. Selective refusal of patients to participate might be assumed as some patients label UI as an accepted aspect of normal ageing or do not experience adverse consequences. Possibly, such UI patients will refuse more often to participate than other UI patients. The same might be assumed about the more complex UI patients, who are severely burdened by multi-morbidity, and might be afraid to become even more burdened with participating in the study measurements. To limit such selective refusal patients living in care- and nursing-homes are excluded from the study. To prevent selective drop-out of patients in poor health states, the questionnaires are designed to minimise completion burden. Selective refusal of GPs to participate is anticipated from GPs already working with (in)continence nurses or specialised physiotherapists. Randomisation on patient level could lead to contamination, and bias the results of this study. However, the influence of contamination is minimised, because patients in the care-as-usual group have no access to the intervention of the nurse specialist. Nevertheless, it is possible that participating GPs are encouraged by the study to give more attention to the UI patients participating in the usual care group. As a result our findings may be conservative.

\section{Conclusion}

The study will provide evidence whether the availability of a nurse specialist in a new role as substitute for the GP leads to more effective and efficient care for adult patients with UI. Furthermore, the results will show whether this availability of nurse specialists improves the quality of life of patients and the satisfaction of patients, GPs and other care providers. If this intervention proves to be effective, im- 
plementation of this intervention is considered and anticipated. First results of this study will become available autumn 2008.

\section{Acknowledgements}

The Netherlands Organisation for Health Research and Development (ZonMw) funded this study. Health Care Efficiency Research program, Sub-program 2: Effects and Costs, grant number 945-04-224. We would like to thank Zorg Groep ZuidGelderland, The Netherlands, Groene Kruis Domicura, Maastricht, The Netherlands, AZIVO/ PCC of Leyenburg Hospital, The Hague, The Netherlands, De Zorgboog, Quartz of the Elkerliek Hospital, Helmond, The Netherlands, for their participation in the set-up of the study and the availability of their nurses; we would like to thank all participating GPs (and related pharmacists, MediReva V.O.F., Medithuis and CZ) for their cooperation in this project. 


\section{References}

1. GIP/Health Insurance Board. Total cost 2002-2006. 2007.

2. Teunissen TAM, Bosch van den WJHM, Hoogen van den HJM, Lagro-Janssen ALM. Prevalence of urinary, fecal and double incontinence in the elderly living at home. Int Urogynecol J 2004, 15(1):10-13.

3. Abrams P, Cardozo L, Khoury S, Wein A. Incontinence. 3rd International Consultation on Incontinence. Volume 1\&2. Monaco: Health Publication Ltd. 2005.

4. Norton P, Brubaker L. Urinary incontinence in women. Lancet. 2006; 367(9504):57-67.

5. Health Council of the Netherlands (Gezondheidsraad). Urine Incontinentie [Urinary Incontinence]. Publication no. 2001/12. The Hague 2001. Report No.: ISBN:90-5549-375-9.

6. Health Insurance Board (CVZ). Urine incontinentie bij volwassenen [Urinary Incontinence in adults]. Diemen 2003 Contract No.: 2003/147.

7. Melville JL, Katon W, Delaney K, Newton K. Urinary Incontinence in US Women: A Population-Based Study. Arch Intern Med 2005, 165(5):537-542.

8. Newman DK, Denis L, Gruenwald I, Ee CH, Millard R, Roberts R, Sampselle C, Williams, K. Continence Promotion: prevention, education and organisation. In Incontinence 3nd International Consultation on Incontinence. Edited by Abrams P, et al. Plymouth: Health Publication Ltd; 2005.

9. Lagro-Janssen ALM, Teunissen D, Breedveldt-Boer HP, Dongen van JJAM, Lemain TJJ, Steeneken F, Dijkstra RH, Wiersma TJ. NHG-Standaard Incontinentie voor Urine M46 Eerste herziening (Dutch College Guideline on Urinary Incontinence. First revision). Huisarts Wet 2006, 49(2):501-510.

10. Kirby M. Managing stress urinary incontinence - a primary care issue. Int J Clin Pract 2006, 60(2):184189.

11. Albers-Heitner P, Berghmans B, Nieman F, Lagro-Janssen T, Winkens R. Adherence to professional guidelines for patients with urinary incontinence by general practitioners: a cross-sectional study. J Eval Clin Pract.2008;14(5):80-11.

12. Albers-Heitner $P$, Berghmans B, Nieman F, Lagro-Janssen $T$, Winkens R. How do patients with urinary incontinence perceive care given by their general practitioner? A cross-sectional study. Int J Clin Pract. 2008; 62(3):508-515.

13. Westert GP, Schellevis FG, de Bakker DH, Groenewegen PP, Bensing JM, Zee vd J. Monitoring health inequalities through general practice: the Second Dutch National Survey of General Practice. Eur J Public Health. 2005; 15(1):59-65.

14. Faber E, Custers JWH, Berghmans LCM, Dongen van JJAM, Groeningen van COM, Grupping-Morel MHM, Hogen Esch FAH, Lagro-Janssen ALM, Pinxteren van B, Verstappen WHIM. Landelijke Eerstelijns Samenwerkings Afspraak Incontinentie voor Urine (National First Line Multidisciplinary Agreement) 'LESA'. Huisarts Wet. 2007; 50(5):S9-S12.

15. Grol R, Buchan H. Clinical guidelines: what can we do to increase their use? Med J Aust. 2006; 185 (6):301-302.

16. Cabana MD, Rand CS, Powe NR, Wu AW, Wilson MH, Abboud P-AC, Rubin HR. Why Don't Physicians Follow Clinical Practice Guidelines?: A Framework for Improvement. JAMA. 1999; 282(15):1458-1465.

17. Vrijhoef HJM. Is it justifiable to treat chronic patients by nurse specialists? PhD Thesis. Maastricht University, Department of General Practice; 2002.

18. Berg van den M, Bakker de D. Meta-analyse introductie praktijkondersteuning op HBO-niveau in de huisartspraktijk in Nederland. NIVEL; 2003.

19. Borrie M, Bawden M, Speechley M, Kloseck M. Interventions led by nurse continence advisers in the management of urinary incontinence: a randomized controlled trial. CMAJ. 2002;166((10):1267-1273.

20. O'Brien J, Long $\mathrm{H}$ : Urinary incontinence: long term effectiveness of nursing intervention in primary care. BMJ. 1995; 311(7014):1208.

21. Berghmans LCM, Hendriks HJM, Bø K, Hay-Smith EJ, Bie de RA, Waalwijk van Doorn van ESC. Conservative treatment of stress urinary incontinence in women: a systematic review of randomized clinical trials. Br J Urol. 1998; 82:181-191. 
22. Williams KS, Assassa RP, Smith NK, Jagger C, Perry S, Shaw C, Dallosso H, McGrother C, Clarke M, Brittain KR, Castleden CM, Mayne C. Development, implementation and evaluation of a new nurseled continence service: a pilot-study. J Clin Nursing. 2000; 9:566-573.

23. Williams KS, Assassa RP, Cooper NJ, Turner DA, Shaw C, Abrams KR, Mayne C, Jagger C, Matthews R, Clarke $\mathrm{M}$ et al. Clinical and cost-effectiveness of a new nurse-led continence service: a randomised controlled trial. Br J Gen Pract. 2005; 55(518):696-703

24. Abrams P, Cardozo L, Fall M, Griffits D, Rosier P, Ulmsten U, Kerrebroeck van P, Victor A, Wein A. The standardisation of Terminology of Lower Urinary Tract function: Report from the Standardisation Sub-committee of the International Continence Society. Neurourol Urodynam. 2002; 21:167-178.

25. Brown JS, Bradley CS, Subak LL, Richter HE, Kraus SR, Brubaker L. Diagnostic aspects of Incontinence Study (DAISy) Research Group. The sensitivity and specificity of a simple test to distinguish between urge and stress urinary incontinence. Ann Intern Med. 2006; 144:715-723.

26. NVFB. Functieprofiel Bekkenfysiotherapeut. Dutch Association for Physical Therapy for Pelvic Floor Disorders and Pre- and Postnatal Healthcare (NVFB); 2003:56.

27. VNIV. Incontinentieverpleegkundige. Beroepsdeelprofiel. Utrecht: Vereniging Nederlandse Incontinentie Verpleegkundigen (VNIV); 2004:28.

28. Verhulst FJCM, Burgt van der MCA, Lindner K. Concretisering van patiëntenvoorlichting in het fysiotherapeutisch handelen. Ned T Fysio. 1994; 1:10-17.

29. Damoiseaux V, Molen van der HT, Kok GJ. Gezondheidsvoorlichting en gedragsverandering. Assen: Van Gorcum \& Comp B.V.; 1993.

30. Messelink EJ, Bemelmans BLH, Groot JAM, Gisolf KWH, Vervest HAM, Berghmans LCM, Everaerd WTAM, Kil PJM, Wiersma T. Urge en urge-incontinentie. Utrecht: Nederlandse Vereniging voor Urologie (NVU); 2002.

31. Venema PL, Geelen van JM, Kil PJM, Capelle van JW, Tjon Pian Gi NP, Ridder de D, Wiersma T. Richtlijn Stressincontinentie bij de vrouw (Guideline Stress Incontinence in women). Utrecht: Nederlandse Vereniging voor Urologie (NVU); 2003.

32. Donovan J, Badia X, Corcos J, Gotoh M, Kelleher C, Naughton MJ, Shaw C. Symptom and quality of life assessment, July 1-3, 2001, 2nd edn. In Incontinence: Proceedings of the Second International Consultation on Incontinence. Edited by Cardozo L, Khoury, S. \& Wein, A. . Plymouth: Health Publication Ltd; 2002:267-316.

33. Avery K, Donovan J, Peters T, Shaw C, Gotoh M, Abrams P. ICl-Q: a brief and robust measure for evaluating the symptoms and impact of urinary incontinence. Neurourol Urodynam. 2004; 23(4):322-330.

34. Euroqol Group. Euroqol - a new facility for the measurement of health related quality of life. Health Policy. 1990; 16:199-208.

35. Hessel FP, Wittmann M, Petro W, Wasem J. Methoden zur kostenverfassung im Rahmen der okonomischen evaluation einer rehabilitationsmassnahme bei chronischen atemwegserkrankungen. Pneumologie. 2000; 54:289-295.

36. Koopmanschap MA, Meerding WJ, Evers S, Severens J, Burdorf A, Brouwer W. Handleiding voor het gebruik van PRODISQ versie 2.1 (PROductivity and DISease Questionnaire). Een modulaire vragenlijst over de relatie tussen ziekte en productiviteitskosten. Toepasbaar bij economische evaluaties van gezondheidszorgprogramma's voor patiënten en werknemers. Rotterdam/Maastricht; 2004.

37. Brink van den M, Hout van den WB, Stiggelbout AM, Velde van de CJH, Kievit J. Cost measurement in economic evaluations of health care. Whom to ask? Med Care. 2004; 42(8):740-746.

38. Uebersax JS, Wyman JF, Shumaker SA, McClish DK, Fantl JA, and the Continence Program for Women Research Group. Short forms to assess life quality and symptom distress for urinary incontinence in women: the Incontinence Impact Questionnaire and the urogenital Distress Inventory. Neurourol Urodynam. 1995; 14:131-139.

39. Vaart van der $\mathrm{CH}$, Leeuw de JRJ, Roovers JPWR, Heintz APM. De invloed van urine-incontinentie op de kwaliteit van leven bij thuiswonende Nederlandse vrouwen van 45-70 jaar. Ned Tijdschr Geneeskd. 2000; 144(19):894-897. 
40. Blaivas JG, Appell RA, Fantl JA, Leach G, McGuire EJ, Resnick NM, Raz S, Wein AJ. Standards of efficacy for evaluation of treatment outcomes in urinary incontinence: recommendations of the Urodynamic Society. Neurourol Urodynam. 1997; 16(3):145-147.

41. Blaivas JG, Appell RA, Fantl JA, Leach G, McGuire EJ, Resnick NM, Raz S, Wein AJ. Definition and classification of urinary incontinence: recommendations of the Urodynamic Society. Neurourol Urodynam. 1997; 16(3):149-151.

42. Mattiasson A, Djurhus JC, Fonda D, Lose G, Nordling J, Stöhrer M. Standardization of outcome studies in patiënts with lower urinary tract dysfunction: a report on general principles from the standardization committee of the International Continence Society. Neurourol Urodynam. 1998; 17:249-253.

43. Blaivas J. Outcome measures for urinary incontinence. Urology. 1998; 51(supplement 2A):11.

44. Abrams P, Cardozo L, Khoury S, Wein A. Incontinence Proceedings 2nd International Consultation on Incontinence - July 1, 2001- July 3, Paris: Health Publication Ltd; 2002.

45. Dolan P. Modeling Valuations for EuroQol Health States. Med Care. 1997; 35(11):1095-1108.

46. Oostenbrink JB, Bouwmans CM, Koopmanschap MA, Rutten FFH. Manual for Cost Analysis. Amstelveen: College voor Zorgverzekeringen. (In Dutch); 2004.

47. Laurant M, Reeves D, Hermens R, Braspenning J, Grol R, Sibbald B. Substitution of doctors by nurses in primary care. Cochrane Database of Systematic Reviews; 2004, Issue 4. DOI: 10.1002/14651858.CD001271.pub2. 


\section{Additional file 1 Training and competencies of the nurse specialist}

The intervention means incorporating a nurse specialist to support the GP, by taking care of the diagnostic and therapeutic management (based on guidelines) of patients with the most prevalent types of UI (stress UI, urgency UI and mixed UI).

During two days of seven hour sessions with at least one week interval, each participating nurse will be trained by specialists in the field of UI (a general practitioner, a specialised pelvic physiotherapist and a continence nurse), how to take over tasks related to diagnostics, intervention and monitoring of incontinence from the GP based on guidelines and protocols. As mentioned earlier, UI is especially a problem for elderly women. In this population group the problem is often more complex and concurrent co-morbidities related to $\mathrm{UI}$ result in discomfort and handicap. $^{2}$ So, almost automatically, the higher prevalence, nature and severity of the health problem will affect the work of the nurse specialist. During the training of the nurse specialist, special attention will be given to the special needs of this population group. The same applies for the potential impact of cultural background.

In between and after the training days, the nurses have to practice their newly learned skills on pilot patients, will visit an experienced continence nurse, a specialised pelvic physiotherapist and a urology department to get insight in demarcation of the different specialties dealing with UI patients. Next to this they will have to spend time to prepare and organise the necessary materials and accommodation for the intervention. Finally, in a two and half hour session each individual nurse must prove her competencies both in an individual written assessment and an assessment with a simulation patient. Because this is a new intervention, before and during the time of the study all participating nurse specialists will meet on a regular base for intervision both with each other and specialists in this field of the research team. These specialists can be contacted to discuss cases at any time. Since this nurse specialist is a new profession a learning curve is to be expected.

\footnotetext{
${ }^{2}$ Lagro-Janssen ALM, Teunissen D, Breedveldt-Boer HP, Dongen van JJAM, Lemain TJJ, Steeneken F, Dijkstra RH, Wiersma TJ. NHG-Standaard Incontinentie voor Urine M46 Eerste herziening (Dutch College Guideline on Urinary Incontinence. First revision). Huisarts Wet. 2006, 49(2):501-510.
} 


\section{Additional file 2 Intervention nurse specialist: diagnostic process, evaluation and therapeutic process}

\section{First visit}

\section{Diagnostic process}

Input: presumed diagnosis GP with relevant information; copy of baseline data and measurements.

Standardised history taking: registration of problems in pelvic floor/bladder function (impairment), activities (disability) and participation problems and the influence of personal and external factors. (International Classification of Functioning, Disability and Health-ICF). ${ }^{3}$ Check bladder diary.

\section{Evaluation}

Classifying type of incontinence: stress-, urgency- or mixed urinary incontinence.

Treatment plan: the nurse specialist discusses and provides relevant information to the patient about the treatment plan, with respect to the diagnosed problem and the cognitive and physical possibilities of the patient. She points out her task and what is expected from the patient. The nurse specialist can use bladder diaries and 24 hours home pad tests during daily activity as evaluation and feedback instruments.

\section{Therapeutic process}

The nurse specialist follows the next more or less standardised treatment plan based on best available evidence:

- Information, education and advice on anatomy and function of bladder and pelvic floor muscles, good toilet behaviour, influence lifestyle and, when appropriate, mobility, co-morbidity and cognition on urinary incontinence.

- When bladder diary abnormal: continue first with advice on bladder training; repeat diary. When bladder diary not improved after 3 weeks: consult GP. When bladder diary normal: continue with advice on pelvic floor muscle (PFM) function.

- Assess the functional possibilities of the PFM of the patient: the patient checks at home awareness of PFM function:

- contraction: ability to postpone micturition for maximally 10 seconds;

- relaxation: ability to initiate and continue micturition with a good flow without hesitation and/or interruption.

\footnotetext{
${ }^{3}$ World Health Assembly Resolution 54.21.(2001) International Classification of Functioning, Disability and Health (ICF). http://www.who.int/classifications/icf/en/(accessed may 2004).
} 


\section{Follow-up visit(s)}

- Patient is aware and in control over this function of the PFM: continue advice on adequate use of PFM function and training. Patient is not aware or in control of the PFM: the nurse specialist consults the GP to check PFM function by digital vaginal and/or rectal palpation.

Awareness and control improved: the nurse specialist continues PFM training (PFMT).

Still no or insufficient awareness and/or control after check GP or after 6 weeks: the nurse specialist consults the GP.

- Advice on incontinence pads or other non-curative materials adjusted to individual needs and wishes of the patient and to prevent problems as a result of the used materials. The main goal of the nurse specialist is to check whether the use of the means and pads is needed or can be reduced by information, advice and bladder training and/or PFMT.

- Effect on urinary incontinence after three months:

- insufficient improvement or failure (no cure): consult GP;

- sufficient improvement or cure: follow-up patients to stimulate compliance and prevent fall back and thereby improving the long-term effect of the intervention during the first year after start of the intervention. Patients can either contact the nurse specialist when they experience fall back or are actively contacted by the nurse specialist at six and 12 months after start of the intervention.

In case of fall back the nurse specialist considers together with the patient a consult with the GP for a refresher intervention. 


\section{Additional file 3 Description intervention nurse specialist}

Information, education and advice about

- Anatomy and function of the pelvic floor and pelvic organs in general and specific with activities of daily life (weight bearing possibilities of the pelvic floor)

- Healthy toilet behaviour (relate to findings bladder diary);

- Lifestyle influence on incontinence: weight control, smoking, dietary factors (fluid intake: relate to bladder diary), constipation, postural changes, hygiene, etc. (the load);

- The role of co-morbidity, dexterity and mobility, cognition and ageing, hormonal status.

\section{Check bladder diary on normal toilet behaviour}

Micturition daytime 5-7 times, night time 0-2 times with normal fluid intake 11/2- 2 litres daily. Normal maximally voided volume for adults varies from 300 to $700 \mathrm{ml}$, control mean voided volumes. Relaxed micturition without straining.

\section{Bladder training}

Mainly based on the signs and symptoms and the diagnostic procedures, such as the bladder diary. The aim of bladder training is normalising frequency of micturition. When patients go infrequently or irregularly stimulate to go more frequent with regular two to three hour voiding intervals. When frequency is over seven times daily then, on the basis of extrapolation from the bladder retraining literature, the outpatient retraining protocol includes an initial voiding interval typically beginning at one hour during waking hours, which is increased by 15 to 30 minutes per week depending on tolerance of the schedule (i.e., fewer incontinent episodes than the previous week, minimal interruptions to the schedule, and the patients feeling of control over urgency), until a two to three hour voiding interval is achieved. A shorter initial voiding interval, i.e., 30 minutes or less, may be necessary for patients whose baseline urinary diaries reveal an average voiding interval of less than one hour. Education will be provided about normal bladder control and methods to control urgency such as distraction and relaxation techniques and pelvic floor muscle contraction. Self-monitoring of voiding behaviour using bladder diary and treatment log will be included in order to determine adherence to the schedule, evaluate progress, and determine whether the voiding interval should be changed. The nurse specialist monitors progress, determines adjustments to the voiding interval, and provides positive reinforcement to patients undergoing bladder retraining weekly (minimum three weeks 20 minutes visits) during the training period (follow-up 15 minute visits at six weeks, three, six and 12 months). 
Control and advice on Pelvic Floor Muscle function (PFMF) and Pelvic Floor Muscle Training (PFMT):

Since legal restraints do not allow the nurse specialist to examine the actual PFM she/he has to give an exercise advice based on the patients observations of contraction and relaxation as formulated in the first visit. If the patient is able to do so, explanation about the functional use and training of the PFM as follows:

Stress urinary incontinence: PFM must be contracted to improve closure of the urethra whenever the pelvic floor is loaded either by coughing, lifting, jumping and with activities where the patient experiences urinary incontinence. Contractions may be quick and short or prolonged with power depending on the activity.

Urgency urinary incontinence: the PFM is used to inhibit detrusor overactivity, this can be short and quick or prolonged.

In case of mixed urinary incontinence PFMT is used for both goals with inhibition of detrusor overactivity as the first goal. The nurse specialist monitors progress, determines adjustments to the PFMT, and provides positive reinforcement to patients undergoing PFMT weekly (minimum three weeks 20 minutes visits) during the training period (follow-up 15 minute visits at six weeks, three, six and 12 months).

Overview treatment plan

\begin{tabular}{lll}
\hline Stress urinary incontinence & Urgency urinary incontinence & Mixed urinary incontinence \\
\hline Toilet behaviour & Bladder training & Bladder training \\
PFMT: improve closure mecha- & PFMT: inhibition bladder activity & PFMT: inhibition bladder activity \\
nism & If necessary: advice & PFMT: improve closure mecha- \\
If necessary: advice & means/materials & nism \\
means/materials & & If necessary: advice \\
& & means/materials \\
\hline
\end{tabular}




\section{Additional file 4 Modelling approach}

When clinical relevant effects of decreasing the impact of UI on daily life, and thus the use of incontinence pads, will be found during the one year of the study, a Markov type health state transition model will be used to calculate the long-term costeffectiveness of the intervention.

The population in the model consists of patients who consult(ed) their GP with complaints of UI. Relevant health states for the model will be defined, and patient time spent in these different health states will be calculated. Time spent in the health states will be weighted against both generic quality of life (EuroQol) and experiencing incontinence specific problems (ICIQ-UI SF). Cost-effectiveness acceptability curves will be determined to indicate the probability of cost-effectiveness given varying levels of the society's willingness to pay for a QALY. All model parameters will be based on outcomes observed in the underlying study or an extensive literature review. When no data is available, or when the data is insufficiently reliable, parameter estimates will be derived from panel sessions with leading experts in the field. Cycle length will be one month. One way sensitivity analysis will be performed to establish the separate effect of model parameters on the results of the analysis. The model parameters will be varied across a plausible range. Parameter uncertainty will be further tested using probabilistic sensitivity analysis. Probabilistic sensitivity analysis takes into account the fact that some combinations of factors are more likely to take place than others. The population in the model will be followed until the last patient has deceased. Excel spreadsheet modelling will be used to perform the calculations. Future costs and effects will be discounted according to Dutch guidelines. ${ }^{4}$

\footnotetext{
${ }^{4}$ Oostenbrink JB, Bouwmans CM, Koopmanschap MA, Rutten FFH. Handleiding voor kostenonderzoek [Manual for Cost Analysis]. Amstelveen: College voor Zorgverzekeringen. (In Dutch), 2004.
} 



\title{
Chapter 5
}

\section{Effectiveness of involving a nurse specialist for patients with urinary incontinence in primary care:}

Results of a pragmatic multicentre randomised controlled trial

\author{
Pytha Albers-Heitner \\ Toine Lagro-Janssen \\ Manuela Joore \\ Bary Berghmans \\ Fred Nieman \\ Pieter Venema \\ Johan Severens \\ Ron Winkens
}

The International Journal of Clinical Practice. 2011;65(6):705-712.

Linked Comment: www.youtube.com/IJCPeditorial.

Linked Comment: Michie. Int J Clin Pract 2011;65:635-7. 


\section{Abstract}

Background Urinary incontinence (UI) primary care management is substandard, offering care rather than cure despite the existence of guidelines that help to improve cure. Involving nurse specialists on incontinence in general practice could be a way to improve care for UI patients.

Aims We studied whether involving nurse specialists on $\mathrm{UI}$ in general practice reduced severity and impact of $U$ I.

Methods Between 2005 and 2008 a pragmatic multicentre randomised controlled trial was performed comparing a one-year intervention by trained nurse specialists with care-as-usual after initial diagnosis and assessment by general practitioners in adult patients with stress, urgency or mixed $\mathrm{UI}$ in four Dutch regions (Maastricht, Nijmegen, Helmond, The Hague). Simple randomisation was computergenerated with allocation concealment. Analysis was performed by intention-totreat principles. Main outcome measure was the International Consultation on Incontinence Questionnaire Short Form (ICIQ-UI SF) severity sum score.

Results A total of 186 patients followed the intervention and 198 received care-as-usual. Patients in both study groups improved significantly in UI severity and impact on health-related quality of life. After correction for effect modifiers (type of $\mathrm{UI}$, body mass index [BMI]) we found significant differences between groups in favour of the intervention group at three months $(p=0.04)$; no differences were found in the one-year linear trend $(p=0.15)$. Patients in the intervention group without baseline anxiety/depression improved significantly better compared with care-as-usual after one year $(p=0.03)$.

Conclusion Involving nurse specialists in care for UI patients supplementary to general practitioners can improve severity and impact of $\mathrm{UI}$, after correction for effect modifiers. This is also the case in specific situations such as anxiety/depression. 


\section{Introduction}

Urinary incontinence (UI) is a frequent clinical condition. The estimated prevalence of 'any UI' in middle-aged and older women in the general population appears to be in the range of $30 \%$ to $50 \%$ (increasing with age). Ul is at least twice as prevalent in women as compared with men. ${ }^{1}$ Considering the ageing population a further increase is expected. ${ }^{1} \mathrm{UI}$ is infamous for its impact on general well-being and social activities. If not treated and monitored, $\mathrm{UI}$ is a chronic dynamic disorder, complicated by co-morbidities, with a strong tendency to increase over time. ${ }^{1,2}$ National and international primary care guidelines on UI indicate that for most UI patients pelvic floor muscle and/or bladder training is the best non-invasive initial treatment to solve the problem. ${ }^{2,3}$ So far, despite guidelines, training is only incidentally offered. ${ }^{4-6}$ Most GPs choose a non-curative alternative: prescribing incontinence pads. More than $50 \%$ of UI patients use incontinence pads, especially older people.,

Although UI threatens health-related quality of life, it is not life threatening. ${ }^{6,7}$ Adequate treatment of UI can be complex and time consuming. So far, UI has not been receiving much attention in general practice, despite the large number of patients who suffer from it. Probably, there are several reasons, such as lack of knowledge, other clinical priorities, lack of time and lack of targeted implementation strategies to adopt and practice guideline recommendations. ${ }^{4,5,8-12}$ Therefore, employing nurse specialists may offer a solution to improve the quality of care in general practice provided to UI patients. We envisioned that nurse specialists, after thorough training, would be well equipped to support GPs after the initial consultation and assessment of a UI patient by the GP. Nurse specialists have specific skills and have extra time to inform and motivate patients compared to GPs. Their general acceptance by patients and GPs, feasibility, usefulness in management ${ }^{13,14}$ and the specific effectiveness in treating $\mathrm{UI}$ have been reported. ${ }^{15}$

However, little information is available from randomised controlled trials (RCT) with long-term follow-up on the effectiveness of involving nurse specialists for UI compared with usual care in general practice. ${ }^{15,16}$ Therefore, we set up a trial to study whether involving nurse specialists for UI could improve the quality of care for adult $\mathrm{UI}$ patients in general practice. ${ }^{17}$ We envisioned that this change in process of care would also improve quality of care in terms of treatment outcome.

We addressed the following main research question: "Does the involvement of a nurse specialist for adult persons with UI reduce the overall UI severity including symptoms of frequency, volume and the impact on the health-related quality of life compared with care-as-usual?". 


\section{Materials and Methods}

From May 2005 until March 2008, we performed a pragmatic multicentre RCT comparing UI patients (randomly assigned following simple randomisation procedures) receiving nurse specialist care with $\mathrm{UI}$ patients receiving care-as-usual in general practice in four Dutch regions (Maastricht, Nijmegen, Helmond, The Hague). A detailed study protocol and description of the intervention is reported elsewhere. ${ }^{17}$ In short, adult patients with stress, urgency or mixed UI, already known or newly diagnosed by their GP, were eligible and actively recruited by their GP to participate in the study and followed during one year. To identify patients known to suffer from UI, GPs searched in their electronic medical record system using the International Classification of Primary Care (ICPC) coding system and obtained a list with pad users from the local pharmacies. Patients who met the inclusion and exclusion criteria were invited to participate in the trial (Table 1). Randomisation was computergenerated, with allocation concealment by sealed envelopes. Blinding patients and healthcare providers was obviously not possible. Based on a mean UI severity sum score on our primary outcome, the International Consultation on Incontinence Questionnaire Short Form (ICIQ-UI SF), ${ }^{18}$ of 7.18 (SD 6.64), an expected clinical important improvement of two points on the outcome scale (delta value of 2/6.64 = 0.301 ), a power of $80 \%$ and a two-sided significance level of 0.05 , we needed 175 patients per arm, 350 in total.

Table 1 Inclusion and exclusion criteria.

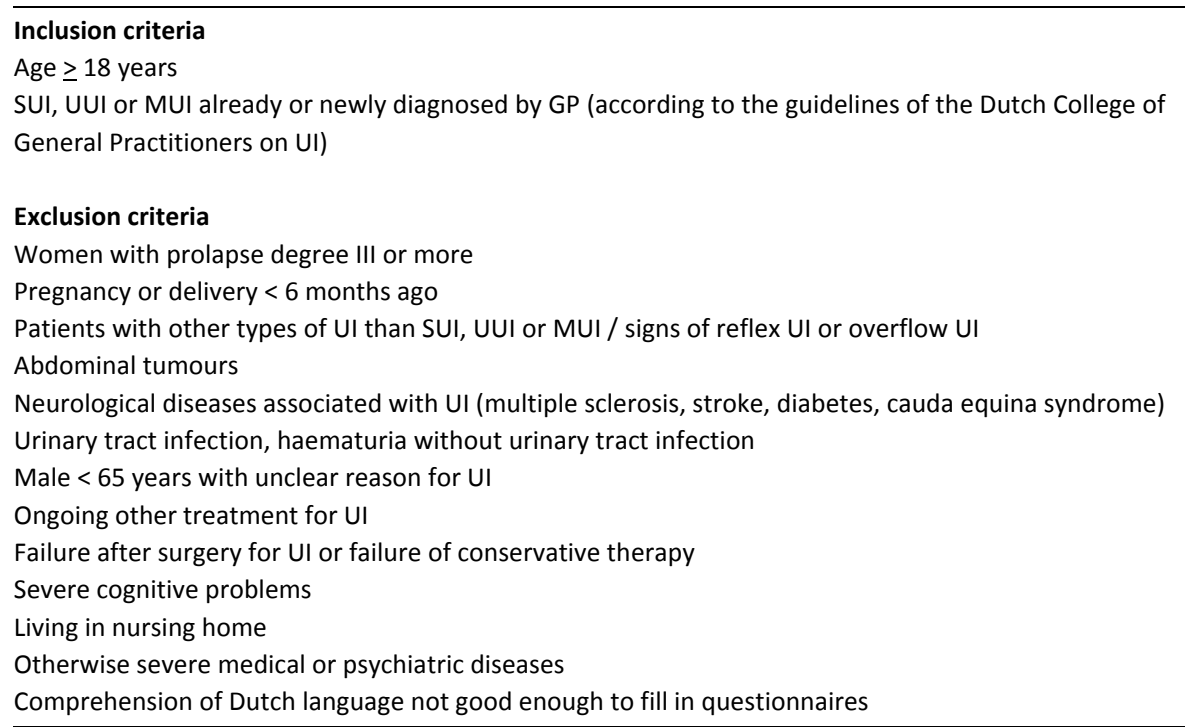

SUI, stress urinary incontinence; UUI, urgency urinary incontinence; MUI, mixed urinary incontinence; UI, urinary incontinence; GP, general practitioner. 


\section{Intervention}

Six nurse specialists provided the intervention (Figure 1). The nurse specialists received special training in tasks related to diagnostics, intervention and monitoring of incontinence based on guidelines and protocols and proved their competencies afterwards in an assessment. After the initial medical UI diagnosis by the GP, the nurse specialists further specified the diagnosis, registered problems in pelvic floor/bladder function (impairment), activities (disability), participation problems and the influence of personal and external factors following the International Classification of Functioning, Disability and Health (ICF). ${ }^{19}$ The nurses used micturition diaries and provided advice regarding lifestyle, toilet habits, bladder training and pelvic floor muscle training and, when appropriate, the choice of incontinence pads. Patients were treated during one year, with five to seven visits during the first three months, followed by consultations at six and 12 months to monitor effect and adherence. Details of the training of the nurse specialists and the intervention are described and free full text available elsewhere. ${ }^{17}$

\section{Care-as-usual}

Patients randomised to care-as-usual could not get a referral to the nurse specialist. We assumed that GPs would not change their care-as-usual, which is mostly restricted to pads prescription, where only a minority of UI patients gets active treatment or a referral to either physiotherapist or specialist. ${ }^{8,10,11,20}$

\section{Outcome measures}

The primary outcome was the International Consultation on Incontinence Questionnaire Short Form (ICIQ-UI SF) severity sum score of self-reported UI frequency, perceived UI quantity (weighted items) and the UI impact on health-related quality of life (Visual Analogue Scale [VAS]). ${ }^{18}$ Overall score ranges from 0 (no UI) to 21 (most severe UI). UI definitions followed International Continence Society (ICS) standards. $^{21}$

Effects were controlled for accepted baseline effect modifiers (conditions or risk factors that may influence the effects, such as age, UI type, parity, type of delivery, Body Mass Index [BMI], restricted mobility, anxiety/depression). ${ }^{1}$

The self-completed EuroQol health-related quality of life questionnaire (EQ-5D), provided a five dimensional descriptive profile of mobility, self-care, usual activities, pain/discomfort and anxiety/depression for general health status. ${ }^{22}$ Each dimension had three levels: no, some or severe problems. 

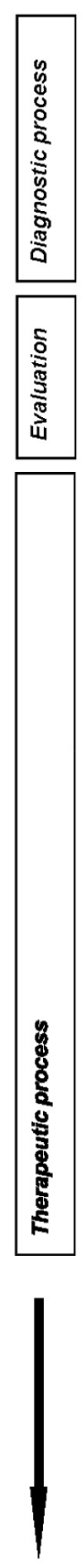

1. Input: - presumed diagnosis GP with relevant information - copy baseline data / measurements

2. Standardised history taking

3. Check bladder diary

1. Classifying type urinary incontinence: stress-, urgency- or mixed 2. Treatment plan

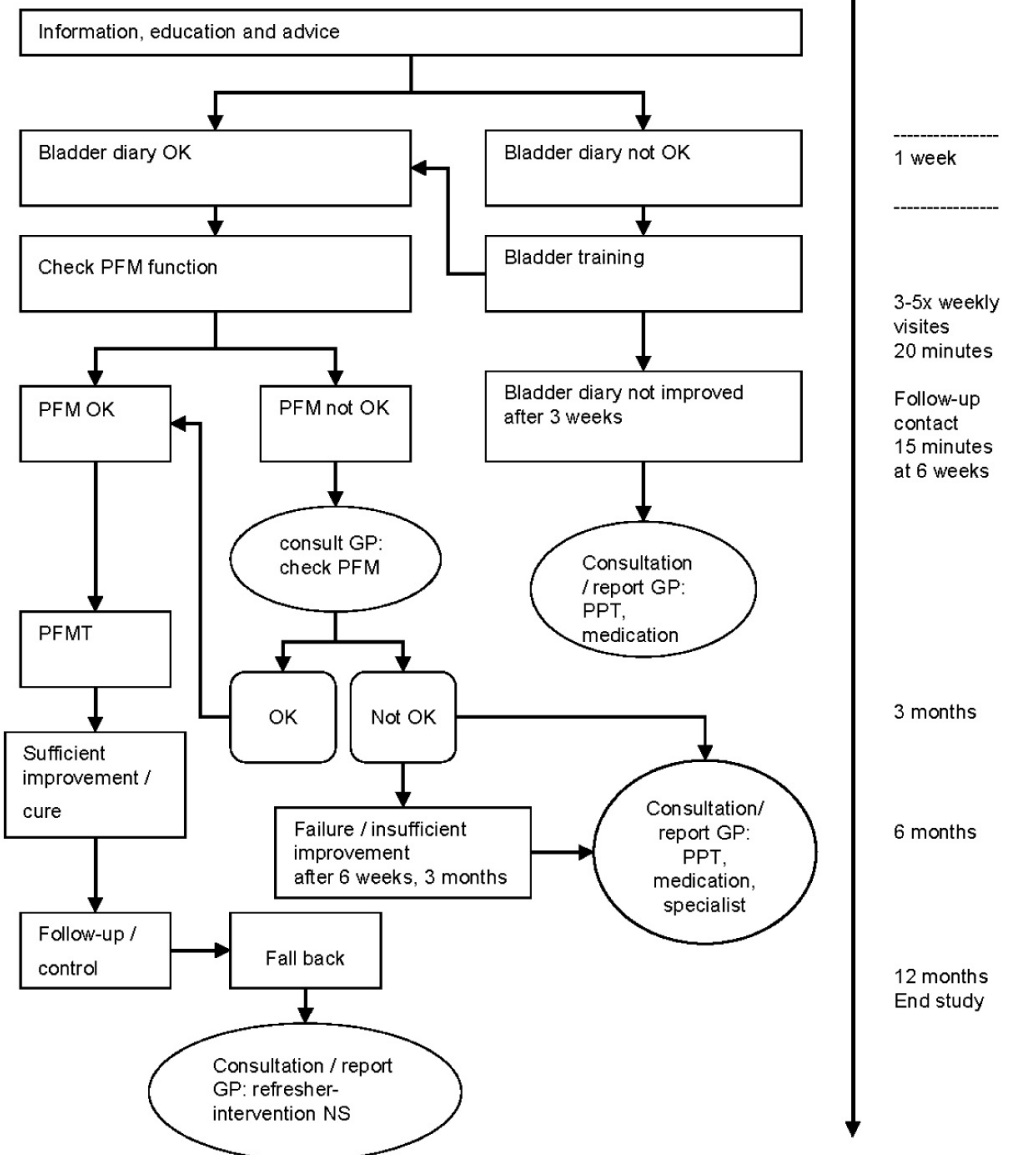

Figure 1 Flow chart intervention nurse specialist for patients with urinary incontinence.

PFM(T), Pelvic Floor Muscle (Training); GP, general practitioner; PPT, pelvic physiotherapist; NS, nurse specialist. Definitions following International Continence Society (ICS) standards. 


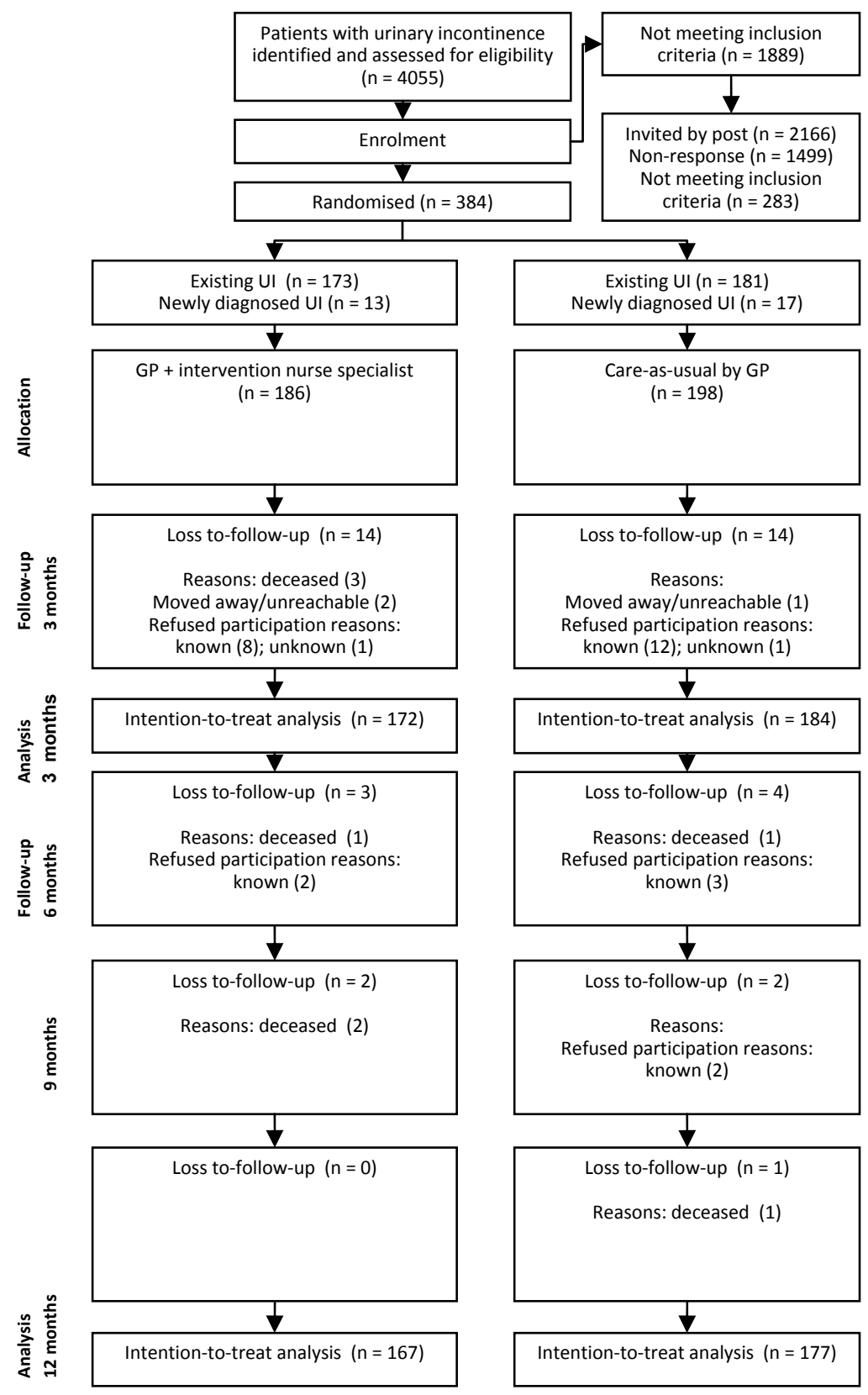

Figure 2 Flow of participants through each stage of the randomised trial and analysed for primary outcome. UI, UI; GP, general practitioner. 


\section{Data collection and statistical analysis}

Data were collected through postal questionnaires at baseline and for the two study end-points, three and 12 months. Non-responders were reminded by telephone two to three weeks later.

Kolmogorov-Smirnov tests were used to test normality of distributions in metric variables. Comparability of groups at baseline was checked for demographics, medical history, and general health. To test the overall effects in outcome at three months paired t-tests of differences with baseline were used by summing results over both groups. Repeated measures ANOVA was used to test outcome time differences from baseline between both groups, both at three months and for the duration of the total follow-up. Repeated measures ANCOVA was used to adjust for baseline outcome measurements, UI type, age, BMI, parity, complications at delivery and baseline EuroQol scales mobility and anxiety/depression. Dummy regression analysis was used on both the three months difference in outcome and on the linear-weighted trend over all outcome measurements in time. To test overall oneyear follow-up results for both groups a one-sample $t$-test was performed on the linear-weighted trend. Missing data were imputed by using the mean of observed scores for both groups. A p-value of $<0.05$ was assumed to be statistically significant. Data analysis was performed according to intention-to-treat principles, using SPSS-pc 15.0 and 16.0 (SPSS Inc., Chicago, IL, USA).

\section{Results}

A total of 109 GPs in 65 practices participated. They identified 4,055 UI patients. As expected because of the broad recruitment strategy, $47 \%(n=1889)$ had exclusion criteria (mostly co-morbidities) for the study. The remaining 2,166 patients were invited to participate. A total of 667 patients were interested and wished to be informed; 206 then refused and 77 were excluded due to co-morbidities.

Finally, 384 patients met the study criteria; 186 were randomly allocated to the intervention and 198 to care-as-usual (Figure 2).

Over the study period 40 (10.4\%) (19 intervention, 21 control) patients dropped out.

Both groups were comparable for factors such as age, gender, parity etc (Table 2). No separate analysis could be performed for gender as only very few male patients participated in the study.

Of the 186 patients randomised to the nurse specialist, 137 (73.6\%) had seven to nine consultations (mean time spent $3.1 \mathrm{~h}$ ). Fewer consultations were related to poor health $(n=14)$ or no motivation/too much burden $(n=15)$. Adverse events were unrelated to the nurse specialist intervention. 
Table 2 Demographics and baseline characteristics of patients ( $N=384$ [100\%]).

\begin{tabular}{|c|c|c|}
\hline Characteristic & $\begin{array}{l}\text { Intervention group } \\
\mathrm{n}=186(48.4 \%)\end{array}$ & $\begin{array}{l}\text { Usual care group } \\
\mathrm{n}=198(51.6 \%)\end{array}$ \\
\hline Female, n (\%) & $171(91.9)$ & $184(92.9)$ \\
\hline Age, mean $\pm s d$ & $64.5(14.1)$ & $64.9(11.6)$ \\
\hline \multirow[t]{2}{*}{$\mathrm{BMI}$, mean $\pm \mathrm{sd} \mathrm{kg} / \mathrm{m} 2$} & $28.3(6.5)$ & $28.1(5.8)$ \\
\hline & $6 m v$ & $7 m v$ \\
\hline \multicolumn{3}{|l|}{ Type UI, n (\%) } \\
\hline SUI & $68(36.6)$ & $67(34.4)$ \\
\hline UUI & $30(16.1)$ & $34(17.4)$ \\
\hline Mixed & $71(38.2)$ & $78(40.0)$ \\
\hline \multirow[t]{2}{*}{ NOS } & $17(9.1)$ & $16(8.2)$ \\
\hline & & $3 m v$ \\
\hline Prolapse problems, n (\%) & 24 (12.9) & $26(13.1)$ \\
\hline Constipation, n (\%) & $52(28.0)$ & $42(21.2)$ \\
\hline $\mathrm{FI}, \mathrm{n}(\%)$ & 33 (17.7) & $38(19.2)$ \\
\hline \multicolumn{3}{|l|}{ EuroQol 5-D: } \\
\hline \multicolumn{3}{|l|}{ Mobility (\%) } \\
\hline No problems & 59.1 & 57.6 \\
\hline Problems* & 40.9 & 42.4 \\
\hline \multicolumn{3}{|l|}{ Self-care (\%) } \\
\hline No problems & 89.2 & 93.4 \\
\hline Problems & 10.8 & 6.6 \\
\hline \multicolumn{3}{|l|}{ Usual activities (\%) } \\
\hline No problems & 69.4 & 61.1 \\
\hline Problems & 30.7 & 38.9 \\
\hline \multicolumn{3}{|l|}{ Pain/discomfort (\%) } \\
\hline No problems & 48.8 & 46.5 \\
\hline Problems & 55.8 & 53.5 \\
\hline \multicolumn{3}{|l|}{ Anxiety/depression (\%) } \\
\hline No problems & 71.5 & 74.2 \\
\hline Problems & 28.5 & 25.7 \\
\hline
\end{tabular}

Mv, missing value; BMI, body mass index, calculated as weight in kilograms divided by height in meters squared; UI, urinary incontinence; SUI, stress urinary incontinence; UUI, urgency urinary incontinence; MUI, mixed urinary incontinence; NOS, not otherwise specified; $\mathrm{FI}$, fecal incontinence. *Problems, sum of moderate and severe problems.

\section{Main results}

Overall, both the difference between both groups in ICIQ score at three months and the one-year linear trend was not significant ( $p=0.06$ and $p=0.15$ respectively) (Table 3). In contrast to our expectations, patients in both groups improved on the $\mathrm{ICIQ}$ score at three months and on the one-year linear trend (both end-points 
$p<0.001)$. However, when controlling for the accepted effect modifiers UI type, $\mathrm{BMI}$ and baseline ICIQ score we found that, compared with the care-as-usual group, the ICIQ score differences at three months significantly improved in the intervention group ( $B=-0.56, p=0.04 ; n=381$ ) (Table 3 ).

Neither BMI, nor UI type was significantly related to the one-year linear trend of the ICIQ score.

Table 3 Results intention-to-treat repeated measures ANCOVA/regression analysis on ICIQ-UI SF sum score; a higher score means more severity and impact of urinary incontinence.

\begin{tabular}{|c|c|c|c|c|c|c|}
\hline & $\begin{array}{l}\text { Intervention } \\
(n=186)\end{array}$ & $\begin{array}{l}\text { Care-as- } \\
\text { usual } \\
(n=198)\end{array}$ & $\begin{array}{l}\text { Between Group } \\
\text { Unstandardised B } \\
\text { Effect size (Cl 95\%) }\end{array}$ & p-value & $\begin{array}{l}\text { Overall } \\
\text { within Group } \\
\text { Effect size (Cl 95\%) }\end{array}$ & p-value \\
\hline \multicolumn{7}{|c|}{ Sum score ICIQ-UI SF (0-21) mean \pm SD (weighted frequency/volume [0-11] plus impact [0-10]) } \\
\hline baseline & $11.1 \pm 4.3$ & $11.3 \pm 3.7$ & & & & \\
\hline $3 \mathrm{mo}^{+}$ & $9.1 \pm 2.9$ & $9.7 \pm 2.9$ & $-0.51(-1.03$ to +0.01$)$ & 0.06 & $-1.79(-2.18$ to -1.41$)$ & $<0.001^{*}$ \\
\hline $3 \mathrm{mo}^{\ddagger}$ & & & $-0.56(-1.08$ to -0.04$)$ & $0.04 *$ & & \\
\hline $12 \mathrm{mo}^{+}$ & $9.6 \pm 3.7$ & $10.3 \pm 3.3$ & $-0.34(-0.80$ to +0.12$)$ & 0.15 & $-0.64(-0.93$ to -0.36$)$ & $<0.001^{*}$ \\
\hline $12 \mathrm{mo}^{\ddagger}$ & & & $-0.34(-0.81$ to +0.14$)$ & 0.16 & & \\
\hline $12 \mathrm{mo}^{\S}$ & & & $-0.63(-1.20$ to -0.06$)$ & $0.03 *$ & & \\
\hline
\end{tabular}

ICIQ-UI SF, International Consultation Incontinence Questionnaire Short Form; mo, month(s); BMI, body mass index, calculated as weight in kilograms divided by height in meters squared; typeUI, type of urinary incontinence. ${ }^{*}$ Statistically significant improvement ${ }^{\dagger}$ Model $=$ stimulus effect adjusted for baseline differences ${ }^{\ddagger}$ Model $=$ stimulus effect controlled for BMI, type UI, baseline sum score, and type $\mathrm{UI}$ *baseline sumscore $(\mathrm{n}=381)^{\S}$ Model = same model as under ${ }^{\ddagger}$, but for patients without baseline mental health problems anxiety/depression $(n=272)$.

\section{Relation with general health}

As suggested in the literature, we checked the influence of aspects of general health on improvements of the ICIQ sum scores, but found no influences, except for anxiety/depression. Although no differences after three months were found, after one year anxiety/depression was responsible for less improvement of the ICIQ sum score (interactive effect $B=1.02, p=0.03 ; n=381 ; B$ effect for patients without baseline anxiety/depression $=-0.63, p=0.03 ; n=272$ ) (Table 3$)$. 


\section{Discussion}

\section{Summary of main findings}

Significant differences between groups in favour of the intervention group were found after correction for known effect modifiers (UI type and BMI) after three months intervention. Surprisingly, both study groups improved in ICIQ sum score at three months and the one-year linear trend. Moreover, after one year of intervention, we found effects in specific situations (anxiety/depression). Before drawing any conclusion a few remarks must be made.

\section{Strengths and limitations of the study}

The choice for a pragmatic design ensured that the intervention was as close as possible to treatment options in daily practice. This will facilitate future implementation. We enrolled patients in our study who were typical for the normal GPcaseload. Nevertheless, in terms of external validity, our results may only be valid for patients with a more than mild UI or who are prepared to play an active role towards their UI. As already mentioned, our study population had a higher mean baseline ICIQ sum score (11) as compared with the mean sum score of seven for a primary care UI population as used in our power calculation. ${ }^{23}$ It is generally assumed that mild UI forms do profit most from bladder and pelvic floor muscle training. Our results, however, show that also UI patients with severe or complex UI can profit from this intervention. ${ }^{24-26}$ In patients with mild UI, the effects of our intervention may be higher.

The assumption prior to the study was that the care-as-usual group, as in many years before, would stay unchanged during the one-year study period. ${ }^{27,28}$ Given this assumption, the current recruitment numbers should have been sufficient to trace a significant difference. However, reality was different. Unexpectedly, the care-as-usual group also improved on the main outcome parameter. Consequently, the difference between both groups on the outcome became lower than expected. This would signify an unforeseen study effect that might be caused by several factors. GPs may have changed their care-as-usual policy, now being aware their UI approach is monitored (Hawthorne-effect). Theoretically, contamination might have occurred, but from our data we have no indication that this indeed occurred. A learning effect in GPs is in our view unlikely as the average GP included (only) six patients in the trial. Next to this, patients in the care-as-usual group may be influenced by being actively recruited for the study. An increased awareness about possible solutions for their UI problem may have occurred by extra contact with the GP for enrolment, the informed consent procedure and by repeatedly completing void- 
ing diaries and questionnaires. ${ }^{29,30}$ Finally, the general mean imputation of missing items for the intention-to-treat-analysis leads to a regression to the mean in effects.

Altogether, when any improvement in the care-as-usual group was to be factored in for the power calculation, more patients would have been needed. As a result, our findings may be quite conservative.

\section{Comparison with existing literature}

This is one of few pragmatic RCTs comparing nurse involvement for UI patients supplementary to care-as-usual by GPs with a long-term follow-up of one year. ${ }^{15,16}$ Our findings are in line with RCTs of nurse interventions for incontinent communitydwelling patients. ${ }^{15,16}$ However, comparability of results is limited due to varying populations, settings, outcome measurements, controls, nurse education level and duration of follow-up (often less than one year). Moreover, our finding that baseline reported anxiety/depression was associated with less UI improvement is in line with the literature. ${ }^{31-33}$ Lack of motivation, especially in those suffering from depression, might explain the lack of effect of our intervention on the one-year linear trend.

\section{Implications for clinical practice and future research}

$\mathrm{UI}$ is a chronic dynamic disorder and often complicated by co-morbidities. ${ }^{1}$ The positive effect of the nurse specialist intervention on the short-term would argue in favour of a repeated intervention by trained healthcare professionals to monitor the condition and achieve long-term effects. ${ }^{34}$

Further research should focus on tailoring the intervention to patients characteristics which are prognostic and predictive for UI and modifiable (depression 1,31,33, overweight ${ }^{1,35}$ ). Moreover, research into the cost-effectiveness of involving nurse specialists for UI patients in general practice is needed. ${ }^{36}$

\section{Acknowledgments}

We thank all participating GPs and Zorg Groep Zuid-Gelderland, Groene Kruis Domicura, Maastricht, AZIVO/ PCC of HAGA Hospital, The Hague, De Zorgboog, Elkerliek Hospital, Helmond, The Netherlands, their nurse specialists and the patients for their cooperation in the study. We thank Wendy Engering, Margriet Straver, Liesbeth van Hoef, Joyce Janssen and Nicole Castro for research assistance.

\section{Funding}

The study was approved and funded by the Netherlands Organisation for Health Research and Development (ZonMw), grant number 945-04-224. ZonMw was not involved in manuscript preparation and/or publication decisions. 
Ethical approval and Clinical Trial Registration Number

The study protocol was approved by the Medical Ethics Committees of all involved centres. Included patients gave their written informed consent. The trial is registered at http://www.controlled-trials.com/isrctn/62722772 and is reported following CONSORT guidelines for RCTs.

\section{What's known?}

Urinary incontinence (UI) primary care management is substandard, offering care rather than cure despite the existence of guidelines that help to improve cure. Main reasons for substandard care are lack of time of general practitioners and lack of targeted implementation strategies to adopt and practice guideline recommendations.

\section{What's new?}

Involving nurse specialists in care for $\mathrm{UI}$ patients supplementary to general practitioners can improve severity and impact of UI:

- Involving nurse specialists in UI primary care reduced severity and impact of UI after three months of intervention, after correction for effect modifiers; this is also the case in specific situations such as anxiety/depression. 


\section{References}

1. Milsom I, Altman D, Lapitan M et al. Committee 1. Epidemiology of Urinary (UI) and Faecal (FI) Incontinence and Pelvic Organ Prolapse (POP). In: Fourth International Consultation on Incontinence (ICI) July 5 - 8, 2008 Palais des Congres, Paris, France. Paris, France Health Publication Ltd 2009:63, 72, 89-92.

2. Abrams P, Cardozo L, Khoury S, Wein A. Fourth International Consultation on Incontinence (ICI) - July 5 - 8, 2008. Committee 1, 12. Palais des Congres, Paris, France. Paris, France Health Publication Ltd 2009.

3. Hay Smith J, Berghmans B, Burgio K et al. Committee 12. Adult Conservative Management. In: Fourth International Consultation on Incontinence (ICI) - July 5 - 8, 2008 Palais des Congres, Paris, France. Paris, France: Health Publication Ltd 2009:1079, 80; 94.

4. Wagg A, Cardozo L, Chapple C et al. Overactive Bladder and Continence Guidelines: implementation, inaction or frustration? Int JClin Pract. 2008;62(10):1588-93.

5. Kirby M, Artibani W, Cardozo L et al. Overactive bladder: the importance of new guidance. Int J Clin Pract. 2006;60(10):1263-71.

6. Monz B, Pons M, Hampel C et al. Patient-reported impact of urinary incontinence. Results from treatment seeking women in 14 European countries. Maturitas. 2005;30(52):suppl 2; 24-34.

7. Coyne K, Sexton C, Irwin D et al. The impact of overactive bladder, incontinence and other lower urinary tract symptoms on quality of life, work productivity, sexuality and emotional well-being in men and women: results from the EPIC study. BJU Int. 2008;101(11):1388-95.

8. Kirby M. Managing stress urinary incontinence - a primary care issue. Int J Clin Pract. 2006;60(2):184-9.

9. Shaw C, Atwell C, Wood F et al. A qualitative study of the assessment and treatment of incontinence in primary care. Fam Pract. 2007;24(5):461-7.

10. Albers-Heitner P, Berghmans B, Nieman F et al. Adherence to professional guidelines for patients with urinary incontinence by general practitioners: a cross-sectional study. J Eval Clin Pract. 2008; 14(5):807-11.

11. Albers-Heitner P, Berghmans B, Nieman F et al. How do patients with urinary incontinence perceive care given by their general practitioner? A cross-sectional study. Int J Clin Pract. 2008;62(3):508-15.

12. Staskin DR. The five 'Ps' of an incontinence clinical practice guideline. Int J Clin Pract. 2008;62(10): 1466-7.

13. Laurant $M$, Harmsen $M$, Faber $M$ et al. Revision of professional roles and quality improvement: a review of the evidence. London: The Health Foundation. 2010.

14. Voogdt-Pruis H, Beusmans G, Gorgels A et al. Effectiveness of nurse delivered cardiovascular risk management in primary care: a randomised trial. Br J Gen Pract 2010;60:40-6.

15. Du Moulin MFMT, Hamers JPH, Paulus A et al. The role of the nurse in community continence care: a systematic review. Int J Nurs Stud. 2005 5;42(4):479-92.

16. Du Moulin MFMT, Hamers JPH, Paulus A et al. Effects of Introducing a Specialized Nurse in the Care of Community-Dwelling Women Suffering From Urinary Incontinence: A Randomized Controlled Trial. J Wound Ostomy Continence Nurs. 2007;34(6):631-40.

17. Albers-Heitner $P$, Berghmans $B$, Joore $M$ et al. Study protocol. The effects of involving a nurse practitioner in primary care for adult patients with urinary incontinence: the PromoCon study (Promoting Continence). BMC Health Serv Res. 2008(8):84

18. Avery K, Donovan J, Peters T et al. ICl-Q: a brief and robust measure for evaluating the symptoms and impact of urinary incontinence. Neurourol Urodynam. 2004;23(4):322-30. http://www.iciq.net/ICIQ-Ulshortform.html (accessed 18-08-2010).

19. World Health Assembly Resolution 54.21.(2001) International Classification of Functioning, Disability and Health (ICF). http://www.who.int/classifications/icf/en/. [cited 2010 20-08].

20. Gerwen van M, Schellevis F, Lagro-Janssen T. Management of urinary incontinence in general practice: data from the Second Dutch National Survey. J Eval Clin Pract. 2009;15: 341-5. 
21. Haylen BT, De Ridder D, Freeman RM et al. An international urogynecological association (IUGA)/international continence society (ICS) joint report on the terminology for female pelvic floor dysfunction. Neurourol Urodynam. 2010;29(1):4-20.

22. Brooks R. EuroQol: the current state of play. Health Policy. 1996;37(1):53-72.

23. Abrams P, Cardozo L, Khoury S, Wein A. Incontinence Proceedings 2nd International Consultation on Incontinence - July 1, 2001- July 3, Paris. 2002:976.

24. Klovning A, Avery K, Sandvik H, Hunskaar S. Comparison of two questionnaires for assessing the severity of urinary incontinence: The ICIQ-UI SF versus the incontinence severity index. Neurourol Urodynam. 2009;28(5):411-5.

25. Shaw C, Brittain K, Tansey R, K. W. How people decide to seek health care: A qualitative study. Int J Nurs Stud. 2008;45(10):1516-24.

26. Dumoulin C, Hay-Smith J. Pelvic floor muscle training versus no treatment, or inactive control treatments, for urinary incontinence in women. Cochrane Database of Systematic Reviews 2010, (1): CD005654. DOI: 10.1002/14651858.CD005654.pub2.

27. McGrother $C$, Donaldson $M$, Shaw $C$ et al. Storage symptoms of the bladder: prevalence, incidence and need for services in the UK. BJU Int. 2004;93(6):763-9.

28. Wennberg A-L, Molander U, Fall M et al. A Longitudinal Population-based Survey of Urinary Incontinence, Overactive Bladder, and Other Lower Urinary Tract Symptoms in Women. Eur Urol. 2009;55(4):783-91.

29. Burgio KL, Goode PS, Locher JL et al. Behavioral Training With and Without Biofeedback in the Treatment of Urge Incontinence in Older Women: A Randomized Controlled Trial. JAMA. 2002;288(18):2293-9.

30. Melville JL, Katon W, Delaney K, Newton K. Urinary Incontinence in US Women: A Population-Based Study. Arch Intern Med. 2005;165(5):537-42.

31. Nygaard I, Turvey C, Burns TL et al. Urinary incontinence and depression in middle-aged United States women. Obstet Gynecol. 2003;101(1):149-56.

32. Gerwen van M, Schellevis F, Lagro-Janssen T. Comorbidities Associated with Urinary Incontinence: A Case-Control Study from the Second Dutch National Survey of General Practice. J Am Board Fam Med 2007;20:608-10.

33. Melville $\mathrm{JL}$, Fan $\mathrm{M}-\mathrm{Y}$, Rau $\mathrm{H}$ et al. Major depression and urinary incontinence in women: temporal associations in an epidemiologic sample. Am J Obstet \& Gynecol. 2009;201:490.e1-7.

34. Holroyd-Leduc JM, Straus SE. Comment on: Is there a role for estrogen in the prevention and treatment of urinary incontinence? CMAJ. 2005;172(8):1003-4.

35. Subak LL, Wing R, West DS et al. Weight loss to treat urinary incontinence in overweight and obese women. N Engl J Med. 2009;360(5):481-90.

36. Moore K, Wei Hu T, Subak L et al. Committee 22. Economics of Urinary and Faecal Incontinence, and Prolapse. In: Fourth International Consultation on Incontinence (ICI) - July 5 - 8, 2008 Palais des Congres, Paris, France. Paris, France Health Publication Ltd 2009:1708, 9. 



\section{Chapter 6}

Cost-effectiveness of involving nurse specialists for adult patients with urinary incontinence in primary care compared to care-as-usual:

An economic evaluation alongside a pragmatic randomised controlled trial

Pytha Albers-Heitner

Manuela Joore

Ron Winkens

Toine Lagro-Janssen

Johan Severens

Bary Berghmans

Neurourology and Urodynamics. Accepted for publication 08-07-2011. 


\section{Abstract}

Aims To determine the 12-month, societal cost-effectiveness of involving urinary incontinence (UI) nurse specialists in primary care compared to care-as-usual by general practitioners (GPs).

Methods From 2005 until 2008 an economic evaluation was performed alongside a pragmatic multicentre randomised controlled trial comparing UI patients receiving care by nurse specialists with patients receiving care-as-usual by GPs in the Netherlands.

186 adult patients with stress, urgency or mixed UI were randomly allocated to the intervention and 198 to care-as-usual; they were followed for one year.

Main outcome measures were Quality Adjusted Life Year ( $Q A L Y_{\text {societal }}$ ) based on societal preferences for health outcomes (EuroQol-5D), QALY patient based on patient preferences for health outcomes (EuroQol VAS), and Incontinence Severity weighted Life Year (ISLY) based on patient-reported severity and impact of UI (ICIQUI SF). Healthcare resource use, patient and family costs, and productivity costs were assessed. Data were collected by three-monthly questionnaires.

Incremental cost-effectiveness ratios were calculated. Uncertainty was assessed using bootstrap simulation and the expected value of perfect information was calculated (EVPI).

Results Compared to care-as-usual, nurse specialist involvement costs $€ 16,742$ / $\mathrm{QALY}_{\text {societal }}$ gained. Both $\mathrm{QALY}_{\text {patient }}$ and ISLY yield slightly more favourable costeffectiveness results. At a threshold of $€ 40,000 /$ QLY $_{\text {societal, }}$ the probability that the intervention is cost-effective is $58 \%$. The EVPI amounts to $€ 78$ million.

Conclusions Based on these results, we recommend adopting the nurse specialist intervention in primary care, while conducting more research through careful monitoring of the effectiveness and costs of the intervention in routine practice. 


\section{Introduction}

Urinary incontinence (UI) is a highly prevalent clinical condition and is well-known for its impact on general well-being and socio-economic burden. ${ }^{1}$ If not treated and monitored, $\mathrm{UI}$ is a chronic dynamic disorder, complicated by co-morbidities, with a strong tendency to worsen over time. ${ }^{1}$ In the Netherlands, $€ 160$ million a year is spent on incontinence pads. ${ }^{2}$ In view of the ageing population, a further increase is expected, with important implications for the future demand for incontinence services. National and international primary care UI guidelines indicate that for most UI patients, pelvic floor muscle and/or bladder training is the best non-invasive initial treatment in primary care to solve the problem. ${ }^{3}$ Despite these guidelines, however, training has so far not been consistingly offered. ${ }^{4-6}$ More than $50 \%$ of UI patients use incontinence pads, especially older people. ${ }^{4,6,7}$ This substandard and costly primary care management of $\mathrm{UI}$, offering care rather than cure, shows the need to improve UI treatment. One way to achieve this could be to involve incontinence nurse specialists. Recently, we compared a one-year intervention by trained nurse specialists with care-as-usual in adult UI patients in Dutch primary care. ${ }^{8,9}$ In this pragmatic multicentre randomised controlled trial (RCT) patients in both groups $(n=384)$ improved significantly in terms of UI severity and impact on health-related quality of life (HRQOL). After correction for effect modifiers (type of UI, Body Mass Index) we found significant differences in Ul severity and impact on HRQOL between groups in favour of the intervention group at three months $(n=186 ; p=0.04){ }^{1} \mathrm{~A}$ subgroup analysis at 12 months revealed that patients in the intervention group without self-reported anxiety/depression (effect modifier) at baseline had improved significantly more than those given care-as-usual $(p=0.03){ }^{1}$

However, little information is available on the cost-effectiveness of involving nurse specialists in UI primary care, compared to care-as-usual. ${ }^{10}$ The present economic evaluation aimed to determine the cost-effectiveness of involving nurse specialists in UI primary care compared to care-as-usual by general practitioners (GPs). The economic evaluation used both a societal and healthcare perspective and was conducted alongside the abovementioned RCT. ${ }^{8,9}$

\section{Materials and Methods}

\section{Study design}

From May 2005 until March 2008 we performed an economic evaluation alongside a pragmatic multicentre RCT comparing $\mathrm{UI}$ patients receiving nurse specialist care with $\mathrm{UI}$ patients receiving care-as-usual in general practice in four Dutch regions (Maastricht, Nijmegen, Helmond, The Hague). The study protocol and clinical results 
have been reported elsewhere. ${ }^{8,9}$ Briefly, adult patients with stress, urgency or mixed UI, already known as such or newly diagnosed by their GP and meeting the inclusion criteria, were eligible and actively recruited by their GP to participate in the study, and were followed for one year. After written informed consent, randomisation was computer-generated, with allocation concealment by sealed envelopes. Based on a mean UI severity sum score of 7.18 (SD 6.64) on the International Consultation on Incontinence Questionnaire Short Form (ICIQ-UI SF) ${ }^{11}$, as well as an expected clinically important improvement of two points on the outcome scale (delta value $2 / 6.64=0.301$ ), a power of $80 \%$ and a two-sided significance level of 0.05 , we needed 175 patients per arm, 350 in total. Data were collected through postal questionnaires at baseline, and three, six, nine and 12 months. Nonresponders were reminded by telephone two to three weeks later. The study protocol was approved by the Medical Ethics Committees of all participating medical centres.

\section{Intervention}

Six nurse specialists who had received specialised training and a competencies assessment provided the intervention. After initial medical UI diagnosis by a GP, the nurse specialists further specified the diagnosis. The nurses asked patients to complete micturition diaries and advised them on lifestyle, toileting habits, bladder- and pelvic floor muscle training and, when appropriate, the choice of incontinence pads. Patients were treated for one year, with five to seven visits during the first three months, followed by consultations at six and 12 months to monitor effect and adherence.

\section{Care-as-usual}

Patients randomised to care-as-usual were not referred to the nurse specialist. We assumed that GPs would not change their care-as-usual. ${ }^{5,6}$

\section{Outcomes}

Three effect measures were used: Quality Adjusted Life Year based on societal preferences for health outcomes $\left(\mathrm{QALY}_{\text {societal }}\right){ }^{12}$ Quality Adjusted Life Year based on patient's preferences for health outcomes $\left(Q A L Y_{\text {patient }}\right){ }^{12}$ and the newly developed Incontinence Severity weighted Life Year (ISLY) based on patient-reported UI severity and impact as assessed with the ICIQ-UI SF. ${ }^{11}$ The self-completed EuroQol quality of life questionnaire, ${ }^{12}$ provides a five-dimension descriptive profile for general health status (mobility, self-care, usual activities, pain/discomfort and anxiety/depression) and a Visual Analogue Scale (VAS) ranging from worst (0) to best 


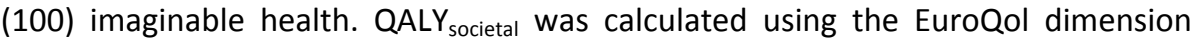
scores to obtain quality of life weights, following the algorithm developed by Dolan et al. ${ }^{13}$ Quality of life weights for the $Q A L Y_{\text {patient }}$ calculation were obtained by dividing the EuroQol VAS scores by 100. Since generic HRQOL may lack responsiveness in UI, the ISLY, based on the validated and widely applied ICIQ-UI SF, was developed as a disease specific outcome. ${ }^{11}$ The ICIQ yields one severity sum score for selfreported UI frequency and quantity (weighted items) and a VAS score for the UI impact on quality of life. The ICIQ score ranges from zero (no UI) to 21 (most severe UI). The UI severity weights that were needed to calculate the ISLY were obtained by linear transformation of the ICIQ sum scores to a zero (worst state of incontinence) to one (no incontinence) scale. The ISLY score is calculated as follows:

\section{1 - ICIQ-UI sum score / 21 (= maximum ICIQ - UI sum score)}

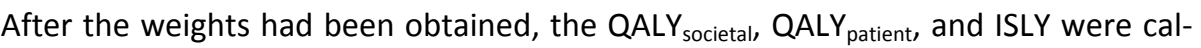
culated using the area-under-the-curve (AUC) method for each individual patient ${ }^{14}$ :

$$
A U C_{i}=\frac{Y_{i, 0}+Y_{i, 1}}{2} *\left(t_{1}-t_{0}\right)+\frac{Y_{i, 1}+Y_{i, 2}}{2} *\left(t_{2}-t_{1}\right)+\frac{Y_{i, 2}+Y_{i, 3}}{2} *\left(t_{3}-t_{2}\right)+\frac{Y_{i, 3}+Y_{i, 4}}{2} *\left(t_{4}-t_{3}\right)
$$

where $Y_{x}$ is the weight per data collection point, and $t$ is the data collection point. Patients who died during the study were given a weight of zero at death.

\section{Costs}

The Dutch Healthcare Insurance Board's manual for cost research was applied for the identification, measurement and evaluation of costs. ${ }^{15}$ The manual has been issued to complement the Dutch guidelines for pharmacoeconomic research, ${ }^{16}$ which are used in designing, conducting, and reporting economic evaluations. The societal perspective indicates that all costs were taken into account, regardless of who pays for them. ${ }^{17}$ Healthcare resource use, patient and family costs, and productivity costs were collected using self-administered three-monthly retrospective questionnaires. They included the costs of GP, medical specialist, and physiotherapist treatment, hospital admittance, incontinence pads and medication. Patient and family costs comprised travel costs and out-of-pocket costs for incontinence pads, medication, and other means or aids. The PROductivity and DISease Questionnaire (PRODISQ) was used to measure absence from paid and unpaid work. ${ }^{18}$ Costs associated with productivity loss were calculated using the friction costs method. ${ }^{18}$ The costs of the nurse specialist intervention were based on the standard unit price for home care nursing ${ }^{15}$ and patient-level recording of contact hours on a Case Record Form. Standard unit prices were derived from the abovementioned manual for cost 
research. ${ }^{15}$ Shadow prices were used for household or other unpaid activities. ${ }^{15}$ If no standard unit prices were available, market prices were retrieved. Costs were calculated by multiplying the volumes of healthcare by the corresponding unit prices. Total societal costs were calculated by adding up total healthcare costs, patient and family costs and productivity costs. All costs are reported in Euros at the 2007 price level.

\section{Analysis}

Data analysis was based on intention-to-treat principles. Comparability of groups at baseline was checked in terms of demographics, medical history and general health. Missing data were imputed using the mean of observed scores for both groups. In this analysis, the cost-effectiveness of the nurse specialist intervention versus careas-usual was assessed by relating the incremental societal or healthcare costs of the two approaches to the incremental outcomes in terms of generic HRQOL from the societal perspective $\left(\mathrm{QALY}_{\text {societal }}\right)$, the patient's perspective $\left(\mathrm{QALY} \mathrm{Y}_{\text {patient }}\right)$ and diseasespecific severity and impact (ISLY). The time horizon of the analysis was one year, so discounting was irrelevant. ${ }^{17}$ A regression-based adjustment was used to correct for baseline differences in EuroQol utility scores, EuroQol VAS scores and ICIQ-UI weighted scores after checking the normality of residuals. ${ }^{19}$ Means with $95 \%$ confidence intervals $(\mathrm{Cl})$ for costs were determined. The uncertainty in the incremental costs, outcomes and cost-effectiveness ratios (ICERs) was evaluated using bootstrap simulation. ${ }^{20}$ Bootstrap simulation is a non-parametric method in which cost and effectiveness pairs are randomly drawn, with replacement, from the data, to obtain a sample equal to the size of the original study sample. This is repeated 1,000 times, in order to get 1,000 estimates of the incremental costs and effects. The results of the simulation are shown in a cost-effectiveness plane. Bootstrapped confidence intervals $(\mathrm{BCl})$ for incremental costs and outcomes were determined by taking the 2.5th and 97.5th percentile. The probability that a new treatment is cost-effective depends on what society is prepared to pay per unit of gain in effectiveness, the socalled ceiling ratio. ${ }^{21}$ The net monetary benefit for different ceiling ratios was calculated for the nurse specialist intervention and for care-as-usual by subtracting the incremental costs from the incremental effects, multiplied by the ceiling ratio. Based on the bootstrap simulations, cost-effectiveness acceptability curves (CEACs) show the probability that an intervention has the highest net monetary benefit, and is thus deemed cost-effective, given different ceiling ratios. ${ }^{22}$ Due to stochastic uncertainty, however, there is always a chance that the 'wrong' decision will be made. We estimated the upper bound on the value of further research to reduce uncertainty by performing a value-of-information analysis (VOI). The expected costs of uncertainty can be interpreted as the expected monetary value of perfect information (EVPI), as perfect information can eliminate the risk of making the wrong deci- 
sion. We calculated the EVPI by subtracting the net monetary benefit of the intervention we would choose under conditions of uncertainty, from the net monetary benefit of the optimal decision we would make if there was no uncertainty. ${ }^{23}$ The total EVPI for the Netherlands, was obtained by multiplying the EVPI with the population that would be affected by the decision over the next 5 years ( $N=250,014$ new patients). This was based on an annual incidence of 10 new UI patients per GP practice and 5,400 GP practices in the Netherlands. ${ }^{24}$ Finally, this population was discounted at a $4 \%$ rate. $^{15}$

\section{Sensitivity and subgroup analyses}

In a one-way sensitivity analysis, we varied the intervention costs (unit price for a practice nurse instead of home care nursing). Nurse specialist salary costs in the intervention were valued based on hourly home care nursing rates ( $€ 62.32$ ) in the base case analysis. This rate includes time and travel costs for home care visits. In our study home visits occurred in only $50 \%$ of all contacts. Therefore, we performed a sensitivity analysis based on the $€ 23.71$ per hour rate for practice nurses, who do most of their work in a GP setting. The time horizon of the analysis was varied by only considering the treatment during the first three months, which covered the most frequent consultations and most of the treatment effect (a three months time horizon versus one year). Finally, since ancillary analysis showed that controlling for baseline reported anxiety/depression, was significantly related to lower clinical improvement, a post-hoc subgroup analysis was performed on groups reporting anxiety/depression. .,9 $^{8}$

\section{Results}

384 Patients met the inclusion criteria; 186 were randomly allocated to the intervention and 198 to care-as-usual. ${ }^{8,9}$ The population predominantly consisted of women (93\%), with a mean age of 65 and the groups were comparable. ${ }^{8,9}$ of the 186 patients randomised to the nurse specialist, 137 (73.6\%) were treated according to protocol (seven to nine consultations). Fewer consultations than prescribed by the protocol were related to poor health $(n=14)$ or no motivation/too much burden $(n=15)$. There were no adverse events related to the nurse intervention.

Most patients reported no problems on the dimensions of the EuroQol. ${ }^{8,9}$ One out of four patients reported severe anxiety/depression problems. Patients in both groups had improved significantly in each EuroQol dimension and on the VAS, after three months as well as after one year (all $p<0.001$ ). There were no statistically significant differences in change scores between groups for the EuroQol dimensions or on the VAS, after correction for baseline differences (Figure 1a, b). 

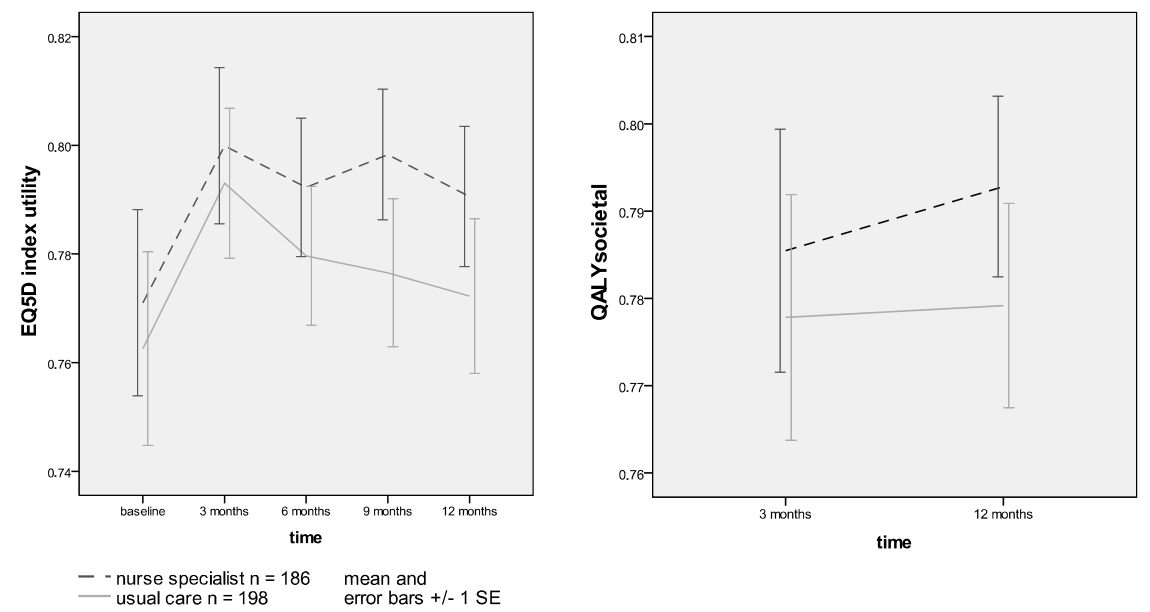

Figure 1a Values of EQ-5D index utilities yielding Quality Adjusted Life Year from the societal perspective (QALYsocietal) (mean, standard error [SE]).

Patients in both groups had improved significantly for each EuroQol dimension after three months and after one year (all $p<0.001$ ).
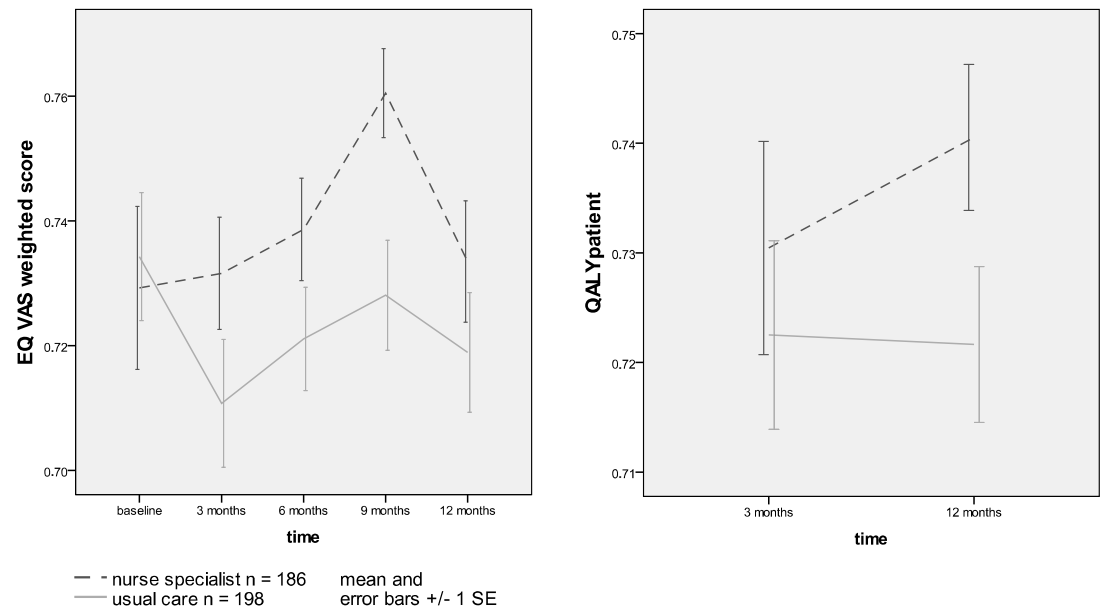

Figure 1b EuroQol VAS weighted values yielding Quality Adjusted Life Year from the patient's perspective (QALYpatient) (mean, standard error [SE]).

Patients in both groups had improved significantly on the EuroQol VAS after three months and after one year (all $p<0.001)$.

The incremental QALY societal and QALY patient in favour of the intervention group were 0.01 (95\% BCl: -0.05 to 0.08 ) and 0.02 ( $95 \% \mathrm{BCl}:-0.02$ - 0.06) respectively (corresponding to 4.9 and 7.2 days in best imaginable health) (Table I). Patients in both groups improved significantly in terms of ICIQ-UI scores $(p<0.001)$ (Figure 1c). Statistically significant differences in change scores between groups were found at 

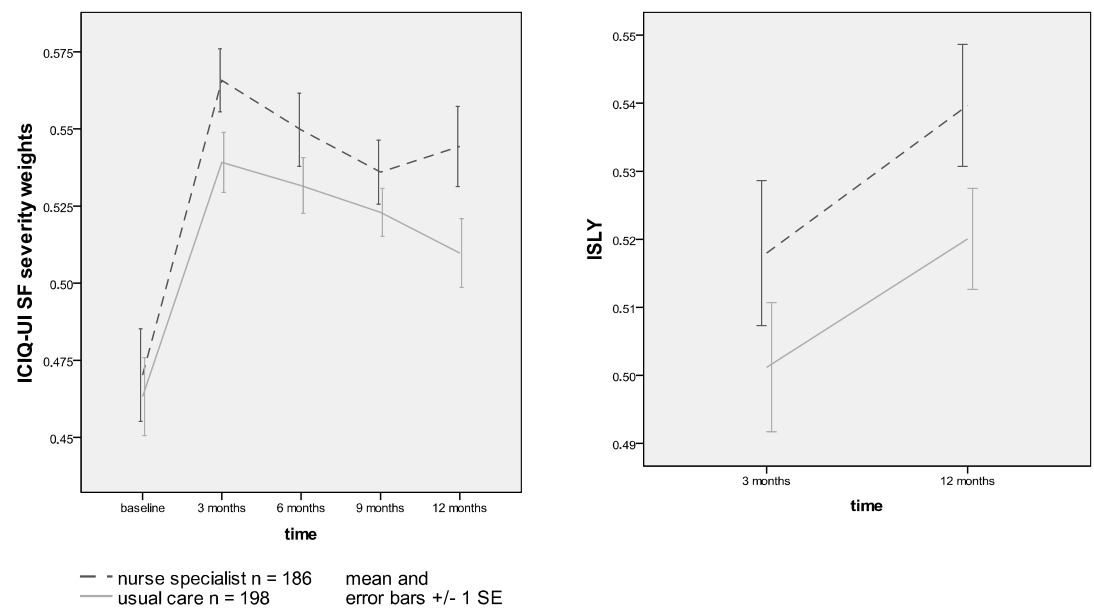

Figure 1c Values of transformed sum score of the ICIQ-UI SF yielding Incontinence Severity weighted Life Year (ISLY) (mean, standard error [SE]).

Patients in both groups had improved significantly on the ICIQ-UI SF after three months and after one year (all $p<0.001$ ). Statistically significant differences in change scores between groups were found at three months after correction for effect modifiers $(p=0.04)$ and in patients in the intervention group without self-reported anxiety/depression at baseline after one year $(p=0.03)$.

three months, after correction for effect modifiers $(p=0.04)$ and in patients in the intervention group without self-reported anxiety/depression at baseline after one year $(p=0.03){ }^{8,9}$ The difference in ISLY in favour of the intervention group was 0.02 (95\% BCl: -0.03 to 0.07 ) (the equivalent of 7.08 days without UI) (Table I).

\section{Costs}

Healthcare costs, patient and family costs, and productivity costs are summarised in Tables I and II, listing prices per item and volumes of medical care consumed. The mean healthcare costs amounted to $€ 618$ in the intervention group, and $€ 398$ in the control group (incremental costs $€ 220 ; 95 \% \mathrm{BCl}$ € 99 to $€ 369$ ). The largest proportions of these costs consisted of the costs of incontinence pads and intervention costs. The difference in healthcare costs between the groups was mainly due to the intervention costs. Mean healthcare costs for the intervention were $€ 195$ (95\% BCI: $€ 169$ - $€ 224$ ) (Table II). Patient and family costs (out-of-pocket costs and travel costs) were low in both groups. There was no productivity loss from paid work and costs due to absence from unpaid work were also low in both groups. Total societal costs amounted to $€ 677$ in the intervention group, and $€ 453$ in the control group (incremental costs $€ 224 ; 95 \% \mathrm{BCl}$ € $80-€ 422$ ). 


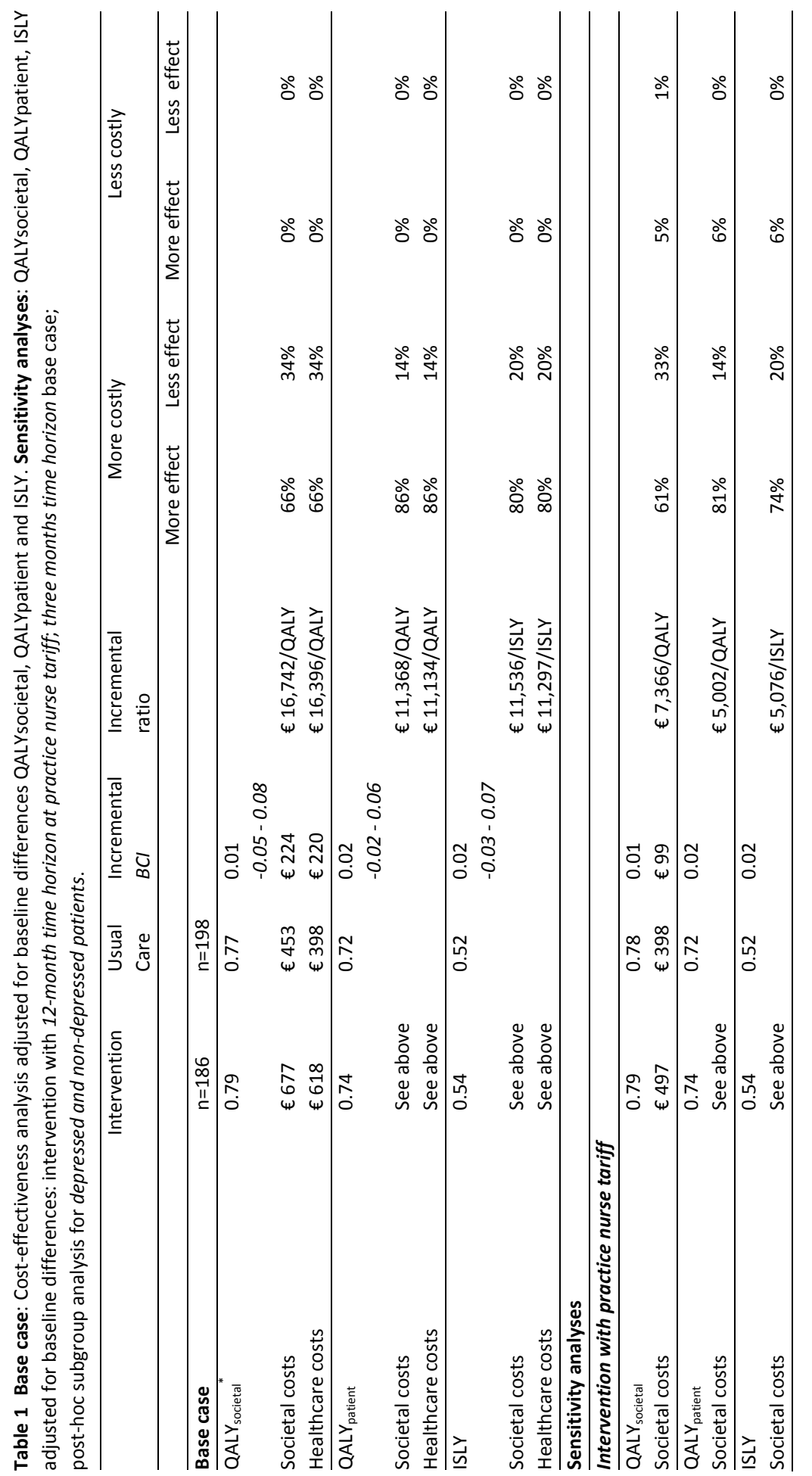




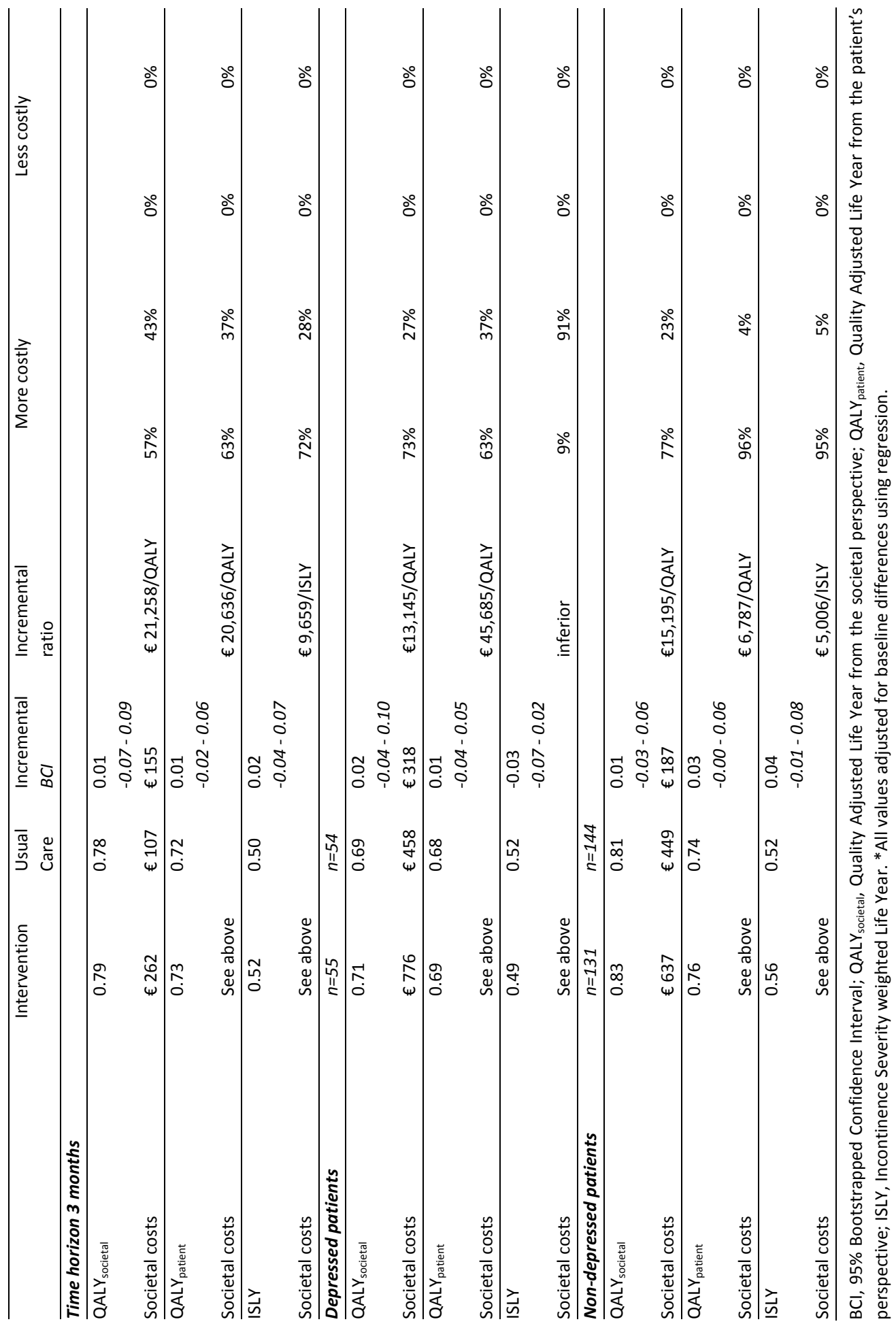




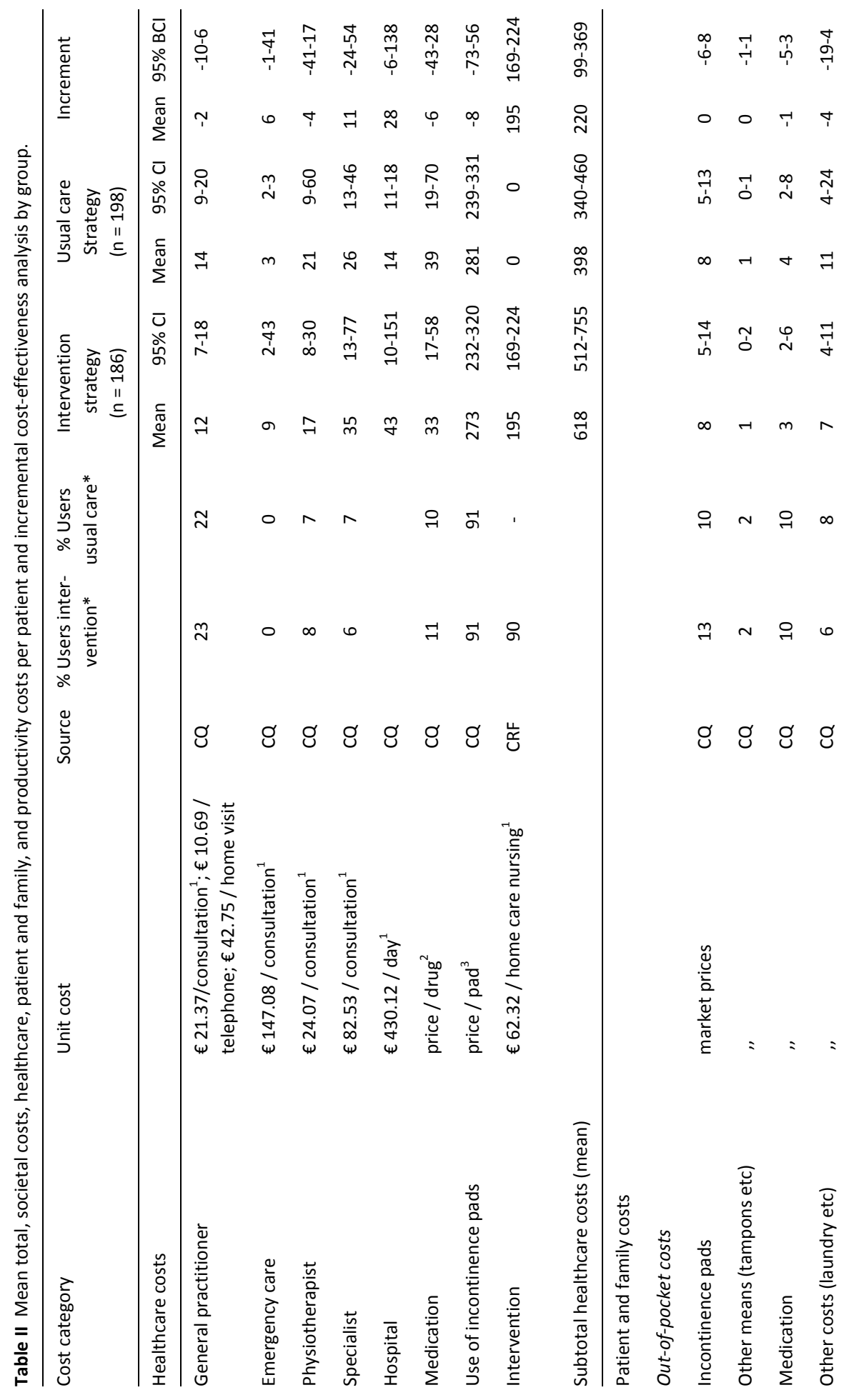




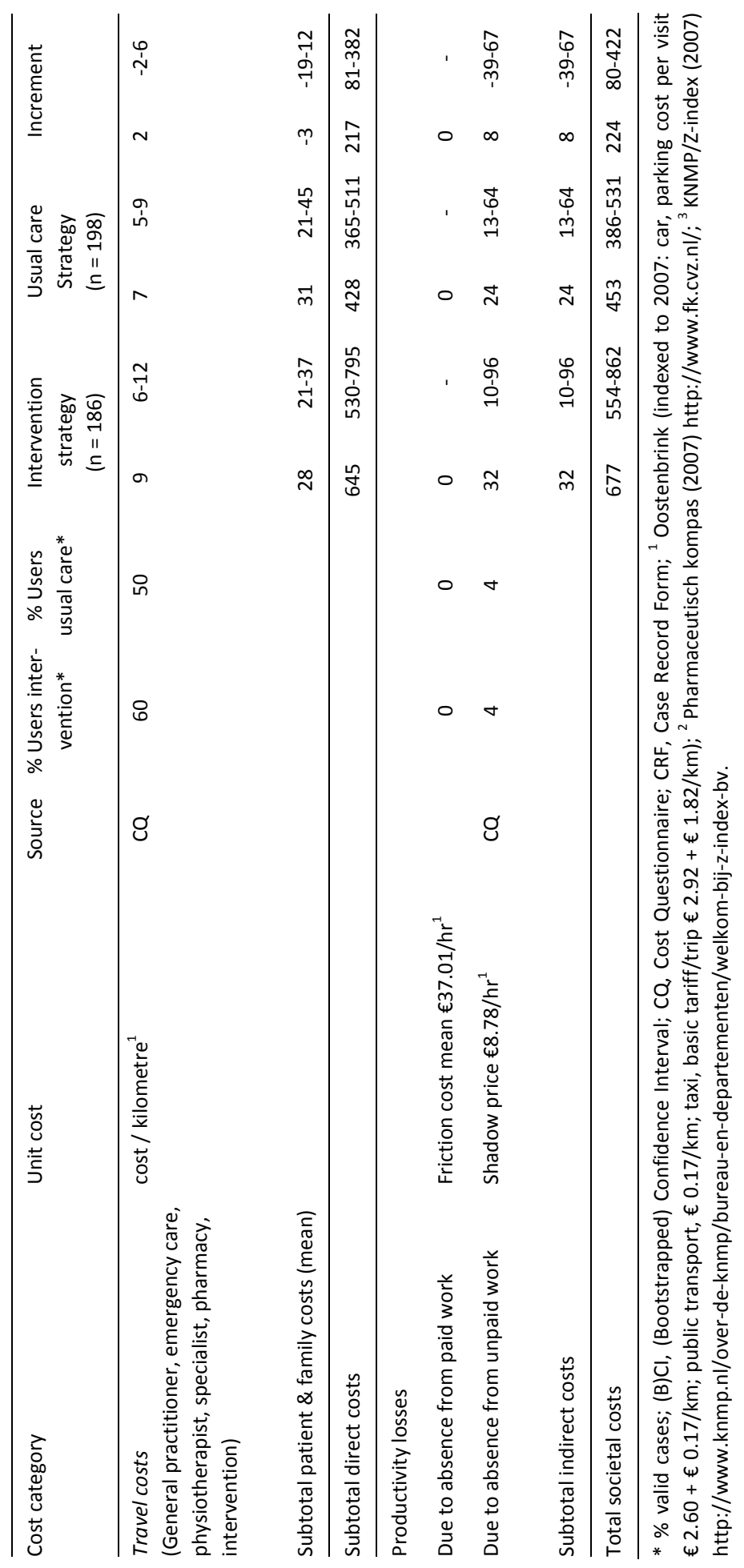




\section{Cost-effectiveness analyses}

Based on the results of the bootstrap simulation (Figure 2a), the cost-effectiveness ratio from the societal perspective was $€ 16,742$ per $Q A L Y_{\text {societal }}$ gained (Table I). The cost-effectiveness ratio from the healthcare perspective was slightly lower: $€ 16,396$ per $Q A L Y_{\text {societal }}$ gained. The corresponding values from a patient perspective were $€ 11,368$ and $€ 11,134$, respectively, per QALY patient gained. The costs per ISLY gained amounted to $€ 11,536$ and $€ 11,297$, respectively (Table I). At a ceiling ratio of $€ 40,000$ per QALY $_{\text {societal, }}$ the probability that the intervention is cost-effective, compared to care-as-usual, was $58 \%$ based on $Q A L Y_{\text {societal. }}$ Using the same threshold as a proxy for $Q A L Y_{\text {patient }}$ resulted in a probability of $77 \%$, based on $Q A L Y_{\text {patient }}$ (Figure $2 b$ ).

Figure $2 \mathrm{~b}$ also shows the relationship between a threshold for a life year without $\mathrm{UI}$ and the probability that the intervention is cost-effective.

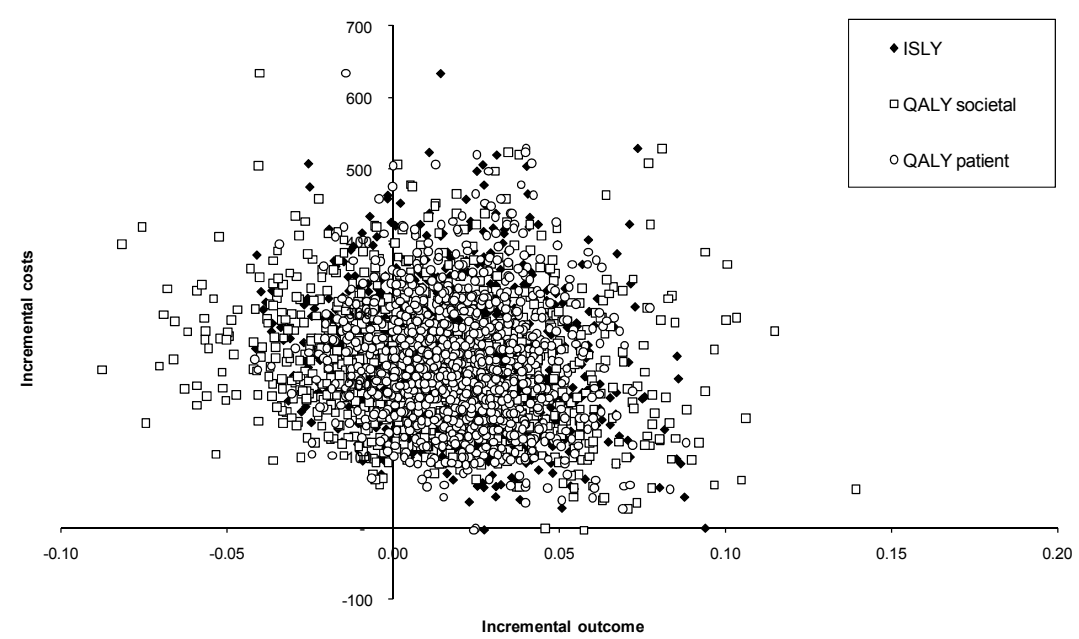

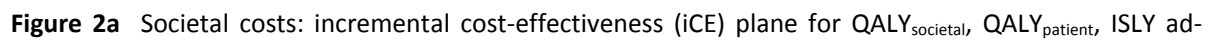
justed for baseline differences. Bootstrap replications $(n=1,000)$ of the mean difference in costs and outcomes generated from trial data. The majority of the bootstrap results are in the north-east quadrant, indicating that the intervention is more expensive and more effective than care-as-usual. 


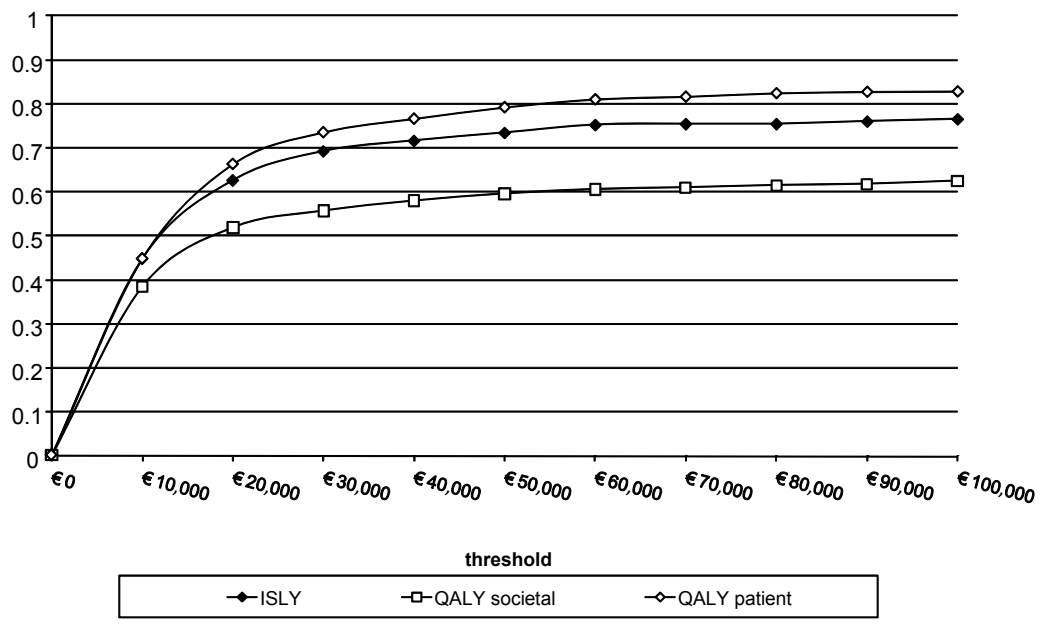

Figure 2b Cost-effectiveness acceptability curves (CEACC), Quality Adjusted Life Year from the societal perspective ( $\left.Q A L Y_{\text {societal }}\right)$, Quality Adjusted Life Year from the patient's perspective QALY patient, Incontinence Severity weighted Life Year (ISLY) adjusted for baseline differences for nurse specialist care (societal costs).

\section{Sensitivity analyses and subgroup analyses}

The results of the sensitivity and subgroup analyses are listed in Table I. The sensitivity analysis showed that the ICERs became lower when the costs of the intervention were based on a practice nurse instead of a nurse specialist. A three-month instead of 12-month time horizon resulted in higher incremental cost/QALY outcomes, but lower incremental cost/ISLY. The subgroup of patients without anxiety/depression complaints had lower incremental societal costs ( $€ 187$ ), with lower incremental $Q A L Y_{\text {societal, but higher incremental }} \mathrm{QALY}_{\text {patient }}$ and ISLY. At a ceiling ratio of $€ 40,000$, the probability that the intervention for the subgroup of patients without anxiety/depression is cost-effective compared to care-as-usual increased to $69 \%$ $(\mathrm{QALY} \mathrm{societal})$ and $88 \%\left(\mathrm{QALY}_{\text {patient }}\right)$, respectively.

\section{Expected value of perfect information}

The uncertainty surrounding the decision whether or not to adopt nurse specialist involvement resulted in an EVPI of $€ 311$ per patient, based on QALY societal. Given a ceiling ratio of $€ 40,000$ and based on 250,014 new patients consulting Dutch GP's with $\mathrm{UI}$ in the next five years, the population EVPI is $€ 78$ million. 


\section{Discussion}

Compared to care-as-usual, the involvement of nurse specialists costs $€ 16,742$ per $\mathrm{QALY}_{\text {societal }}$ gained. Using $\mathrm{QALY}$ patient or ISLY results in slightly more favourable costeffectiveness outcomes. Based on a threshold of $€ 40,000,{ }^{25}$ which is commonly applied in the Netherlands, involving nurse specialists in UI primary care has an acceptable cost-effectiveness ratio, though with a degree of uncertainty. The value of perfect information in the Netherlands amounts to $€ 78$ million, representing the upper bound on the value of further research to inform the decision to adopt nurse specialist involvement in the management of UI in primary care. Using QALY patient and the ISLY as effect measures results in slightly lower cost-effectiveness ratios with less uncertainty. The ICERs are considerably lower when the costs of the intervention are based on practice nurses instead of nurse specialists. The subgroup analysis shows that for UI patients without reported anxiety/depression, the probability that the nurse specialist intervention is cost-effective at a ceiling ratio of $€ 40,000$ as compared to care-as-usual is considerably higher than for depressed patients ( $69 \%$ instead of $58 \%$ based on QALY societal).

To our knowledge, this is the first full economic evaluation alongside a pragmatic RCT for conservative UI management in primary care both from the societal and the patient's perspective for conservative management in primary care. ${ }^{10}$ The few available cost-effectiveness analyses of conservative UI management are incomparable with our results because of differences in methodology, target population and programme aspects. ${ }^{10}$ Our VOI analysis offers guidance for coverage decisions. ${ }^{26}$

Since generic HRQOL from the patient's perspective may lack responsiveness in UI, the ISLY was developed as a sensitive and disease specific addition to the QALYs. Interpretating the costs per ISLY gained is not yet possible. It would be valuable to have an indication of the societal threshold for the costs of a UI-free life year as measured by ISLY. This issue deserves further research. For comparability across UIstudies, we would recommend that other researchers in this field use the ISLY as an outcome in economic evaluations.

Unexpectedly, the care-as-usual group also improved in terms of the main outcome parameter, making group differences smaller, which might have been caused for several reasons. ${ }^{8,9}$ In short, less than one in five UI patients, mostly female and already diagnosed, finally participated and most of the included patients had a longer history of $\mathrm{UI}$, and incident cases may have been underrepresented. ${ }^{9}$ Our active recruitment may have made patients in the control arm aware of the severity of their condition and the treatments available. Furthermore, although our data give no indication of this, ${ }^{9}$ randomisation at patient level may have caused contamination. ${ }^{8}$

Besides, since some consequences of treatment may take considerable time to manifest themselves, the follow-up of the trial may have been too short to capture 
all benefits such as changes in patients' lifestyle, use of pads and healthcare services provided (e.g. operations prevented). ${ }^{27}$

Thus, although we consider our trial to be pragmatic based on the CONSORT statement criteria and its extension for pragmatic trials, ${ }^{28-30}$ a more ideal trial should have used naturalistic patient recruitment, a longer follow-up, and a larger and representative sample of UI caregivers.

Our study methods can serve as a model for future cross-comparisons of both generic $H R Q O L$ from the societal perspective $\left(Q A L Y_{\text {societal }}\right)$ for coverage decisions and HRQOL from the patient's perspective ( $\left.Q A L Y_{\text {patient }}\right)$, along with disease specific outcomes (ISLY), for clinical decision making. We recommend further research on the ISLY, based on the widely applied ICIQ-UI SF. Adding screening, evaluation and adequate treatment of anxiety/depression to diagnosing UI can improve costeffectiveness. $^{31}$

\section{Conclusion}

Based on our cost-effectiveness results and taking into account that the implementation costs are likely to be minor, we recommend adopting the nurse specialist intervention in primary care, while conducting further research. Careful monitoring of the performance of the intervention with suitable patient registries of real world data allow for longer follow-up with future evidence development and will yield further information for coverage decisions.

\section{Acknowledgement}

We are grateful to the nurse specialists and the patients who took part in this study. We want to thank Zorg Groep Zuid-Gelderland; Groene Kruis Domicura, Maastricht; AZIVO/PCC at Leyenburg Hospital, The Hague; De Zorgboog, Quartz of the Elkerliek Hospital, Helmond, The Netherlands, for their participation. A debt of gratitude is also owed to all participating GPs. Many thanks to Wendy Engering, Margriet Straver and Liesbeth van Hoef for research assistance, to Joyce Janssen for data base handling and to Nicole Castro for data entry. 


\section{References}

1. Milsom I, Altman D, Lapitan M, Nelson R, Sillén N, Thom D. Committee 1. Epidemiology of Urinary (UI) and Faecal (FI) Incontinence and Pelvic Organ Prolapse (POP). In: Abrams P, Cardozo L, Khoury S, Wein A, editors. Incontinence 4th International Consultation on Incontinence Paris July 5-8, 2008. Plymouth: Health Publication Ltd; 2009. p. 63, 72, 89-92.

2. Health Insurance Board (CVZ). GIP databank genees- en hulpmiddelen. 2011 [updated 20-09-2010; cited 20-01-2011]; Available from: http://www.gipdatabank.nl/.

3. Hay-Smith J, Berghmans B, Burgio K, Dumoulin C, Hagen S, Moore K, et al. Committee 12. Adult Conservative Management. In: Abrams P, Cardozo L, Khoury S, Wein A, editors. Incontinence 4th International Consultation on Incontinence Paris July 5-8, 2008. Plymouth: Health Publication Ltd; 2009. p. 1079, 80; 94.

4. Monz B, Pons M, Hampel C, Hunskaar S, Quail D, Samsioe G, et al. Patient-reported impact of urinary incontinence. Results from treatment seeking women in 14 European countries. Maturitas. 2005;30(52):suppl 2; 24-34.

5. Albers-Heitner P, Berghmans B, Nieman F, Lagro-Janssen T, Winkens R. Adherence to professional guidelines for patients with urinary incontinence by general practitioners: a cross-sectional study. J Eval Clin Pract. 2008;14(5):807-11.

6. Albers-Heitner P, Berghmans B, Nieman F, Lagro-Janssen T, Winkens R. How do patients with urinary incontinence perceive care given by their general practitioner? A cross-sectional study. Int J Clin Pract. 2008;62(3):508-15.

7. Teunissen TAM, Lagro-Janssen ALM. Sex differences in the use of absorbent (incontinence) pads in independently living elderly people: do men receive less care? Int J Clin Pract. 2009;63(6):869-73.

8. Albers-Heitner P, Berghmans B, Joore M, Lagro-Janssen T, Severens J, Nieman F, et al. The effects of involving a nurse practitioner in primary care for adult patients with urinary incontinence: The PromoCon study (Promoting Continence). BMC Health Services Research. 2008;8(1):84.

9. Albers-Heitner C, Lagro-Janssen A, Joore M, Berghmans L, Nieman F, Venema P, et al. Effectiveness of involving a nurse specialist for patients with urinary incontinence in primary care: results of a pragmatic multicentre randomised controlled trial. Int J Clin Pract. 2011;65(6):705-12.

10. Moore K, Wei Hu T, Subak L, Wagner T, Deutekom M. Committee 22. Economics of Urinary and Faecal Incontinence, and Prolapse. In: Abrams P, Cardozo L, Khoury S, Wein A, editors. Incontinence 4th International Consultation on Incontinence Paris July 5-8, 2008. Plymouth: Health Publication Ltd; 2009. p. 1708, 9.

11. Avery K, Donovan J, Peters T, Shaw C, Gotoh M, Abrams P. ICl-Q: a brief and robust measure for evaluating the symptoms and impact of urinary incontinence. Neurourol Urodynam. 2004;23(4):322-30.

12. The Euroqol Group. Euroqol - a new facility for the measurement of health-related quality of life. Health Policy. 1990;16(3):199-208.

13. Dolan P. A social tariff for EuroQol: results from a UK population survey. York: University of York 1995 Contract No.: 11.

14. Altman D. Practical statistics for medical research. Practical statistics for medical research. London: Chapman and Hall; 1991. p. 455-8.

15. Oostenbrink JB, Bouwmans CM, Koopmanschap MA, Rutten FFH. Handleiding voor kostenonderzoek [Manual for Cost Analysis]. Amstelveen: College voor Zorgverzekeringen. (In Dutch) 2004.

16. Health Insurance Board (CVZ). Guidelines for pharmacoeconomic research, updated version Valid from April 1st 2006. Diemen 2006.

17. Gold M, Siegel J, Russell L. Cost-effectiveness in health and medicine. New York: Oxford University Press; 1996. 
18. Koopmanschap MA, Meerding WJ, Evers S, Severens J, Burdorf A, Brouwer W. Handleiding voor het gebruik van PRODISQ versie 2.1 (PROductivity and DISease Questionnaire). Een modulaire vragenlijst over de relatie tussen ziekte en productiviteitskosten. Toepasbaar bij economische evaluaties van gezondheidszorgprogramma's voor patiënten en werknemers. Rotterdam/Maastricht: Instituut voor Medical Technology Assessment, Erasmus Universiteit Rotterdam/Instituut Maatschappelijke Gezondheidszorg, Erasmus Medisch Centrum/Beleid Economie en Organisatie van de Zorg, Universiteit Maastricht; 2004.

19. Manca A, Hawkins N, Sculpher M. Estimating mean QALYs in trial-based cost-effectiveness analysis: the importance of controlling for baseline utility. Health Econ. 2005;14(5):487-96.

20. Efron B, Tibshirani R. An introduction to the bootstrap. London: Chapman and Hall; 1993.

21. Buxton M. Economic Evaluation and Decision Making in the UK. Pharmacoeconomics. 2006;24(11): 133-1142.

22. Hout V, Al M, Gordon G, et al. Costs, effects and C/E ratios alongside a clinical trial. Health Econ. 1994:309-19.

23. Claxton K, Posnett J. An Economic Approach to Clinical Trial Design and Research Priority-Setting. Health Econ. 1996;5(6):513-24.

24. Statline database. 2010. Last update: 10-12-2009; numbers of 1-1-2009; [database on the Internet][cited 02-02-2010]. Available from: http://statline.cbs.nl/StatWeb/publication/.

25. Council for Public Health and Health Care (RVZ). Sensible and sustainable care [Zicht op zinnige en duurzame zorg]. The Hague 2006.

26. Chalkidou K, Lord J, Fischer A, Littlejohns P. Evidence-Based Decision Making: When Should We Wait For More Information? Health Aff 2008;27(6):1642-53.

27. Drummond M. Discussion: challenges in the economic evaluation of incontinence services. Urology. 1997;50(Supplement 6A):108.

28. Zwarenstein M, Treweek S, Gagnier JJ, Altman DG, Tunis S, Haynes B, et al. Improving the reporting of pragmatic trials: an extension of the CONSORT statement. BMJ. 2008;337(2):a2390.

29. Moher D, Hopewell S, Schulz KF, Montori V, Gotzsche PC, Devereaux PJ. CONSORT 2010 Explanation and Elaboration: updated guidelines for reporting parallel group randomised trials. BMJ. 2010;340:c869.

30. Schulz K, Altman D, Moher D. The Consort Group. CONSORT 2010 Statement: updated guidelines for reporting parallel group randomised trials. Trials. 2010;11(1):32.

31. Melville JL, Fan M-Y, Rau H, Nygaard IE, Katon WJ. Major depression and urinary incontinence in women: temporal associations in an epidemiologic sample. Am J Obstet \& Gynecol. 2009;201: 490.e1-7. 



\title{
Chapter 7
}

\section{Experiences and attitudes of nurse specialists in primary care regarding their role in care for patients with urinary incontinence}

\author{
Pytha Albers-Heitner \\ Toine Lagro-Janssen \\ Pieter Venema \\ Bary Berghmans \\ Ron Winkens \\ Ank de Jonge \\ Manuela Joore
}

Scandinavian Journal of Caring Sciences. 2011;25(2):303-310. 


\section{Abstract}

Aim To explore experiences and attitudes of nurse specialists in primary care regarding their role in care for patients with urinary incontinence (UI), thereby identifying facilitators and barriers for wider implementation.

Background Currently, primary care for patients with UI lacks sufficient adherence to existing guidelines on $\mathrm{UI}$ and is far from optimal. Studies in various countries show that involving nurse specialists may offer a solution to the inadequate care for UI. As qualitative studies on experiences of nurses with this type of intervention are lacking, we performed this study with a qualitative approach and data collection method within the course of a randomised controlled trial (RCT).

Method A focus group study was conducted in 2007 with six nurse specialists who were trained in caring for patients with $\mathrm{UI}$ in our pragmatic RCT. The focus group interview was audiotaped and transcribed verbatim. The data were analysed using qualitative content analysis to identify themes. To understand obstacles and incentives for change, we relied on an existing 'implementation model'.

Findings Nurse specialists feel competent to provide advice and information, to offer possible solutions and to give attention and guidance to the process of care of people with UI. They feel appreciated by patients and feel they offer an added value to the usual care of general practitioners (GPs). Nurses sometimes notice that GPs lack interest in UI. Personal contact with the GPs, availability of enough time, adequate equipment and financial resources are important preconditions for effective nurse specialist care. Nurse specialists value continuous education and feedback in daily care for patients with UI.

Conclusion Trained nurse specialists appeared to feel competent and satisfied to support GPs in care for patients with UI. They feel highly appreciated by both patients and GPs. 


\section{Introduction}

Shifting care from general practitioners (GPs) to nurse specialists may be a response to the increasing demand for healthcare because of ageing of the population. ${ }^{1,2}$ However, it can be difficult to change services and professional performance for the better. Much depends on the perspectives of users and the attitudes and behaviours of professionals in the context of their organisations and healthcare teams. ${ }^{3,4}$ In the Netherlands, we recently investigated the effectiveness and costeffectiveness of nurse specialist care for patients with urinary incontinence (UI) compared to care-as-usual by GPs. ${ }^{5}$ Considering the ageing of the population a further increase in prevalence of $\mathrm{UI}$ is expected. ${ }^{2,6}$ Both international and national evidence-based guideline recommendations on $\mathrm{UI}$ indicate that for most people with $\mathrm{UI}$ pelvic floor muscle and/or bladder training is the best non-invasive initial treatment to solve the problem, rather than just deal with the urine loss. ${ }^{7-13}$ At the moment, despite these guidelines, training is only incidentally offered by GPs, probably because it is too time-consuming. ${ }^{14-16}$ Most GPs choose an easier, but noncurative and ultimately far more expensive alternative: prescribing incontinence pads. More than $50 \%$ of people with UI use incontinence pads, especially older people. ${ }^{17}$ In recent years, there is a growing emphasis on redesign of professional roles and on shift of care in the healthcare sector and on employment of nurse specialists in primary care for chronic disorders. ${ }^{1,2}$ Shift of care is seen as a logical innovative development in offering healthcare, which contributes to a better quality of care, which is more patient friendly at the same time and efficiently organised. Nurse specialists have specific expertise and have more time to motivate patients than GPs. Studies in Canada, Australia, New Zealand, Scotland, USA, Norway and the United Kingdom show that involvement of nurse specialists may offer a solution to the inadequate care for UI with inappropriate prescription of incontinence pads. ${ }^{18-20}$ As cost-effectiveness is completely unknown, we performed a trial. After special training and using their specific expertise on $\mathrm{UI}$, we assumed nurse specialists would be competent in their new professional role to support the GP after the initial problem definition by the GP.

However, even when the involvement of the nurse specialist in her new role in $\mathrm{UI}$ care turns out to be effective, literature shows that actual change in daily practice is difficult to achieve. ${ }^{21}$ Because interaction of a range of factors at multiple levels may influence the success or failure of a new intervention by the nurse specialist in daily practice, an understanding of these factors (the obstacles and incentives for change) is crucial. ${ }^{21}$

To fully understand these factors, we relied on an 'implementation model' based on the one described by Grol et al. ${ }^{3,4,21}$ 


\section{The implementation model of Grol}

Based on a comprehensive overview of theories on implementation and behavioural change, Grol et al. ${ }^{3,4,21}$ developed an 'implementation model' for identifying potential barriers and promoters for change in clinical practice.

In addition, Grol et al developed a theoretical framework on the content and methods of process evaluation of an intervention which is extensively tested. ${ }^{22}$ The theories and the features in the model and the framework point to various potential barriers and facilitators for implementation. Factors include the competencies, attitudes, motivation for change and personal characteristics of the individual professionals who implement an intervention. They also relate to the characteristics or behaviour of patients, colleagues and others in the social network of the healthcare provider. Finally, they comprise the organisational, financial or structural aspects of the work environment (the system). Healthcare providers, practices and institutions can differ on any or all of these points. ${ }^{3,4,21}$ Therefore, when planning complex changes such as shift of care in practice ${ }^{1,2}$, in a process evaluation, 'potential barriers for quality of UI care' and 'factors facilitating change' at all relevant levels and related factors need to be addressed. One of these levels is 'the implementer', in this study the nurse specialist, who provides the intervention.

To our knowledge, no studies with a qualitative approach and data collection method are available on experiences and attitudes of nurses with this particular type of UI care shift from GPs to nurse specialists. Hence, further research is needed to explore facilitators and barriers for wider implementation of this shift of UI care in primary care. Therefore, we conducted a study with a qualitative approach and data collection method in addition to our study 'Promoting Continence' (PromoCon), a pragmatic multicentre randomised controlled trial (RCT) comparing nurse specialist care with care-as-usual for people with $\mathrm{UI}$ in general practice. ${ }^{5}$ Findings of the PromoCon trial will be reported elsewhere.

\section{Aim}

We conducted this study with a qualitative approach and data collection method in addition to a RCT to explore experiences and attitudes of nurse specialists in primary care regarding their role in care for patients with UI to identify facilitators and barriers for wider implementation. 


\section{Methods}

\section{Design}

We opted for a focus group study because we wished to use group dynamics to stimulate discussion and generate ideas. ${ }^{23,24}$ With this qualitative approach, we wished to find out what really matters to the nurses and detect experienced obstacles to changing performance. This would help explaining why improvement does or does not occur and the way quality of care for people with UI can be improved. ${ }^{23,24}$

\section{Participants}

Six nurse specialists from four Dutch regions took part in the focus group study. These nurse specialists provided care for patients with UI in the PromoCon study. The study was carried out from May 2005 until March 2008 in four Dutch regions (Maastricht, Nijmegen, Helmond, The Hague). All consecutive patients consulting their GP for UI within one year and patients already diagnosed with UI were eligible. People included in the RCT $(n=384)$ were followed during 12 months. After special training based on the implementation of evidence-based guideline recommendations on UI, the nurse specialist took over tasks from the GP related to specification of the diagnosis, treatment and monitoring of patients with UI. ${ }^{5}$ Four expert trainers on UI developed and provided the training programme, including diagnostic and therapeutic management of patients with the most prevalent types of UI. All nurses were successful in an individual competencies assessment. The first patient visit with the nurse specialist lasted one hour. Next, three to five weekly visits of 20 minutes were planned depending on patient's needs. Four follow-up visits of 15 minutes took place at six weeks, three, six and 12 months. Details of the training and intervention have been described elsewhere. ${ }^{5}$

\section{Data collection}

In October 2007, towards the end of the intervention period of the PromoCon trial, experiences and attitudes of all six nurse specialists were explored in one focus group study. Prior to the focus group study, we sent a short questionnaire to the participants to collect data on individual characteristics (age, education and nursing experience). Before and during the PromoCon study, all participating nurse specialists met on a regular base for feedback and case discussions. These meetings were prepared, coached and recorded (TL, CPAH) and chaired (TL) by UI specialists of the research team. Moreover, one researcher ( $\mathrm{CPAH})$ took notes during field observations (minimally two per nurse during the intervention period) that were discussed during the meetings. After each meeting during the trial period, the two 
researchers discussed the main issues that arose. Next, we developed a topic guide for the focus group study based on these issues and on the 'implementation model' as described in our introduction. ${ }^{3,4,21}$ The moderator started each topic with open questions. The first topic concerned the individual nurse specialists' experiences and attitudes towards the intervention. In addition, the nurse specialists discussed their experiences with patients with UI and GPs. Finally, the nurse specialists expressed their experiences with and attitude towards the practical organisation of their work. The focus group discussion took place at the university, where we organised earlier training and meetings of the nurse specialists. An independent moderator (AdJ) and an assistant (MS), both experienced in focus group interviews and confident with the topic of UI, conducted the focus group. To be able to explore nurses' genuine experiences and attitudes towards the training and the intervention, we found it essential to create a safe environment for an open discussion. To prevent socially desirable comments and to encourage less assertive participants, the moderator emphasised that we did not believe in right or wrong answers and that the participants would help us by expressing their opinions as openly as possible. During the focus group, the assistant took field notes and observed non-verbal communication. After the focus group, the moderator, the assistant and the main researcher (CPAH) discussed their impressions. We included these observations in the on-going analysis. The focus group lasted two hours and was recorded on audiotape.

\section{Ethical considerations}

The study was approved by the Medical Ethics Committees of all the involved medical centres and hospitals. The nurse specialists were informed about the aim of the focus group study, the voluntary participation and the anonymous and confidential handling of the research data. Subsequently, they agreed to participate in the focus group and gave their consent for the focus group to be tape recorded.

\section{Data analysis}

The questionnaire prior to the focus group was analysed using descriptive statistics. Data from the focus group study were analysed based on qualitative content analysis to identify themes. ${ }^{24,25}$

To increase dependability and confirmability, the interview was transcribed verbatim. ${ }^{23}$ To improve consistency and reliability of analyses, the external moderator (AdJ) and the researcher (CPAH) analysed the transcript independently of each other. ${ }^{25}$ First, codes were allocated to fragments of the transcript. The two researchers compared these and reached consensus about the set of codes to be used. 
The codes were grouped into categories, which were then developed into a more general analytical framework. To promote trustworthiness, we discussed all data fragments in the light of 'potential barriers and facilitators for quality of care and change' related to the individual's cognitive, educational, and motivational attributes, as well as to social, organisational and economic factors as described in the model of Grol. ${ }^{3,4,21}$

Next, the prominent themes were identified, and each category was allocated to the best fitting theme. To minimise bias, one other reader (TLJ) also studied the transcript and the three researchers discussed the themes that had emerged. The research group reviewed and commented on the final concept of the most important themes.

Four main themes were identified during the analysis and are discussed as follows: 'the competencies of the primary care nurse specialist in UI patients' care', 'the added value of the nurse specialist in care for UI patients in primary care', 'the cooperation between the nurse specialist and the GP' and 'preconditions identified by nurse specialists for wider implementation of this shift of care for UI in primary care'. Quotes (translated into English) are given to illustrate the findings. The following is the key to the quotes: [ ] = explanation by the authors, [...] = text left out.

\section{Findings}

\section{Characteristics of participants}

All six female nurse specialists filled in the questionnaire prior to the focus group study. They were Dutch diploma level nurses with four to 40 years of nursing experience (Table 1). Two nurses had a post registration continence qualification. Four nurses worked for home-care organisations, one nurse for a public hospital and one nurse both for a public hospital and for a home care organisation. The nurse specialists were invited by their own organisation to participate in the PromoCon study. They all believed that their organisations participated in the study to improve quality of care for $\mathrm{UI}$ and to be able to excel in this $\mathrm{UI}$ health counselling activity. The nurse specialists cared for a median number of 28 patients during the study. Per patient, the nurses gave seven consultations on average over the one-year intervention period. Half of the patients were visited at home. 
Table 1 Characteristics of nurse specialists $(N=6)$.

\begin{tabular}{ll}
\hline Age, median (min-max) & 40 (27-59) \\
Years of nursing experience, median (min-max) & $17(4-40)$ \\
Training hours prior to study for each nurse & 32.5 \\
Classroom education (14) & \\
Traineeship (12) & \\
Assessment (2.5) & 16 \\
Preparation (4) & 28 (9-69) \\
Hours group intervision during the trial & \\
Number of patients per nurse during study period, median (min-max) & 28
\end{tabular}

Using the 'implementation model' of Grol, our analysis highlights four main themes revealing factors that can either hamper or promote high quality of UI care and change. The themes found we called the 'competencies of nurse specialists', the 'added value of nurse specialists', the 'cooperation between nurse specialists and GPs' and finally the 'preconditions according to nurse specialists for wider implementation of this (new) UI care'. All factors found within the themes concern the individual's cognitive, educational and motivational attributes, as well as to social, organisational and economic factors as described in the model of Grol and the theoretical framework. ${ }^{3,4,21}$

\section{Competencies of nurse specialists}

Nurse specialists encountered people with UI frequently in their regular work, and they acknowledged UI as a serious health problem. For most nurses, previous experience with people with UI was restricted to the domiciliary care setting, meaning that care was mainly focused on providing incontinence pads. All nurses were very motivated for their new task in UI care. Their main reasons to participate were to enhance their professional role, provide more than only patient care, to develop a new specialty and to look for new challenges.

[...] wanted to specialise more in counselling various target groups yes and that that was a reason too [...]

Nurses really wanted to be competent to give useful information and education to people with UI and colleagues and, by doing that, be of support to GPs. Nurse specialists appreciated their own acquired competencies in guiding people with UI very much. They valued their education on UI with the possibility to call in experts and considered it an interesting enhancement of their professional role. 
[...] we started to observe very differently, you notice the difference with your colleagues who do not have that competence, who ask for your valuable contribution.

[...] I can teach people a lot, and because of that they are going to think for themselves and become active with their own body and [...] how can I, what can I do about it myself?

[...] she had too little result [...], a physiotherapist specialised in pelvic floor problems had to be consulted.

Nurse specialists experienced very good relationships with their patients. Nurses felt that most patients with $\mathrm{UI}$ were satisfied and happy with their care and therefore seemed to accept this care by nurse specialists. Nurse specialists could motivate people and experienced that patients' motivation was an important facilitating factor.

Nurses felt that even persons who only improved minimally were often satisfied because they understood their problem better. The fact that the nurse specialist paid attention to the UI problem and explained the relationship between $\mathrm{UI}$ and other physical or cognitive problems was mentioned regularly as an advantage.

[...] one lady for example suffered from recurrent cystitis and used almost always antibiotics; after visiting me for 3 months it was over.

[...] you start with how people sit on the toilet and then you look how they pee and whether they actually are able to relax their pelvic floor muscles, whether they have a good continuous flow [...] and you start with this during the first visit $[\ldots]$

\section{Added value of nurse specialists}

According to the nurse specialists, most people with UI did not realise that care from the nurse specialists was an alternative for care from their GP. Nurses had the impression that most patients with UI did not consult their GP for UI. If they had, GPs often prescribed pads and patients remained not aware of further treatment options. Consequently, the nurse specialist had an added value. Moreover, people with $\mathrm{UI}$ seemed to be satisfied with the information and advice they received and the attention that was given to their healthcare problem. People with UI often felt ashamed about their problem. Being able to visit people in their own homes where they felt more comfortable was seen as an advantage by the nurse specialists. Next to this, during the home visits, nurses had time and opportunity to record relevant 
contextual information and were better informed on the practical facilities in the neighbourhood.

[...] honestly I have to say, patients do not visit their GP that often, sometimes they mention something about incontinence to the GP, but very often patients only get pads, and [...] for all kind of reasons they do not actively take action on this problem.

[home visits are] very low threshold [...] you simply see incontinence very often in the district, because you always work with an older target group [...]

[...] I just said, and I want this to be recorded, I find that this often has to do with the fact that people have other problems too.

\section{Cooperation between nurse specialists and GPS}

During the intervention study, GPs worked with one nurse specialist. Overall, nurse specialists mentioned a good professional relationship with many GPs, while some paid special attention to build up a relationship with the GPs. When nurse specialists had personal contact with GPs and saw patients in the same area as the GP, the contact and the collaboration was excellent. Moreover, GPs initiated contact with nurse specialists easier. Nevertheless, many GPs were difficult to reach to discuss patients. This was even more difficult when nurse specialists had no personal contact with GPs before the start of the study. GPs were mostly contacted by the nurse specialist because she felt the need for more diagnostics on the pelvic floor function and to discuss the use of medication. Sometimes, too few diagnostic tests were performed by the GP and patients were inadequately informed.

[the nurse had asked the GP for additional physical examination of the pelvic floor muscle function] gives more information on [...], indeed that pelvic floor muscle is too tense after all, exactly what you missed since things did not improve.

Overall nurse specialists felt that GPs appreciated their competencies. Nonetheless nurses sometimes had the idea that GPs lacked interest in UI and GPs were not very willing to spend time for discussion.

[GPs said] I don't think UI is such a problem [...]

Now and then nurse specialists had the impression that GPs thought that nurse specialists were not competent or that GPs were in doubt whether UI was treated 
well. Finally, some nurses had the idea that GPs did not dare to hand over the care for UI patients to others.

Preconditions according to nurse specialists for wider implementation of this (new) Ul care

Training, supervision and tailored feedback of the nurse specialists were important facilitators for nurses specialists to feel competent to support GPs in adequate care for people with UI. Moreover, feeling competent enhanced their job satisfaction and stimulated their motivation to continue this care and to promote the possibilities of UI care to colleagues.

\section{[.... .that is what I learned [...] a wider view on the whole domain of UI [...]}

[...] many GPs are supported by a nurse for diabetes care, it would be excellent if every GP practice was supported by a continence nurse as well.

Personal contact between the nurse specialist and the GP clearly facilitates good professional contact and collaboration.

Nurses experienced that working conditions like efficiently organised time for patient care including home visits and patient report, adequate equipment and materials and enough financial resources are important preconditions for good quality of care.

\section{Discussion}

To our knowledge, this is the first study with a qualitative approach and data collection method that explored the experiences and attitudes of nurse specialists in primary care regarding their role in caring for patients with UI. Using the 'implementation model' and theoretical framework of Grol. ${ }^{3,4,21,22}$ we identified facilitators and barriers for wider implementation of this adaptation of the nurses' role and this reallocation of care from GPs to nurse specialists for patients with $\mathrm{UI}$ in primary care.

Moreover, this qualitative approach elicited disclosure of very personal feelings and experiences related to performance. ${ }^{23,24}$ As such, qualitative research nested in an experimental trial may clarify the improvements that adaptation of the nurses' role brings about in general practice.

The themes we found highlight important factors to consider that can either promote or hamper wider implementation of the intervention and are consistent with those mentioned in the 'implementation model' of Grol et al. Factors either 
involved the individual nurses, their social network, consisting of their patients in the study and the GPs they worked with, and the organisational system of their work.

We found that nurse specialists experience that their care offers an added value compared to the usual care of the GP for patients with UI: nurses have time and also possibilities to do home visits. Older patients often felt more comfortable at home and less ashamed to discuss UI. Next to this, nurses got relevant contextual information and could tailor their intervention to personal needs.

These experiences and the positive attitude of the nurse specialists are presumably related to the adequate training and feedback sessions the nurses received and the appreciation they experienced from patients and GPs. ${ }^{26}$ Moreover, we expect these positive findings to be influenced by the attention that has been given to a problem that was insufficiently addressed before by GPs. ${ }^{27}$

Our second finding is that nurse specialists feel they have a good collaboration with GPs. Cooperation improves when GPs work with one and the same nurse specialist and that there is personal contact with each other. Nurses experience that lack of interest of GPs in the problem UI, insufficient faith in the competence of nurses or the reluctance to shift of care can be important barriers to build up a good cooperation. These findings supported various theories and research findings that a positive relationship among healthcare providers is in general an important component of high quality patient care. ${ }^{28}$

The next finding concerns preconditions for nurse specialists for implementation of this shift of care for UI in primary care. According to the theory of planned behaviour, decisions are made according to personal models and beliefs about the changes about to be made and the perceived benefits and risks associated with them. ${ }^{29}$ Important prerequisites for the nurse specialists are that continuous training and feedback by experts is provided. In addition, it is important that personal communication on protocols, job description and process of care need to be finetuned with GPs. Efficient working conditions, such as agreement on time to discuss and report on patients, enough time for patient care including home visits, adequate equipment and materials and enough financial resources need to be taken care of. All these preconditions are known relevant issues to be considered not only by healthcare providers but also its planners and policy makers to successfully implement role revision that might improve inadequate care. ${ }^{30}$ Finally, we found that after special training, successful assessment and feedback sessions during the study period nurse specialists feel competent to care for patients with UI. This care is based on evidence-based UI guideline recommendations and strictly follows protocols while the GP continues to have the final responsibility. ${ }^{13,31}$ The nurse specialists are very satisfied with this enhancement of their professional role. They feel appreciated by patients and by GPs. 
These findings are consistent with findings in studies on disease management programmes in which care was protocolised. These studies showed that quality of care and satisfaction of patients was similar or increased when care was re-allocated to non-physicians compared to traditional care by doctors. ${ }^{32}$ Several studies on UI conclude that nurses are competent to care for people with $\mathrm{UI}$ and that this leads to improved health outcomes and patient satisfaction. ${ }^{18-20}$

This study had some limitations. We chose a qualitative focus group discussion to enable in-depth exploration of the relevant topics through the interaction between the nurse specialists. Usually, several interviews are needed until saturation of possible ideas is reached. As only six nurse specialists took part in the main study, only one focus group could be conducted. Nevertheless, we did not opt for individual interviews because we wanted to use group dynamics to stimulate discussion and generate best available ideas. ${ }^{24}$ Because the nurses knew we had an interest in the intervention they may have made socially desirable comments, although they commented on both positive and negative aspects of the intervention. Therefore, some bias may have occurred. Moreover, the limited number of nurses might limit the transferability and applicability of our results to similar situations in the primary care setting in a new context. Some GPs might prefer to allocate this task to their GP assistants or practice nurses in addition to other tasks. On the other hand, the six nurses seemed to represent a variety of nurses in home care settings, because they were working in areas with different urbanisation levels and they varied in age, years of working experience and educational background. ${ }^{33}$

Moreover, only the experiences of the nurse specialists are covered here. A more balanced picture would have emerged if a joint patient, GP and provider perspective had been offered combined with results of the trial. Future research should include interviews with patients and GPs and should be related to achieved costeffectiveness. However, despite the possible bias, we feel this qualitative study has provided a balanced overview of the important issues experienced by the nurse specialists to address for future implementation activities. ${ }^{3,4,21}$

Considering the ageing of the population and the expected increase of need for UI care nurses can therefore play an important role in UI care in the future. Implementation of this care in the existing healthcare structures seems feasible if experiences of the nurse specialists are taken into account to guarantee quality of care over time. ${ }^{2,4}$

\section{Conclusion}

In conclusion, trained nurse specialists appeared to feel competent and satisfied to support GPs in care for patients with UI. They feel highly appreciated by both patients and GPs. The intervention provided by the nurse specialists is likely to offer an 
added value to the usual care of the GP. Implementation of this adaptation of the nurse's role and shift of care from GPs to nurse specialists in the existing (health) care structures seem feasible if identified prerequisites identified by nurse specialists are met such as adequate training, supervision, feedback, financial resources and personal contact with GPs. These findings support an increased involvement of nurse specialists in Dutch primary care and contribute to the knowledge of the feasibility of this care provision by nurse specialists both from a international and from national perspective.

\section{Acknowledgments}

We are grateful to the nurse specialists who took part in this study and shared their experiences with us. Many thanks to Margriet Straver for assisting and typing the interview. 


\section{References}

1. Laurant M, Reeves D, Hermens R, Braspenning J, Grol R, Sibbald B. Substitution of doctors by nurses in primary care. Cochrane Database of Systematic Reviews 2004, (4): CD001271. DOI: 10.1002/14651858.CD001271.pub2.

2. Bodenheimer T, Chen E, Bennett D. Confronting The Growing Burden Of Chronic Disease: Can The U.S. Health Care Workforce Do The Job? Health Aff. 2009; 28: 64-74.

3. Grol R, Wensing M. What drives change? Barriers to and incentives for achieving evidence-based practice. Med J Aust. 2004; 180: S57-S60.

4. Grol R, Buchan H. Clinical guidelines: what can we do to increase their use? Med J Aust 2006; 185 301-2.

5. Albers-Heitner P, Berghmans B, Joore M, Lagro-Janssen T, Severens J, Nieman F, Winkens R. Study protocol. The effects of involving a nurse practitioner in primary care for adult patients with urinary incontinence: the PromoCon study (Promoting Continence). BMC Health Serv Res 2008: 8:84.

6. Melville JL, Katon W, Delaney K, Newton K. Urinary Incontinence in US Women: A Population-Based Study. Arch Intern Med. 2005; 165: 537-42.

7. Eustice $S$, Roe B, Paterson J. Prompted voiding for the management of urinary incontinence in adults. Cochrane Database of Systematic Reviews 2000, (2): CD002113. DOI: 10.1002/14651858.CD002113.

8. Ostaszkiewicz J, Chestney T, Roe B. Habit retraining for the management of urinary incontinence in adults. Cochrane Database of Systematic Reviews 2004, (2): CD002801. DOI: 10.1002/14651858.CD002801.pub2.

9. Ostaszkiewicz J, Johnston L, Roe B. Timed voiding for the management of urinary incontinence in adults. Cochrane Database of Systematic Reviews 2004,(1): CD002802. DOI: 10.1002/14651858.CD002802.pub2.

10. Wallace SA, Roe B, Williams K, Palmer M. Bladder training for urinary incontinence in adults. Cochrane Database of Systematic Reviews 2004, (1).: CD001308. DOI: 10.1002/14651858.CD001308.pub2.

11. Lagro-Janssen ALM, Teunissen D, Breedveldt-Boer HP, Dongen van JJAM, Lemain TJJ, Steeneken F, Dijkstra RH, Wiersma TJ. NHG-Standaard Incontinentie voor Urine M46 Eerste herziening (Dutch College Guideline on Urinary Incontinence. First revision). Huisarts Wet. 2006; 49 (2): 501-10.

12. Dumoulin $\mathrm{C}$, Hay-Smith J. Pelvic floor muscle training versus no treatment, or inactive control treatments, for urinary incontinence in women. Cochrane Database of Systematic Reviews 2010, (1): CD005654. DOI: 10.1002/14651858.CD005654.pub2.

13. Abrams $P$, Cardozo L, Khoury S, Wein A. Fourth International Consultation on Incontinence (ICI) - July $5-8,2008$. Palais des Congres, Paris, France. 2008, Paris, France Health Publication Ltd.

14. Kirby M. Managing stress urinary incontinence - a primary care issue. Int J Clin Pract. 2006; 60: 184-9.

15. Shaw C, Atwell C, Wood F, Brittain K, Williams K. A qualitative study of the assessment and treatment of incontinence in primary care. Fam Pract. 2007; 24: 461-7.

16. Albers-Heitner P, Berghmans B, Nieman F, Lagro-Janssen T, Winkens R. Adherence to professional guidelines for patients with urinary incontinence by general practitioners: a cross-sectional study. J Eval Clin Pract. 2008; 14: 807-11.

17. Albers-Heitner P, Berghmans B, Nieman F, Lagro-Janssen T, Winkens R. How do patients with urinary incontinence perceive care given by their general practitioner? A cross-sectional study. Int J Clin Pract. 2008; 62: 508-15.

18. Du Moulin MFMT, Hamers JPH, Paulus A, Berendsen C, Halfens R. The role of the nurse in community continence care: a systematic review. Int J Nurs Stud. 2005; 42: 479-92.

19. Williams KS, Assassa RP, Cooper NJ, Turner DA, Shaw C, Abrams KR, , Mayne C, Jagger C, Matthews R, Clarke M, McGrother CW. The Leicestershire MRC Incontinence Study Team. Clinical and costeffectiveness of a new nurse-led continence service: a randomised controlled trial. Br J Gen Pract. 2005; 55: 696-703. 
20. Williams KS, Assassa RP, Gillies CL, Abrams KR, Turner DA, Shaw C, Haslam J, Mayne C, McGrother $\mathrm{CW}$. A randomized controlled trial of the effectiveness of pelvic floor therapies for urodynamic stress and mixed incontinence. BJU Int. 2006; 98: 1043-50.

21. Grol RPTM, Bosch MC, Hulscher MEJL, Eccles MP, Wensing M. Planning and Studying Improvement in Patient Care: The Use of Theoretical Perspectives. Milbank Q. 2007; 85: 93-138.

22. Hulscher MEJL, Laurant MGH, Grol RPTM. Process evaluation on quality improvement interventions. Qual Saf Health Care. 2003; 12: 40-6.

23. Pope C. Qualitative methods in research on healthcare. Qual Saf Health Care 2002; 11: 148-52.

24. Bowling A. Unstructured interviewing and focus groups. Research methods in health. Investigating health and health services. 1997, Buckingham: Open University Press.

25. Silverman D. Methods. In: Interpreting Qualitative Data Methods for Analysing Talk, Text and Interaction (David S ed.), 1993 Sage Publications Ltd., London 30-90.

26. Hägglund D, Wadensten B. Fear of humiliation inhibits women's care-seeking behaviour for longterm urinary incontinence. Scand J Caring Sci. 2007; 21: 305-12.

27. Davis K, Kumar D. Pelvic Floor dysfunction: a conceptual framework for collaborative patient-centred care. J Adv Nurs. 2003; 43: 555-68.

28. Zwarenstein M, Goldman J, Reeves S. Interprofessional collaboration: effects of practice-based interventions on professional practice and healthcare outcomes. Cochrane Database of Syst Rev 2009, (3): CD000072. DOI: 10.1002/14651858.CD000072.pub2.

29. Ajzen I. The theory of planned behaviour. Organ Behav Hum Decis Process. 1991; 50: 179-211.

30. Laurant M, Harmsen M, Faber M, Wollersheim H, Sibbald B, Grol R. Revision of professional roles and quality improvement: a review of the evidence. Quest for Quality and Improved Performance (QQUIP). 2010; www.health.org.uk/QQUIP (last accessed 12 August 2010).

31. Laurant MGH, Hermens RPMG, Braspenning JCC, Sibbald B, Grol RPTM. Impact of nurse practitioners on workload of general practitioners: randomised controlled trial. BMJ. 2004; 328: 927-30.

32. Veer de AJE, Francke A, Plas M, Vogel B. Goede loopbaanmogelijkheden belangrijk voor verpleegkundigen en verzorgenden. Factsheet Panel Verpleegkundigen en Verzorgenden. 2008; 1-4. http://www.nivel.nl/panelvenv/(last accessed 12 August 2010). 


\section{Chapter 8}

Consumer satisfaction among patients and their general practitioners about involving nurse specialists in primary care for patients with urinary incontinence

Pytha Albers-Heitner

Ron Winkens

Bary Berghmans

Manuela Joore

Fred Nieman

Johan Severens

Toine Lagro-Janssen 


\begin{abstract}
Background Urinary incontinence (UI) is a very common problem, but existing guidelines on $\mathrm{UI}$ are not followed. To bring care in line with guidelines we planned an intervention to involve nurse specialists on $\mathrm{UI}$ in primary care and assessed this in a randomised controlled trial. Alongside this intervention we assessed consumer satisfaction among patients and general practitioners (GPs).

Methods Patients' satisfaction with the care provided by either nurse specialists (intervention group) or GPs (control group) respectively was measured with a self-completed questionnaire. GPs' views on the involvement of nurse specialists were measured in a structured telephone interview.

Results The patient satisfaction score on the care offered by nurse specialists was 8.4 (scale 1-10), versus 6.4 for care-as-usual by GPs. Over $85 \%$ of patients would recommend nurse specialist care to their best friends and $77 \%$ of the GPs considered the role of the nurse specialist to be beneficial, giving it a mean score of 7.2.

Conclusions Substituting UI care from GP to nurse specialist is welcomed by both patients and GPs. Small changes like giving additional UI-specific information and devoting more attention to UI (which had been given little attention before), would provide a simple instrument to stimulate patients to change their behaviour in the right direction.
\end{abstract}




\section{Introduction}

Urinary incontinence (UI) is a common problem with a prevalence of more than $5 \%$ among the population. ${ }^{1}$ Prevalence increases with age, particularly in women; prevalence is highest in elderly women. ${ }^{2} \mathrm{UI}$ is infamous for its impact on general wellbeing and social activities. ${ }^{3,4}$ When not treated, $\mathrm{UI}$ is a chronic, not self-limiting disorder with a strong tendency to worsen over time. It is one of the most important reasons for admittance of elderly people to nursing homes. ${ }^{4}$

Guidelines indicate that for most patients with UI in primary care, pelvic floor muscle and/or bladder training is the first-line non-invasive treatment to solve the problem rather than just compensate the urine loss. ${ }^{5-9}$ The problem so far with these guidelines is that they are followed only incidentally, irrespective of the type of guideline or clinical problem involved. ${ }^{10-13}$ Training for example is advised only incidentally by general practitioners (GPs), probably because it is time consuming. ${ }^{5,6,9,12,13}$ Most GPs prefer an easier, but non-curative and ultimately far more expensive alternative: prescribing incontinence pads. Thus, more than $50 \%$ of patients gets only incontinence pads, especially the elderly. ${ }^{1,13}$ In most cases, care (prescription of incontinence pads) is provided rather than cure (pelvic floor muscle and/or bladder training).

Costs for nursing home care and incontinence pads lead to a high financial burden on healthcare costs. Annually at least $€ 160$ million is spent on incontinence pads. Considering the ageing of the population a further increase in such expenditures is expected. ${ }^{5,6,14}$

By and large, UI can be divided into three main categories: stress or urgency UI and a mixed form. These are the most prevalent types in primary care, therefore this study mainly concerns these patients with stress, urgency, or mixed UI.

In several national reports the involvement of nurses in primary care to support GPs in improving care for patients with $\mathrm{UI}$ is recommended. ${ }^{5,6}$ First experiments with this kind of intervention seem to be effective and promising. ${ }^{15-17}$ Reasons for the effectiveness of a nurse specialist can be that nurses are capable of spending more time on motivating patients, apart from their specific expertise.

We studied the effects of involving nurse specialists on $\mathrm{UI}$ in a randomised trial with the objective to study whether involving nurse specialists as substitute and support for the GPs in managing UI leads to more or better effects on UI with the same or less costs or the same effects on UI with less costs. ${ }^{18}$ We found effects in terms of a reduced severity and impact of UI. ${ }^{18,19}$ In a addition to the trial, we were interested in perspectives of both UI patients and their GPs plus their experiences with the intervention of the nurse specialist. Therefore, in a survey we aimed at assessing the satisfaction of the 'consumers' being UI patients and their GPs plus their perceptions and experiences with the care provided by this new healthcare professional, the nurse specialist. 


\section{Methods}

Alongside a pragmatic randomised controlled trial ${ }^{18}$ we sent out a questionnaire in which we assessed satisfaction among patients on the involvement of nurse specialists for patients with UI. In the trial patients were randomly assigned to either an intervention group, receiving care by the nurse specialist or a control group, the latter receiving care-as-usual. After three and 12 months we assessed satisfaction and perceptions from patients both in the intervention group and the control group.

To measure patient satisfaction we sent out a questionnaire (available on request). Patients' satisfaction with care provided by the nurse specialist was originally planned to be measured with the, for UI, adjusted QUOTE self-completed questionnaire. $^{20}$ This questionnaire, however, was too unspecific for UI patients. Therefore, we have developed a new specific instrument. For the development of this questionnaire topics relevant to either the route via the nurse specialist or the usual care by the GP were identified in interviews with a panel of UI patients as 'hands-on' experts plus clinical experts on UI. We developed a set of items that enabled us to measure and test multi-items scales. Questionnaires were sent to UI patients who received care by the nurse specialist in the trial $(n=186)$ and patients who received care-as-usual in the trial $(n=198)$. Questions on overall satisfaction with the received care were included for all patients from both groups.

GP satisfaction plus their views on the availability and involvement of the nurse specialist were measured by a questionnaire (also available on request) by telephone after the study in a convenience sample of the participating GPs. From each participating region GPs working in a solo practice and who had at least one patient randomised to the nurse specialist were invited for the telephone questionnaire. From group practices one representative GP was asked for the questionnaire. Questions were asked on GPs opinion about the competencies of the nurse specialist (two), quality of care (two), effectiveness for the UI patient and the (added) value compared to usual care, meaningfulness/usefulness of this form of care (two) and their overall judgement/opinion (three). At the end of the interview GPs were explicitly asked for other comments which were recorded. The questionnaire was tested in a pilot study and modifications were made to get down to the definitive list. Answering categories were pre-coded. Data were collected by a trained independent research assistant.

\section{Data collection and analysis}

For the assessment of patient satisfaction a multivariate analysis of groups of preintended clusters of items was carried out by factor analysis (principal component analysis) using listwise deletion of missing items. Cronbach's alpha was used to 
measure internal consistency of the pre-intended scale(s) found as factors. Final scales were formed by summing the scores of the five-point Likert items belonging to each factor divided by the number of validly scored items. This way the resulting scale scores will always lie within the 1 (completely disagree) to 5 (completely agree) range. Minimally, half of the items belonging to the scale had to have valid (= non-missing) scores to obtain an overall scale score. Next, scores were transformed to a 1 (extremely bad) to a 10 (highly excellent) scale.

For the GPs questionnaire a more descriptive analysis was used. All data were analysed with SPSS-pc, version 12 and 15 (SPSS Inc, Chicago, IL, USA).

\section{Results}

\section{Satisfaction of patients with care received from the nurse specialist}

At three months patients in the intervention group ( $n=116 ; 62.4 \%$ ) gave a mean 8.45 (SD 1.02) on a scale from 0 (very bad) to 10 (excellent) to the nurse specialist and 8.34 (SD 1.16) at 12 months ( $n=125 ; 67.2 \%)$. When asking whether they would advise this way of care to their best friend, at three months $93 \%$ of the patients ( $n=$ 99; 53.2\%) (completely) agreed. At 12 months $(n=111) 86 \%$ still agreed.

In principal component analysis on items measuring satisfaction with care received from the nurse specialist we found eight factors (Table 1). Reliability of the eight scales was sufficient (Cronbach's range from $\alpha=0.61$ to $\alpha=0.86$ ). Overall scale mean for the evaluation of the eight scales after three months intervention of the nurse specialist ranges from 4.1 to 4.7 , so, the percentage of patients agreeing with these positively stated items is approximately $82 \%$ to $94 \%$. At the end of the study period the overall scale mean for the evaluation of the interventions ranges from 4.0 to 4.6. This means that the percentage of patients agreeing with these positively stated items is approximately the same at 12 months.

Regarding satisfaction of patients with care by the nurse specialist patients were also asked what care they received from the nurse specialist (Table 2). In principal component analysis on items measuring nurse specialist care three types were found. The first can be described as 'diagnostic actions', the second as 'information and advice received' and the third 'special treatment received'. Reliability of the three scales was however very low (Cronbach's $\alpha=0.36,0.46$ and 0.40 , respectively). 'Information on my problem and what to do' and on 'Referral to GP' were measured as single items. Almost all respondents reported after three months and 12 months that they got 'information on my problem and what to do' from the nurse specialist (97.4 and $91.7 \%$ respectively). Only few patients were referred back to the GP by the nurse specialist for further diagnostics, additional treatments or referral to other healthcare professionals. One third of the patients stated that 
extra diagnostic procedures were undertaken. Three quarter of patients got 'Special information and advice from the nurse specialist' (written explanation on bladder training (BT), pelvic floor muscle training (PFMT); advice on pad use). $85 \%$ Of patients reported on three and on 12 months respectively that they had received 'Special treatment by nurse specialist' (bladder diary, nurse gave advice on BT, PFMT and/or advice on home exercise BT, PFMT).

Table 1 Univariate descriptive statistics and reliability coefficients for eight scales belonging to satisfaction of patients with care received for UI from the nurse specialist 'Being given the opportunity to ask questions on UI', 'Explanation by the nurse specialist', 'The (in)adequacy of help to get insight in own healthcare situation', 'Advices on BT and PFMT', 'The level of difficulty of advice and information', 'The friendliness during treatment' and 'The professionalism of the nurse specialist', 'Making appointments'.

\begin{tabular}{|c|c|c|c|c|c|c|}
\hline \multirow{3}{*}{$\begin{array}{l}\text { Satisfaction with received care by the } \\
\text { nurse specialist }\end{array}$} & \multicolumn{4}{|c|}{ Response rate and scores } & \multirow{3}{*}{$\begin{array}{c}3 \\
\text { months } \\
\text { Cronba }\end{array}$} & \multirow{3}{*}{$\begin{array}{c}12 \\
\text { months } \\
\text { ach's } \alpha\end{array}$} \\
\hline & \multicolumn{2}{|c|}{3 months } & \multicolumn{2}{|c|}{12 months } & & \\
\hline & $\mathrm{n}(\%)$ & mean (SD) & $\mathrm{n}(\%)$ & mean (SD) & & \\
\hline Opportunity to ask questions on UI & $114(61.3)$ & $4.5(.66)$ & $120(64.5)$ & $4.4(.69)$ & $\alpha=0.86$ & $\alpha=0.77$ \\
\hline Explanation by the nurse specialist & $113(60.8)$ & $4.3(.69)$ & $125(67.2)$ & $4.3(.68)$ & $\alpha=0.61$ & $\alpha=0.65$ \\
\hline $\begin{array}{l}\text { (In)adequacy to give insight in health } \\
\text { situation }\end{array}$ & $117(62.9)$ & $4.2(.61)$ & $123(66.1)$ & $4.1(.70)$ & $\alpha=0.78$ & $\alpha=0.80$ \\
\hline Advice on BT and PFMT & $97(52.2)$ & $4.3(.75)$ & $102(54.8)$ & $4.4(.70)$ & $\alpha=0.68$ & $\alpha=0.73$ \\
\hline $\begin{array}{l}\text { Level of difficulty of advice and } \\
\text { information }\end{array}$ & $108(58.1)$ & $4.1(.81)$ & $115(61.8)$ & $4.0(.82)$ & $\alpha=0.63$ & $\alpha=0.52$ \\
\hline Friendliness during treatment & $118(63.4)$ & $4.6(.59)$ & $125(67.2)$ & $4.6(.63)$ & $\alpha=0.67$ & $\alpha=0.69$ \\
\hline Professionalism of the nurse specialist & $115(61.8)$ & $4.5(.58)$ & $126(67.7)$ & $4.4(.69)$ & $\alpha=0.77$ & $\alpha=0.77$ \\
\hline Making appointments & $107(57.2)$ & $4.7(.47)$ & $120(64.5)$ & $4.4(.69)$ & $\alpha=0.77$ & $\alpha=0.89$ \\
\hline
\end{tabular}

$(\mathrm{N}=186) 1$ = not satisfied at all 5 = very satisfied; BT, Bladder Training; PFMT, Pelvic Floor Muscle Training. 
Table 2 Univariate descriptive statistics on perceived care received from the nurse specialists as reported by respondents at 3 and 12 months. Scores range from 0 (never) to 100\% (always received).

\begin{tabular}{|c|c|c|c|c|}
\hline \multirow[t]{3}{*}{ Received type of care } & \multicolumn{2}{|c|}{3 months } & \multicolumn{2}{|c|}{12 months } \\
\hline & Freq & mean (SD) & Freq & mean (SD) \\
\hline & $186(100 \%)$ & & $186(100 \%)$ & \\
\hline $\begin{array}{l}\text { Diagnostic procedures } \\
\text { (urinary analysis, pad test) }\end{array}$ & $101(54.3)$ & $29.2(34.1)$ & $96(51.6)$ & $21.9(32.3)$ \\
\hline Information on problem and what to do & $114(61.3)$ & $97.4(16.1)$ & $120(64.5)$ & $91.7(27.8)$ \\
\hline $\begin{array}{l}\text { Special information and advice } \\
\text { (on BT, PFMT, adequate pad use) }\end{array}$ & $103(55.4)$ & $73.3(34.2)$ & $102(54.8)$ & $69.6(32.4)$ \\
\hline $\begin{array}{l}\text { Special treatment (bladder diary; advice on } \\
\text { BT, PFMT) }\end{array}$ & $117(62.9)$ & $85.3(23.1)$ & $122(65.6)$ & $85.1(25.1)$ \\
\hline Referral back to GP & $106(57.0)$ & $3.8(19.2)$ & $102(54.8)$ & $12.8(33.5)$ \\
\hline
\end{tabular}

BT, Bladder Training; PFMT, Pelvic Floor Muscle Training; GP, General Practitioner.

\section{Satisfaction of patients with care received from the GP}

At three months patients in the control group ( $n=66 ; 33.3 \%)$ gave a mean of 6.62 (SD 2.07) to the GP and after 12 months ( $n=68 ; 34.3 \%$ ) a mean of 6.74 (SD 2.20). Still, roughly $70 \%$ of these patients ( $n=39$ and $n=47$ respectively) answered they would advise care by the GP to their best friend stated, both after three and 12 months.

Patients were also asked what kind of care they received from the GP during the consultations when applicable (Table 3 ). In principal component analysis on items measuring care given by GP two factors were found. The first is defined as 'information from GP', the second 'additional testing by GP'. Reliability of the two intended scales was high (Cronbach's $\alpha=0.78$ and $\alpha=0.81$, respectively). Items on received 'prescription of medication', 'prescription of incontinence pads' and 'referral to the specialist' were measured as single items. Approximately half of the patients in the control group, who filled in the questionnaire, reported at three months intervention and at the end of the intervention period that they got a 'prescription for incontinence pads' from the GP (45.6 and $63.9 \%$ respectively). One third of the patients reported on three and on 12 months that they received 'information on making a bladder diary, explanation to do a pad test at home and/or on PFMT also at home'. Almost one out of five respondents stated that additional testing (urine analysis, physical examination, vaginal/rectal palpation, referral to a physiotherapist [PT]) was done by the GP. One out of four respondents had no formal contact with the GP on UI at all but presumably only with the practice assistant. 
Table 3 Univariate descriptive statistics on perceived care received from the GPs as reported by respondents at 3 and 12 months. Scores range from 0 (never) to $100 \%$ (always received).

\begin{tabular}{|c|c|c|c|c|}
\hline \multirow[t]{3}{*}{ Received type of care } & \multicolumn{2}{|c|}{3 months } & \multicolumn{2}{|c|}{12 months } \\
\hline & Freq & mean (SD) & Freq & mean (SD) \\
\hline & $198(100 \%)$ & & $198(100 \%)$ & \\
\hline $\begin{array}{l}\text { Additional testing (urine analysis, physical } \\
\text { examination, vaginal/rectal palpation, } \\
\text { referral to PT) }\end{array}$ & $73(36.9)$ & $14.7(30.3)$ & $73(36.9)$ & $18.7(29.8)$ \\
\hline $\begin{array}{l}\text { Information (bladder diary, explanation } \\
\text { pad test home, GP gave home PFMT) }\end{array}$ & $76(35.9)$ & $32.9(47.3)$ & $76(38.4)$ & $44.7(50.1)$ \\
\hline Prescription medication & $72(36.4)$ & $11.1(31.7)$ & 69 (34.9) & $17.4(38.2)$ \\
\hline Referral to specialist & $71(35.9)$ & $8.5(28.0)$ & $70(35.4)$ & $14.3(35.3)$ \\
\hline Prescription incontinence pads & 79 (39.9) & $45.6(50.1)$ & $83(41.9)$ & $63.9(48.3)$ \\
\hline
\end{tabular}

GP, General practitioner; PFMT, Pelvic Floor Muscle Training; PT, physiotherapist.

\section{Satisfaction of GPs with the nurse specialist}

In total, 65 GP practices participated in our intervention study. Of these 65, 49 where asked to be interviewed (the other $16 \mathrm{GPs}$ had no patients allocated to the intervention group). Of the 49 invited GPs, 40 (82\%) agreed to participate and were interviewed by telephone by an independent research assistant. GPs had a median of two (min one max 11) of their UI patients randomised to the nurse specialist. $32.5 \%$ Of the GPs had one patient treated by the nurse specialist, $40 \%$ two to four patients, $20 \%$ five to nine patients and $7.5 \%$ of the GPs more than 10 patients.

Three quarter of the GPs ( $n=32 ; 77 \%)$ stated that the support of a nurse specialist was a useful supplement to usual care for their UI patients, $10 \%$ did not (at all) think so and $10 \%$ had no opinion. The mean overall judgement of almost half of the GPs ( $n=19$ ) about the nurse specialist on a scale from 1 (absolutely insufficient) to 10 (excellent) was 7.2 (SD 0.84 ), the rest had no opinion. 50\% Of the GPs would recommend the support by the nurse specialist to colleagues, one third would not do so and $13 \%(n=5)$ had no opinion (yet).

23 GPs made remarks. The most important positive remarks being: 'Because of the project we now pay more attention to UI in our practice'; 'At the moment there are too many different professionals, we would like to give this care to our UI patients together with professionals in our usual network'; 'It could be a task for the practice nurse in our practice'; 'Accessibility is important'; 'Meaningful because of ageing population'.

A few negative remarks were: ' $\mathrm{UI}$ is not a priority for the GP'; 'We cooperate with the physiotherapist on UI for many years, so we already covered a good care for UI patients'. 


\section{Discussion}

The involvement of nurse specialists was highly appreciated among patients and GPs were by and large also positive. It showed an added value for patients to tackle their UI problem, to supply advice and information, to offer possible solutions, to support motivation of $\mathrm{UI}$ patients and to relieve the GP of tasks. We expect this to be caused mainly by the attention that now has been given to a problem insufficiently addressed before by GPs.

\section{Strengths and weaknesses of the study}

Important elements were that the study included both patients receiving nurse specialist care and patients receiving GP care over a one-year period. The study adds new data to existing knowledge, as similar data on the long-term perspective of patients and GPs on nurse care in primary care are scarce. ${ }^{21}$

The collection of data for the most important outcome parameters was mainly dependent on the co-operation of participating patients and their willingness to provide data through questionnaires and diaries. Compared to the collection of observational data this bears a risk of non-response. This risk was particularly present as the questionnaires were sometimes very extensive due to the design of the trial. Repeated motivation of patients was therefore needed. However, in the control group only one out of three responded, as we assume mainly because of not receiving specific care-as-usual in the control group. 95\% of patients in the study had UI for a longer period. This may have played a positive role in the motivation to follow the intervention and fill in the questionnaires. Still, we were repeatedly confronted with missing values or incomplete questionnaires in the control group.

\section{Patient satisfaction}

The great majority of the participants was very satisfied with the care they received by the nurse specialist and would recommend this care to their best friend in case of UI. Presumably, the extra and intense attention of the nurse specialist for the UI problem (where it usually has not been addressed before by the GP) together with the indeed reduced impact and severity of urine loss is the main factor responsible for this positive view. These results are in line with other studies with substitution of care by nurses in primary care. ${ }^{21}$ The satisfaction with care provided by the nurse is higher than satisfaction with care by the GP. This difference is however not surprising considering the extra Ul-specific information and attention that patients get from the nurse specialist. Patients, however, in our view do not seem to be dissatisfied with UI care by the GP although the scores lie within the lowest score range found in nationwide Dutch studies on general patient satisfaction. ${ }^{22}$ 


\section{GP satisfaction}

The 40 participating GPs in the interview had an average of only two patients randomised to the nurse specialist. This may explain why some GPs would not give their opinion on the intervention. Nonetheless about half of these GPs were positive about the use of the route through the nurse specialist in a sense that it was a useful supplement to the usual care for their UI patients. GPs gave an overall judgement of 7 on a scale of 0 to 10 (excellent) to the nurse specialist care, and recommended this support by the nurse specialist to their colleagues.

The extra positive and negative remarks GPs made at the end of the interview reveal some interesting facilitators and barriers for future implementation of this intervention for UI patients. Giving attention to the problem of UI ('Because of the project we now pay more attention to $U I$ in our practice') and an active attitude towards UI is recommended through all UI guidelines to improve quality of care. ${ }^{5,8,22}$ Moreover quality of UI care might be improved when this care could be implemented in the existing primary care networks ( '...we would like to give this care to our UI patients together with professionals in our usual network' and 'It could be a task for the practice nurse in our practice' ). This improvement can be made with easy accessibility and involvement in the monitoring of the ageing population with several complex co-morbidities.

Altogether, given the positive response by patients and GPs as consumers of substituted care by nurse specialists and taking into account the positive experiences of the nurse specialists during the intervention, we can conclude that substituting $\mathrm{UI}$ care from GP to nurse specialist is welcomed by both patients and GPs. However, as $\mathrm{UI}$ is a chronic disorder with a natural tendency to increase over time, it may be difficult to keep patients motivated on the long run. This would argue in favour of a prolonged and repeated intervention by a trained healthcare professional. 


\section{References}

1. Health Insurance Board (CVZ). GIP-peilingen. Total cost 2002-2006. 2007.

2. Teunissen TAM, Bosch van den WJHM, Hoogen van den HJM, Lagro-Janssen ALM. Prevalence of urinary, fecal and double incontinence in the elderly living at home. Int Urogynecol J 2004;15(1):10-3.

3. Norton P, Brubaker L. Urinary incontinence in women. Lancet. 2006;367(9504):57-67.

4. Abrams P, Cardozo L, Khoury S, Wein A. Fourth International Consultation on Incontinence (ICI) - July 5 - 8, 2008. Palais des Congres, Paris, France. Paris, France Health Publication Ltd; 2009.

5. Health Council of the Netherlands (Gezondheidsraad). Urine Incontinentie [Urinary Incontinence]. Publication no. 2001/12. The Hague 2001. Report No.: ISBN: 90-5549-375-9.

6. Health Insurance Board (CVZ). Urine incontinentie bij volwassenen [Urinary Incontinence in adults]. Diemen 2003 Contract No.: 2003/147.

7. Teunissen T, Jonge de A, Weel van C, Lagro-Janssen ALM. Treating urinary incontinence in the elderly-conservative measures that work: A systematic review. Fam Pract. 2004;53(1):25-30, 2.

8. Lagro-Janssen ALM, Teunissen D, Breedveldt-Boer HP, Dongen van J, Lemain TJJ, Steeneken F, et al. NHG-Standaard Incontinentie voor Urine M46 Eerste herziening [Dutch College Guideline on Urinary Incontinence. First revision]. Huisarts Wet. 2006;49(2):501-10.

9. Newman D, Ee C, Gordon D, Srini V, Williams K, Cahill B, et al. Committee 21. Continence Promotion, Education and Primary Prevention. In: Abrams P, Cardozo L, Khoury S, Wein A, editors. Incontinence $4^{\text {th }}$ International Consultation on Incontinence Paris July 5-8, 2008. Plymouth: Health Publication Ltd; 2009.

10. Kirby M. Managing stress urinary incontinence - a primary care issue. Int J Clin Pract. 2006;60(2):184-9.

11. Grol R, Buchan H. Clinical guidelines: what can we do to increase their use? Med J Aust. 2006;185 (6):301-2.

12. Albers-Heitner P, Berghmans B, Nieman F, Lagro-Janssen T, Winkens R. Adherence to professional guidelines for patients with urinary incontinence by general practitioners: a cross-sectional study. J Eval Clin Pract. 2008;14(5):807-11.

13. Albers-Heitner P, Berghmans B, Nieman F, Lagro-Janssen T, Winkens R. How do patients with urinary incontinence perceive care given by their general practitioner? A cross-sectional study. Int J Clin Pract. 2008;62(3):508-15.

14. Melville JL, Katon W, Delaney K, Newton K. Urinary Incontinence in US Women: A Population-Based Study. Arch Intern Med. 2005;165(5):537-42.

15. Du Moulin MFMT, Hamers JPH, Paulus A, Berendsen C, Halfens R. The role of the nurse in community continence care: a systematic review. Int J Nurs Stud. 2005 5;42(4):479-92.

16. Williams KS, Assassa RP, Cooper NJ, Turner DA, Shaw C, Abrams KR, et al. Clinical and costeffectiveness of a new nurse-led continence service: a randomised controlled trial. $\mathrm{Br} J$ Gen Pract. 2005;55(518):696-703.

17. Williams KS, Assassa RP, Gillies CL, Abrams KR, Turner DA, Shaw C, et al. A randomized controlled trial of the effectiveness of pelvic floor therapies for urodynamic stress and mixed incontinence. BJU Int. 2006;98(5):1043-50.

18. Albers-Heitner P, Berghmans B, Joore M, Lagro-Janssen T, Severens J, Nieman F, et al. Study protocol. The effects of involving a nurse practitioner in primary care for adult patients with urinary incontinence: the PromoCon study (Promoting Continence). BMC Health Serv Res. 2008(8):84

19. Albers-Heitner C, Lagro-Janssen A, Joore M, Berghmans L, Nieman F, Venema $P$, et al. Effectiveness of involving a nurse specialist for patients with urinary incontinence in primary care: results of a pragmatic multicentre randomised controlled trial. Int J Clin Pract. 2011;65(6):705-12.

20. Sixma H, Campen C van, Kerssens J, L. P. De QUOTE-vragenlijsten. Kwaliteit van zorg vanuit patientenperspectief; vier nieuwe meetinstrumenten. Onderzoeksprogramma kwaliteit van zorg. Utrecht: NIVEL/ NWO /VWS1998. 
21. Laurant M, Harmsen M, Faber M, Wollersheim H, Sibbald B, Grol R. Revision of professional roles and quality improvement: a review of the evidence. London: The Health Foundation. 2010 Contract No.: 6_suppl.

22. Abrams P, Andersson KE, Birder L, Brubaker L, Cardozo L, Chapple C, et al. Fourth international consultation on incontinence recommendations of the international scientific committee: Evaluation and treatment of urinary incontinence, pelvic organ prolapse, and fecal incontinence. Neurourol Urodynam. 2010;29(1):213-40. 
Chapter 9

General Discussion 


\section{General discussion}

Doubts about the quality, efficiency and organisation of care for urinary incontinence (UI) and the rising socio-economic burden imposed by UI on the healthcare system, have made it increasingly important to find solutions to improve all aspects of UI care. ${ }^{1,2}$ The main objective of the studies of the research project reported on in this thesis was to study whether the availability and involvement of trained nurse specialists in primary UI care is feasible and leads to positive effects on patient outcomes and to cost-effective care for adult UI patients, compared to care-as-usual.

This chapter discusses general aspects of the studies and their findings. A summary of the main findings reported in the chapters is presented, from which an overall project conclusion is derived about the role of nurse specialists in primary care for UI patients. The chapter also addresses methodological considerations about the studies, and compares our results with the literature and recent developments in the prevention of incontinence and the organisation of continence care. This is followed by a discussion of the overall implications and recommendations for further research and healthcare practice, and the chapter ends with our overall conclusion regarding the involvement of trained nurse specialists in primary care for Ul patients.

\section{Main findings}

Part I of this thesis discusses barriers to efficient primary UI care, based on two cross-sectional surveys (one among GPs and one among UI patients). GPs were found to only partly adhere to their guideline on UI care in terms of diagnosis and treatment, and one out of four GPs agreed that it was difficult to adhere to this guideline, mainly due to lack of time, staff, diagnostic tools, competencies to provide this care and low motivation of patients. UI patients using pads reported suboptimal and unsatisfactory GP care and management. One out of three reported that they had had no direct contact with their GP for their first pad prescription, and half of the UI patients who visited their GP only had pads prescribed.

Part II of this thesis reports on the pragmatic multicentre PromoCon randomised controlled trial (RCT), comparing a one-year intervention by trained home-care and hospital-linked nurse specialists with care-as-usual, after initial diagnosis and assessment by GPs of adults with stress, urgency or mixed UI.

Although no differences between the groups were found in terms of the oneyear linear trend, patients within both study groups improved significantly in terms of UI severity and impact on UI-specific health-related quality of life (HRQOL). After correction for effect modifiers (UI type, body mass index) we found significant differences between groups in favour of the intervention group at three months. After 
one year, patients in the intervention group who had no anxiety/depression at baseline had improved significantly more than those in the care-as-usual group.

Compared to care-as-usual, involving nurse specialists in primary care for UI patients had a cost-effectiveness ratio of $€ 16,742$ per Quality Adjusted Life Year from the societal perspective ( $\left.Q A L Y_{\text {societal }}\right)$ gained. Decision uncertainty was considerable:

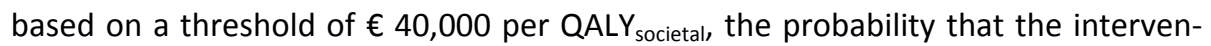
tion is cost-effective was $58 \%$. The 5 -year value of perfect information in the Netherlands amounted to $€ 78$ million, indicating that further research into the management of UI in primary care is worthwhile.

Involvement of nurse specialists in UI care was considered a positive intervention by both the nurse specialists and the consumers, that is, the UI patients and their GPs. The nurse specialists felt competent, appreciated and satisfied about supporting GPs in the care for UI patients. Contacts with the GPs, education and personal feedback, time, equipment and financial resources were important prerequisites for effective nurse specialist care.

Over $85 \%$ of patients would recommend nurse specialist care to their best friends and $77 \%$ of the GPs considered the role of the nurse specialist to be beneficial.

Based on these results, we concluded that the availability and involvement of trained nurse specialists in primary $\mathrm{UI}$ care is feasible and leads to favourable effects in terms of patient outcomes and to cost-effective care in adult UI patients compared to care-as-usual. Given these results, together with the considerable decision uncertainty surrounding them, we recommend adopting the intervention in routine practice, while at the same time conducting outcomes research to further reduce uncertainty. $^{3}$

\section{Methodological considerations}

\section{Health technology assessment}

The overall approach of our research project was that of the Technology Assessment Iterative Loop (TAIL). ${ }^{4}$ The different parts of this project reflect the consecutive steps of TAIL: causes of burden of illness (step 2), effectiveness (step 3), efficiency (step 4) and feasibility as part of the fifth step of synthesis and implementation. A strong aspect of our research project was therefore that we used different quantitative and qualitative research methods to evaluate the various aspects of our intervention. ${ }^{5}$ This combination of qualitative studies along with RCTs to explore processes, contextual factors and intervention characteristics that may have influenced trial outcomes remains uncommon. ${ }^{6}$ 


\section{Causes of the burden of $\mathrm{UI}$}

A limitation of our studies to assess the causes of the burden of UI (chapters 2 and 3 ) is that they relied on self-reported retrospective data, at both GP and patient level. As regards GPs, it is likely, and in fact known, that the non-responders might have less knowledge about and interest in $\mathrm{UI},{ }^{7,8}$ so the GP results may have been biased in a positive direction. Furthermore, using the patients - by and large a specific patient population of pad users- as a source in assessing the causes of the burden may have led to biased information. The patients' perception of the condition and their ideas about the efficacy of available interventions might influence their decision to contact a care provider and the way they express their request for care, and hence also the actual care provided by the professional. ${ }^{9,10}$ Therefore, our efforts to identify and assess possible causes of the burden of UI might have benefited from the use of medical records in addition to the survey methods we used. ${ }^{11}$

\section{Effectiveness}

Effectiveness of nurse specialist involvement was evaluated in our multicentre pragmatic RCT, which might reflect a biased reality. In addition to the specific reasons mentioned in previous chapters, there are three general reasons why our pragmatic design may not reflect the UI care by nurse specialists under routine practice conditions. ${ }^{12,13}$

First, the trial used an active recruitment strategy to include patients within a limited time frame. Less than one out of five UI patients, mostly female, of the white race and previously diagnosed, finally participated in the trial. Because of this overrepresentation of white women, which is to some extent inherent in the prevalence of the disorder, our results do not allow any conclusions about gender or race and ethnicity differences which play a role in help-seeking behaviour, communication on available treatments and patients needs. ${ }^{14,15}$ Most of the included patients had a fairly long history of UI (with mean UI duration of 10 years, SD 11), and incident cases may have been underrepresented. A lack of efficacy of the intervention can be expected in patients with a longer duration of the condition and more severe complaints. ${ }^{16,17}$ In addition, the active recruitment strategy may have made patients in the control arm aware of the severity of their condition and the treatments available. In this respect, the experiences of the research assistants who made the phone calls for final inclusion may be illustrative. They found that the telephone calls were considerably longer than is usual for calls to include patients in trials on other health conditions. Many UI patients told them that this was the first time that any attention had been paid to their UI problem, that a treatment had been offered, and that they had had the opportunity to say how much they were bothered by their UI. This awareness may have jeopardised the contrast between experimental and control arms. 
Secondly, some consequences of UI treatment may take considerable time to become manifest. The 12-months follow-up may have been too short to capture all benefits, such as changes to patients' lifestyle, use of pads and the healthcare services provided (e.g. fewer surgical procedures). ${ }^{18-21}$

Finally, only six nurse specialists, differing in terms of age, years of working experience and educational background, and working in a variety of home-care and hospital-linked settings, provided the intervention for 186 UI patients from 65 GP practices in areas with different levels of urbanisation. In addition, the nurses were highly motivated and well facilitated during the project. $^{22}$

Given the wide variety and the specific characteristics of Dutch medical practices, the transferability and applicability of our results to similar situations in primary care settings in other contexts (for instance also in other countries) remains to be established. $^{23}$

In summary, although we consider our trial to be pragmatic based on the criteria of the CONSORT extension for pragmatic trials that builds upon the main CONSORT statement criteria, ${ }^{24,25}$ a more ideal trial should have used naturalistic patient recruitment, a longer follow-up, and a larger and more representative sample of UI care providers.

\section{Efficiency}

Since we performed an economic evaluation from the societal perspective alongside a pragmatic RCT in routine healthcare, our data can be used to guide coverage decisions. It has been proposed in the Netherlands that the appraisal of costeffectiveness in terms of costs per QALY should depend on the burden of illness. ${ }^{26}$ The Dutch Council for Public Health and Health Care (RVZ), does not define a fixed threshold for costs per QALY, but uses values ranging from $€ 10,000$ for a limited burden of illness to $€ 80,000$ for the most severe burden of illness. So far, however, little is known about society's willingness to pay (WTP) for an additional QALY when conservative UI management is involved. ${ }^{21}$

Another problem is that generic HRQOL as an outcome measure from the patient's perspective may lack responsiveness in UI. We therefore developed the Incontinence Severity weighted Life Year (ISLY, a linearly transformed sum score based on the International Consultation on Incontinence Questionnaire Short Form [ICIQ-UI SF]) as a sensitive and disease specific outcome measure additional to the QALY. ${ }^{27}$ However, the interpretation of the costs per ISLY gained is currently still difficult. In this respect, it would be valuable to have an indication of the societal threshold of the costs of a UI-free life year as measured in ISLYs.

Overall, we consider our economic evaluation to be a sound evaluation from the societal perspective, based on criteria for performing and reporting economic evaluations. ${ }^{28,29}$ Nevertheless, a more ideal trial, as described above, should have 
allowed the calculation of cost-effectiveness over a longer time horizon, which could have reduced uncertainty. In addition, if we had found significant clinically relevant differences between the experimental group and the care-as-usual group, in terms of decreasing the impact of UI on daily life, and thus the use of incontinence pads, over a longer time horizon, we could have used a Markov health state transition model to calculate the long-term cost-effectiveness of the intervention.

\section{Feasibility}

We were only able to perform one qualitative focus group study, given the limited number of six nurse specialists (chapter 7). The study was based on criteria of the 'implementation model' and theoretical framework developed by Grol et al. ${ }^{30-33}$ These nurses were highly involved, supported and motivated. Although patients in the intervention group were more satisfied about the care they had received than those who had received care-as-usual, this comparison should be interpreted with caution in view of the low response rates. We assume that this was mainly because of the questionnaires were sometimes very extensive due to the design of the trial and because of not receiving specific care-as-usual in the control group.

Half of the GPs we interviewed were positive about the involvement of the nurse specialist in the treatment of their UI patients. Two aspects have to be carefully considered when interpreting this finding. First, of the 65 GP practices we contacted, only 49 met the inclusion criterion for the interview of having at least one patient randomised to the nurse specialist. This criterion is obviously too little to have an opinion about this change in the organisation of care. Despite all these limitations, we feel that involving trained nurse specialists in UI primary care management seems feasible when certain prerequisites for the nurses have been met and issues reported by patients and GPs are carefully addressed. However, given the limitations, the generalisability of our findings must be interpreted with caution. Wider implementation and outcomes research should be undertaken to reproduce the present studies of feasibility as a quality indicator, from the viewpoint of both the patients and the health professionals involved.

\section{Comparison of our results with the literature and recent developments in UI care}

Although cost-effectiveness evidence is becoming more important in healthcare decision making, this type of evidence has so far been scarce in the field of initial conservative treatment of $\mathrm{UI}$ in primary care. In contrast, there is increasing evidence of the effectiveness of this conservative treatment, involving lifestyle advice, bladder training and pelvic floor muscle training, for the most prevalent types of 
UI. ${ }^{34,35}$ In addition, more and more economic evaluations from the societal perspective have become available for UI medications and more specialised UI treatments like surgery. Surprisingly, the study presented in this thesis is, to our knowledge, still the first full economic evaluation alongside a pragmatic RCT both from the societal and the patient's perspective regarding initial primary care UI management. ${ }^{20,21,36-38}$ The few available cost-effectiveness analyses regarding initial conservative UI management are not comparable with our results, because of differences in the methodology used, the target population, the qualifications of the care providers and programme aspects. Different local circumstances make international transferability of cost-effectiveness results difficult or even impossible. ${ }^{39}$ Nevertheless, fin-dings of studies of healthcare costs associated with general nurse role revision are comparable to our findings: depending on the context of care and the specific nature of role revision, effects are mixed, with no cost savings (no increase in costs, 6 out of 9 reviews reporting reduction in costs, no cost savings). ${ }^{20}$

In addition, there have been positive developments in the prevention of incontinence and the organisation of continence care since this project started in 2005 stimulating effective and efficient care. ${ }^{1,40,41}$ Various efforts are being made to increase the uptake of UI guidelines by the public, UI patients and healthcare professionals, by creating awareness, accessibility and service capacity. ${ }^{10,42-47}$ Moreover, multidisciplinary UI guidelines have become available, facilitating collaboration both in primary care between GPs and physiotherapists ('Landelijke Eerstelijns Samenwerkings Afspraak', (LESA), a UI agreement between GPs and pelvic physiotherapists ${ }^{48}$ and between all relevant healthcare professionals for the elderly, ${ }^{49}$ while some other ones are under construction. ${ }^{50}$ However, despite these efforts to implement evidence-based care for UI, there is still little evidence that these activities lead to to actual behavioural change. ${ }^{10,51}$

In short, although comparability with other studies and developments in UI care is limited, our findings confirm that suitably trained nurse specialists are capable of undertaking tasks in UI care that were previously performed only by doctors, that patients are equally or even more satisfied with the care provided by these nurses compared to doctors, and that the effectiveness and efficiency of UI care can be improved. $^{52-55}$

In recent years, there has been an increasing shift from specialist care to primary care, and from doctors to nurse specialists, especially for chronic disorders. ${ }^{1,20,40,56}$ In primary care, there is growing emphasis on involving nurse practitioners [called Praktijk Ondersteuners, or POHs, in Dutch] for various health problems in general practice, but this has not yet been widely implemented for UI. However, as a follow-up to the studies presented in this thesis, and in view of the limited availability of nurse specialists, two pilot implementation studies have been started which might serve as examples of how to tackle potential barriers and how to facilitate wider implementation. ${ }^{51}$ One pilot study is in progress in the Nijmegen region, 
training POHs to play a major role in UI management. ${ }^{57,58}$ The feasibility of the intervention by well-trained POHs in general practice is very high: they are closely associated with the GP practice and the interventions fit with their other general practice tasks such as diabetes and COPD care, which are related to UI. Preliminary interviews and feedback forms have shown that the $\mathrm{POH}$ perceive the training as expanding their competencies, which increases their enthusiasm and motivation. Up to now, the $\mathrm{POH}$ have included around five patients per practice in six months. Three out of four UI patients reported an improvement or complete remission of their UI, as assessed by micturition diaries and the Sandvik scale (comparable to the ICIQ-UI SF in diagnosing the type of UI). ${ }^{17}$

As a part of the IMplementation of PromoCon (IMPromoCon) programme, a further pilot project is in progress in the Maastricht region. ${ }^{59}$ Its objective, also based on the results of the PromoCon trial and the LESA agreement ${ }^{48}$, is to optimise $\mathrm{UI}$ care in primary and secondary care settings, by implementating the so-called Quattro model. ${ }^{60}$ This model provides systematic organisation and cooperation between primary care, i.e. GPs, nurse specialists or POHs and pelvic physiotherapists, and secondary care with its pelvic care nurse specialists, pelvic physiotherapists and medical specialists.

\section{Overall research recommendations}

The studies presented in this thesis have provided research findings that are useful as a basis for further studies. First of all, the PromoCon trial involved patients being treated for one year, in an intensive scheme, with five to seven visits during the first three months, followed by two consultations at six and 12 months to monitor effect and adherence. An additional contact, between six and 12 months, to improve adherence and increase the stability of the effects, might be considered given the decline of the effect of the intervention between three and 12 months, while a longer follow-up by the nurse specialists might also be considered.

As indicated in our overall conclusion, we recommend initiating outcomes research in the field of primary care for UI. ${ }^{3}$

Such observational studies should be based on the following starting points. Firstly, the prerequisites identified by nurse specialists, patients and GPs should be met, such as sufficient training for nurses, personal contacts between nurse specialists and GPs to share experiences, and provide supervision and feedback, and sufficient financial resources. Secondly, GPs and nurse specialists should inform UI patients and their care givers better about the appropriate quality of care, in the hope that they will ask for such treatments in future consultations. ${ }^{51}$ Thirdly, UI care should fit in with various existing situations and circumstances, while using the design and findings of our research project, which will require some artistry in organis- 
ing the intervention. ${ }^{61}$ Fourthly, the sample should be based on unselective patient recruitment from daily practice, using a larger sample of UI care providers to allow for qualitative research with satisfactory methodological quality. In addition to the survey methods used in our studies, medical records should be used to assess the causes of the burden of UI. Only then can a more complete and balanced picture be obtained by integrating qualitative data from all parties involved at the relevant levels with quantitative data. ${ }^{62}$ Careful monitoring of the performance of the intervention with effective patient and provider registries of real world data should enhance awareness in all those involved, allow for longer follow-up with future evidence development and yield further information for coverage decisions. ${ }^{11,63}$

To this end, we recommend using the ICIQ-UI SF severity sum score (frequency, amount and impact of UI on HRQOL) as an indicator of the effectiveness of various treatments offered in routine practice in terms of UI health status. At the same time, the ICIQ-UI SF can be introduced as a reminder quality indicator for systematic follow-up of both new and previously diagnosed UI patients or patients at risk for UI. In the context of outcomes research we also recommend assessments or reassessments at consultations, in accordance with the general practice guideline on $\mathrm{UI}$, and at regular time intervals, even in the longer term. These should be conducted after $\mathrm{UI}$ has been diagnosed, when pads are prescribed, as well as for patients at risk for UI. Long-term assessment is especially important as UI is a chronic disorder with a natural tendency to increase over time. UI is often complicated by aspects of physical status such as co-morbidities, genetic factors, neuropathic conditions, trauma, associated causalities such as bowel conditions and pelvic organ prolapse. ${ }^{64-67}$ In addition, these factors can be further classified according to their suspected cause, such as limited mobility or pelvic floor dysfunction. These complications make it difficult to keep patients motivated for training in the long run. However, knowledge of these factors can be used to argue in favour of repeated interventions by a trained healthcare professional. Decision support tools for assessment or reassessment and specification of the diagnosis of $\mathrm{UI}$ and its severity, its risk and prognostic factors, and patients' desire for treatment should be included in registries to facilitate the systematic follow-up of UI patients and patients at risk for UI. ${ }^{63,64}$

In addition, it should be of interest to assess the burden of $\mathrm{UI}$ in the long term. This could be achieved by annual evaluations of the cost-effectiveness, based on UIrelated costs, $Q A L Y_{\text {societal, }}, \mathrm{QALY}_{\text {patient }}$ and ISLY, as used for the economic evaluation in our trial. Thus, UI-related costs and the EQ-5D should be assessed once a year, even in the long term.

Other relevant aspects to take into account when considering an outcomes study are gender and age. Women with UI were overrepresented in our surveys (chapter 2 and 3 ) as well as in our pragmatic trial (chapter 5), while the mean age of the patients in the trial was quite high, at 60 years. Although this imbalance might be to some extent inherent in the prevalence of the disorder, gender and age were 
found to play a role both in GPs' opinions about adhering to the UI guideline and in patient satisfaction with the UI care received. Therefore, future research with a more naturalistic recruitment approach should focus on these gender- and agespecific issues as well as on race and ethnicity issues as regards help-seeking behaviour, communication about available treatments and patient needs, to allow findings to be generalised to the overall adult primary care UI population in the Netherlands or elsewhere.

\section{Recommendations for healthcare practice}

The various studies in this project showed that involving nurse specialists produces results that are beneficial to all involved, and that the methods used seem suitable to implement optimal UI management in primary care. As mentioned in our overall conclusions, we therefore recommend adopting the intervention on a larger scale in primary care. However, several prerequisites need to be carefully considered, and potential barriers need to be tackled.

As a first prerequisite, the issue of recording $U I$ as a diagnosis is relevant. In our study we found that many GPs were unable to generate from their own ICPC coding system a complete list of their UI patients and the type of UI patients in their practice, nor whether referrals or prescriptions had been provided. Many GPs retrieved their UI patients through the records of pharmacies. There is thus an obvious need to improve awareness of UI in primary care as a serious chronic condition with considerable impact on patients and their caregivers and thus recording the diagnosis of UI, followed by a specification of the diagnosis in routine general practice. Once $\mathrm{UI}$ is being recorded, this will allow the systematic use and integration in routine electronic medical records of the simple and validated ICIQ-UI SF questionnaire to assess overall UI severity (frequency and amount of urine loss and impact of UI on $\mathrm{HRQOL}$ ), in combination with a validated instrument like the $3-1 \mathrm{Q}^{68}$ for the specification of UI diagnosis as a reminder and as a decision support tool. This can be a powerful way to improve diagnostic accuracy of $\mathrm{UI}$ and patient follow-up. This is especially important since all the questions of these questionnaires are basically the routine questions that should be asked according to GP guideline on in UI. ${ }^{45}$

A second prerequisite is that of patient monitoring and quality indicators. A potential quality indicator in routine care is the use of a bladder diary, as recommended in the GP guidelines on UI. Such a bladder diary can be a very practical tool to further specify the UI problem and to give guidance to treatment options and to monitor patients over time. Furthermore, quality indicators, or a reminder system, should be introduced when pads are prescribed. Incorporating these tools in existing electronic patient records can facilitate monitoring and systematic follow-up of $\mathrm{UI}$ patients and patients at risk for UI. 
As a third prerequisite, training of both nurses and GPs is essential. More nurses should be effectively trained to overcome their current limited availability and relative distance to GP practices.

In addition, nurses need regular supervision, feedback, financial resources and personal contacts with GPs about the protocol to be used.

Both nurses and GPs should be trained in effective communication skills, adapted to the age, gender, culture, language and socioeconomic features and strategies of the target group. Such skills may stimulate appropriate help-seeking behaviour in patients suffering from, or at risk for, UI. This could enable shared decision making through appropriate and timely information and a discussion of available UI treatments as well as patients needs and preferences for information or treatment. ${ }^{16,69,70}$

As a consequence, the involvement of nurse specialists will facilitate continence awareness in general, among UI patients and healthcare professionals alike. In view of the great burden that $\mathrm{UI}$ imposes on informal and formal caregivers as people age, nurse specialists and GPs might also be trained to play an important role in coaching care givers. This might not only improve the quality of care but also decrease care giver burden.

Another important prerequisite to be acknowledged is that the provision of continence care should largely depend on health services with a transparent and widely accepted organisation and infrastructure. There is currently wide variation in the accessibility and service capacity for UI patients. Currently, pelvic physiotherapists play an important role in service delivery to UI patients in primary care, next to continence nurses. However, $\mathrm{UI}$ is so widespread and affects so many different types of people that they may present for help to literally any healthcare professional. ${ }^{10,23,42-50}$ Consequently, involvement of trained nurse specialists in primary UI care could fit into a variety of contexts or models of care programmes in primary care that are all aimed at improving the quality of care. ${ }^{36,60}$ A nurse specialist, who is close to the GP (the first point of contact for most patients' health problems), can play a central role in timely identification of $\mathrm{UI}$, monitoring the effects of all $\mathrm{UI}$ treatments that are started, both in the short and the long term, and share experiences with the health professionals involved. To avoid controversies or conflicts as well as harm to patients, and to promote a wise and wide implementation of the intervention, we recommend that the boundaries of the nurses' professional responsibilities, their qualifications and competencies, should be clearly defined. Further, potential barriers to large-scale nurse specialist involvement include lack of knowledge and skills, lack of time for new health problems, lack of information and training for nurse specialists and limited financial support or lack of financial incentives and reimbursement for GPs, home care or hospital for nurse specialists. In our trial, hospital management, home-care management and a health insurer collaborated in organising the care and financing the nurse specialists. Now, as the study 
results are available, similar organisations will be informed and, whenever appropriate, included in the implementation and dissemination of the intervention.

Regional GP cooperatives and home-care institutions, in cooperation with hospital-linked pelvic care centres, should be involved to facilitate efficient large-scale recruitment of nurse specialists and $\mathrm{POH}$ for education and training. When adopting this role revision in UI primary care, policy makers will need to address the issues of the nursing service capacity required, the price of the product delivered and the implementation strategies to be used.

\section{Overall conclusion}

In view of the results of the various studies in this project on the causes of the burden of illness of UI and the effectiveness, efficiency and feasibility of involving trained nurse specialists in the primary care of UI patients, as well as the evidence from ongoing studies and the literature, we consider the involvement of such nurse specialists to be valuable. More specifically, based on our feasibility findings and the cost-effectiveness results, and taking into account that the implementation costs are likely to be low, we recommend adopting the nurse specialist intervention in primary care, accompanied by observational research. This implementation can only be successful if several conditions are met. Adopting nurse specialist interventions in routine practice offers opportunities to implement the 'monitoring and reassessment' steps of the TAIL approach in a sample, using a uniform monitoring system for all competing constructs of UI care. This chapter has offered methodological recommendations for such observational studies.

Such an observational study, in which nurse specialists support and substitute for GPs, as well as playing a central role in the timely identification of UI, monitoring the effects of all UI treatments started and sharing experiences especially with primary care pelvic physiotherapists and continence nurses might be called: 'Who really care? Aspects of primary care for UI.' In this way, the involvement of trained nurse specialists in the primary care of UI patients can lead to effective and efficient care and a lower burden of illness of UI. 


\section{References}

1. Health Council of the Netherlands (Gezondheidsraad). Urine Incontinentie [Urinary Incontinence]. Publication no. 2001/12. The Hague 2001. Report No.: ISBN: 90-5549-375-9.

2. Subak LL, Brown JS, Kraus SR, Brubaker L, Lin FM, Richter HE, et al. The "Costs" of Urinary Incontinence for Women. Obstet Gynecol. 2006;107(4):908-16.

3. Garrison Jr. L, Neumann P, Erickson P, al. e. Using real-world data for coverage and payment decisions: The ISPOR real-world data task force report. Value Health. 2007;10:326-35.

4. Tugwell P, Bennett KJ, Sackett D, L, Haynes RB. The measurement iterative loop: A framework for the critical appraisal of need, benefits and costs of health interventions. J Chron Dis. 1985;38(4):339-51.

5. Craig P, Dieppe P, Macintyre S, Michie S, Nazareth I, Petticrew M. Developing and evaluating complex interventions: the new Medical Research Council guidance. BMJ. 2008;337(1):a1655-.

6. Lewin S, Glenton C, Oxman AD. Use of qualitative methods alongside randomised controlled trials of complex healthcare interventions: methodological study. BMJ. 2009;339(1):b3496-.

7. Govier F, Litwiller S, Nitti V, Kreder KJ, Rosenblatt P. Percutaneous afferent neuromodulation for the refractory overactive bladder: Results of a multicenter study. J Urol. 2001;165:1193-8.

8. Viktrup L, Kent $H$, Summers D, Susan, L. Clinical practice guidelines on the initial assessment and treatment of urinary incontinence in women: a US focused review. Int J Gynecol Obstet. 2004;86(Supplement 1):S25-S37.

9. Milsom I, Altman D, Lapitan M, Nelson R, Sillén N, Thom D. Committee 1. Epidemiology of Urinary (UI) and Faecal (FI) Incontinence and Pelvic Organ Prolapse (POP). In: Abrams P, Cardozo L, Khoury S, Wein A, editors. Incontinence 4th International Consultation on Incontinence Paris July 5-8, 2008. Plymouth: Health Publication Ltd; 2009. p. 63, 72, 89-92.

10. Newman D, Ee C, Gordon D, Srini V, Williams K, Cahill B, et al. Committee 21. Continence Promotion, Education and Primary Prevention. In: Abrams P, Cardozo L, Khoury S, Wein A, editors. Incontinence 4th International Consultation on Incontinence Paris July 5-8, 2008. Plymouth: Health Publication Ltd; 2009.

11. James S, Daubert J-C, Werf F vd. Commentary: Use of registries to investigate the past and develop the future. BMJ. 2011;342.

12. Zwarenstein M, Treweek S, Gagnier JJ, Altman DG, Tunis S, Haynes B, et al. Improving the reporting of pragmatic trials: an extension of the CONSORT statement. BMJ. 2008;11(337):a2390.

13. Thorpe KE, Zwarenstein M, Oxman AD, Treweek S, Furberg CD, Altman DG, et al. A pragmatic explanatory continuum indicator summary (PRECIS): a tool to help trial designers. J Clin Epidemiol. 2009;62(5):464-75.

14. Teunissen D, Lagro-Janssen T. Urinary incontinence in community dwelling elderly: are there sex differences in help-seeking behaviour? Scand J Prim Health Care. 2004;22(4):209 - 16.

15. Teunissen TAM, Lagro-Janssen ALM. Sex differences in the use of absorbent (incontinence) pads in independently living elderly people: do men receive less care? Int J Clin Pract. 2009;63(6):869-73.

16. Shaw C, Brittain K, Tansey R, K. W. How people decide to seek health care: A qualitative study. Int J Nurs Stud. 2008;45(10):1516-24.

17. Klovning A, Avery K, Sandvik H, Hunskaar S. Comparison of two questionnaires for assessing the severity of urinary incontinence: The ICIQ-UI SF versus the incontinence severity index. Neurourol Urodynam. 2009;28(5):411-5.

18. Drummond M. Discussion: challenges in the economic evaluation of incontinence services. Urology. 1997;50(Supplement 6A):108.

19. DeLancey J. The hidden epidemic of pelvic floor dysfunction: Achievable goals for improved prevention and treatment. Am J Obstet Gynecol. 2005;192(5):1488-95.

20. Laurant M, Harmsen M, Faber M, Wollersheim H, Sibbald B, Grol R. Revision of professional roles and quality improvement: a review of the evidence. London: The Health Foundation. 2010. Contract No.: 6_suppl. 
21. Imamura M, Abrams P, Bain C, Buckley B, Cardozo L, Cody J, et al. Systematic review and economic modelling of the effectiveness and cost-effectiveness of non-surgical treatments for women with stress urinary incontinence. Health Technol Assess. 2010;14(40).

22. Veer de A, Francke A, Plas M, Vogel B. Goede loopbaanmogelijkheden belangrijk voor verpleegkundigen en verzorgenden. Factsheet Panel Verpleegkundigen en Verzorgenden: LEVV National Expertise Centre for Nursing and Care 2008.

23. Westert GP, Faber M. Commentary: the Dutch approach to unwarranted medical practice variation. BMJ. 2011;342.

24. Zwarenstein M, Treweek S, Gagnier JJ, Altman DG, Tunis S, Haynes B, et al. Improving the reporting of pragmatic trials: an extension of the CONSORT statement. BMJ. 2008;337:a2390.

25. Moher D, Hopewell S, Schulz KF, Montori V, Gotzsche PC, Devereaux PJ. CONSORT 2010 Explanation and Elaboration: updated guidelines for reporting parallel group randomised trials. BMJ. 2010;340:c869.

26. Council for Public Health and Health Care (RVZ). Het pakketprincipe kosteneffectiviteit. Achtergrondstudie ten behoeve van de 'appraisal' fase in pakketbeheer 2010. Contract No.: 291.

27. Avery K, Donovan J, Peters T, Shaw C, Gotoh M, Abrams P. ICI-Q: a brief and robust measure for evaluating the symptoms and impact of urinary incontinence. Neurourol Urodynam. 2004;23(4):322-30.

28. Gold M, Siegel J, Russell L. Cost-effectiveness in health and medicine. New York: Oxford University Press; 1996.

29. Drummond M, Sculpher M, Torrance G, O'Brien B, Stoddart G. Chapter 3. Critical assessment of economic evaluation. In: Drummond M, Sculpher M, Torrance G, O'Brien B, Stoddart G, editors. Methods for the economic evaluation of Heatlth Care Programmes. Third Edition ed. New York: Oxford University Press; 2005. p. 27-51.

30. Hulscher M, Laurant M, Grol R. Process evaluation on quality improvement interventions. Qual Saf Health Care. 2003;12(1):40-6.

31. Grol R, Wensing M. What drives change? Barriers to and incentives for achieving evidence-based practice. Med J Aust. 2004;180:S57-S60.

32. Grol R, Buchan H. Clinical guidelines: what can we do to increase their use? Med J Aust. 2006;185 (6):301-2.

33. Grol R, Bosch M, Hulscher M, Eccles M, Wensing M. Planning and Studying Improvement in Patient Care: The Use of Theoretical Perspectives. Milbank Q. 2007;85(1):93-138.

34. Hay-Smith J, Berghmans B, Burgio K, Dumoulin C, Hage S, Moore K, et al. Committee 12. Adult Conservative Management. In: Abrams P, Cardozo L, Khoury S, Wein A, editors. Incontinence 4th International Consultation on Incontinence Paris July 5-8, 2008. Plymouth: Health Publication Ltd; 2009.

35. Abrams P, Andersson KE, Birder L, Brubaker L, Cardozo L, Chapple C, et al. Fourth international consultation on incontinence recommendations of the international scientific committee: Evaluation and treatment of urinary incontinence, pelvic organ prolapse, and fecal incontinence. Neurourol Urodynam. 2010;29(1):213-40.

36. Health Council of the Netherlands (Gezondheidsraad). Role redefinition in health care. The Hague: Health Council of the Netherlands, 2008. Report No.: publication no. 2008/25.

37. Moore K, Wei Hu T, Subak L, Wagner T, Deutekom M. Committee 22. Economics of Urinary and Faecal Incontinence, and Prolapse. In: Abrams P, Cardozo L, Khoury S, Wein A, editors. Incontinence 4th International Consultation on Incontinence Paris July 5-8, 2008. Plymouth: Health Publication Ltd; 2009. p. 1708, 9.

38. Council for Public Health and Health Care (RVZ). Health 2.0 It's up to you. The Hague, February 2010: Advisory produced by the Council for Public Health and Health Care for the Minister of Health, Welfare and Sport 2010.

39. Knies S, Severens J, Ament A, Evers S. Using cost-effectiveness results from abroad for local policy decisions. Eur J Hosp Pharm 2008;14(4 ):51-4.

40. Health Insurance Board (CVZ). Urine Incontinentie bij volwassenen [Urinary Incontinence in adults]. Diemen 2003 Contract No.: 2003/147. 
41. Health Insurance Board (CVZ). Attentie voor incontinentie [Attention for incontinence]. Amstelveen 2006.

42. Stichting Bekkenbodem Patienten (SBP). 2001; Available from: http://www.bekkenbodem.net/.

43. Salzmann W, Reij R. Experiment Directe Toegankelijkheid Fysiotherapie. Sector Preventie, Paramedische Zorg en Tandheelkunde. Amstelveen: Health Insurance Board (CVZ)2002. Report No.: Cu401051.

44. Dutch Association for Physical Therapy for Pelvic Floor Disorders and Pre- and Postnatal Healthcare (NVFB). Functieprofiel Bekkenfysiotherapeut: Dutch Association for Physical Therapy for Pelvic Floor Disorders and Pre- and Postnatal Healthcare (NVFB) 2003.

45. Lagro-Janssen ALM, Teunissen D, Breedveldt-Boer HP, Dongen van J, Lemain TJJ, Steeneken F, et al. NHG-Standaard Incontinentie voor Urine M46 Eerste herziening [Dutch College Guideline on Urinary Incontinence. First revision]. Huisarts Wet. 2006;49(2):501-10.

46. Poot $\mathrm{E}$, Vaart van der $\mathrm{CH}$, Mulder $\mathrm{H}$. Urine-incontinentie bij vrouwen: het adapteren van een richtlijn. Nederlands Tijdschrift voor Evidence Based Practice. 2008;2:17-22.

47. Dielissen P, Jagt L vd, Lagro-Janssen T. Kaderhuisarts bouwt bruggen. Medisch Contact. 2009;64(38): 1593-5.

48. Faber E, Custers J, Berghmans L, Dongen van J, Groeningen van C, Grupping-Morel M, et al. Landelijke Eerstelijns Samenwerkings Afspraak Incontinentie voor Urine 'LESA' [National First Line Multidisciplinary Agreement]. Huisarts Wet. 2007;50(5):S9-S12.

49. Landelijk Expertisecentrum Verpleging \& Verzorging (LEVV). Richtlijn urine-incontinentie bij kwetsbare ouderen: 2010.

50. CV\&V. Beroepsdeelprofiel Continentiezorg. Utrecht: Afdeling Continentie Verpleegkundige en Verzorgenden (CV\&V) 2010.

51. Wensing M, Bosch M, Grol R. Developing and selecting interventions for translating knowledge to action. CMAJ. 2010;182(2):E85-8.

52. Du Moulin MFMT, Hamers JPH, Paulus A, Berendsen C, Halfens R. The role of the nurse in community continence care: a systematic review. Int J Nurs Stud. 2005 5;42(4):479-92.

53. Williams KS, Assassa RP, Cooper NJ, Turner DA, Shaw C, Abrams KR, et al. Clinical and costeffectiveness of a new nurse-led continence service: a randomised controlled trial. $\mathrm{Br} J$ Gen Pract. 2005;55(518):696-703.

54. Williams KS, Assassa RP, Gillies CL, Abrams KR, Turner DA, Shaw C, et al. A randomized controlled trial of the effectiveness of pelvic floor therapies for urodynamic stress and mixed incontinence. BJU Int. 2006;98(5):1043-50.

55. Du Moulin MFMT, Hamers JPH, Paulus A, Berendsen CL, Halfens R. Effects of Introducing a Specialized Nurse in the Care of Community-Dwelling Women Suffering From Urinary Incontinence: A Randomized Controlled Trial. J Wound Ostomy Continence Nurs. 2007;34(6):631-40.

56. Bodenheimer T, Chen E, Bennett D. Confronting The Growing Burden Of Chronic Disease: Can The U.S. Health Care Workforce Do The Job? Health Aff. 2009;28(1):64-74.

57. Lagro-Janssen A, Teunissen D. An educational and training course of urinary incontinence primary care management for practice nurses (POH). Nijmegen 2009.

58. Theel F. De inzet van praktijkondersteuners bij Incontinentieproblemen. Nieuwe aanpak lijkt te werken. De POH Tijdschrift voor en door praktijkondersteuners. 2010;3.

59. Berghmans B, Winkens R. 'Continentiezorg'. An educational and training course of urinary incontinence primary care management for nurse specialists. Maastricht 2010.

60. Beusmans G, Crebolder HF, Ree van J. Zorg voor Chronisch zieken: Praktijkverpleegkundigen breed inzetten. Medisch Contact. 2001;56(7):259-61.

61. Grimshaw J, Thomas R, MacLennan G, Fraser C, Ramsay C, Vale L, et al. Effectiveness and efficiency of guideline dissemination and implementation strategies. Health Technol Assess. 2004;8(6):1-72.

62. O'Cathain A, Murphy E, Nicholl J. Three techniques for integrating data in mixed methods studies. BMJ. 2010;341(c4587). 
63. Kievit W, Fransen J, Adang EM, den Broeder AA, Bernelot Moens HJ, Visser H, et al. Long-term effectiveness and safety of TNF-blocking agents in daily clinical practice: results from the Dutch Rheumatoid Arthritis Monitoring register. Rheumatology (Oxford). 2011;50(1):196-203.

64. Landefeld CS, Bowers BJ, Feld AD, Hartmann KE, Hoffman E, Ingber MJ, et al. National Institutes of Health State-of-the-Science Conference Statement: Prevention of Fecal and Urinary Incontinence in Adults. Ann Intern Med. 2008;148(6):449-58.

65. Slieker-ten Hove M, Pool-Goudzwaard A, Eijkemans M, Steegers-Theunissen R, Burger C, Vierhout M. The prevalence of pelvic organ prolapse symptoms and signs and their relation with bladder and bowel disorders in a general female population. Int Urogynecol J. 2009;20(9):1037-45.

66. Slieker-ten Hove MCP, Pool-Goudzwaard AL, Eijkemans MJC, Steegers-Theunissen RPM, Burger CW, Vierhout ME. Symptomatic pelvic organ prolapse and possible risk factors in a general population. Am J Obstet Gynecol. 2009;200(2):184.e1-.e7.

67. Slieker-ten Hove MCP, Pool-Goudzwaard AL, Eijkemans MJC, Steegers-Theunissen RPM, Burger CW, Vierhout ME. Prevalence of double incontinence, risks and influence on quality of life in a general female population. Neurourol Urodynam. 2010;29(4):545-50.

68. Brown JS, Bradley CS, Subak LL, Richter HE, Kraus SR, Brubaker L, et al. The Sensitivity and Specificity of a Simple Test To Distinguish between Urge and Stress Urinary Incontinence. Ann Intern Med. 2006;144(10):715-23.

69. Harris M, McKenzie S. Mens's health: what's a GP to do? Med J Aust. 2006;185(8):440-4.

70. Goode PS, Burgio KL, Richter HE, Markland AD. Incontinence in Older Women. JAMA. 2010;303(21): 2172-81. 


\section{Summary}


This thesis describes the evaluation of various aspects of involving nurse specialists in primary care for urinary incontinence (UI). The main objective of the studies presented was to examine whether the availability and involvement of a nurse specialist in primary UI care is feasible and leads to better effects on patient outcomes and to cost-effective care for adult patients with UI, as compared to care-as-usual. We used the seven steps of Tugwell's 'technology assessment iterative loop' (TAIL) approach to guide our choice of quantitative and qualitative research methods for the evaluation of the various aspects of primary care for UI patients, with the aim of providing input for decision making in policy and practice.

The general introduction of this thesis, chapter $\mathbf{1}$, discusses the background to and relevance of the studies and introduces the research questions. Doubts about the quality, efficiency and organisation of care for $\mathrm{UI}$ and the rising socio-economic burden imposed by UI on the healthcare system have made it increasingly important to find solutions to improve all aspects of UI care. The chapter gives an overview of the burden of illness presented by $\mathrm{UI}$, and introduces and identifies some of the risk factors and prognostic factors, as well as the role of help-seeking behaviour, as potential causes of the burden of UI, and discusses potentially feasible and effective interventions to reduce this burden.

National and international UI guidelines indicate that conservative management, with appropriate advice about potentially modifiable lifestyle factors, together with bladder and pelvic floor muscle training, and about the efficient use of absorption pads, is the main approach to initial care for $\mathrm{UI}$ in both men and women. All of these require changes of behaviour and long-term adherence. Despite the availability of clinical practice guidelines on UI for general practitioners (GPs), and since it seems that many UI patients have pads prescribed to them by their GP without adequate diagnostics or treatment, there are serious doubts about the dissemination and implementation of clinical practice guidelines for UI.

In 2001, a committee of the Dutch Health Council concluded that there is insufficient knowledge about typical primary UI care, and that no information is available about adherence to existing guidelines on UI care in routine practice. Since 2002, there has been a growing emphasis on involving nurse specialists in general practice care, with favourable findings for chronic disorders. Revision of professional roles is seen as a viable strategy to consider when addressing the challenges facing healthcare over the coming decades.

When we started our research in this area, one systematic literature review was available on role revision from GPs to nurse specialists in UI care, and although the evidence was limited and weak, we concluded that nurse specialists might be well accepted by patients and GPs, and could represent a feasible and useful option to change the organisation of UI care to achieve effective and efficient UI management. Arguments for the involvement of nurse specialists in UI care are that nurse specialists have specific expertise, are used to following protocols, spend more time 
motivating patients, are familiar with emotional support for patients and their informal caregivers regarding behavioural change, and offer a low-threshold service. Moreover, $\mathrm{UI}$ is not always a straightforward condition, for several reasons: there is a wide variation in help-seeking behaviour; UI is frequently complicated by pelvic floor related dysfunctions (e.g. pelvic organ prolapse, faecal incontinence) and comorbidities, particularly among the elderly; and if not treated and monitored, $\mathrm{UI}$ is a chronic, not self-limiting, dynamic condition with a strong tendency to worsen over time. After additional training in UI care - modelled on that for nurse specialists for diabetes, chronic pulmonary diseases and cardiovascular risk management - nurse specialists may therefore be well equipped to support GPs. This may offer a solution to the current inadequate $\mathrm{UI}$ care, including the inappropriate prescribing of incontinence pads.

In view of the paucity of available data on typical UI care for adults in primary care, Part I of this thesis examines important issues for GPs and patients in UI care, to identify barriers and facilitators for the improvement of the efficacy, acceptability and quality of UI care. We describe the results of two cross-sectional postal surveys (one among GPs and one among adult UI patients) which we performed in two Dutch regions to examine the current care-as-usual for adults with UI in Dutch general practice.

Chapter 2 presents the findings of the survey among 264 Dutch GPs. We report on their adherence levels and their reasons for adherence or non-adherence to the Guideline on Urinary Incontinence of the Dutch College of General Practitioners. GPs were found to incompletely adhere to their guideline on UI care in terms of diagnosis and treatment: compliance with diagnostic recommendations was fairly good, although a bladder diary was not often used (35\%); compliance with treatment recommendations was low: adherence to therapeutic procedures was only high for mild/moderate stress UI. One out of four GPs reported that adhering to the guideline on UI care is difficult, mainly due to lack of time, staff, diagnostic tools and competencies to provide this care, and due to low motivation among patients. We conclude that there is room to improve the implementation of guidelines on UI. Since effective management of UI by GPs is time-consuming, especially in the case of more complex problems such as severe stress UI, urgency $\mathrm{UI}$ and mixed UI, or concurrent co-morbidities, further research should focus on solutions to tackle major barriers and to introduce facilitators, for instance substitution of tasks to specialised nurses, reducing the threshold for referral and concentrating expertise in integrated continence care services.

Chapter 3 describes the results of the survey - performed in the same areas and the same period as the GP survey - among 208 Dutch adult UI patients who had re- 
ceived a first-time prescription for incontinence pads in the preceding year. Care-asusual was studied in this specific patient group because it is often assumed that pads are necessary and they are prescribed in addition to the recommended/necessary treatment. We report on the further treatment that the UI patients had received from their GP apart from pads, and their satisfaction with the GP care they had received. One out of three patients reported that they had had no direct contact with their GP for their first pad prescription, and half of the $147 \mathrm{UI}$ patients who had consulted their GP had only had pads prescribed. One out of four $\mathrm{UI}$ patients was not satisfied with the information on $\mathrm{UI}$ they had received from their GP; this was especially reported by younger patients, women, less healthy patients and patients with urgency UI. Overall, we conclude that Dutch UI patients using pads experience suboptimal and unsatisfactory GP care and management and that factors identified through patient feedback should be used to improve the quality of care for UI in primary care.

The findings of the studies in part I on Dutch care-as-usual confirmed that GP care and management for adult UI patients is substandard, with inappropriate prescribing of incontinence pads. Hence, and considering the factors identified by GPs and UI patients, these studies underline the relevance of the various aspects of involving specially trained nurse specialists in primary care for adult patients with $U I$ that we studied in Part II of our research project, as a possible solution for the inadequate UI care in primary care.

The studies presented in Part II are all based on a multicentre pragmatic randomised controlled trial (RCT) of adults with stress, urgency or mixed UI, called Promoting Continence Study (PromoCon Study), performed between 2005 and 2008 in four Dutch regions (Maastricht, Nijmegen, Helmond, The Hague), comparing a one-year intervention by six trained home-care and hospital-linked nurse specialists with care-as-usual, after initial diagnosis and assessment by GPs.

Chapter 4 describes the study protocol of the PromoCon project. The main objective of the project was to investigate whether the availability and involvement of nurse specialists in a new role as a substitutes for GPs in the management of UI in general practice would lead to more efficient care for adult UI patients. To this end, the project consisted of an effectiveness evaluation (Chapter 5), an economic evaluation from the societal perspective (Chapter 6) and evaluations of the feasibility of the intervention (Chapters $\mathbf{7}$ and 8). The research questions, the study design, primary and secondary outcomes, data collection methods and timing and data analysis are described and discussed. The intervention by the nurse specialists was conducted in the context of a pragmatic prospective multicentre two-armed RCT among patients with $\mathrm{UI}$ in general practices in four regions in the Netherlands. The design of the nurse intervention protocol, based on clear and accepted work agree- 
ments/protocols in accordance with the UI guideline for GPs, is described and discussed. After initial medical UI diagnosis by the GP, the nurse specialist takes over tasks from the GP that relate to specification of the diagnosis, intervention and monitoring of patients with the most prevalent types of UI (stress, urgency and mixed UI). The GP retains the final responsibility. The one-year intervention consisted of five to seven visits during the first three months, followed by consultations at six and 12 months to monitor effect and adherence. The training programme and the competence assessment of the nurse specialists prior to the trial are described. The main goal of the intervention by the nurse specialist is to provide a tailored, patient-specific diagnostic and treatment plan, thereby preventing or reducing the use of incontinence pads. Based on the primary outcome data on the severity of UI (measured with the International Consultation on Incontinence Questionnaire Short Form [ICIQ-UI SF]), the number of patients we needed to include was 350 . The economic evaluation involved measurements of quality of life and costs alongside the clinical trial. All consecutive adult patients consulting their GP for stress, urgency or mixed UI over a one-year period, as well as patients previously diagnosed with these types of UI, were eligible for the study. Included patients were followed for 12 months.

Chapter 5 reports on the effectiveness of the intervention by nurse specialists in terms of the patient-reported outcome of UI severity, including symptoms of frequency and volume and the impact of UI on health-related quality of life (ICIQ-UI $\mathrm{SF})$, after three months and after one year, as studied in the PromoCon trial. The analysis was based on intention-to-treat principles. A total of 109 GPs in 65 practices participated, and 384 patients met the inclusion criteria; 186 were randomly allocated to the intervention and 198 to care-as-usual. The population predominantly consisted of women (93\%), with a mean age of 65 , and the groups were comparable. Of the 186 patients randomised to the nurse specialists, 137 (73.6\%) were treated according to protocol (seven to nine consultations). Cases where fewer consultations than prescribed by the protocol had been provided were related to poor health $(n=14)$ or lack of motivation/too much burden ( $n=15)$. Although no differences in terms of the one-year linear trend were found between the groups, patients within both study groups improved significantly in terms of UI severity and impact on UI-specific health-related quality of life at three months, as well as in terms of the one-year linear trend (both end-points $p<0.001$ ). However, after correction for effect modifiers (UI type, body mass index) we found significant differences between groups in favour of the intervention group at three months $(p=$ 0.04). After one year, no differences were found in terms of the one-year linear trend $(p=0.15)$. However, patients in the intervention group who had no anxiety/depression at baseline had improved significantly more than those in the careas-usual group ( $p=0.03$ ). We concluded that involving nurse specialists in the care 
for UI patients supplementary to that provided by general practitioners can reduce the severity and impact of UI, after correction for effect modifiers. This is also true for specific circumstances such as anxiety/depression. As UI is a chronic dynamic disorder and is often complicated by co-morbidities, the positive effect of the nurse specialist intervention in the short term would argue in favour of repeat interventions by trained healthcare professionals to monitor the condition and achieve longterm effects. We recommend that further research should focus on tailoring the intervention to those patient characteristics which are prognostic and predictive of $\mathrm{UI}$ and are modifiable (depression, overweight).

Chapter 6 presents the results of the economic evaluation implemented alongside the RCT to determine the 12-month societal cost-effectiveness of involving UI nurse specialists in primary care compared to care-as-usual by GPs. The for quality of life adjusted life year (QALY) and a urinary specific measure were used as outcome measures. The EuroQol-5D was used to calculate two different QALYs: a QALY based on preferences of the general population for health outcome (QALY societal) and the $\mathrm{QALY}_{\text {patient }}$ based on patient preferences for health outcome. In addition, the patient-reported sum score of severity and impact of UI of the ICIQ-UI SF is used to calculate a for the severity of UI weighted life year (ISLY). Healthcare resource use, patient and family costs, and productivity costs were assessed. Cost prices were based on Dutch standard tariffs and on own cost price research. Data were collected by three-monthly questionnaires. Incremental cost-effectiveness ratios (ICERS) were calculated by dividing the difference in the groups in costs by the difference measured on the different outcome measures. In general in the Netherlands an intervention is considered cost-effective when the incremental cost/QALYs ocietal $_{\text {al }}$ are below the threshold of $€ 40,000$. Uncertainty was assessed using bootstrap simulation. In addition, the upper limit of the value of further research to reduce uncertainty was calculated (the socalled Expected Value of Perfect Information). We found that, compared to care-as-usual, involving nurse specialists cost $€ 16,742$ per QALY societal gained. Thus far below the threshold value. However, the decision uncertainty was considerable. The probability that the intervention will be cost-effective is only $58 \%$. Consequently the expected value of perfect information is substantial: $€ 78$ million. Both $Q A L Y_{\text {patient }}$ and ISLY yielded slightly more favourable cost-effectiveness results with less uncertainty. The ICERs were considerably lower when the costs of the intervention were based on practice nurses instead of nurse specialists. The subgroup analysis showed that for UI patients without reported anxiety/depression, the probability that the nurse specialist intervention is cost-effective is considerably higher (69\%). Based on our cost-effectiveness results and taking into account that the implementation costs are likely to be minor, we recommend adopting the nurse specialist intervention in primary care, while conducting further research to reduce uncertainty. Careful monitoring of the performance of the intervention with suit- 
able recording of real world data will allow for longer follow-up with future evidence development and will yield further information for coverage decisions.

In addition to investigating the effectiveness and cost-effectiveness of nurse specialist involvement, we tried to understand what causes the effect, especially as we foresaw possible difficulties with the implementation of our intervention, given the 2006 reforms of the Dutch healthcare insurance system. Although role revision from GPs to nurses is increasing, we did not know how the nurses experienced their task and how consumers (i.e. patients and GPs) perceived and experienced the care provided by this new type of healthcare professional. This relates to our third aim, namely examining the feasibility of the intervention. To this end, we performed a qualitative focus group study (Chapter 7) with the nurse specialists who participated in the trial, to explore how they perceived their role in the care for UI patients and how they had performed the intervention. In addition, a postal questionnaire was sent to the UI patients to assess patient satisfaction in relation to relevant themes in the care for UI patients according to UI guidelines (Chapter 8). Finally, a convenience sample of the participating GPs were interviewed after the study in 10-minute structured interviews by telephone, to explore their experiences with and attitudes towards the intervention by nurse specialists, and their views on the value of this new form of care (Chapter 8). These studies were intended to explain potential effects and to identify facilitators and barriers for wider implementation.

Chapter 7 reports on the experiences and attitudes of the nurse specialists. In a qualitative focus group study, we explored experiences and attitudes of the six nurse specialists who had provided the intervention, regarding their role in the care for patients with UI, thereby identifying facilitators and barriers for wider implementation. We found that the nurse specialists felt competent to provide advice and information, to offer possible solutions and to monitor and guide the process of care for people with UI. They felt appreciated by patients and felt they had offered added value to the usual care by GPs. Nurses sometimes noticed that GPs lacked interest in UI. Important preconditions for effective nurse specialist care were personal contact with the GPs and the availability of enough time, sufficient equipment and financial resources. Nurse specialists valued continued education and feedback in the routine care for patients with UI. We conclude that trained nurse specialists appear to feel competent and satisfied about supporting GPs in the care for patients with UI and that they feel highly appreciated by both patients and GPs. In addition, these findings support the increasing involvement of nurse specialists in Dutch primary care and contribute to the knowledge about the feasibility of this type of care provision by nurse specialists, both from an international and national perspective. 
Chapter 8 describes satisfaction among consumers, that is, patients and GPs, about the care provided by this new type of healthcare professional. Patients' satisfaction with the care provided by either nurse specialists or GPs was measured with a selfcompleted questionnaire. GPs' views on the involvement of nurse specialists were measured in a structured telephone interview. The patient satisfaction score on the care offered by nurse specialists was 8.4 (scale 1-10), versus 6.4 for care-as-usual by GPs. Over $85 \%$ of patients would recommend nurse specialist care to their best friends and $77 \%$ of the GPs considered the role of the nurse specialist to be beneficial, giving it a mean score of 7.2. We conclude that substituting UI care from GP to nurse specialist is welcomed by both patients and GPs. Small changes like giving additional UI-specific information and devoting more attention to UI (which had been given little attention before), would provide a simple instrument to stimulate patient to change their behaviour in the right direction.

Finally, Chapter 9, the general discussion, presents a brief overview of the background and research objectives of the various studies presented in this thesis. The main findings are summarised, explained and combined into an overall conclusion. Methodological strengths and limitations are discussed with regard to the overall approach of our research project, the different quantitative and qualitative methods that we used to evaluate the construct of UI care and the interpretation of the findings. The chapter also presents implications and recommendations for research and healthcare practice. Overall, we conclude from the results of the various studies in this project, as well as the evidence from ongoing studies and the literature, that the involvement of such nurse specialists can be regarded as valuable.

More specifically, based on our feasibility findings and the cost-effectiveness results, and taking into account that the implementation costs are likely to be low, we recommend adopting the nurse specialist intervention in primary care, accompanied by observational research. This implementation can only be successful if several conditions are met. Adopting nurse specialist interventions in routine practice offers opportunities to implement the 'monitoring and reassessment' steps of the TAIL approach in a sample, using a uniform monitoring system for all competing constructs of UI care. The chapter offers methodological recommendations for such observational studies.

Such an observational study, in which nurse specialists support and substitute for GPs, as well as play a central role in the timely identification of UI, monitoring the effects of all UI treatments started and sharing experiences especially with primary care pelvic physiotherapists and continence nurses, might be called: 'Who really cares? Aspects of primary care for UI.' Such studies could help ensure that the involvement of trained nurse specialists in the primary care of UI patients can lead to effective and efficient care and a lower burden of illness due to UI. 
Samenvatting 
Dit proefschrift beschrijft de evaluatie van verschillende aspecten van het inzetten van gespecialiseerde verpleegkundigen ter ondersteuning van de huisarts bij de zorg voor volwassenen met klachten van ongewild urineverlies, oftewel urine incontinentie (UI). Het hoofddoel van de gepresenteerde studies was te onderzoeken of de beschikbaarheid en de inzet van een gespecialiseerd verpleegkundige in de eerste lijn haalbaar is en leidt tot betere effecten voor volwassenen met UI en tot kosteneffectieve UI zorg vergeleken met de bestaande zorg. In dit project ter evaluatie van de verschillende aspecten van de eerstelijns UI zorg hebben wij ons bij de keuze voor de kwantitatieve en kwalitatieve onderzoeksmethoden laten leiden door de zeven stappen van Tugwell's 'technology assessment iterative loop' (TAIL) aanpak. Ons doel was daarbij om input te geven voor besluitvorming voor beleid en voor de praktijk.

In de algemene inleiding van dit proefschrift, hoofdstuk 1, worden achtergrond en relevantie van de studies besproken en worden de onderzoeksvragen geïntroduceerd. Twijfels over de kwaliteit, efficiëntie en organisatie van de UI zorg en de toenemende sociaal-economische druk op de gezondheidszorg door UI, hebben het steeds belangrijker gemaakt om oplossingen te vinden ter verbetering van alle aspecten van de UI zorg. Het hoofdstuk geeft een overzicht van de ziektelast veroorzaakt door UI, en introduceert en identificeert enkele van de risicofactoren en prognostische factoren voor UI, alsook de rol die het hulpzoekgedrag speelt, als mogelijke oorzaken van de ziektelast door UI. Tevens worden potentieel haalbare en effectieve interventies besproken om deze ziektelast te verminderen.

Nationale en internationale richtlijnen op het gebied van UI geven aan dat de belangrijkste initiële benadering voor zowel mannen als vrouwen met $\mathrm{UI}$ bestaat uit conservatieve behandeling, met een passend advies voor beïnvloedbare leefstijlfactoren in combinatie met blaastraining en bekkenbodemspiertraining, en adviezen voor het efficiënt gebruik van absorptiematerialen. Deze conservatieve behandeling vraagt om gedragsverandering en langdurige therapietrouw. Ondanks het bestaan van UI-standaarden voor huisartsen, en omdat de indruk bestaat dat veel UI patiënten van hun huisarts incontinentiemateriaal krijgen voorgeschreven zonder adequate diagnostiek of behandeling, zijn er ernstige twijfels over de adequate verspreiding en implementatie van klinische praktijkrichtlijnen voor de behandeling van UI.

In 2001 concludeerde een commissie van de Nederlandse Gezondheidsraad dat er onvoldoende kennis bestaat over de gebruikelijke zorg voor UI in de eerste lijn, en dat er geen informatie over bestaat in hoeverre de bestaande richtlijnen voor UI in de dagelijkse praktijk worden opgevolgd door huisartsen. Sinds 2002 is er een groeiende belangstelling voor het inzetten van gespecialiseerde verpleegkundigen als ondersteuning in de huisartsenzorg, en voor wat betreft chronische aandoeningen zijn hiermee positieve ervaringen opgedaan. Taakherschikking van artsen naar niet-artsen, zoals bijvoorbeeld verpleegkundigen, en herziening van de rol van de verschillende zorgverleners, wordt gezien als een haalbare strategie om te overwe- 
gen bij de aanpak van de uitdagingen waar de gezondheidszorg de komende decennia voor komt te staan.

Bij de start van ons onderzoeksproject in 2005, was er één systematisch literatuuronderzoek voorhanden naar taakherschikking van huisarts naar verpleegkundig specialist op het gebied van de incontinentiezorg, en hoewel het wetenschappelijk bewijs beperkt en mager was, concludeerden we dat de inzet van getrainde gespecialiseerd verpleegkundigen acceptabel is zowel voor patiënten als huisartsen. Hun inzet zou een haalbare en bruikbare strategie kunnen zijn om de organisatie van de zorg voor UI om te buigen naar het effectiever en efficiënter managen van de zorg voor UI in de huisartsenpraktijk. Er zijn meerdere argumenten om gespecialiseerd verpleegkundigen te betrekken bij de incontinentiezorg. Dergelijke verpleegkundigen hebben hun specifieke expertise, zijn gewend om volgens protocollen te werken, besteden meer tijd aan het motiveren van patiënten, zijn vertrouwd met emotionele ondersteuning van zowel patiënten als hun mantelzorgers waar het gaat om gedragsverandering, en bieden laagdrempelige zorg. Bovendien is UI vaak een complexe aandoening, om diverse redenen. Allereerst is er een grote variatie in het hulpzoekgedrag van mensen met UI. Daarnaast gaat UI vaak samen met andere stoornissen die te maken hebben met een dysfunctie van de bekkenbodem -zoals bijvoorbeeld verzakkingen en ontlastingsproblemen als obstipatie en ongewild ontlastingsverlies- en co-morbiditeiten, met name onder de ouderen. Tenslotte is UI, wanneer deze niet wordt behandeld en gemonitord, een chronische aandoening, waarvan de ernst en hinder in de loop der tijd kunnen variëren, die vaak niet vanzelf overgaat en die een sterke neiging heeft in ernst toe te nemen met het stijgen van de leeftijd. Gespecialiseerd verpleegkundigen zouden, na een aanvullende opleiding voor UI zorg volgens een model dat vergelijkbaar is met dat van gespecialiseerd verpleegkundigen voor diabetes, chronische long-, hart- en vaatziekten, goed in staat kunnen zijn om huisartsen te ondersteunen. Dit zou een oplossing kunnen bieden voor de huidige onvoldoende UI zorg, inclusief het niet adequaat voorschrijven van incontinentiemateriaal.

Gezien het gebrek aan data over de gebruikelijke dagelijkse zorg voor volwassenen met UI in de eerste lijn, is het doel van deel I van dit proefschrift het identificeren van de thema's die belangrijk zijn voor zowel huisartsen als UI patiënten met betrekking tot de UI zorg. Hiermee wordt beoogd inzicht te geven in de belemmerende en bevorderende factoren voor de verbetering van de effectiviteit, de aanvaardbaarheid en de kwaliteit van de UI zorg. Beschreven worden de resultaten van twee cross-sectionele schriftelijke vragenlijstonderzoeken (één onder huisartsen en één onder volwassen patiënten met UI), die we hebben uitgevoerd in twee Nederlandse regio's met als doel de huidige gebruikelijke zorg voor volwassenen met $\mathrm{UI}$ in de Nederlandse huisartsenpraktijk te bestuderen. 
In Hoofdstuk 2 worden de resultaten van het vragenlijstonderzoek onder $264 \mathrm{Ne}$ derlandse huisartsen gepresenteerd, en wordt verslag uitgebracht over de mate waarin zij de huisartsenstandaard M46 voor UI al dan niet naleven en wat hun redenen daarvoor zijn. Huisartsen bleken de standaard UI slechts gedeeltelijk na te leven voor wat betreft diagnostiek en therapie: naleving van de diagnostische aanbevelingen was redelijk goed, hoewel niet vaak een mictiedagboek werd gebruikt (35\%); naleving van de aanbevelingen voor behandeling was gering: de naleving van therapeutische procedures was alleen goed voor lichte / matige stress UI. Eén op de vier huisartsen rapporteerde dat het naleven van de UI standaard vooral lastig is door een gebrek aan tijd, personeel, diagnostische instrumenten en competenties om deze zorg te kunnen geven, en vanwege een lage motivatie bij de patiënten. We concludeerden dat er ruimte is voor verbetering van de implementatie van UI richtlijnen. Omdat effectieve UI zorg door huisartsen tijdrovend is, vooral wanneer er sprake is van meer complexe problematiek, zoals ernstige stress UI, urgency UI en gemengde UI, of bij co-morbiditeit, zou verder onderzoek zich moeten richten op oplossingen voor het aanpakken van grote belemmeringen en het introduceren van bevorderende factoren, zoals substitutie van taken van de huisarts door gespecialiseerd verpleegkundigen, het verlagen van de drempel om UI patiënten door te verwijzen en het concentreren van expertise in gespecialiseerde multidisciplinaire centra voor continentiezorg.

Hoofdstuk 3 beschrijft de resultaten van het vragenlijstonderzoek - uitgevoerd in dezelfde regio's en in dezelfde periode als het vragenlijstonderzoek onder huisartsen - onder 208 volwassenen met UI, die in het jaar voorafgaand aan het onderzoek voor de eerste keer incontinentiemateriaal op recept hadden ontvangen. Care-asusual werd in deze specifieke groep patiënten onderzocht, omdat vaak wordt aangenomen dat incontinentiemateriaal noodzakelijk is en voorgeschreven wordt naast de aanbevolen / noodzakelijke behandeling. Onderzocht werd welke zorg UI patiënten van hun huisarts kregen naast het voorschrift voor incontinentiemateriaal, en hoe tevreden zij waren over deze zorg. Eén op de drie patiënten meldde geen direct contact met hun huisarts te hebben gehad vóór hun eerste recept voor incontinentiemateriaal. De helft van de 147 UI patiënten die wel direct contact hadden gehad met hun huisarts, had alleen een recept voor incontinentiemateriaal gekregen. Eén op de vier patiënten was niet tevreden over de informatie die zij van hun huisarts hadden ontvangen met betrekking tot hun urineverlies. Dit werd met name gerapporteerd door de jongere patiënten, vrouwen, minder gezonde patiënten en patiënten met urgency UI. De algemene conclusie is dat volwassenen in Nederland die incontinentiemateriaal krijgen voorgeschreven via de huisarts, de zorg voor hun UI als suboptimaal ervaren en daar niet tevreden over zijn, en dat factoren die via deze feedback van patiënten geïdentificeerd zijn, gebruikt moet worden om de kwaliteit van de zorg voor UI in de eerste lijn te verbeteren. 
De bevindingen van de studies in deel I over de gebruikelijke Nederlandse UI zorg bevestigen dat de huisartsenzorg voor volwassenen met UI substandaard is, en wordt gekenmerkt door onterecht voorschrijven van incontinentiemateriaal. Daarmee vormen deze studies, mede gezien de factoren die geïdentificeerd zijn door huisartsen en UI patiënten, een ondersteuning voor de relevantie van de verschillende in deel II van dit project bestudeerde aspecten van de inzet van speciaal opgeleide gespecialiseerd verpleegkundigen in de eerste lijn voor volwassenen met UI. Doel hiervan was te komen tot een mogelijke oplossing voor de inadequate UI zorg in de eerste lijn.

Alle studies die worden gepresenteerd in deel II zijn gebaseerd op een multicenter pragmatische gerandomiseerde gecontroleerde trial (RCT) onder volwassenen met stress, urgency of gemengde UI, genaamd 'Promoting Continence Study' (PromoCon Study). Deze trial is uitgevoerd tussen 2005 en 2008 in vier Nederlandse regio's (Maastricht, Nijmegen, Helmond, Den Haag). Na de eerste diagnose en beoordeling door de huisarts werd hierbij een interventie van één jaar door zes getrainde gespecialiseerd verpleegkundigen vergeleken met de gebruikelijke zorg door de huisarts.

Hoofdstuk 4 beschrijft het studieprotocol van het PromoCon project. De belangrijkste doelstelling van het project was te onderzoeken of de beschikbaarheid en de inzet van gespecialiseerd verpleegkundigen in een nieuwe rol, waarbij taken in de UI zorg worden overgenomen van huisartsen, zou leiden tot een efficiëntere zorg voor volwassenen met UI. Om dit te onderzoeken bestond het project uit een effectevaluatie (hoofdstuk 5), een economische evaluatie vanuit maatschappelijk perspectief (hoofdstuk 6) en evaluaties van de haalbaarheid van de interventie (hoofdstuk 7 en 8). De onderzoeksvragen, de onderzoeksopzet, de primaire en secundaire uitkomstmaten, de dataverzamelingsmethoden en de meetmomenten en data-analyse worden beschreven en besproken. De interventie door de gespecialiseerd verpleegkundigen werd uitgevoerd in een pragmatische prospectieve multi-center tweearmige gerandomiseerde gecontroleerde trial bij patiënten met UI in huisartspraktijken in vier regio's in Nederland. De opzet en inhoud van het protocol van de interventie door de gespecialiseerd verpleegkundige, gebaseerd op heldere en geaccepteerde werkafspraken en protocollen overeenkomstig de huisartsenstandaard voor UI, wordt beschreven en besproken. Nadat in eerste instantie de huisarts de medische diagnose UI had gesteld, nam de gespecialiseerd verpleegkundige taken van de huisarts over met betrekking tot het nader specificeren van de diagnose, het uitvoeren van de interventie en het monitoren van patiënten met de meest voorkomende vormen van UI (stress, urgency en gemengde UI). De huisarts bleef eindverantwoordelijk. De interventie duurde één jaar en bestond uit vijf tot zeven bezoeken tijdens de eerste drie maanden, gevolgd door een consult na zes en 12 maanden om zowel het effect als het opvolgen van adviezen te monitoren. Het 
opleidingsprogramma van de verpleegkundigen en het beoordelen van hun competenties voorafgaand aan de trial worden beschreven. Het belangrijkste doel van de interventie door de gespecialiseerd verpleegkundige was het opstellen en uitvoeren van een op maat gesneden, patiënt-specifiek diagnostisch- en behandelplan om daarmee te komen tot een lager of meer passend gebruik van incontinentiemateriaal. Op basis van de primaire uitkomstmaat, de ernst van de UI (gemeten met de International Consultation Questionnaire for Urinary Incontinence Short Form [ICIQUI SF]), moesten wij minstens 350 patiënten includeren. Voor de economische evaluatie werden kwaliteit van leven en kosten tijdens de klinische trial bepaald. Gedurende één jaar kwam elke volwassen patiënt die de huisarts raadpleegde voor stress, urgency of gemengde UI, alsmede patiënten die reeds eerder gediagnosticeerd waren met deze vormen van UI, voor de studie in aanmerking. De geïncludeerde patiënten werden gedurende 12 maanden gevolgd.

Hoofdstuk 5 rapporteert over de effectiviteit van de interventie door gespecialiseerd verpleegkundigen in de PromoCon trial na drie en 12 maanden voor wat betreft de ernst van de UI, zoals gemeten met de ICIQ-UI SF, een gecombineerde uitkomstmaat voor frequentie, hoeveelheid en de impact van UI op de kwaliteit van leven. De data-analyse werd uitgevoerd volgens het intention-to-treat principe. 109 Huisartsen in 65 praktijken namen deel aan de studie en 384 patiënten voldeden aan de inclusiecriteria. Hiervan werden er 186 willekeurig toegewezen aan de interventie en 198 aan care-as-usual. Het merendeel van hen was vrouw (93\%), met een gemiddelde leeftijd van 65 jaar, en de groepen waren vergelijkbaar. Van de 186 patiënten die toegewezen waren aan de interventie door de gespecialiseerd verpleegkundigen, werden er $137(73,6 \%)$ behandeld volgens protocol (zeven tot negen consulten). In gevallen waar er minder consulten werden gegeven dan voorgeschreven door het protocol, werd dit veroorzaakt door gezondheidsproblemen $(n=14)$ of gebrek aan motivatie of een te grote belasting $(n=15)$. Hoewel er geen verschil tussen de groepen werd gevonden in de lineaire trend over één jaar, waren de patienten binnen de groepen aanzienlijk verbeterd voor wat betreft de ernst van en de impact op Ul-specifieke gezondheidsgerelateerde kwaliteit van leven na drie maanden, en voor wat betreft de lineaire trend na één jaar (beide eindpunten $p<0,001$ ). Echter, na correctie voor effectmodificatoren (type UI, body mass index) vonden we significante verschillen tussen de groepen ten voordele van de interventiegroep na drie maanden $(p=0,04)$. Na een jaar werden er geen verschillen gevonden in de lineaire trend $(p=0,15)$. Echter, die patiënten in de interventiegroep die geen angst/depressie hadden gerapporteerd bij aanvang van de trial, verbeterden aanzienlijk meer dan de patiënten in de care-as-usual-groep $(p=0,03)$. Wij concludeerden dat het inzetten van gespecialiseerd verpleegkundigen bij de zorg voor UI patienten als aanvulling op de gebruikelijke zorg door huisartsen, na correctie voor de effectmodificatoren, de ernst en de impact van de UI kan doen verminderen. Dit 
geldt ook in specifieke omstandigheden, zoals angst/depressie. Aangezien UI een chronische dynamische aandoening is, die vaak gecompliceerd wordt door comorbiditeiten, lijkt het positieve effect van de interventie door gespecialiseerd verpleegkundigen op de korte termijn te pleiten voor herhaalde interventies door getrainde professionals in de gezondheidszorg om UI te monitoren en daarmee langetermijn effecten te bereiken. Nader onderzoek zou zich moeten richten op het afstemmen van de interventie op die patiëntkenmerken die prognostische en voorspellende waarde hebben voor UI en die beïnvloedbaar zijn (depressie, overgewicht).

In Hoofdstuk 6 worden de resultaten gepresenteerd van de economische evaluatie vanuit maatschappelijk perspectief, die tegelijk met de RCT is uitgevoerd, ter vaststelling van de kosten-effectiviteit over een periode van 12 maanden van de inzet van in UI gespecialiseerde verpleegkundigen vergeleken met care-as-usual in de eerste lijn. Als uitkomstmaten zijn het voor kwaliteit van leven gecorrigeerde levensjaar (QALY) gebruikt en een urine incontinentie specifieke maat. Gebruikmakend van de EuroQol-5D zijn twee verschillende QALYs berekend: een QALY gebaseerd op de waardering van gezondheid door de algemene populatie ( $Q A L Y_{\text {societal }}$ ) en de QA$L Y_{\text {patient }}$, gebaseerd op de waardering van gezondheid door de patiënt. Daarnaast is de ernst van urine incontinentie somscore van de ICIQ-UI SF gebruikt om een voor de ernst van incontinentie gecorrigeerd levensjaar (ISLY) te bepalen. Kosten binnen de gezondheidszorg, kosten van patiënten en familie, en productieverliezen werden vastgesteld. Kostprijzen waren gebaseerd op Nederlandse standaardprijzen en op eigen kostprijsonderzoek. De gegevens werden verzameld met behulp van driemaandelijkse vragenlijsten. Incrementele kosten-effectiviteitsratio's (ICERS) werden berekend door het verschil in de groepen in kosten te delen door het verschil gemeten op de verschillende uitkomstmaten. Indien de incrementele kosten/ QALY lager zijn dan de drempelwaarde van $€ 40.000$, wordt in Nederland een interventie over het algemeen kosten-effectief gevonden. De mate van onzekerheid werd vastgesteld met behulp van bootstrapsimulatie. Daarnaast werd de bovengrens van de waarde van verder onderzoek om de onzekerheid te reduceren berekend (de zogenaamde verwachte waarde van perfecte informatie). Wij vonden dat de inzet van gespecialiseerd verpleegkundigen, vergeleken met care-as-usual, $€ 16.742$ per gewonnen $\mathrm{QALY}_{\text {societal }}$ kost. Dus ruim beneden de drempelwaarde. Echter, de onzekerheid voor besluitvorming was aanzienlijk. De kans dat de interventie kosteneffectief is is slechts $58 \%$. De verwachte waarde van perfecte informatie is dan ook substantieel: $€ 78$ miljoen. Zowel $Q A L Y_{\text {patient }}$ als ISLY gaf een iets gunstiger kosteneffectiviteit met minder onzekerheid. De ICERS waren aanzienlijk lager wanneer de kosten van de interventie werden gebaseerd op praktijkverpleegkundigen in plaats van gespecialiseerd verpleegkundigen. De subgroepanalyse liet zien dat voor UI patiënten die bij aanvang van de studie geen angst/depressie meldden, de kans dat 
de interventie kosteneffectief is duidelijk hoger is 69\%). Op basis van onze kosteneffectiviteitsresultaten en gezien het feit dat de implementatiekosten waarschijnlijk gering zijn, bevelen wij aan de interventie met gespecialiseerd verpleegkundigen in de eerste lijn in te voeren, onder gelijktijdig uitvoeren van verder onderzoek om onzekerheid te reduceren. Met behulp van zorgvuldige monitoring van de resultaten van de interventie, met adequate registratie van dagelijkse patiëntgegevens, kan een langere follow-up worden gerealiseerd. Dit levert in de toekomst meer gegevens en informatie op voor de besluitvorming ten aanzien van vergoedingen voor Ul zorg.

Naast het onderzoek naar de effectiviteit en de kosten-effectiviteit van het inzetten van gespecialiseerd verpleegkundigen, probeerden wij ook te achterhalen waardoor het effect tot stand komt, met name omdat wij problemen voorzagen met de implementatie van onze interventie vanwege de hervorming van het Nederlandse zorgverzekeringsstelsel in 2006.

Hoewel taakherschikking van huisartsen naar verpleegkundigen steeds gebruikelijker wordt, is niet bekend hoe de verpleegkundigen hun rol ervaren en wat de consumenten (dwz patiënten en huisartsen) van de aanvullende zorg door dit nieuwe type zorgverlener vinden en hoe zij deze zorg ervaren. Dit leidde tot onze derde doelstelling, namelijk het onderzoeken van de haalbaarheid van de interventie. Hiervoor hebben we een kwalitatief focusgroep onderzoek (hoofdstuk 7) uitgevoerd met de gespecialiseerd verpleegkundigen die de interventie hadden uitgevoerd, om te onderzoeken hoe zij hun taak op het gebied van de incontinentiezorg hebben ervaren en op welke manier zij de interventie vorm hebben kunnen geven. Daarnaast is een vragenlijst per post verzonden naar de deelnemende UI patiënten om hun tevredenheid te beoordelen in relatie tot relevante thema's volgens de richtlijnen voor de zorg voor UI patiënten (hoofdstuk 8). Tot slot hebben we na afloop van de studie, steekproefsgewijs, een deel van de deelnemende huisartsen geïnterviewd in een 10 minuten durend gestructureerd telefonisch interview, om ook hun ervaringen met en hun attitude ten opzichte van de interventie door gespecialiseerd verpleegkundigen te onderzoeken, alsmede hun visie op de waarde van deze nieuwe vorm van zorg (hoofdstuk 8). Deze studies waren bedoeld om de potentiële effecten te kunnen verklaren en de belemmerende en bevorderende factoren voor een grootschaliger implementatie te identificeren.

In Hoofdstuk 7 worden de ervaringen en attitudes van de zes gespecialiseerd verpleegkundigen die de interventie hebben uitgevoerd beschreven. In een kwalitatief focusgroeponderzoek onderzochten we hun ervaringen met en hun attitudes ten opzichte van hun rol in de zorg voor patiënten met UI, met als doel het identificeren van bevorderende en belemmerende factoren voor een bredere implementatie. Het bleek dat de gespecialiseerd verpleegkundigen zich competent voelden voor het 
geven van advies en informatie en het bieden van mogelijke oplossingen, alsmede het monitoren en begeleiden van het zorgproces voor mensen met UI. Zij voelden zich gewaardeerd door de patiënten en hadden het gevoel dat ze meerwaarde hadden geboden ten opzichte van de gebruikelijke zorg door huisartsen. De verpleegkundigen vonden dat de huisartsen soms weinig interesse toonden voor UI. Belangrijke voorwaarden voor een effectieve zorg door de gespecialiseerd verpleegkundigen waren persoonlijk contact met de huisartsen en het kunnen beschikken over voldoende tijd, voldoende uitrusting en financiële middelen. De gespecialiseerd verpleegkundigen stelden nascholing en feedback tijdens de dagelijkse zorg voor UI patiënten erg op prijs. We concludeerden dat goed opgeleide gespecialiseerd verpleegkundigen zich competent voelen voor en tevreden zijn over de ondersteuning die zij geven aan huisartsen bij de zorg voor patiënten met ongewild urineverlies, en dat zij zich zeer gewaardeerd voelen door zowel patiënten als huisartsen. Bovendien ondersteunen deze bevindingen de toenemende inzet van gespecialiseerd verpleegkundigen in de Nederlandse huisartsenzorg en dragen ze bij aan de kennis over de haalbaarheid van dit type zorgaanbod door gespecialiseerd verpleegkundigen, zowel vanuit nationaal als internationaal perspectief.

Hoofdstuk 8 beschrijft de tevredenheid onder de consumenten, te weten de patiënten en de huisartsen, over de door dit nieuwe type zorgverlener geleverde zorg. De tevredenheid onder patiënten over de geleverde zorg door hetzij gespecialiseerd verpleegkundigen hetzij huisartsen werd gemeten met een door de patiënten ingevulde vragenlijst. De mening van de huisartsen over het inzetten van de gespecialiseerd verpleegkundigen werd bepaald door middel van een gestructureerd telefonisch interview. De patiënttevredenheidsscore over de door gespecialiseerd verpleegkundigen verleende zorg bedroeg 8,4 (schaal 1-10), versus 6,4 voor care-asusual door de huisarts. Meer dan $85 \%$ van de patiënten zou de zorg door gespecialiseerd verpleegkundigen aanbevelen aan hun beste vrienden, en $77 \%$ van de huisartsen vond de rol van de gespecialiseerd verpleegkundige nuttig en beoordeelde deze met een gemiddelde score van 7,2 . We concludeerden dat herschikking van taken van huisarts naar gespecialiseerd verpleegkundigen bij de zorg voor UI gunstig was ontvangen door zowel patiënten als huisartsen. Kleine veranderingen, zoals het geven van additionele specifieke informatie over UI en het schenken van meer aandacht aan UI (een aandoening die voorheen te weinig aandacht kreeg), vormen een eenvoudig instrument om patiënten te stimuleren hun gedrag in de juiste richting te veranderen.

Tot slot wordt in hoofdstuk 9, de algemene discussie, een kort overzicht gegeven van de achtergrond en onderzoeksdoelstellingen van de verschillende studies in dit proefschrift. De belangrijkste bevindingen worden samengevat en uitgelegd, en gecombineerd in een algemene conclusie. Methodologisch sterke en zwakke punten 
van de algemene aanpak van ons onderzoek worden besproken, alsmede de verschillende kwantitatieve en kwalitatieve methoden die we hebben gebruikt om het construct van de UI zorg en de interpretatie van de resultaten te evalueren. Ook worden de implicaties en aanbevelingen voor verder onderzoek en de praktijk van UI gezondheidszorg gepresenteerd.

Onze algemene conclusie, op basis van de resultaten van de verschillende studies in dit project en gegevens uit lopende studies en de literatuur, is dat het inzetten van deze gespecialiseerde UI verpleegkundigen als waardevol kan worden beschouwd. Om precies te zijn, gezien de gebleken haalbaarheid van de interventie en de resultaten voor wat betreft de kosten-effectiviteit, en het feit dat de implementatiekosten waarschijnlijk laag zijn, bevelen wij aan de interventie door de gespecialiseerd verpleegkundige in de eerstelijnszorg op te nemen, onder gelijktijdig uitvoeren van observationeel onderzoek. Deze implementatie kan alleen succesvol zijn wanneer aan een aantal voorwaarden is voldaan. Het invoeren van interventies door gespecialiseerd verpleegkundigen in de dagelijkse praktijk biedt de gelegenheid om de stappen 'monitoring en herbeoordeling' van de 'TAIL' aanpak onder een representatieve steekproef uit te voeren, met gebruikmaking van een uniform monitoringsysteem voor alle concurrerende zorgconstructies voor UI. in het hoofdstuk worden ook methodologische aanbevelingen voor dergelijke observationele studies gepresenteerd.

Een dergelijke observationele studie, waarin gespecialiseerd verpleegkundigen de huisarts ondersteunen en taken overnemen, alsook een centrale rol spelen bij het tijdig identificeren van UI, het monitoren van de effecten van alle gestarte UI behandelingen en het delen van ervaringen, met name met eerstelijns bekkenfysiotherapeuten en continentieverpleegkundigen, zou als titel kunnen krijgen: 'Who really cares? Aspects of primairy care for UI.' Dergelijk onderzoek kan ertoe bijdragen dat het inzetten van goed opgeleide gespecialiseerd verpleegkundigen in de eerstelijns zorg voor UI patiënten kan leiden tot een effectieve en efficiënte zorg en een lagere ziektelast door UI. 
Co-authors \& affiliations 

(Bary) L.C.M. Berghmans, PhD, MSc, $\mathrm{PPT}^{\circledR}$

Pelvic care Center Maastricht (PcCM),

Maastricht University Medical Centre ${ }^{+}$, Maastricht

(Manuela) M.A. Joore, PhD

Dept. of Clinical Epidemiology and Medical Technology Assessment and

Health Services Research*

Maastricht University Medical Centre ${ }^{+}$, Maastricht

(Ank) A. De Jonge, PhD, Midwife/senior researcher

Midwifery Science/ EMGO Institute for Health and Care research

VU university medical center, Amsterdam

(Toine) A.L.M. Lagro-Janssen, Professor, PhD, MD

Women's Studies in Medicine

Dept. of Primary and Community Care

Radboud University Nijmegen Medical Centre, Nijmegen

(Fred) F.H.M. Nieman, PhD

Dept. of Clinical Epidemiology and Medical Technology Assessment*

Maastricht University Medical Centre ${ }^{+}$, Maastricht

(Hans) J.L. Severens, Prof, PhD

Former: Dept. of Health Organisation, Policy, and Economics and

Clinical Epidemiology and Medical Technology Assessment*

Maastricht University Medical Centre ${ }^{+}$, The Netherlands

Present: Institute of Health Policy and Management

Erasmus University Rotterdam, Rotterdam

(Pieter) P.L. Venema, PhD, urologist

Bekkenbodem Centrum

HAGA Hospital, The Hague

(Ron) R.A.G. Winkens, PhD, MD

Dept. of Integrated Healthcare and General Practice*

Maastricht University Medical Centre ${ }^{+}$, Maastricht

*Departements involved in Maastricht University Medical Centre ${ }^{+} /$

CAPHRI, School for Public Health and Primary Care of the Faculty of Health, Medicine and Life Sciences, Maastricht, The Netherlands. 

Dankwoord 

Als je al 34 jaar werkzaam bent en niemand meer durft te vragen wanneer die studie of zoiets toch eindelijk eens klaar is, is het moment nu daar: het 'proefschrift' is klaar. Maar hoe leg je zoiets uit? Daarom een terugblik over een periode met een hoge P-factor.

Allereerst wil ik alle (huis)artsen, hun assistenten, verpleegkundigen, apothekers, fysiotherapeuten en instanties bedanken die meegeholpen hebben om patiënten met ongewild urineverlies te kunnen benaderen voor het vragenlijstonderzoek en voor de trial of anderszins een bijdrage hebben geleverd aan de onderzoeken. De patiënten dank ik voor hun deelname aan de survey en de trial. Al deze Personen hebben ons zoveel meer inzicht gegeven in de huidige incontinentiezorg en hebben het mogelijk gemaakt om nu te kunnen vaststellen dat ondersteuning van de huisarts door in incontinentie gespecialiseerd verpleegkundigen een waardevol plan is om de kwaliteit van de zorg voor incontinentie te verbeteren.

Dit Plan, dat leidde tot mijn Proefschrift, heb ik alleen dankzij de ideeën en steun van velen ten uitvoer kunnen brengen. Mijn dagelijks begeleidingsteam en Promotiecommissie, jullie bedank ik van harte voor de veelvuldige fikse discussies rond alle belangrijke beslismomenten gedurende de onderzoeken en het schrijven van de papers, jullie kritische houding, wijze lessen, raad en vooral ook de betrokkenheid en support in dit niet altijd even makkelijke multicenter Project.

Dr. M. A. Joore, beste Manuela, de max is 4 , dat is dus vreselijk Pech bij een onderzoek met zoveel disciplines, daarom verdien jij hier nu de eerste Plaats. Heel veel dank voor je coaching bij mijn reis door de wondere wereld van HTA, Excel, de Prettige uurtjes klussen en bootstrappen achter de PC en je rake en ideeënrijke inbreng gedurende het hele project. En...wat klinkt dat lief he, ISLY.

Dr. L.C.M. Berghmans, beste Bary, medeprojectleider, copromotor en collega bekkenfysiotherapeut/bewegingswetenschapper/epidemioloog, jij bent wel de grote initiator, vanuit het Pelvic care Center Maastricht, van de projecten waarvan dit proefschrift verslag van doet. Zonder jouw ideeën, enorme inzet, gedrevenheid en voortvarendheid waarbij ieder nee een uitgesteld ja is, was de PromoCon trial, na honorering door ZonMW, nooit gestart, en ik ook niet met dit Promotietraject en motherfit. Dank voor je vertrouwen. Voor jou is mijn één na laatste stelling. Als de aanbeveling van dit proefschrift werkelijk ten uitvoer wordt gebracht komt er uiteindelijk toch een antwoord op onze allereerst geformuleerde onderzoeksvraag namelijk de interventie door de bekkenfysiotherapeut vergelijken met de gebruikelijke UI zorg al dan niet met een gespecialiseerd verpleegkundige.

Dr. R.A.G. Winkens, beste Ron, projectleider en copromotor vanuit de Transmurale Zorg van het azM, ons contact en mijn eerste kennismaking met jou en de mores rond onderzoek in de huisartsenwereld dateert van de pogingen om de survey onder huisartsen via het CEL uit te zetten. Met jouw bijzondere timing (dit werkje is 
mooi op tijd klaargekomen), je Politiek laveren, bijvoorbeeld toen de regionale $\mathrm{CIZ}$ alsnog de indicering voor de AWBZ introk in Maastricht, je zeer verrassende invalshoeken, je 'boodschappen', je fraaie formuleringen en de kunst van het weglaten, heb je me wegwijs gemaakt in de wereld van onderzoek en wetenschapsverslaglegging. Ik heb veel van je geleerd.

Prof. Dr. A.L.M. Lagro-Janssen, lieve Toine, tweede Promotor, jouw enorme Persoonlijke betrokkenheid bij het onderwerp van dit proefschrift en het hele project, en vooral ook de werving in en rond Nijmegen, jouw levenskracht, jouw daadkracht op alle niveau's, jouw snelheid van handelen en de moeite die je steeds nam om af te reizen naar het verre zuiden zijn werkelijk verbijsterend. leder gesprek met jou en ieder door jou becommentarieerd manuscript was een bron van inspiratie, waar ik steeds mee verder kon. Ik heb genoten van de opleidingsperiode en de terugkombijeenkomsten met de verpleegkundigen die wij samen hebben begeleid en de studiemiddagen 'Vrouwenstudies Medische Wetenschappen', wat een diversiteit aan inspirerende onderwerpen en collega's! Heel veel dank voor het gevoel van vertrouwen dat je me steeds hebt gegeven tussen de corrigerende boodschappen en wijze lessen door.

Dr. F. Nieman, beste Fred, het was een langdurig project met lange namen en in een zekere fase met veel sterke koffie en figuurlijke ijsemmers om het hoofd koel te houden. Daarom nu een kort en bondig dank voor al je statistische hulp, ondersteuning en niet te vergeten je kritische beschouwingen. Je leerde me: "mevrouw Albers, u moet nooit iets weggooien" en over de eyeball methode.

Professor Dr. J. L. Severens, beste Hans, eerste promotor, weer een Maastrichtse klus geklaard, ondanks je vertrek naar Rotterdam. Jouw scherpzinnige blik, je scherpe analyses, je knuppels in het hoenderhok, je Pragmatische ideeën, je stimulerende en toegankelijke Persoonlijkheid en je brede belangstelling en doortastende rol waren een genot en onmisbaar zeker in de laatste fase. Voor jou de laatste stelling.

Dank aan de leden van de beoordelingscommissie Prof. Dr. Ph.E.V.A. Van Kerrebroeck, voorzitter, Dr. M.G.W. Dijkgraaf, Prof.dr. J.P.H. Hamers, Prof. dr. J.F.M. Metsemakers en Prof. Dr. R. Nijhuis-Van der Sanden voor uw kritische aandacht voor mijn proefschrift en de bereidheid het te beoordelen op zijn kwaliteit.

Veel dank aan al degenen die op de een of andere manier onmisbare assistentie hebben verleend bij de onderzoeksopzet, uitvoering, dataverzameling en datainvoer.

Wendy Engering, lieve Wendy, jij was een onmisbare onderzoeksassistente en de stuwende kracht van het eerste uur bij de uitvoering van de surveys, de opzet van de trial in Maastricht en Helmond, het aansturen van de assistentie in de andere regio's, de huisartsen- en patiëntenrekrutering, de dataverzameling en invoer. Petje af. Met jou kun je zaken doen, je weet van aanpakken, zelfstandig opereren, 
afhandelen en altijd een opgeruimd bureau, je bent recht door zee en op de man af. We hebben samen veel geleerd, veel plezier gehad en hadden een goede match, veel dank en dat houden we zo!

Margriet Straver, lieve Margriet, je bent een schat en bijzonder aangenaam mens! Dank voor de patiënten werving en de dataverzameling in Nijmegen, samen hebben we een hele klus geklaard. Dank voor je hartelijke zorg en aandacht voor de bijeenkomsten met de verpleegkundigen, je betrokkenheid bij en het uittypen van het focus groep interview, helemaal jouw ding, en het interviewen van de huisartsen.

Liesbeth Hoff-van Hoef, lieve Liesbeth, ik ben je allereerst heel veel dank verschuldigd voor het overnemen van assistentie, na het vertrek van Wendy in een bijzonder hectische periode. Behalve het includeren van patiënten hield je je bezig met data-invoer en de huisartseninterviews. Je deed dat fantastisch. Maar daarnaast ben je gewoon een lekker betrokken, geïnteresseerd en gePassioneerd mens en altijd bereid tot hand en spandiensten.

Janny de Jonge, onderzoeksassistente in Den Haag vanuit het Poliklinisch Continentie Centrum, veel dank voor jouw Haagse werving, een vak apart!

Secretariaat QUARTZ, transmuraal centrum van de regio Helmond, Elkerliek ziekenhuis Bea Heesakkers, dank voor de hulp bij de werving van huisartsen.

Marion Bosch, dank voor je multi-inzetbaarheid, je deelname aan de opleiding, en je hand en spandiensten bij de werving in Helmond en omstreken.

Guy Schulpen, en vooral Joyce Janssen, zonder jullie hulp had ik nooit zo'n mooie handige Access database kunnen maken die uiteindelijk foutloos functioneerde. Joyce, wat fijn dat ik altijd met mijn vragen over Access, data, PC's bij jou terecht kon en nu ook nog met mijn adreslijsten!

Huub Verheijden en zoon, dank voor de data-invoer van de surveys en het tackelen van de eerste Access bugs.

Nicole Castro, lieve Nicole, wat heb je hard, nauwkeurig, Perfect en met een bewonderenswaardig engelengeduld data uitgezocht en ingevoerd. Je weet nu alles van maten, soorten en prijzen van inco materiaal, maar hebt vooral geleerd wat je wel en niet wilt in het werkende leven!

Diana Kerner, Greet Willemsen, dank voor het versturen van nieuwsbrieven, vergaderingen plannen en hand en spandiensten.

Een bijzonder woord van dank heb ik voor de verpleegkundigen die met zoveel inzet en hartverwarmend enthousiasme de interventie, na een pittige opleiding met toets, hebben uitgevoerd en van hun werkgevers de gelegenheid kregen geboden om aan dit onderzoeksproject deel te nemen. De terugkombijeenkomsten met jullie waren inspirerend. Het was een voorrecht om bij jullie in de keuken te mogen kijken die jullie stuk voor stuk vol trots hebben laten zien. Bij jullie staat de patiënt 
echt centraal. Dank voor jullie deelname aan de focusgroep waarin jullie ervaringen in je nieuwe rol zo mooi naar voren zijn gekomen en deze met ons hebben gedeeld.

Alie Offringa, veel dank voor het opzetten van je werkplek, je profileren naar huisartsen en het begeleiden van alle patiënten in Maastricht. Als verpleegkundige in de thuiszorg was je al aardig door de wol geverfd. Met jou heb ik veel prettige uurtjes doorgebracht om vorm te geven aan deze nieuwe tak van sport, waar jij vanuit je positie bij Groene Kruis Domicura (dank aan Pierre Dols, Nicole Quadackers, Lisette Ars) en bij het Pelvic care Center kon deelnemen.

Marjo van Dijk, Brenda Groos, Gerdien Verhoeven jullie waren geweldige verpleegkundigen vanuit de Zorg Groep Zuid-Gelderland (dank aan Ricky Hendriks, Hans Wolters) en verantwoordelijk voor het opzetten van je werkplek in overleg met de huisartsen en het leveren van de patiëntenzorg in Nijmegen en omstreken. Het is mooi om te horen hoeveel meer aandacht ieder van jullie binnen je werk nu besteden aan de juiste begeleiding van patiënten met ongewenst urineverlies. Gerdien, jouw rol bij de opleiding van de verpleegkundigen in het kader van de implementatie van ImPromoCon was wel even spannend maar helemaal geslaagd!

Jeanne Verhees, jij klaarde de interventieklus maar mooi in je eentje in de ruime regio Helmond vanuit De Zorgboog (dank aan Manon van de Heuvel) en Quartz van het Elkerliek Ziekenhuis, (dank aan Hans Vlek) waar al een basis voor de zorg voor incontinentie patiënten was gelegd door Henk Seelen.

Petra van Sinten, voor jou als zeer ervaren continentieverpleegkundige voor Bosman bv en het Poliklinisch Continentie Centrum van het Leyenburg ziekenhuis (thans Haga ziekenhuis) in Den Haag was dit toch weer een ander domein om je mee bezig te houden. Dit heb je 'toch maar mooi in je koffertje meegenomen'. Mijn dagje mee op pad in de Schilderswijk was een ervaring! Dank voor de steun van AZIVO (Fred vd Heuvel, Paul den Daas, Suzanne de Vries, later Marieke Bros), Stichting Haagse Gezondheidscentra, Anne van Popta).

De deelname van Den Haag is geheel en al geïnitieerd en geïnspireerd door Dr. Pieter Venema van het PcCM. Beste Pieter, dank voor al je hartelijke hulp in het Haagse bij de voorbereiding, opzet en uitvoering van de interventie. De praktijk bleek weerbarstig en geeft stof tot nadenken. Ook wil ik je als coauteur bedanken voor je zorgvuldig meelezen en je heldere commentaar.

Daarnaast bedank ik graag al degenen die meegeholpen hebben aan het geven van de opleiding aan de verpleegkundigen en die hen de mogelijkheid hebben geboden tot het lopen van stage om kennis te maken met het brede domein van incontinentie (bekkenfysiotherapeuten, incontinentieverpleegkundigen en afdelingen urodynamica).

Dr. Ank De Jonge, verloskundige en onderzoeker, heel veel dank voor de gezellige en fijne samenwerking, jouw spontane en deskundige hulp bij de opzet, het modereren en analyseren van de focus groep, mijn 1e stappen op het gebied van kwalitatief 
onderzoek, en het tot stand komen van het artikel, ook al was je druk met de laatste loodjes van jouw eigen promotie.

Jennifer Dennison en Jan Klerkx dank ik van harte voor het engels redigeerwerk waardoor mijn 'europe-english' aardig is bijgeschaafd.

Ruud Leliveld dank voor de zorgvuldige lay-out en het verzorgen van de productie van dit proefschrift.

Ik heb dit onderzoek kunnen uitvoeren binnen de Transmurale Zorg van het azM, sinds kort Patient en Zorg geheten, een afdeling in beweging.

Hans Fiolet, ons contact was steeds kort, maar van invloed. Jou bedank ik voor de mogelijkheden die je me hebt gegeven, nooit klopte ik tevergeefs bij je aan.

(ex)Onderzoekers en medewerkers TZ in het MECC niveau 3, 1 of elders, die ik nog niet eerder in dit dankwoord noemde, Angelique Dierick, Caro van Uden, Nicole Maraite, Trudy vd Weijden, George Beusmans, Jody Martens, Alied van der Aa, Inge Duimel, Bert Vrijhoef, Ireen Proot, Josiane Boyne, Yvonne van Eijk, Mariëlle Kroese (het eind is in zicht), Monique Bessems, Juliane Strassel, Manon Fens, Suus Benjaminsen, Ron Heijnen, Daisy de Bruijn, Carla Stessen, dank dat jullie er op enig moment waren, ook al was ik er niet zo vaak. Dank voor alle discussies, tips over onderzoek, maar vooral ook de gezellige Praat en broodnodige afleiding. Helene Voogdt, met jou heb ik het langst een kamer gedeeld tot jouw eigen promotie in het voorjaar, altijd tijd voor een goed gesprek, het was me waarlijk een bijzonder groot genoegen! Greetz!

Dr. Arianne Verhagen, beste Arianne, Prof. Dr. Rob de Bie, beste Rob, en Dr. Ir. Martien van Dongen, beste Martien, dankzij jullie heb ik jaren terug Plezier en Passie voor onderzoek ontwikkeld. Veel dank daarvoor!

Leefbaar Bunde, Chris, Max en Frits, 'maten van het onderzoek en wetenschap', wat heerlijk om met regelmaat uitbundig verbaal, sportief en culinair bij te tanken!

Nu naar de Praktijkbasis: Ellen Hawinkels, lieve Ellen, ik vind het een eer dat jij vandaag naast mij staat als paranimf. Het borrelde en broeide van ideeën van dag één bij fysiotherapie Roex (veel dank aan Hans Roex, Miriam van Neer) en dat doet het tot op de dag van vandaag bij Basaal. Met een fijne neus voor ontwikkelingen en innovatie reizen we regelmatig samen de wereld rond, gebeurt en kan er veel. Dank voor al je enthousiasme en stimulans om kansen te grijpen. Ik ga graag nog een tijdje door met jou en onze lieve Basaal collega's Harma Hoekstra, Marguerite Hoogland, Jelka van Manen, Martine Relou, Wanda de Goeij en Leo Vervoort!

Een onmisbare basis en van onschatbare waarde voor het vak en voor mijzelf als mens zijn nu, of op enig moment geweest, mijn geweldige inspirerende collega (bekken)fysiotherapeuten van het eerste uur: Alexandra Vermandel, Marijke Slieker, 
Joke Groot, Fetske Hogen Esch, Marijke van Kampen, Lilian Smeets, Ciel Aghina, Ineke de Gee, Jenneke Kalkdijk en ieder ander die ik hier nu ongetwijfeld vergeet.

Tot slot, meestal ga ik met Plezier naar het werk maar met zeker zoveel plezier naar huis.

Dank aan mijn femmelie, familie en bekenden, die bijna niet meer durfden te vragen of niet meer wisten waar ik zo druk mee was, voor hun belangstelling op enig moment. Een paar mensen wil ik speciaal bedanken.

Mijn lieve vriendinnen, Ans, Miets en Paulie, jullie zijn mijn vaste baken helemaal los van onderzoek of werk samen met jullie achterban.

Akkie, bambola, ama, sista mi, un'amica stretta en paranimf, wat hebben we al veel samen gedeeld tijdens onze vele tochten te voet, op de schaats, het ijzeren ros en in het leven, en daarna we bellen nog wel he! Ik vind het een eer dat jij vandaag samen met Ellen naast mij staat! Verstandige keuze he... ...van Ghana tot Dolomiti lets keep in mind: 'Life is wonderful'.

Annelies en Marleen, altijd stonden jullie klaar onder het genot van een goed glas en met een goed bed, zeker in de opzetfase van mijn onderzoek, fantastisch!

Lia, lieve zus, het waren me turbulente jaren, je bent van onschatbare waarde in mijn levensPad.

Floor, Okke, Corine en Xander, Anne, wat ben ik apetrots op jullie al vonden jullie het af en toe wel erg gestoord dat ik op mijn leeftijd nog zo vaak achter de PC moest zitten in de vrije uren! Jullie pakken dat ongetwijfeld anders aan! Okke, dank voor je Access hulp al was het wel wat chantage om niet aan de drugs te raken. Floor, dank voor het koerieren en datacleanen in ruil voor wat naaiwerk. Xander, aan jou dank ik de Prachtige omslag van dit proefschrift, chapeau!

Liefste $E d$, dank je wel dat jij er bent. Un bacio e invecchia insieme a me, il meglio deve ancora venire. 


\section{Curriculum Vitae}




\section{Curriculum Vitae}

Pytha Albers-Heitner werd geboren op 21 september 1953 in Eindhoven. Na het behalen van het gymnasium diploma aan het van der Putt lyceum in Eindhoven en de studie fysiotherapie in 's Hertogenbosch vertrok zij voor $2 \frac{1}{2}$ jaar naar Oslo, Noorwegen, waar zij zich naast haar werk als fysiotherapeut specialiseerde in de manuele therapie bij Olaf Evjenth en Freddy Kaltenborn. In 1980 werkte zij in het Kaiser Foundation Rehabilitation Center in Vallejo, California, USA, als junior staflid en specialiseerde zich in de behandeling van neurologische aandoeningen met Proprioceptive Neuromuscular Facilitation techniques (PNF). Eenmaal terug in Nederland vond zij een ideale werkplek bij Revalidatie Centrum Blixembosch, Eindhoven. Aangezien part-time werken als moeder van 3 kinderen niet gebruikelijk was in de jaren tachtig stapte zij over naar particuliere fysiotherapie praktijk Der Kinderen in Veldhoven. In die tijd volgde zij diverse specialisaties in zwangerschapsbegeleiding, obstetrie en urogynaecologie waarmee de basis werd gelegd voor haar interesse voor het onderwerp van dit proefschrift. Vanaf 1993 tot heden werkt zij als bekkenfysiotherapeut bij Basaal, expertisecentrum voor bekkenfysiotherapie en seksuologie, voorheen Fysiotherapie Roex en Hawinkels, in Waalre. Hier ziet zij dagelijks patiënten met bekkenbodemklachten zoals ongewild urineverlies. Vanwege het enorme gebrek aan wetenschappelijk bewijs op het gebied van de conservatieve behandeling van bekkenbodemklachten, waaronder urine incontinentie, startte zij in 1996 de deeltijdstudie gezondheids/bewegingswetenschappen en epidemiologie aan de Universiteit Maastricht. In 2000 studeerde zij cum laude af met een valideringsonderzoek naar palpatie en myofeedback bij vrouwen met symptomen van stress urine-incontinentie onder begeleiding van Dr. Martien van Dongen, Prof. Dr. Rob de Bie en Dr. Marijke Slieker-Ten Hove. Sinds die tijd is zij betrokken bij diverse (onderzoeks)projecten, onderzoeksaanvragen en onderwijsactiviteiten op het gebied van bekken(bodem)dysfuncties. In 2004 hoorde zij bij de eerste lichting geregistreerde bekkenfysiotherapeuten ${ }^{\circledR}$. In 2004 werd zij aangesteld als deeltijd onderzoeker voor het onderzoeksproject dat leidde tot dit proefschrift bij de RVE Transmurale zorg, thans Patient en Zorg, van het Maastricht Universitair Medisch Centrum+. Sinds 2009 is zij betrokken bij het motherfit onderzoeksproject, een multidisciplinair programma ter verbetering van de kwaliteit van de preventieve zorg voor urine incontinentie bij post-partum vrouwen. Dit onderzoek is geinitieerd door het Pelvic care Center Maastricht en CAPHRI, the School for Public Health and Primary Care, FHML. 


\section{Curriculum Vitae}

Pytha Albers-Heitner was born on September 21, 1953 in Eindhoven. After graduating from secondary school at the van der Putt Lyceum in Eindhoven and physiotherapy in 's Hertogenbosch, she left for $2 \frac{1}{2}$ years to Oslo, Norway where she worked as a physiotherapist and specialised in manual therapy with Freddy Kaltenborn and Olaf Evjenth. In 1980 she worked as a junior staff member in the Kaiser Foundation Rehabilitation Center in Vallejo, California, USA, and specialised in the treatment of neurological diseases with Proprioceptive Neuromuscular Facilitation (PNF) techniques. Back in the Netherlands she found an ideal workplace in Blixembosch Rehabilitation Centre, Eindhoven. Since part-time work as a mother of three children was not common she moved in the late eighties to private practice physiotherapy Der Kinderen in Veldhoven. During that period she specialised in pre and postpartum care, obstetrics and urogynaecology which was the basis for her interest in the topic of this thesis. From 1993 until present she works as a pelvic physiotherapist at Basaal, expert centre for pelvic physiotherapy and sexology, formerly Physiotherapy Roex and Hawinkels in Waalre. Here she sees patients with pelvic floor dysfunctions including incontinence in every day practice. Due to the sheer lack of scientific evidence in the conservative treatment of pelvic floor dysfunctions, including urinary incontinence, she started in 1996 the part-time study health/movement sciences and epidemiology at Maastricht University. In 2000, she graduated cum laude with a validation study of palpation and myofeedback in women with symptoms of stress urinary incontinence under the supervision of Martien van Dongen, PhD, Prof. Rob de $\mathrm{Bie}, \mathrm{PhD}$ and Marijke Slieker-Ten Hove, PhD. Since then she is involved in several (research) projects, applications and educational activities in the field of pelvic (floor) dysfunctions. In 2004, she belonged to the first registered Dutch pelvic physiotherapists ${ }^{\circledR}$. In 2004 she was appointed at the Dept. of Integrated Care, Maastricht University Medical Centre+, for the research that led to this thesis. Since 2009 she is involved in the motherfit research project, a multidisciplinary programme to improve the quality of preventive care for urinary incontinence in pre and postpartum women, initiated by the Pelvic Care Centre Maastricht and CAPHRI, the School for Public Health and Primary Care, FHML. 


\section{Publications related to this thesis}

Berghmans Bary, Albers-Heitner Pytha, Winkens Ron. Systematic review betreffende de interventie van de gespecialiseerd verpleegkundige bij patiënten met urineincontinentie. Incourant VNIV. 2005(27):10-17.

Berghmans Bary, Albers-Heitner Pytha, Winkens Ron. Systematic review betreffende de interventie van de gespecialiseerd verpleegkundige bij patiënten met urineincontinentie. NVFB Bulletin. 2006(18):23-26.

Albers-Heitner CP, Berghmans LCM, Joore M, Lagro-Janssen ALM, Severens JL, Nieman FHM, Winkens, RAG. Study protocol. The effects of involving a nurse practitioner in primary care for adult patients with urinary incontinence: the PromoCon study (Promoting Continence). BMC Health Services Research. 2008(8):84.

Albers-Heitner CP, Berghmans LCM, Nieman FHM, Lagro-Janssen ALM, Winkens RAG. Adherence to professional guidelines for patients with urinary incontinence by general practitioners: a cross-sectional study. J Eval Clin Pract. 2008;14(5):807-11.

Albers-Heitner CP, Berghmans LCM, Nieman FHM, Lagro-Janssen ALM, Winkens RAG. How do patients with urinary incontinence perceive care given by their general practitioner? A cross-sectional study. Int J Clin Pract. 2008;62(3):508-15.

Winkens RAG, Albers-Heitner CP, Berghmans LCM, Joore MA, Lagro-Janssen ALM, Severens JL, Nieman FHM. Report for the Netherlands Organisation for Health Research and Development. The effects of involving a nurse practitioner in primary care for adult patients with urinary incontinence: the PromoCon study (Promoting Continence), a multi center pragmatic randomised controlled clinical trial. Trial registration: Current Controlled Trials ISRCTN62722772; PROJECTNUMBER: 945-04-224, the Netherlands Organisation for Health Research and Development. Maastricht, 30-09-2008.

Albers-Heitner CP, Berghmans LCM, Lagro-Janssen ALM, Joore MA, Nieman FHM, Venema PL, Severens JL, Winkens RAG. Effectiveness of involving a nurse specialist for patients with urinary incontinence in primary care: results of a pragmatic multicentre randomised controlled trial [Abstract, poster podium presentation ICS 2009, San Francisco, USA]. Neurourol Urodynam. 2009;28(7):815.

Albers-Heitner CP, Joore MA, Winkens RAG, Lagro-Janssen ALM, Severens JL, Berghmans LCM. Cost-effectiveness of involving nurse specialists for adult patients with UI in primary care: an economic evaluation alongside a pragmatic randomized 
controlled trial based on generic health related quality of life and UI specific outcomes [Abstract, poster podium presentation ICS-IUGA 2010 Toronto, Canada]. Int Urogynecol J. 2010;21(Suppl 1):S240. Scientific program. Neurourol Urodynam. 2010;29(6):805-1255.

Albers-Heitner CP, Lagro-Janssen ALM, Venema PL, Berghmans LCM, Winkens RAG, de Jonge $A$, Joore, MA. Experiences and attitudes of nurse specialists in primary care regarding their role in care for patients with urinary incontinence. Scand J Caring Sci. 2011;25(2):303-10.

Albers-Heitner CP, Lagro-Janssen ALM, Joore MA, Berghmans LCM, Nieman FHM, Venema PL, Severens JL, Winkens RAG. Effectiveness of involving a nurse specialist for patients with urinary incontinence in primary care: results of a pragmatic multicentre randomised controlled trial. Int J Clin Pract. 2011;65(6):705-12.

Albers-Heitner CP, Joore MA, Winkens RAG, Lagro-Janssen ALM, Severens JL, Berghmans LCM. Cost-effectiveness of involving nurse specialists for adult patients with urinary incontinence in primary care compared to care-as-usual: an economic evaluation alongside a pragmatic randomised controlled trial. Neurourol Urodynam. 2011; accepted for publication July 08. 